REPRESENTATION THEORY

An Electronic Journal of the American Mathematical Society

Volume 11, Pages 122-171 (August 29, 2007)

S 1088-4165(07)00320-2

\title{
A CLASS OF PERVERSE SHEAVES ON A PARTIAL FLAG MANIFOLD
}

\author{
G. LUSZTIG
}

\begin{abstract}
We introduce a class of perverse sheaves on a partial flag manifold of a connected reductive group $G$ defined over a finite field which are equivariant for the action of the group of rational points of $G$. The definition of this class is similar to the definition of parabolic character sheaves.
\end{abstract}

\section{INTRODUCTION}

Let $f: X \rightarrow Y$ be a morphism of algebraic varieties over an algebraically closed field $\mathbf{k}$. The theory of perverse sheaves BBD associates to $f$ a collection of invariants, namely the collection of simple perverse sheaves on $Y$ which appear as

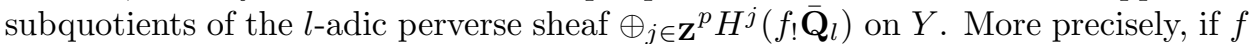
is equivariant for given actions of a finite group $\Gamma$ on $X$ and $Y$, then we denote by $C_{\Gamma}(f)$ the (finite) collection of simple $\Gamma$-equivariant perverse sheaves on $Y$ which appear as subquotients of the $l$-adic $\Gamma$-equivariant perverse sheaf $\oplus_{j \in \mathbf{Z}^{p}} H^{j}\left(f_{!} \overline{\mathbf{Q}}_{l}\right)$ on $Y$. (The perverse sheaves in $C_{\Gamma}(f)$ are not necessarily simple if the $\Gamma$-equivariant structure is disregarded.)

In this paper we try to understand the collection $C_{\Gamma}(f)$ in the case where:

$\mathbf{k}$ is an algebraic closure of a finite field $\mathbf{F}_{q}$,

$G$ is a connected reductive algebraic group defined over $\mathbf{F}_{q}$ with Frobenius map $F: G \rightarrow G$,

$\Gamma$ is the group of $\mathbf{F}_{q}$-rational points of $G$,

$X$ is the variety of all pairs $\left(B^{\prime}, g\right)$ where $B^{\prime}$ is a Borel subgroup of $G$ and $g \in G$ is such that $g^{-1} F(g)$ is in the unipotent radical of $B^{\prime}$,

$Y$ is the variety of parabolic subgroups of $G$ of a fixed type,

$f$ associates to $\left(B^{\prime}, g\right) \in X$ the unique parabolic subgroup in $Y$ that contains $B^{\prime}$.

(The action of $\Gamma$ on $X$ is $g_{0}:\left(B^{\prime}, g\right) \mapsto\left(g_{0} B^{\prime} g_{0}^{-1}, g g_{0}^{-1}\right)$; the action of $\Gamma$ on $Y$ is by conjugation.)

Note that the variety $X$ can be viewed as a family of varieties of the type considered in $[\mathrm{DL}$ indexed by the full flag manifold.

In this paper we define a finite collection $\mathbb{S}(Y)$ of simple $\Gamma$-equivariant perverse sheaves on $Y$ by two methods (see Sections 3 and 4). These methods and the proof of their equivalence are similar to those used in the theory of parabolic character sheaves [L5]. The second method (see Section 4) gives a description of these perverse

Received by the editors December 19, 2006.

2000 Mathematics Subject Classification. Primary 20 G99.

The author was supported in part by the National Science Foundation.

(C)2007 American Mathematical Society Reverts to public domain 28 years from publication 
sheaves in terms of some explicit local systems on some pieces of a finite partition of $Y$. This partition, introduced by the author in 1977, and further studied in [BE], reduces in the case where $Y$ is the full flag manifold to the partition introduced in DL.

In 7.6 we show that $C_{f}(Y) \subset \mathbb{S}(Y)$.

In Section 6 we construct an explicit basis for the space of intertwining operators between certain cohomologically induced representations of $\Gamma$, extending an idea of [L4]. As a bi-product we obtain a disjointness theorem for the objects of $\mathbb{S}(Y)$ which in the special case where $Y=\{G\}$ reduces to the disjointness theorem DL, $6.2,6.3$ ] (but the present proof is quite different from that of [DL).

In Section 7 we study the variety $X$ (see above). In particular we show (using results in Section 6) that $X$ is connected if $G$ is simply connected.

In Section 8 we give a conjecture (based on results in Section 6 and some combinatorial results in Section 5) which should explain in an intrinsic way the "Jordan decomposition" [L1] for irreducible representations of $\Gamma$.

\section{Contents}

1. Preliminaries

2. The variety $\mathcal{Z}^{\mathbf{s}}$ and the local system $\overline{\mathcal{L}}$

3. The class $\mathbb{S}^{\prime}\left(\mathcal{P}_{J}\right)$ of simple objects in $\mathcal{M}_{\Gamma}\left(\mathcal{P}_{J}\right)$

4. The class $\mathbb{S}\left(\mathcal{P}_{J}\right)$ of simple objects in $\mathcal{M}_{\Gamma}\left(\mathcal{P}_{J}\right)$

5. Some computations in the Weyl group

6. A basis for a space of intertwining operators

7. The variety $X$

8. A conjecture

\section{Preliminaries}

1.1. Let $\mathbf{k}$ be an algebraically closed field. In this paper all algebraic varieties are over k. Let $G$ be a connected reductive algebraic group. Let $\mathcal{B}$ be the variety of Borel subgroups of $G$. We fix $B \in \mathcal{B}$ and a maximal torus $T$ of $B$. Let $N(T)$ be the normalizer of $T$ in $G$. Let $W=N(T) / T$. Note that $W$ acts on $T$ by conjugation; we use this action to identify $W$ with a subgroup of $\operatorname{Aut}(T)$. For any $\left(B^{\prime}, B^{\prime \prime}\right) \in \mathcal{B} \times \mathcal{B}$ there is a unique $w \in W$ such that $g B^{\prime} g^{-1}=B, g B^{\prime \prime} g^{-1}=\tilde{w} B \tilde{w}^{-1}$ for some $g \in G$ and some representative $\tilde{w}$ of $w$ in $N(T)$. We then write $\operatorname{pos}\left(B^{\prime}, B^{\prime \prime}\right)=w$. Let $l$ : $W \rightarrow \mathbf{N}$ be the length function: $l(w)=\operatorname{dim}\left(B^{\prime} /\left(B^{\prime} \cap B^{\prime \prime}\right)\right)$ where $\left(B^{\prime}, B^{\prime \prime}\right) \in \mathcal{B} \times \mathcal{B}$, $\operatorname{pos}\left(B^{\prime}, B^{\prime \prime}\right)=w$. Let $\mathbf{I}=\{w \in W ; l(w)=1\}$.

If $H$ is a group acting on a set $X$, we denote by $X^{H}$ the fixed point set of $H$ on $X$.

1.2. Let $\leq$ be the standard partial order on $W$ regarded as a Coxeter group with generators I. If $X$ is a subset of $W$ and $w \in X$ we write $w=\min X$ if $l(w)<l\left(w^{\prime}\right)$ for all $w^{\prime} \in W-\{w\}$. For $J \subset \mathbf{I}$ let $W_{J}$ be the subgroup of $W$ generated by $J$. For $J, J^{\prime} \subset W$ let ${ }^{J} W=\left\{w \in W ; w=\min \left(W_{J} w\right)\right\}, W^{J^{\prime}}=\left\{w \in W ; w=\min \left(w_{J^{\prime}}\right)\right\}$, ${ }^{J} W^{J^{\prime}}={ }^{J} W \cap W^{J^{\prime}}$.

Let $w_{\mathbf{I}}$ be the unique element of maximal length in $W$. 
1.3. Let $\mathcal{P}$ be the variety of parabolic subgroups of $G$. For any $P \in \mathcal{P}$ let $U_{P}$ be the unipotent radical of $P$. We set $U=U_{B}$. For $J \subset \mathbf{I}$ let $P_{J} \in \mathcal{P}$ be the subgroup of $G$ generated by $B$ and by representatives in $N(T)$ of the various elements of $J$. Let $L_{J}$ be the unique Levi subgroup of $P$ that contains $T$. For $s \in \mathbf{I}$ we write $P_{s}$ instead of $P_{\{s\}}$. For $J \subset \mathbf{I}$ let $\mathcal{P}_{J}$ be the $G$-conjugacy class of parabolic subgroups of $G$ that contains $P_{J}$. For $B^{\prime} \in \mathcal{B}$ let $P_{B^{\prime}, J}$ be the unique subgroup in $\mathcal{P}_{J}$ that contains $B^{\prime}$. For $P \in \mathcal{P}_{J}, Q \in \mathcal{P}_{J^{\prime}}$ the element $\operatorname{pos}(P, Q):=\min \{w \in W ; w=$ $\operatorname{pos}\left(B^{\prime}, B^{\prime \prime}\right)$ for some $\left.B^{\prime} \subset P, B^{\prime \prime} \subset Q\right\}$ is well defined and $\operatorname{pos}(P, Q) \in{ }^{J} W^{J^{\prime}}$. We set

(a) $P^{Q}=(P \cap Q) U_{P} \in \mathcal{P}_{J \cap u J^{\prime} u^{-1}}$ where $u=\operatorname{pos}(P, Q)$.

For any $g \in G$ we define $k(g) \in N(T)$ by $g \in U k(g) U$.

1.4. Let $R \subset \operatorname{Hom}\left(T, \mathbf{k}^{*}\right)$ be the set of roots. Let $\check{R} \subset \operatorname{Hom}\left(\mathbf{k}^{*}, T\right)$ be the set of coroots. Let $\check{\alpha} \leftrightarrow \alpha$ be the standard bijection $\check{R} \leftrightarrow R$. For $\alpha \in R$ let $U_{\alpha}$ be the one-dimensional root subgroup (normalized by $T$ ) corresponding to $\alpha$. Let $R^{+}=\left\{a \in R ; U_{\alpha} \subset B\right\}, R^{-}=R-R^{+}$. For $s \in \mathbf{I}$ let $\alpha_{s}$ be the unique root such that $U_{\alpha_{s}} \subset P_{s}$. We write $\check{\alpha}_{s}$ instead of $\left(\alpha_{s}\right)^{\vee}$. The natural action of $W$ on $T$ induces an action of $W$ on $R$.

1.5. For any $s \in \mathbf{I}$ we fix a homomorphism $h_{s}: S L_{2}(\mathbf{k}) \rightarrow G$ such that

$h_{s}\left(\begin{array}{cc}b & 0 \\ 0 & b^{-1}\end{array}\right)=\check{\alpha}_{s}(b)$ for all $b \in \mathbf{k}^{*}$,

$a \mapsto h_{s}\left(\begin{array}{cc}1 & a \\ 0 & 1\end{array}\right)$ is an isomorphism $x_{s}: \mathbf{k} \stackrel{\sim}{\rightarrow} U_{\alpha_{s}}$,

$a \mapsto h_{s}\left(\begin{array}{cc}1 & 0 \\ a & 1\end{array}\right)$ is an isomorphism $y_{s}: \mathbf{k} \stackrel{\sim}{\rightarrow} U_{\alpha_{s}^{-1}}$.

We say that $\left\{B, T, h_{s}(s \in \mathbf{I})\right\}$ is an épinglage of $G$. Let $\dot{s}=h_{s}\left(\begin{array}{cc}0 & 1 \\ -1 & 0\end{array}\right) \in N(T)$. For any $w \in W$ we set $\dot{w}=\dot{s}_{1} \dot{s}_{2} \ldots \dot{s}_{n} \in N(T)$ where $s_{1}, s_{2}, \ldots, s_{n} \in \mathbf{I}$ are chosen so that $w=s_{1} s_{2} \ldots s_{n}, l(w)=n$. (This is independent of the choice.) In particular, $\dot{1}=1$.

For any sequence $\mathbf{w}=\left(w_{1}, w_{2}, \ldots, w_{r}\right)$ in $W$ we set $[\mathbf{w}]=w_{1} w_{2} \ldots w_{r} \in W$ and $[\mathbf{w}]^{\bullet}=\dot{w}_{1} \dot{w}_{2} \ldots \dot{w}_{r} \in N(T)$.

1.6. Equivariant structures. If $X$ is an algebraic variety we write $\mathcal{D}(X)$ for the derived category of bounded constructible $\overline{\mathbf{Q}}_{l}$-sheaves on $X$. If $K \in \mathcal{D}(X)$, let $\mathfrak{D}(K) \in \mathcal{D}(X)$ be the Verdier dual of $X$. Let $\mathcal{M}(X)$ be the subcategory of $\mathcal{D}(X)$ whose objects are perverse sheaves. If $\mathcal{E}$ is a local system on $X$ we denote by $\tilde{\mathcal{E}}$ the dual local system. For $K \in \mathcal{D}(X)$ we write ${ }^{p} H^{\cdot}(K)$ instead of $\oplus_{j}{ }^{p} H^{j}(K)$. If $f: X \rightarrow Y$ is a smooth morphism between algebraic varieties with connected fibres of dimension $\delta$, we set $f^{\star} A=f^{*} A[\delta]$ for any $A \in \mathcal{D}(Y)$.

Let $m: H \times Y \rightarrow Y$ be an action of an algebraic group $H$ on an algebraic variety $Y$. For any connected component $C$ of $H$ define $m_{C}: C \times Y \rightarrow Y$ by $(g, y) \mapsto g y$ and $\pi_{C}: C \times Y \rightarrow Y$ by $(g, y) \mapsto y$. Let $K \in \mathcal{M}(Y)$. An $H$-equivariant structure on $K$ is a collection of isomorphisms $\phi_{C}: \pi_{C}^{\star} K \stackrel{\sim}{\rightarrow} m_{C}^{\star} K$ (one for each $C$ ) such that for any two connected components $C, C^{\prime}$ of $H$,

$$
(\tilde{m} \otimes 1)^{\star}\left(\phi_{C C^{\prime}}\right):(\tilde{m} \otimes 1)^{\star}\left(\pi_{C C^{\prime}}^{\star} K\right) \rightarrow(\tilde{m} \otimes 1)^{\star}\left(m_{C C^{\prime}}^{\star} K\right)
$$

is equal to the composition

$$
\begin{aligned}
& (\tilde{m} \otimes 1)^{\star}\left(\pi_{C C^{\prime}}^{\star} K\right) \stackrel{(1)}{=} \tilde{\pi}^{\star}\left(\pi_{C^{\prime}}^{\star} K\right) \stackrel{\beta}{\rightarrow} \tilde{\pi}^{\star}\left(m_{C^{\prime}}^{\star} K\right) \\
& \stackrel{(3)}{\longrightarrow}\left(1 \times m_{C^{\prime}}\right)^{\star}\left(\pi_{C}^{\star} K\right) \stackrel{\gamma}{\rightarrow}\left(1 \times m_{C^{\prime}}\right)^{\star}\left(m_{C}^{\star} K\right) \stackrel{(2)}{\longrightarrow}(\tilde{m} \otimes 1)^{\star}\left(m_{C C^{\prime}}^{\star} K\right)
\end{aligned}
$$


where

$\tilde{m}: C \times C^{\prime} \rightarrow C C^{\prime}$ is $\left(g, g^{\prime}\right) \mapsto g g^{\prime}, \tilde{\pi}: C \times C^{\prime} \times Y \rightarrow C^{\prime} \times Y$ is $\left(g, g^{\prime}, y\right) \mapsto\left(g^{\prime}, y\right)$; the equality (1) comes from $\pi_{C^{\prime}} \tilde{\pi}=\pi_{C C^{\prime}}(\tilde{m} \times 1): C \times C^{\prime} \times Y \rightarrow Y$;

the equality (2) comes from $m_{C C^{\prime}}\left(1 \times m_{C^{\prime}}\right)=m_{C C^{\prime}}(\tilde{m} \times 1): C \times C^{\prime} \times Y \rightarrow Y$; the equality (3) comes from $m_{C^{\prime}} \tilde{\pi}=\pi_{C}\left(1 \times m_{C^{\prime}}\right): C \times C^{\prime} \times Y \rightarrow Y$;

$\beta=\tilde{\pi}^{\star} \phi_{C^{\prime}}, \gamma=\left(1 \times m_{C^{\prime}}\right)^{\star} \phi_{C}$

The notion of $H$-equivariant structure on a local system on $Y$ is defined in the same way (but replace ()$^{\star}$ by ()$\left.^{*}\right)$.

We denote by $\mathcal{M}_{H}(Y)$ the (abelian) category of perverse sheaves on $Y$ endowed with an $H$-equivariant structure; the morphisms are morphisms of perverse sheaves which are compatible with the equivariant structures. Note that any object of $\mathcal{M}_{H}(Y)$ has finite length. If $K \in \mathcal{M}_{H}(Y)$ is semisimple as an object of $\mathcal{M}(Y)$ and $H$ is finite, then $K$ is semisimple as an object of $\mathcal{M}_{H}(Y)$.

Let $f: X \rightarrow Y$ be a morphism compatible with $H$-actions on $X$ and $Y$. If $K \in \mathcal{M}_{H}(Y)$, then ${ }^{p} H^{i}\left(f^{*} K\right)$ is naturally an object of $\mathcal{M}_{H}(X)$. If $K^{\prime} \in \mathcal{M}_{H}(X)$, then ${ }^{p} H^{i}\left(f_{!} K^{\prime}\right)$ is naturally an object of $\mathcal{M}_{H}(Y)$. Similarly, if $\mathcal{E}$ is a local system on $Y$ with a given $H$-equivariant structure, then the local system $f^{*} \mathcal{E}$ on $X$ has an induced $H$-equivariant structure.

If $Y_{0}$ is a locally closed smooth $H$-stable subvariety of pure dimension of $Y$ and $\mathcal{F}$ is an $H$-equivariant local system on $Y_{0}$ we denote by $\mathcal{F}^{\sharp}$ the complex $\operatorname{IC}\left(\bar{Y}_{0}, \mathcal{F}\right)$ (where $\bar{Y}_{0}$ is the closure of $Y_{0}$ in $Y$ ) extended by 0 on $Y-\bar{Y}_{0}$. Then $\mathcal{F}^{\sharp}\left[\operatorname{dim} Y_{0}\right]$ is an object of $\mathcal{M}_{H}(Y)$.

If $K$ is a simple object of $\mathcal{M}_{H}(Y)$, then there exists $Y_{0}, \mathcal{F}$ as above so that the connected components of $Y_{0}$ are permuted transitively by the $H$-action and $K=\mathcal{F}^{\sharp}\left[\operatorname{dim} Y_{0}\right]$. Note that $K$ is not necessarily simple as an object of $\mathcal{M}(Y)$. If $K, K^{\prime}$ are objects of $\mathcal{M}_{H}(Y)$ we write $K \dashv_{\Gamma} K^{\prime}$ if $K$ is isomorphic to a subquotient of $K^{\prime}$.

If $K \in \mathcal{M}_{H}(Y)$, then $\mathfrak{D}(K)$ is naturally an object of $\mathcal{M}_{H}(Y)$.

If $H$ is connected, then $K$ has at most one $H$-equivariant structure.

Now assume that $H$ is finite. Let $m_{g}: Y \rightarrow Y, y \mapsto g y$. An $H$-equivariant structure on $K$ is a collection of isomorphisms $\phi_{g}: K \rightarrow m_{g}^{*} K$ (one for each $g \in H$ ) such that for any $g, g^{\prime}$ in $H, K \stackrel{\phi_{g g^{\prime}}}{\longrightarrow} K$ is equal to the composition $K \stackrel{\phi_{g^{\prime}}}{\longrightarrow}$ $m_{g^{\prime}}^{*} K \stackrel{m_{g^{\prime}}^{*} \phi_{g}}{\longrightarrow} m_{g g^{\prime}}^{*} K$. The same definition applies to $H$-equivariant structures on local systems on $Y$.

Assume now that $A, A^{\prime}$ are two simple objects of $\mathcal{M}_{H}(Y)$. Let $S=\operatorname{supp}(A)$, $S^{\prime}=\operatorname{supp}\left(A^{\prime}\right)$. Note that the irreducible components of $S$ (resp. $S^{\prime}$ ) are permuted transitively by $H$ hence $S$ (resp. $S^{\prime}$ ) has pure dimmension $d$ (resp. $d^{\prime}$ ). We show:

(a) $H_{c}^{0}\left(Y, A \otimes A^{\prime}\right)^{H}=\overline{\mathbf{Q}}_{l}(-d)$ if $A^{\prime}=\mathfrak{D}(A)$ in $\mathcal{M}_{H}(Y)$ and $H_{c}^{0}\left(Y, A \otimes A^{\prime}\right)^{H}=0$ if $A^{\prime} \not \mathfrak{D}(A)$ in $\mathcal{M}_{H}(Y)$.

Note that there exists an open dense smooth $H$-stable subset $S_{0}$ (resp. $S_{0}^{\prime}$ ) of $S$ (resp. $S^{\prime}$ ) and an $H$-equivariant local system $\mathcal{E}$ (resp. $\mathcal{E}^{\prime}$ ) on $S_{0}$ (resp. $S_{0}^{\prime}$ ) such that $A=\mathcal{E}^{\sharp}[d], \mathfrak{D}(A)=\breve{\mathcal{E}}^{\sharp}[d], A^{\prime}=\mathcal{E}^{\sharp}\left[d^{\prime}\right]$. By an argument as in [L2, II, 7.4] we see that $H_{c}^{0}\left(Y, A \otimes A^{\prime}\right)=0$ if $S \neq S^{\prime}$. In the rest of the proof we assume that $S=S^{\prime}$. Then we can also assume that $S_{0}=S_{0}^{\prime}$. Again by the argument in [L2, II, 7.4] we see that $H_{c}^{0}\left(Y, A \otimes A^{\prime}\right)=H_{c}^{2 d}\left(S_{0}, \mathcal{E} \otimes \mathcal{E}^{\prime}\right)$. By Poincaré duality this can be identified with $H^{0}\left(S_{0}, \check{\mathcal{E}} \otimes \check{\mathcal{E}}^{\prime}\right)^{*}(-d)$. It remains to describe $H^{0}\left(S_{0}, \check{\mathcal{E}} \otimes \check{\mathcal{E}}^{\prime}\right)^{H}$. This is the vector space of homomorphisms of local systems $\mathcal{E}^{\prime} \rightarrow \check{\mathcal{E}}$ which are compatible 
with the $H$-equivariant structures. This is the same as the space of morphisms from $A^{\prime}$ to $\mathfrak{D}(A)$ in $\mathcal{M}_{H}(Y)$ which is $\overline{\mathbf{Q}}_{l}$ if $A^{\prime}=\mathfrak{D}(A)$ and is 0 if $A^{\prime} \neq \mathfrak{D}(A)$.

Let $E$ be a finite dimensional $\overline{\mathbf{Q}}_{l}$-vector space and let $r: H \rightarrow G L(E)$ be a homomorphism. We regard $E$ as an $H$-equivariant local system over a point in an obvious way. If $X$ is an algebraic variety with $H$-action, we denote by $\epsilon: X \rightarrow$ point the obvious map and we set $E_{X}=\epsilon^{*} E$; this is naturally an $H$-equivariant local system on $X$ (since $\epsilon$ is compatible with the $H$-action on $X$ and the trivial $H$-action on the point).

1.7. For any torus $T^{\prime}$ let $\mathcal{S}\left(T^{\prime}\right)$ be the category whose objects are the local systems of rank 1 on $T^{\prime}$ that are equivariant for the transitive $T^{\prime}$-action $z: t \mapsto z^{n} t$ on $T^{\prime}$ for some $n \in \mathbf{Z}_{>0}$ invertible in $\mathbf{k}$.

1.8. Let $f: T^{\prime} \rightarrow T^{\prime \prime}$ be a morphism of tori and let $\mathcal{L} \in \mathcal{S}\left(T^{\prime \prime}\right)$. We show that the following two conditions are equivalent:

(i) $f^{*} \mathcal{L} \cong \overline{\mathbf{Q}}_{l}$;

(ii) $\mathcal{L}$ is equivariant for the $T^{\prime}$-action $t^{\prime}: t^{\prime \prime} \mapsto f\left(t^{\prime}\right) t^{\prime \prime}$ on $T^{\prime \prime}$.

We can find $\kappa \in \operatorname{Hom}\left(T^{\prime \prime}, \mathbf{k}^{*}\right)$ and $\mathcal{E} \in \mathcal{S}\left(\mathbf{k}^{*}\right)$ such that $\mathcal{L} \cong \kappa^{*}(\mathcal{E})$. If the result holds for $\kappa f, \mathcal{E}$ instead of $f, \mathcal{L}$, then it also holds for $f, \mathcal{L}$. Thus we may assume that $T^{\prime \prime}=\mathbf{k}^{*}$. We can assume that $T^{\prime} \neq\{1\}$. Let $T_{0}$ be a codimension 1 subtorus of $T^{\prime}$ contained in ker $f$. Then $f$ induces a homomorphism $f^{\prime}: T^{\prime} / T_{0} \rightarrow T^{\prime \prime}$. If the result holds for $f^{\prime}, \mathcal{L}$ instead of $f, \mathcal{L}$, then it also holds for $f, \mathcal{L}$. Thus we may assume that $T^{\prime}=T^{\prime \prime}=\mathbf{k}^{*}$. In this case the result is immediate.

1.9. In this subsection we assume that $\mathbf{k}$ is an algebraic closure of a finite field $\mathbf{F}_{q}$. Let $F^{\prime}: T \rightarrow T$ be the Frobenius map for some $\mathbf{F}_{q}$-rational structure on the torus $T$. Let $T^{F^{\prime}}=\left\{t \in T ; F^{\prime}(t)=t\right\}$. The following three sets coincide:

(i) the set of $\mathcal{L} \in \mathcal{S}(T)$ (up to isomorphism) such that $\mathcal{L}$ is $T$-equivariant for the $T$-action $t_{0}: t \mapsto t_{0} t F^{\prime}\left(t_{0}\right)^{-1}$ on $T$;

(ii) the set of $\mathcal{L} \in \mathcal{S}(T)$ (up to isomorphism) such that $F^{*} \mathcal{L} \cong \mathcal{L}$;

(iii) the set of $\mathcal{L} \in \mathcal{S}(T)$ (up to isomorphism) such that $\mathcal{L}$ is a direct summand of $L_{!} \overline{\mathbf{Q}}_{l}$ where $L: T \rightarrow T$ is $t \mapsto t F^{\prime}\left(t^{-1}\right)$;

moreover, they are in a natural bijection with

(iv) the set of characters $\operatorname{Hom}\left(T^{F^{\prime}}, \overline{\mathbf{Q}}_{l}^{*}\right)$.

For $\mathcal{L} \in \mathcal{S}(T)$ we have $L^{*} \mathcal{L} \cong \mathcal{L} \otimes F^{*}(\check{\mathcal{L}})$. Hence $\mathcal{L}$ satisfies (ii) if and only if it satisfies $L^{*} \mathcal{L} \cong \overline{\mathbf{Q}}_{l}$. The last condition is clearly equivalent to the condition in (iii) and by 1.8 it is also equivalent to the condition in (i).

If $L$ is as in (iii), $T^{F^{\prime}}$ acts in an obvious way on $L_{!} \overline{\mathbf{Q}}_{l}$ and we have $L_{!} \overline{\mathbf{Q}}_{l}=$ $\oplus_{\chi \in \operatorname{Hom}\left(T^{F^{\prime}}, \overline{\mathbf{Q}}_{l}^{*}\right)} L_{!}^{\chi} \overline{\mathbf{Q}}_{l}$ where $L_{!}^{\chi} \overline{\mathbf{Q}}_{l}$ is the subsheaf on which $T^{F^{\prime}}$ according to $\chi$. It is clear that $\chi \mapsto L_{!}^{\chi} \overline{\mathbf{Q}}_{l}$ defines a bijection between the sets (iv) and (iii). The inverse of this bijection can be described as follows. Let $\mathcal{L}$ be as in (i). By restriction of the equivariant $T$-structure we obtain an equivariant $T^{F^{\prime}}$-structure on $\mathcal{L}$. Since $T^{F^{\prime}}$ acts trivially on $T$, it acts naturally on the stalk of $\mathcal{L}$ at 1 ; this action is via a character $\chi_{\mathcal{L}}: T^{\prime F} \rightarrow \overline{\mathbf{Q}}_{l}^{*}$. Now $\mathcal{L} \mapsto \chi_{\mathcal{L}}$ is the inverse of the bijection above.

1.10. Let $\mathcal{L} \in \mathcal{S}(T)$. Let $R_{\mathcal{L}}=\left\{\alpha \in R ; \check{\alpha}^{*} \mathcal{L} \cong \overline{\mathbf{Q}}_{l}\right\}$. Then $R_{\mathcal{L}}$ is a root system and $R_{\mathcal{L}}^{+}=R_{\mathcal{L}} \cap R^{+}$is a set of positive roots for $R_{\mathcal{L}}$. Let $\Pi_{\mathcal{L}}$ be the unique set of simple roots of $R_{\mathcal{L}}$ such that $\Pi_{\mathcal{L}} \subset R_{\mathcal{L}}^{+}$. Let $W_{\mathcal{L}}$ be the subgroup of $W$ generated by the reflections with respect to the roots in $R_{\mathcal{L}}$. Let $\mathbf{I}_{\mathcal{L}}$ be the set of 
reflections with respect to the roots in $\Pi_{\mathcal{L}}$. Then $\left(W_{\mathcal{L}}, \mathbf{I}_{\mathcal{L}}\right)$ is a Coxeter group. Let $\check{R}_{\mathcal{L}}=\left\{\check{\alpha} ; \alpha \in R_{\mathcal{L}}\right\}$.

\section{The VARIETY $\mathcal{Z}^{\mathbf{s}}$ AND THE LOCAL SYSTEM $\overline{\mathcal{L}}$}

2.1. In this and the next subsection we assume that $\mathbf{I}$ consists of a single element s. Let $a=\check{\alpha}_{s}: \mathbf{k}^{*} \rightarrow T$. To any $\mathcal{L} \in \mathcal{S}(T)$ such that

(a) $a^{*} \mathcal{L} \cong \overline{\mathbf{Q}}_{l}$

we will associate a local system $\underline{\mathcal{L}}$ of rank 1 on $G$.

Case 1. $a$ is an imbedding. We have a diagram $T \stackrel{c}{\rightarrow} T / a\left(\mathbf{k}^{*}\right) \stackrel{d}{\rightarrow} G / G_{d e r} \stackrel{e}{\leftarrow} G$ where $c, e$ are the obvious maps and $d$ is induced by the inclusion $T \subset G$; note that $d$ is an isomorphism. Now $\mathbf{k}^{*}$ acts on $T$ by $x: t \mapsto a(x) t$ and on $T / a\left(\mathbf{k}^{*}\right)$ trivially; $c$ is compatible with the $\mathbf{k}^{*}$-actions. From (a) we see that $\mathcal{L}$ is $\mathbf{k}^{*}$-equivariant. Since $\mathbf{k}^{*}$ acts freely on $T$ there is a well defined local system $\mathcal{L}_{1}$ of rank 1 on $T / a\left(\mathbf{k}^{*}\right)$ such that $\mathcal{L}=c^{*} \mathcal{L}_{1}$. We set $\underline{\mathcal{L}}=e^{*}\left(d^{-1}\right)^{*} \mathcal{L}_{1}$.

Case 2. a is not an imbedding. Then the centre $\mathcal{Z}$ of $G$ is connected, the obvious homomorphism $G_{d e r} \times \mathcal{Z} \rightarrow G$ is an isomorphism and we can identify $G_{\text {der }}=P G L_{2}(\mathbf{k})$ compatibly with the standard épinglages. Thus we can identify $G=P G L_{2}(\mathbf{k}) \times \mathcal{Z}$. Let $G^{\prime}=G L_{2}(\mathbf{k}) \times \mathcal{Z}$ and let $\pi: G^{\prime} \rightarrow G$ be the obvious homomorphism. Let $K=\operatorname{ker} \pi$, a one-dimensional torus. Let $T^{\prime}=\pi^{-1}(T)$. Let $\pi_{0}: T^{\prime} \rightarrow T$ be the restriction of $\pi$. Let $a^{\prime}: \mathbf{k}^{*} \rightarrow T^{\prime}$ be the coroot of $G^{\prime}$ such that $\pi_{0} a^{\prime}=a$. Let $\mathcal{L}^{\prime}=\pi_{0}^{*} \mathcal{L}$. Note that $a^{\prime *} \mathcal{L}^{\prime} \cong \overline{\mathbf{Q}}_{l}$. Applying the construction in Case 1 to $G^{\prime}, T^{\prime}, \mathcal{L}^{\prime}$ instead of $G, T, \mathcal{L}$ we obtain a local system $\underline{\mathcal{L}}^{\prime}$ on $G^{\prime}$. Now $K$ acts on $T^{\prime}, G^{\prime}, G^{\prime} / G_{d e r}^{\prime}, T^{\prime} / a^{\prime}\left(\mathbf{k}^{*}\right)$ by translation, the analogues of $c, d, e$ for $G^{\prime}$ are compatible with the $K$-action and $\mathcal{L}^{\prime}$ is $K$-equivariant. Hence $\underline{\mathcal{L}}^{\prime}$ is $K$-equivariant. Since $K$ acts freely on $G^{\prime}$ there is a well defined local system $\underline{\mathcal{L}}$ of rank 1 on $G$ such that $\pi^{*} \underline{\mathcal{L}}=\underline{\mathcal{L}}^{\prime}$.

2.2. Define $f: G-B \rightarrow T$ by $f(y)=k(y) \dot{s}^{-1}$ and $f^{1}: B \rightarrow T$ by $f^{1}(y)=k(y)$. We show:

(a) we have canonically $\left.\underline{\mathcal{L}}\right|_{G-B}=f^{*} \mathcal{L}$ and $\left.\underline{\mathcal{L}}\right|_{B}=f^{1 *} \mathcal{L}$.

In the setup of 2.1, assume first that we are in Case 1. Let $j: G-B \rightarrow G$, $h: B \rightarrow G$ be the inclusions. We must show that

$$
j^{*} e^{*}\left(d^{-1}\right)^{*} \mathcal{L}_{1}=f^{*} c^{*} \mathcal{L}_{1}, h^{*} e^{*}\left(d^{-1}\right)^{*} \mathcal{L}_{1}=f^{1 *} c^{*} \mathcal{L}_{1} .
$$

It is enough to show that $d^{-1} e j=c f, d^{-1} e h=c f^{1}$ or that $e j=d c f$ (resp. $e h=d c f^{1}$ ). Both maps take $u t \dot{s} u^{\prime}$ (resp. $u t$ ), where $u, u^{\prime} \in U, t \in T$, to the image of $t$ in $G / G_{d e r}$. (We use that $u, u^{\prime}, \dot{s} \in G_{d e r}$.)

Next we assume that we are in Case 2. Define $\dot{s}^{\prime} \in G^{\prime}$ in terms of the unique épinglage of $G^{\prime}$ compatible under $\pi$ with that of $G$ in the same way that $\dot{s} \in G$ is defined in terms of the épinglage of $G$. Let $B^{\prime}=\pi^{-1}(B)$. Define $f^{\prime}: G^{\prime}-B^{\prime} \rightarrow T^{\prime}$, $f^{\prime 1}: B^{\prime} \rightarrow T^{\prime}$ in terms of $G^{\prime}, B^{\prime}, T^{\prime}, \dot{s}^{\prime}$ in the same way that $f, f^{1}$ are defined in terms of $G, B, T, \dot{s}$. Let $\pi_{s}: G^{\prime}-B^{\prime} \rightarrow G-B, \pi_{1}: B^{\prime} \rightarrow B$ be the restrictions of $\pi$. It is enough to show that

$$
\pi_{s}^{*}\left(\left.\underline{\mathcal{L}}\right|_{G-B}\right)=\pi_{s}^{*} f^{*} \mathcal{L}, \quad \pi_{1}^{*}\left(\left.\underline{\mathcal{L}}\right|_{B}\right)=\pi_{1}^{*} f^{1 *} \mathcal{L} .
$$

We have

$$
\pi_{s}^{*}\left(\left.\underline{\mathcal{L}}\right|_{G-B}\right)=\left.\underline{\mathcal{L}}^{\prime}\right|_{G^{\prime}-B^{\prime}}, \pi_{s}^{*} f^{*} \mathcal{L}=f^{\prime *} \mathcal{L}^{\prime}, \pi_{1}^{*}\left(\left.\underline{\mathcal{L}}\right|_{B}\right)=\left.\underline{\mathcal{L}}^{\prime}\right|_{B^{\prime}}, \pi_{1}^{*} f^{1 *} \mathcal{L}=f^{\prime 1 *} \mathcal{L}^{\prime}
$$


Hence it is enough to show that $\left.\underline{\mathcal{L}}^{\prime}\right|_{G^{\prime}-B^{\prime}}=f^{\prime *} \mathcal{L}^{\prime},\left.\underline{\mathcal{L}}^{\prime}\right|_{B^{\prime}}=f^{\prime 1 *} \mathcal{L}^{\prime}$. But these are known from Case 1 applied to $G^{\prime}, \mathcal{L}^{\prime}$ instead of $G, \mathcal{L}$. This proves (a).

2.3. We return to the general case. Let $s \in \mathbf{I}$. Let $P=P_{s}$. Let $\pi_{P}: P \rightarrow P / U_{P}$ be the obvious map. Note that $P / U_{P}$ inherits an épinglage from $G$ and that $T$, identified with its image under $\pi_{P}$ is a maximal torus of $P / U_{P}$. To any $\mathcal{L} \in \mathcal{S}(T)$ such that $\check{\alpha}_{s}^{*} \mathcal{L} \cong \overline{\mathbf{Q}}_{l}$ we associate a local system of rank 1 on $P$, namely the inverse image of the local system $\underline{\mathcal{L}}$ on $P / U_{P}$ (see 2.1 ) under $\pi_{P}$; this local system on $P$ is denoted again by $\underline{\mathcal{L}}$.

Define $f_{s}: P-B \rightarrow T$ by $f_{s}(y)=k(y) \dot{s}^{-1}$ and $f_{s}^{1}: B \rightarrow T$ by $f_{s}^{1}(y)=k(y)$. From 2.2(a) we deduce by taking inverse image under $\pi_{P}$ :

(a) we have canonically $\left.\underline{\mathcal{L}}\right|_{P-B}=f_{s}^{*} \mathcal{L}$ and $\left.\underline{\mathcal{L}}\right|_{B}=f_{s}^{1 *} \mathcal{L}$, (as local systems over subsets of $P$ ).

2.4. Let $\mathbf{s}=\left(s_{1}, s_{2}, \ldots, s_{r}\right)$ be a sequence in $\mathbf{I}$. Let $\mathcal{L} \in \mathcal{S}(T)$. Let

$$
\mathcal{I}_{\mathbf{s}}=\left\{i \in[1, r] ; s_{1} s_{2} \ldots s_{i} \ldots s_{2} s_{1} \in W_{\mathcal{L}}\right\} .
$$

Let

$$
\mathcal{Y}=\left\{\left(y_{i}\right) \in G^{[1, r]} ; y_{i} \in P_{s_{i}}\left(i \in \mathcal{I}_{\mathbf{s}}\right), y_{i} \in P_{s_{i}}-B\left(i \in[1, r]-\mathcal{I}_{\mathbf{s}}\right)\right\} .
$$

For $i \in[1, r]$ we define $f_{s_{i}}: P_{s_{i}}-B \rightarrow T$ by $f_{s_{i}}(y)=k(y) \dot{s}_{i}^{-1}$ and $f_{s_{i}}^{1}: B \rightarrow T$ by $f_{s_{i}}^{1}(y)=k(y)$. We have obvious projections $p_{i}: \mathcal{Y} \rightarrow P_{s_{i}}\left(i \in \mathcal{I}_{\mathbf{s}}\right), p_{i}: \mathcal{Y} \rightarrow$ $P_{s_{i}}-B\left(i \in[1, r]-\mathcal{I}_{\mathbf{s}}\right)$. Let

with

$$
\underline{\underline{\mathcal{L}}}=\otimes_{i \in[1, r]} \mathcal{F}_{i}
$$

$$
\mathcal{F}_{i}=p_{i}^{*} \underline{s_{i-1}^{*} \ldots s_{2}^{*} s_{1}^{*} \mathcal{L}} \text { for } i \in \mathcal{I}_{\mathbf{s}}, \mathcal{F}_{i}=p_{i}^{*} f_{s_{i}}^{*} s_{i-1}^{*} \ldots s_{2}^{*} s_{1}^{*} \mathcal{L} \text { for } i \in[1, r]-\mathcal{I}_{\mathbf{s}} .
$$

Here $\mathcal{F}_{i}, \underline{\underline{L}}$ are local systems on $\mathcal{Y}$. Note that if $i \in \mathcal{I}_{\mathbf{s}}$, then the local system $s_{i-1}^{*} \ldots s_{2}^{*} s_{1}^{*} \mathcal{L}$ on $P_{s_{i}}$ is well defined (see 2.1) since $\check{\alpha}_{s_{i}}^{*}\left(s_{i-1}^{*} \ldots s_{2}^{*} s_{1}^{*} \mathcal{L}\right) \cong \overline{\mathbf{Q}}_{l}$.

For any $\mathcal{J} \subset \mathcal{I}_{\mathbf{s}}$, let

$$
\mathcal{Y}^{\mathcal{J}}=\left\{\left(y_{i}\right) \in G^{[1, r]} ; y_{i} \in P_{s_{i}}-B(i \in[1, r]-\mathcal{J}), y_{i} \in B(i \in \mathcal{J})\right\}
$$

and let $\mathbf{s}_{\mathcal{J}}=\left(s_{1}^{\prime}, s_{2}^{\prime}, \ldots, s_{r}^{\prime}\right)$ where $s_{i}^{\prime}=s_{i}$ if $i \in[1, r]-\mathcal{J}, s_{i}^{\prime}=1$ if $i \in \mathcal{J}$. Define $f^{\mathcal{J}}: \mathcal{Y}^{\mathcal{J}} \rightarrow T$ by $\left(y_{i}\right) \mapsto k\left(y_{1}\right) k\left(y_{2}\right) \ldots k\left(y_{r}\right)\left[\mathbf{s}_{\mathcal{J}}\right]^{\bullet-1}$. We show:

(a) We have canonically $\left.\underline{\underline{\mathcal{L}}}\right|_{\mathcal{Y}^{\mathcal{J}}}=\left(f^{\mathcal{J}}\right)^{*} \mathcal{L}$.

We have obvious projections $p_{i}^{\prime}: \mathcal{Y}^{\mathcal{J}} \rightarrow P_{s_{i}}-B(i \in[1, r]-\mathcal{J}), p_{i}^{\prime}: \mathcal{Y}^{\mathcal{J}} \rightarrow$ $B(i \in \mathcal{J})$. Using 2.3(a) we have canonically $\left.\underline{\underline{\mathcal{L}}}\right|_{\mathcal{Y} \mathcal{J}}=\otimes_{i \in[1, r]} \mathcal{F}_{i}^{\prime}$ where

$$
\mathcal{F}_{i}^{\prime}=p_{i}^{\prime *} f_{s_{i}}^{*} s_{i-1}^{*} \ldots s_{2}^{*} s_{1}^{*} \mathcal{L} \text { for } i \in[1, r]-\mathcal{J}, \mathcal{F}_{i}^{\prime}=p_{i}^{\prime *} f_{s_{i}}^{1 *} s_{i-1}^{*} \ldots s_{2}^{*} s_{1}^{*} \mathcal{L} \text { for } i \in \mathcal{J} .
$$

We define $\tilde{f}: \mathcal{Y}^{\mathcal{J}} \rightarrow T^{[1, r]}$ by $\tilde{f}=\left(\tilde{f}_{i}\right)$ where for $i \in[1, r], \tilde{f}_{i}: \mathcal{Y}^{\mathcal{J}} \rightarrow T$ is given by

$$
\tilde{f}_{i}=s_{1} s_{2} \ldots s_{i-1} f_{s_{i}} p_{i}^{\prime} \text { for } i \in[1, r]-\mathcal{J}, \tilde{f}_{i}=s_{1} s_{2} \ldots s_{i-1} f_{s_{i}}^{1} p_{i}^{\prime} \text { for } i \in \mathcal{J} .
$$

Then $\mathcal{F}_{i}^{\prime}=\tilde{f}_{i}^{*} \mathcal{L}$ for $i \in[1, r]$ and

$$
\otimes_{i \in[1, r]} \mathcal{F}_{i}^{\prime}=\tilde{f}^{*}(\mathcal{L} \otimes \mathcal{L} \otimes \ldots \otimes \mathcal{L}) .
$$

For $y \in \mathcal{Y}^{\mathcal{J}}$ we have $f^{\mathcal{J}}(y)=\tilde{f}_{1}(y) \tilde{f}_{2}(y) \ldots \tilde{f}_{r}(y)=m \tilde{f}(y)$ where $m: T^{[1, r]} \rightarrow T$ is multiplication. Hence $\left(f^{\mathcal{J}}\right)^{*} \mathcal{L}=\tilde{f}^{*} m^{*} \mathcal{L}$. It is then enough to show that $m^{*} \mathcal{L}=$ $\mathcal{L} \otimes \mathcal{L} \otimes \ldots \otimes \mathcal{L}$; this is a known property of any local system in $\mathcal{S}(T)$. This proves (a). 
Let

$$
Y=\left\{\left(y_{i}\right) \in G^{[1, r]} ; y_{i} \in P_{s_{i}}(i \in[1, r])\right\} .
$$

Note that $\mathcal{Y}$ is an open dense subset of $Y$. Hence $\operatorname{IC}(Y, \underline{\underline{\mathcal{L}}})$ is well defined. We show:

$$
\left.I C(Y, \underline{\underline{\mathcal{L}}})\right|_{Y-\mathcal{Y}}=0
$$

For any $j \in[1, r]-\mathcal{I}_{\mathbf{s}}$ let

$$
\Delta_{j}=\left\{\left\{\left(y_{i}\right) \in G^{[1, r]} ; y_{i} \in P_{s_{i}}(i \in[1, r]-\{j\}), y_{j} \in B\right\} .\right.
$$

Clearly, $\left\{\Delta_{j}, j \in[1, r]-\mathcal{I}_{\mathbf{s}}\right\}$ are smooth divisors with normal crossings in the smooth variety $Y$. Using [L2, I, 1.6] we see that it suffices to prove the following statement.

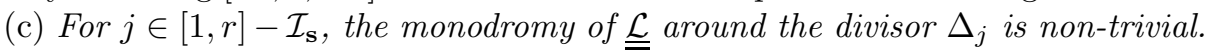

We define a cross-section $\xi: \mathbf{k} \rightarrow Y$ to $\Delta_{j}$ in $Y$ by

$$
\xi(a)=\left(\dot{s}_{1}, \ldots, \dot{s}_{j-1}, y_{s_{j}}(-a), \dot{s}_{j+1}, \ldots, \dot{s}_{r}\right) .
$$

We have $\xi(0) \in \Delta_{j}, \xi(a) \in \mathcal{Y}$ for $a \in \mathbf{k}^{*}$. Let $\xi^{\prime}: \mathbf{k}^{*} \rightarrow \mathcal{Y}$ be the restriction of $\xi$. It is enough to show that $\xi^{\prime *} \underline{\underline{\mathcal{L}}} \neq \overline{\mathbf{Q}}_{l}$ or, with notation in 2.4 , that

$$
\xi^{*} p_{j}^{*} f_{s_{j}}^{*} s_{j-1}^{*} \ldots s_{2}^{*} s_{1}^{*} \mathcal{L} \neq \overline{\mathbf{Q}}_{l}
$$

or that

$$
\left(s_{1} s_{2} \ldots s_{j-1} f_{s_{j}} p_{j} \xi^{\prime}\right)^{*} \mathcal{L} \neq \overline{\mathbf{Q}}_{l},\left(s_{1} s_{2} \ldots s_{j-1} \check{\alpha}_{s_{j}}\right)^{*} \mathcal{L} \neq \overline{\mathbf{Q}}_{l} .
$$

(We have $f_{s_{j}} p_{j} \xi^{\prime}(a)=k\left(y_{s_{j}}(-a)\right) \dot{s}_{j}^{-1}=\check{\alpha}_{s_{j}}(a)$.) This follows from the fact that $j \notin \mathcal{I}_{\text {s. }}$. This proves (c) and hence (b).

(A similar result with a similar proof appears in [L6, VI, 28.10(b)].)

In the remainder of this paper we assume that $\mathbf{k}$ is an algebraic closure of $\mathbf{F}_{q}$, a finite field with $q$ elements and that we are given a fixed $\mathbf{F}_{q}$-rational structure on $G$ such that $B$ and $T$ are defined over $\mathbf{F}_{q}$. Let $F: G \rightarrow G$ be the corresponding Frobenius map. We set

$$
\Gamma=\{g \in G ; F(g)=g\} .
$$

Now $F: G \rightarrow G$ induces an isomorphism $F: T \rightarrow T$. For $w \in W$ we write $\mathcal{L} \in \mathcal{S}(T)^{w F}$ instead of " $\mathcal{L} \in \mathcal{S}(T)$ and $(w F)^{*} \mathcal{L} \cong \mathcal{L}$ ".

Define $F_{0}: T \rightarrow T$ by $t \mapsto t^{q}$. For any $t \in T$ we have $F(t)=F_{0}(\mathbf{c}(t))=$ $\mathbf{c}\left(F_{0}(t)\right)$ where $\mathbf{c}: T \rightarrow T$ is a well defined automorphism. Then $w \mapsto \mathbf{c} w \mathbf{c}^{-1}$ is an automorphism of $W$ denoted also by $w \mapsto \mathbf{c}(w)$. This restricts to a bijection $\mathbf{I} \stackrel{\sim}{\rightarrow} \mathbf{I}$. Let

We show:

$$
\underline{B}:=\left\{\left(b_{i}\right) \in B^{[0, r]} ; k\left(b_{r}^{-1} F\left(b_{0}\right)\right)=1\right\} .
$$

(d) If $\mathcal{L} \in \mathcal{S}(T)^{[\mathbf{s}] F}$, then the local system $\underline{\underline{\mathcal{L}}}$ is equivariant for the $\underline{B}$-action

$$
\left(b_{0}, b_{1}, \ldots, b_{r}\right):\left(y_{1}, y_{2}, \ldots y_{r}\right) \mapsto\left(b_{0} y_{1} b_{1}^{-1}, b_{1} y_{2} b_{2}^{-1}, \ldots, b_{r-1} y_{r} b_{r}^{-1}\right)
$$

on $\mathcal{Y}$.

By (a), the restriction of $\underline{\underline{\mathcal{L}}}$ to the $\underline{B}$-stable open dense subset $\mathcal{Y}^{\emptyset}$ of $\mathcal{Y}$ is $\left(f^{\emptyset}\right)^{*} \mathcal{L}$. Since $\mathcal{Y}$ is smooth, it is enough to show that the local system $\left(f^{\emptyset}\right)^{*} \mathcal{L}$ on $\mathcal{Y}^{\emptyset}$ is $\underline{B}$-equivariant. Now $\underline{B}$ acts on $T$ by $\left(b_{0}, b_{1}, \ldots, b_{r}\right): t \mapsto k\left(b_{0}\right)^{-1} t\left([\mathbf{s}] F\left(b_{0}\right)\right)$ and $f^{\emptyset}: \mathcal{Y}^{\emptyset} \rightarrow T$ is compatible with the $\underline{B}$-actions. Hence it is enough to show that $\mathcal{L}$ is $\underline{B}$-equivariant. An equivalent statement is that $\mathcal{L}$ is $T$-equivariant for the $T$-action $\bar{t}_{0}: t \mapsto t_{0}^{-1} t\left([\mathbf{s}] F\left(t_{0}\right)\right)$. This follows from our assumption on $\mathcal{L} ;(\mathrm{d})$ is proved. 
2.5. Let $\mathbf{w}=\left(w_{1}, w_{2}, \ldots, w_{r}\right)$ be a sequence in $W$. Let

$$
\begin{aligned}
& Z^{\mathbf{w}}=\left\{\left(B_{i}\right) \in \mathcal{B}^{[0, r]} ; \operatorname{pos}\left(B_{i-1}, B_{i}\right)=w_{i}(i \in[1, r]), B_{r}=F\left(B_{0}\right)\right\}, \\
& \dot{Z}=\left\{\left(g_{i} U\right) \in(G / U)^{[0, r]} ; k\left(g_{i-1}^{-1} g_{i}\right)=\dot{w}_{i}(i \in[1, r]), g_{r}^{-1} F\left(g_{0}\right) \in U\right\} .
\end{aligned}
$$

Let

$$
\mathfrak{T}=\left\{\left(t_{i}\right) \in T^{[0, r]} ; t_{i}=w_{i}^{-1}\left(t_{i-1}\right)(i \in[1, r]), t_{r}=F\left(t_{0}\right)\right\},
$$

a finite subgroup of $T^{[0, r]}$ which may be identified via $\left(t_{i}\right) \mapsto t_{0}$ with $T^{F^{\prime}}$ where $F^{\prime}: T \rightarrow T$ is $t \mapsto[\mathbf{w}] F(t)$. The free $\mathfrak{T}$-action $\left(t_{i}\right):\left(g_{i} U\right) \mapsto\left(g_{i} t_{i}^{-1} U\right)$ on $\dot{Z}$ makes $\dot{Z}$ into a principal $\mathfrak{T}$-bundle over $Z^{\mathbf{w}}$ via the map $f: \dot{Z} \rightarrow Z^{\mathbf{w}},\left(g_{i} U\right) \mapsto\left(g_{i} B g_{i}^{-1}\right)$. Now $f_{!} \overline{\mathbf{Q}}_{l}$ is a local system on $Z^{\mathbf{w}}$ with a free action of $\mathfrak{T}=T^{F^{\prime}}$ on each stalk. We have $f_{!} \overline{\mathbf{Q}}_{l}=\oplus_{\chi \in \operatorname{Hom}\left(T^{F^{\prime}}, \overline{\mathbf{Q}}_{l}^{*}\right)} f_{!}^{\chi} \overline{\mathbf{Q}}_{l}$ where $f_{!}^{\chi} \overline{\mathbf{Q}}_{l}$ is the subsheaf of $f_{!} \overline{\mathbf{Q}}_{l}$ on which $T^{F^{\prime}}$ acts according to $\chi$.

Now $\Gamma$ acts on $Z^{\mathbf{w}}$ by $g:\left(B_{i}\right) \mapsto\left(g B_{i} g^{-1}\right)$, and on $\dot{Z}$ by $g:\left(g_{i} U\right) \mapsto\left(g g_{i} U\right)$. This last action commutes with the $\mathfrak{T}$-action. Hence $f_{!} \overline{\mathbf{Q}}_{l}$ has a natural $\Gamma$-equivariant structure and each $f_{!}^{\chi} \overline{\mathbf{Q}}_{l}$ inherits a $\Gamma$-equivariant structure from $f_{!} \overline{\mathbf{Q}}_{l}$.

We now give an alternative construction of the local systems $f_{!}^{\chi} \overline{\mathbf{Q}}_{l}$. Let

$$
\underline{Z}=\left\{\left(g_{i} U\right) \in(G / U)^{[0, r]} ; g_{i-1}^{-1} g_{i} \in B \dot{w}_{i} B(i \in[1, r]), g_{r}^{-1} F\left(g_{0}\right) \in U\right\} .
$$

Define $\gamma: \underline{Z} \rightarrow Z^{\mathbf{w}}$ by $\left(g_{i} U\right) \mapsto\left(g_{i} B g_{i}^{-1}\right)$. Define $\pi_{\mathbf{w}}: \underline{Z} \rightarrow T$ by

$$
\left(g_{i} U\right) \mapsto k\left(g_{0}^{-1} g_{1}\right) k\left(g_{1}^{-1} g_{2}\right) \ldots k\left(g_{r-1}^{-1} g_{r}\right)[\mathbf{w}]^{\bullet-1} .
$$

The torus $\underline{T}:=\left\{\left(t_{i}\right) \in T^{[0, r]} ; t_{r}=F\left(t_{0}\right)\right\}$ acts on $\underline{Z}$ by $\left(t_{i}\right):\left(g_{i} U\right) \mapsto\left(g_{i} t_{i}^{-1} U\right)$ and on $T$ by $\left(t_{i}\right): t \mapsto t_{0} t\left([\mathbf{w}] F\left(t_{0}^{-1}\right)\right)$. These actions are compatible with $\pi_{\mathbf{w}}$. Let $\mathcal{L} \in \mathcal{S}(T)^{[\mathbf{w}] F}$. By 1.8, $\mathcal{L}$ is equivariant for the $\underline{T}$-action on $T$. Hence $\pi_{\mathrm{w}}^{*} \mathcal{L}$ is equivariant for the (free) $\underline{T}$-action on $\underline{Z}$. Hence $\pi_{\mathrm{w}}^{*} \mathcal{L}=\gamma^{*} \mathcal{L}_{\mathrm{w}}$ for a well defined local system $\mathcal{L}_{\mathrm{w}}$ on $Z^{\mathbf{w}}$.

Now $\Gamma$ acts on $\underline{Z}$ by $g:\left(g_{i} U\right) \mapsto\left(g g_{i} U\right)$ and on $T$ trivially. Also, $\mathcal{L}$ has a natural $\Gamma$-equivariant structure in which $\Gamma$ acts trivially on each stalk of $\mathcal{L}$. Since $\pi_{\mathrm{w}}$ is compatible with the $\Gamma$-actions it follows that $\pi_{\mathrm{w}}^{*} \mathcal{L}$ has a natural $\Gamma$-equivariant structure. Since $\gamma$ is compatible with the $\Gamma$-actions it follows that $\mathcal{L}_{\mathrm{w}}$ has a natural $\Gamma$-equivariant structure.

Now assume that $\mathcal{L}$ and $\chi \in \operatorname{Hom}\left(T^{F^{\prime}}, \overline{\mathbf{Q}}_{l}^{*}\right)$ correspond to each other as in 1.9. Thus we assume that $\mathcal{L}=L_{!}^{\chi} \overline{\mathbf{Q}}_{l}$ where $L: T \rightarrow T$ is as in 1.9. We show that

$$
\mathcal{L}_{\mathbf{w}}=f_{!}^{\chi} \overline{\mathbf{Q}}_{l} \text {. }
$$

Since $\gamma$ is smooth with connected fibres it is enough to show that $\pi_{\mathbf{w}}^{*} \mathcal{L}=\gamma^{*} f_{!}^{\chi} \overline{\mathbf{Q}}_{l}$. Let

$$
\begin{aligned}
\mathfrak{P}= & \left\{\left(g_{i} U, \tau_{i}\right) \in(G / U \times T)^{[0, r]} ; k\left(\tau_{i-1} g_{i-1}^{-1} g_{i} \tau_{i}^{-1}\right)=\dot{w}_{i}(i \in[1, r]),\right. \\
& \left.\tau_{r}=F\left(\tau_{0}\right), g_{r}^{-1} F\left(g_{0}\right) \in U\right\} .
\end{aligned}
$$

Define $f^{\prime}: \mathfrak{P} \rightarrow \underline{Z}$ by $\left(g_{i} U, \tau_{i}\right) \mapsto\left(g_{i} U\right)$ and $\gamma^{\prime}: \mathfrak{P} \rightarrow \dot{Z}$ by $\left(g_{i} U, \tau_{i}\right) \mapsto\left(g_{i} \tau_{i}^{-1} U\right)$. Define $\pi^{\prime}: \mathfrak{P} \rightarrow T$ by $\left(g_{i} U, \tau_{i}\right) \mapsto \tau_{0}$. Now $\mathfrak{T}$ acts on $\mathfrak{P}$ by $\left(t_{i}\right):\left(g_{i} U, \tau_{i}\right) \mapsto$ $\left(g_{i} U, t_{i} \tau_{i}\right)$, making $f^{\prime}$ into a principal $\mathfrak{T}$-bundle. We have a cartesian diagram of 
principal $\mathfrak{T}=T^{F^{\prime}}$ bundles:

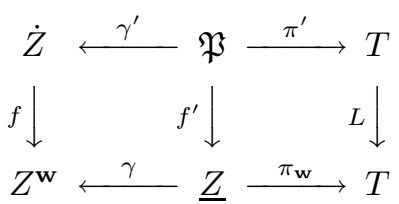

It follows that $\gamma^{*}\left(f_{!} \overline{\mathbf{Q}}_{l}\right)=f_{!}^{\prime} \overline{\mathbf{Q}}_{l}=\pi_{\mathbf{w}}^{*}\left(L_{!} \overline{\mathbf{Q}}_{l}\right)$, and taking $\chi$-eigenspaces: $\gamma^{*}\left(f_{!}^{\chi} \overline{\mathbf{Q}}_{l}\right)=$ $f_{!}^{\prime} \chi \overline{\mathbf{Q}}_{l}=\pi_{\mathbf{w}}^{*}\left(L_{!}^{\chi} \overline{\mathbf{Q}}_{l}\right)$. Thus, $\gamma^{*}\left(f_{!}^{\chi} \overline{\mathbf{Q}}_{l}\right)=\pi_{\mathbf{w}}^{*} \mathcal{L}$, as required.

From the definitions we see that (a) is compatible with the $\Gamma$-equivariant structures.

When $\mathbf{w}=(w)$ is a one term sequence with $w \in W$ we can identify $Z^{\mathbf{w}}$ with

$$
\mathcal{B}_{w}=\left\{B^{\prime} \in \mathcal{B} ; \operatorname{pos}\left(B^{\prime}, F\left(B^{\prime}\right)\right)=w\right\}
$$

via $B^{\prime} \leftrightarrow\left(B^{\prime}, F\left(B^{\prime}\right)\right.$. Note that $\mathcal{B}_{w}$ is stable under conjugation by $\Gamma$. For $\mathcal{L} \in$ $\mathcal{S}(T)^{w F}$, the local system $\mathcal{L}_{(w)}$ on $Z^{\mathbf{w}}$ can be then identified with a local system $\mathcal{L}_{w}$ on $\mathcal{B}_{w}$. The subvarieties $\mathcal{B}_{w}$ of $\mathcal{B}$ and the local systems $\mathcal{L}_{w}$ were introduced in DL.

2.6. In the remainder of this section we fix a sequence $\mathbf{s}=\left(s_{1}, s_{2}, \ldots, s_{r}\right)$ in $\mathbf{I}$ and $\mathcal{L} \in \mathcal{S}(T)^{[\mathbf{s}] F}$. Let $\mathcal{I}_{\mathbf{s}}$ be as in 2.4. Let

$$
\begin{gathered}
\bar{Z}^{\mathbf{s}}=\left\{\left(B_{i}\right) \in \mathcal{B}^{[0, r]} ; \operatorname{pos}\left(B_{i-1}, B_{i}\right) \in\left\{1, s_{i}\right\}(i \in[1, r]), B_{r}=F\left(B_{0}\right)\right\}, \\
\mathcal{Z}^{\mathbf{s}}=\left\{\left(B_{i}\right) \in \mathcal{B}^{[0, r]} ; \operatorname{pos}\left(B_{i-1}, B_{i}\right) \in\left\{1, s_{i}\right\}\left(i \in \mathcal{I}_{\mathbf{s}}\right)\right. \\
\left.\operatorname{pos}\left(B_{i-1}, B_{i}\right)=s_{i}\left(i \in[1, r]-\mathcal{I}_{\mathbf{s}}\right), B_{r}=F\left(B_{0}\right)\right\} .
\end{gathered}
$$

The variety $\bar{Z}^{\mathbf{s}}$ was introduced in $\left[\mathrm{DL}\right.$ (in the case where $l([\mathbf{s}])=r$ ). For $\mathcal{J} \subset \mathcal{I}_{\mathbf{s}}$ the variety $Z^{\mathbf{s} \mathcal{J}}$ (as in 2.5) can be also described as

$$
\begin{gathered}
Z^{\mathbf{s} \mathcal{J}}=\left\{\left(B_{i}\right) \in \mathcal{B}^{[0, r]} ; B_{i-1}=B_{i}(i \in \mathcal{J}), \operatorname{pos}\left(B_{i-1}, B_{i}\right)=s_{i}(i \in[1, r]-\mathcal{J}),\right. \\
\left.B_{r}=F\left(B_{0}\right)\right\} \subset \mathcal{Z}^{\mathbf{s}} .
\end{gathered}
$$

Consider the commutative diagram

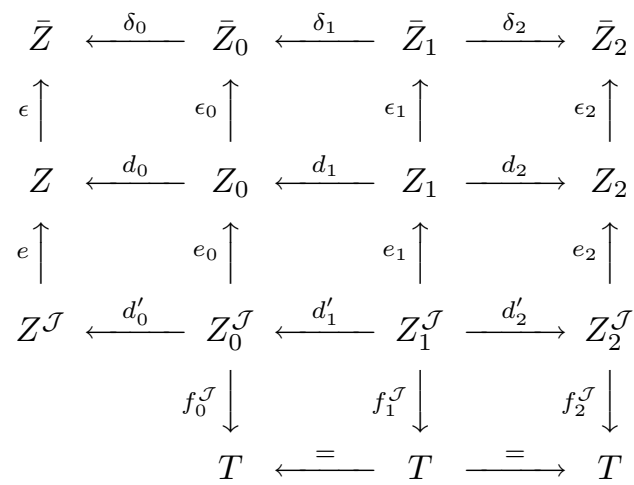

where the following notation is used.

$\bar{Z}=\bar{Z}^{\mathbf{s}}, Z=\mathcal{Z}^{\mathrm{s}}, Z^{\mathcal{J}}=Z^{\mathbf{s}_{\mathcal{J}}}$.

$\bar{Z}_{0}$ is the set of all $\left(g_{0} U, g_{1} U, \ldots, g_{r} U\right) \in(G / U)^{[0, r]}$ such that $g_{i-1}^{-1} g_{i} \in P_{s_{i}}$ for $i \in[1, r]$ and $g_{r}^{-1} F\left(g_{0}\right) \in U$.

$Z_{0}$ is the set of all $\left(g_{0} U, g_{1} U, \ldots, g_{r} U\right) \in(G / U)^{[0, r]}$ such that $g_{i-1}^{-1} g_{i} \in P_{s_{i}}$ for $i \in \mathcal{I}_{\mathbf{s}}, g_{i-1}^{-1} g_{i} \in P_{s_{i}}-B$ for $i \in[1, r]-\mathcal{I}_{\mathbf{s}}$ and $g_{r}^{-1} F\left(g_{0}\right) \in U$. 
$Z_{0}^{\mathcal{J}}$ is the set of all $\left(g_{0} U, g_{1} U, \ldots, g_{r} U\right) \in(G / U)^{[0, r]}$ such that $g_{i-1}^{-1} g_{i} \in P_{s_{i}}-B$ for $i \in[1, r]-\mathcal{J}, g_{i-1}^{-1} g_{i} \in B$ for $i \in \mathcal{J}$ and $g_{r}^{-1} F\left(g_{0}\right) \in U$.

$d_{0}, \delta_{0}$ are given by $\left(g_{0} U, g_{1} U, \ldots, g_{r} U\right) \mapsto\left(g_{0} B g_{0}^{-1}, g_{1} B g_{1}^{-1}, \ldots, g_{r} B g_{r}^{-1}\right)$.

$f_{0}^{\mathcal{J}}$ is given by $\left(g_{0} U, g_{1} U, \ldots, g_{r} U\right) \mapsto k\left(g_{0}^{-1} g_{1}\right) \ldots k\left(g_{r-1}^{-1} g_{r}\right)\left[\mathbf{s}_{\mathcal{J}}\right]^{\bullet-1}$.

$\bar{Z}_{1}$ is the set of all $\left(y_{0}, y_{1}, \ldots, y_{r}\right) \in G^{[0, r]}$ such that $y_{i} \in P_{s_{i}}(i \in[1, r])$, $y_{0}^{-1} F\left(y_{0}\right) \in y_{1} y_{2} \ldots y_{r} U$.

$Z_{1}$ is the set of all $\left(y_{0}, y_{1}, \ldots, y_{r}\right) \in G^{[0, r]}$ such that $y_{i} \in P_{s_{i}}\left(i \in \mathcal{I}_{\mathbf{s}}\right), y_{i} \in$ $P_{s_{i}}-B\left(i \in[1, r]-\mathcal{I}_{\mathbf{s}}\right), y_{0}^{-1} F\left(y_{0}\right) \in y_{1} y_{2} \ldots y_{r} U$.

$Z_{1}^{\mathcal{J}}$ is the set of all $\left(y_{0}, y_{1}, \ldots, y_{r}\right) \in G^{[0, r]}$ such that $y_{i} \in P_{s_{i}}-B(i \in[1, r]-\mathcal{J})$, $y_{i} \in B(i \in \mathcal{J}), y_{0}^{-1} F\left(y_{0}\right) \in y_{1} y_{2} \ldots y_{r} U$.

$d_{1}, \delta_{1}$ are given by $\left(y_{0}, y_{1}, \ldots, y_{r}\right) \mapsto\left(y_{0} U, y_{0} y_{1} U, \ldots, y_{0} y_{1} \ldots y_{r} U\right)$.

$f_{1}^{\mathcal{J}}$ is $\left(y_{0}, y_{1}, \ldots, y_{r}\right) \mapsto k\left(y_{1}\right) k\left(y_{2}\right) \ldots k\left(y_{r}\right)\left[\mathbf{s}_{\mathcal{J}}\right]^{\bullet-1}$.

$\bar{Z}_{2}=Y, Z_{2}=\mathcal{Y}, Z_{2}^{\mathcal{J}}=\mathcal{Y}^{\mathcal{J}}$. (See 2.4.)

$d_{2}, \delta_{2}$ are given by $\left(y_{0}, y_{1}, \ldots, y_{r}\right) \mapsto\left(y_{1}, \ldots, y_{r}\right)$.

$f_{2}^{\mathcal{J}}$ is $\left(y_{1}, \ldots, y_{r}\right) \mapsto k\left(y_{1}\right) k\left(y_{2}\right) \ldots k\left(y_{r}\right)\left[\mathbf{s}_{\mathcal{J}}\right]^{\bullet-1}$.

The maps $e, e_{i}, \epsilon_{i}(i \in[0,2])$ are the obvious imbeddings. For $i \in[0,2]$ the map $d_{i}^{\prime}$ is the restriction of $d_{i}$. From the definitions we have:

(a) In our commutative diagram, all squares that do not involve $T$ are cartesian.

2.7. $\Gamma$ acts:

on $Z$ and $\bar{Z}$ by $g:\left(B_{0}, B_{1}, \ldots, B_{r}\right) \mapsto\left(g B_{0} g^{-1}, g B_{1} g^{-1}, \ldots, g B_{r} g^{-1}\right)$;

on $Z_{0}$ and $\bar{Z}_{0}$ by $g:\left(g_{0} U, g_{1} U, \ldots, g_{r} U\right) \mapsto\left(g g_{0} U, g g_{1} U, \ldots, g g_{r} U\right)$;

on $Z_{1}$ and $\bar{Z}_{1}$ by $g:\left(y_{0}, y_{1}, \ldots, y_{r}\right) \mapsto\left(g y_{0}, y_{1}, y_{2}, \ldots, y_{r}\right)$;

on $Z_{2}, \bar{Z}_{2}$ trivially.

The subsets $Z^{\mathcal{J}}, Z_{i}^{\mathcal{J}}$ of $Z, Z_{i}(i \in[0,2])$ are stable under the $\Gamma$-action. The maps $d_{i}, d_{i}^{\prime}, \delta_{i}, f_{i}^{\mathcal{J}}$ are compatible with the $\Gamma$-actions.

$\underline{B}$ (see 2.4) acts:

on $Z$ trivially;

on $Z_{0}$ by $\left(b_{0}, b_{1}, \ldots, b_{r}\right):\left(g_{0} U, g_{1} U, \ldots, g_{r} U\right) \mapsto\left(g_{0} b_{0}^{-1} U, g_{1} b_{1}^{-1} U, \ldots, g_{r} b_{r}^{-1} U\right)$;

on $Z_{1}$ by $\left(b_{0}, b_{1}, \ldots, b_{r}\right):\left(y_{0}, y_{1}, \ldots, y_{r}\right) \mapsto\left(y_{0} b_{0}^{-1}, b_{0} y_{1} b_{1}^{-1}, b_{1} y_{2} b_{2}^{-1}, \ldots, b_{r-1} y_{r} b_{r}^{-1}\right)$; on $Z_{2}$ by $\left(b_{0}, b_{1}, \ldots, b_{r}\right):\left(y_{1}, \ldots, y_{r}\right) \mapsto\left(b_{0} y_{1} b_{1}^{-1}, b_{1} y_{2} b_{2}^{-1}, \ldots, b_{r-1} y_{r} b_{r}^{-1}\right)$.

The maps $d_{i}$ are compatible with the $\underline{B}$-actions.

\subsection{Now}

(a) $d_{0}, \delta_{0}, d_{0}^{\prime}$ are principal $\underline{B} / U^{[0, r]}$-bundles.

(b) $d_{1}, \delta_{1}, d_{1}^{\prime}$ are principal $U^{[0, r]}$-bundles. (The action of $U^{[0, r]}$ on $\bar{Z}_{1}, Z_{1}, Z_{1}^{\mathcal{J}}$ is by restriction of the $\underline{B}$-action.)

(c) Each of $d_{2}, \delta_{2}, d_{2}^{\prime}$ is a composition of a principal $\Gamma$-bundle with a principal $U$-bundle.

2.9. We show for $i \in[0,2]$ that

(a) $Z_{i}$ is smooth of pure dimension say $\mathfrak{d}_{i}$ and it is open dense in $\bar{Z}_{i}$.

Let $P_{i}$ be the property expressed by (a). It is obvious that $P_{2}$ holds. Using $P_{2}$, 2.6(a) and 2.8(c) we see that $P_{1}$ holds. Using $P_{1}, 2.6(\mathrm{a})$ and $2.8(\mathrm{~b})$ we see that $P_{0}$

holds. Thus (a) holds. Using $P_{0}, 2.6(\mathrm{a})$ and $2.8(\mathrm{a})$ we see that

(b) $Z$ is smooth of pure dimension say $\mathfrak{d}$ and it is open dense in $\bar{Z}$.

We show:

(c) $\mathfrak{d}=r$. 
From the definitions we see that $\mathfrak{d}_{2}=r(\operatorname{dim} B+1)$. From the arguments above we see successively that $\mathfrak{d}_{1}=r(\operatorname{dim} B+1)+\operatorname{dim} U, \mathfrak{d}_{0}=\mathfrak{d}_{1}-(r+1) \operatorname{dim} U$ $\mathfrak{d}=\mathfrak{d}_{0}-r \operatorname{dim} T ;(\mathrm{c})$ follows.

We show:

(d) The natural $\Gamma$-action on the set of connected components of $Z_{i}(i \in[0,2])$ or of $Z$ is transitive.

For $Z_{2}$ this is clear since $Z_{2}$ is connected. This also implies the result for $Z_{1}$ (see 2.8(c)). Using 2.8(b),(a) we deduce that the result also holds for $Z_{0}$ and for $Z$.

2.10. Let $\mathcal{J} \subset \mathcal{I}_{\mathbf{s}}$. For $i \in[0,2]$ we set $\mathcal{L}_{i}^{\mathcal{J}}=\left(f_{i}^{\mathcal{J}}\right)^{*} \mathcal{L}$, a local system of rank 1 on $Z_{i}^{\mathcal{J}}$. Since $\mathcal{L}$ has a natural $\Gamma$-equivariant structure (with $\Gamma$ acting trivially on each stalk) and $f_{i}^{\mathcal{J}}$ is compatible with the $\Gamma$-actions we see that $\mathcal{L}_{i}^{\mathcal{J}}$ has a natural $\Gamma$-equivariant structure. From the definitions we have isomorphisms compatible with the $\Gamma$-equivariant structures as follows:

$$
d_{1}^{\prime *} \mathcal{L}_{1}^{\mathcal{J}} \cong \mathcal{L}_{0}^{\mathcal{J}} ; d_{2}^{\prime *} \mathcal{L}_{2}^{\mathcal{J}} \cong \mathcal{L}_{1}^{\mathcal{J}}
$$

From the definitions we see that

(b) $\Gamma$ acts trivially on any stalk of $\mathcal{L}_{2}^{\mathcal{J}}$.

Let $\mathcal{L}^{\mathcal{J}}$ be the local system on $Z^{\mathcal{J}}=Z^{\mathbf{s} \mathcal{J}}$ denoted in 2.5 by $\mathcal{L}_{\mathbf{w}}$ where $\mathbf{w}=\mathbf{s}_{\mathcal{J}}$. This is well defined since for $\mathcal{J} \subset \mathcal{I}_{\mathbf{s}}$ we have $\mathcal{L} \in \mathcal{S}(T)^{\left[\mathbf{s}_{\mathcal{J}}\right] F}$. (We use that $\mathcal{L} \in \mathcal{S}(T)^{[\mathbf{s}] F}$ and $\left(s_{1} s_{2} \ldots s_{j} \ldots s_{2} s_{1}\right)^{*} \mathcal{L} \cong \mathcal{L}$ for any $j \in \mathcal{J}$.) As in $2.5, \mathcal{L}^{\mathcal{J}}$ has a natural $\Gamma$-equivariant structure. From the definitions we have $d_{0}^{\prime *} \mathcal{L}^{\mathcal{J}} \cong \mathcal{L}_{0}^{\mathcal{J}}$ compatibly with the $\Gamma$-equivariant structures.

2.11. For $i \in[1,2]$ we define a local system $\overline{\mathcal{L}}_{i}$ on $Z_{i}$ by $\overline{\mathcal{L}}_{2}=\underline{\underline{\mathcal{L}}}, \overline{\mathcal{L}}_{1}=d_{2}^{*} \overline{\mathcal{L}}_{2}$ where $\underline{\mathcal{L}}$ is as in 2.4. From 2.4(b) and the results in 2.7 we see that $\overline{\mathcal{L}}_{i}$ is $\underline{B}$-equivariant. Since $d_{0} d_{1}: Z_{1} \rightarrow Z$ is a principal $\underline{B}$-bundle, we see that there is a well defined local system $\overline{\mathcal{L}}$ on $Z$ such that $\left(d_{0} d_{1}\right)^{*} \overline{\mathcal{L}}=\overline{\mathcal{L}}_{1}$. Let $\overline{\mathcal{L}}_{0}=d_{0}^{*} \overline{\mathcal{L}}$. Then $\overline{\mathcal{L}}_{1}=d_{1}^{*} \overline{\mathcal{L}}_{0}$. We regard $\overline{\mathcal{L}}_{2}$ as a $\Gamma$-equivariant local system on $Z_{2}$ with $\Gamma$ acting trivially on each stalk. Since each $d_{i}$ is compatible with the $\Gamma$-actions we see that $\overline{\mathcal{L}}_{i}(i \in[0,2])$ and $\overline{\mathcal{L}}$ have natural $\Gamma$-equivariant structures which are compatible with $d_{i}^{*}$.

2.12. We show:

(a) For any $\mathcal{J} \subset \mathcal{I}_{\text {s }}$ we have $\left.\overline{\mathcal{L}}\right|_{Z^{\mathcal{J}}} \cong \mathcal{L}^{\mathcal{J}}$ compatibly with the $\Gamma$-equivariant structures.

(b) For any $i \in[0,2]$ and $\mathcal{J} \subset \mathcal{I}_{\mathbf{s}}$ we have $\left.\overline{\mathcal{L}}_{i}\right|_{Z_{i}^{\mathcal{J}}} \cong \mathcal{L}_{i}^{\mathcal{J}}$ compatibly with the $\Gamma$-equivariant structures.

Note that (b) holds for $i=2$ by 2.4 (a) (the compatibility with the $\Gamma$-equivariant structures is automatic since $\Gamma$ acts trivially on each stalk of the local systems involved). From this we get (using $2.10,2.11$ ) that (b) holds for $i=1$, then for $i=0$, and then that (a) holds.

\subsection{We show:}

(a) We have $\left.\operatorname{IC}(\bar{Z}, \overline{\mathcal{L}})\right|_{\bar{Z}-Z}=0$.

(b) For $i \in[0,2]$ we have $\left.\operatorname{IC}\left(\bar{Z}_{i}, \overline{\mathcal{L}}_{i}\right)\right|_{\bar{Z}_{i}-Z_{i}}=0$.

Note that the $I C$ complexes in (a),(b) are well defined by 2.9(a),(b). Now (b) holds for $i=2$ by 2.4 (b). From this we get (using $2.8,2.11$ ) that (b) holds for $i=1$, then for $i=0$, and then that (a) holds. 
2.14. Assume that $r \geq 2, h \in[2, r] \cap \mathcal{I}_{\mathbf{s}}, s_{h-1}=s_{h}$. We set

$$
\mathbf{s}^{\prime}:=\left(s_{1}, \ldots, s_{h-1}, s_{h+1}, \ldots, s_{r}\right) .
$$

Then $\mathcal{L} \in \mathcal{S}(T)^{\left[\mathbf{s}^{\prime}\right] F}$ so that $Z^{\prime}:=\mathcal{Z}^{\mathbf{s}^{\prime}}$ is defined as in 2.6. We have a commutative diagram

where:

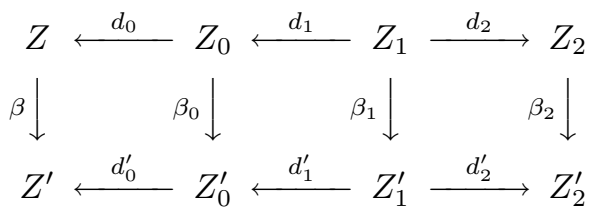

the upper row is as in 2.6,

the lower row is defined analogously in terms of $\mathbf{s}^{\prime}, \mathcal{L}$ instead of $\mathbf{s}, \mathcal{L}$,

$\beta$ is $\left(B_{0}, B_{1}, \ldots, B_{r}\right) \mapsto\left(B_{0}, B_{1}, \ldots, B_{h-2}, B_{h}, \ldots, B_{r}\right)$,

$\beta_{0}$ is $\left(g_{0} U, g_{1} U, \ldots, g_{r} U\right) \mapsto\left(g_{0} U, g_{1} U, \ldots, g_{h-2} U, g_{h} U, g_{r} U\right)$,

$\beta_{1}$ is $\left(y_{0}, y_{1}, \ldots, y_{r}\right) \mapsto\left(y_{0}, y_{1}, \ldots, y_{h-2}, y_{h-1} y_{h}, y_{h+1}, \ldots, y_{r}\right)$,

$\beta_{2}$ is $\left(y_{1}, \ldots, y_{r}\right) \mapsto\left(y_{1}, \ldots, y_{h-2}, y_{h-1} y_{h}, y_{h+1}, \ldots, y_{r}\right)$.

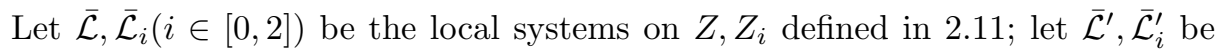
the analogous local systems on $Z^{\prime}, Z_{i}^{\prime}$. We show:

(a)

$$
\overline{\mathcal{L}} \cong \beta^{*}\left(\overline{\mathcal{L}}^{\prime}\right) .
$$

It is enough to show that $\delta_{1}^{*} \delta_{0}^{*} \overline{\mathcal{L}} \cong \delta_{1}^{*} \delta_{0}^{*} \beta^{*}\left(\overline{\mathcal{L}}^{\prime}\right)$ or equivalently that $\overline{\mathcal{L}}_{1} \cong \beta_{1}^{*} \overline{\mathcal{L}}_{1}^{\prime}$. Hence it is enough to show that $\delta_{2}^{*} \overline{\mathcal{L}}_{2} \cong \beta_{1}^{*} \delta_{2}^{\prime *} \overline{\mathcal{L}}_{2}^{\prime}$ or equivalently that $\delta_{2}^{*} \overline{\mathcal{L}}_{2} \cong$ $\delta_{2}^{*} \beta_{2}{ }^{*} \overline{\mathcal{L}}_{2}^{\prime}$. It is enough to show that $\overline{\mathcal{L}}_{2} \cong \beta_{2}{ }^{*} \overline{\mathcal{L}}_{2}^{\prime}$. From the definition of $\mathcal{L}$ in 2.4 and with the notation in 2.4 we see that it is enough to show that $m^{*} \underline{\overline{\mathcal{L}}} \cong$ ${ }^{\prime} \underline{\mathcal{L}} \nabla^{\prime} \underline{\mathcal{L}}$ (local systems on $P_{s_{h}}$ ) where ' $\mathcal{L}=s_{h-1}^{*} \ldots s_{2}^{*} s_{1}^{*} \mathcal{L} \cong s_{h}^{*} s_{h-1}^{*} \ldots s_{2}^{*} s_{1}^{*} \mathcal{L}$ and $m: P_{s_{h}} \times P_{s_{h}} \rightarrow P_{s_{h}}$ is multiplication. It is enough to show that, in the setup of 2.1 we have $m^{\prime *} \underline{\mathcal{L}} \cong \mathcal{L} \unrhd \underline{\mathcal{L}}$ (local systems on $G$ ) where $m^{\prime}: G \times G \rightarrow G$ is multiplication. This follows from the definitions in 2.1 using the isomorphism $m_{1}^{*} \mathcal{L} \cong \mathcal{L} \otimes \mathcal{L}$ (local systems on $T$ ) where $m_{1}: T \times T \rightarrow T$ is multiplication.

2.15. Assume that $r \geq 2, h \in[2, r], h \notin \mathcal{I}_{\mathbf{s}}, s_{h-1}=s_{h}$. Let $Z^{1}$ be the open subset of $Z$ defined by the condition $\operatorname{pos}\left(B_{h-2}, B_{h}\right)=s_{i}$. Define $\beta: Z^{1} \rightarrow \mathcal{B}^{r}$ by $\left(B_{0}, B_{1}, \ldots, B_{r}\right) \mapsto\left(B_{0}, B_{1}, \ldots, B_{h-2}, B_{h}, \ldots, B_{r}\right)$. We show:

$$
\beta_{!}\left(\left.\overline{\mathcal{L}}\right|_{Z^{1}}\right)=0 \text {. }
$$

Let $p=\left(B_{0}, B_{1}, \ldots, B_{h-2}, B_{h}, \ldots, B_{r}\right) \in \mathcal{B}^{r}$ be such that $\Phi:=\beta^{-1}(p) \neq \emptyset$. Then $\Phi=\left\{\left(B_{0}, B_{1}, \ldots, B_{h-2}, \tilde{B}, B_{h}, \ldots, B_{r}\right) ; \tilde{B} \in \mathcal{B}, \operatorname{pos}\left(B_{h-2}, \tilde{B}\right)=\operatorname{pos}\left(\tilde{B}, B_{h}\right)=s_{h}\right\}$.

It is enough to show that $H_{c}^{*}(\Phi, \overline{\mathcal{L}})=0$. Let

$$
\begin{aligned}
\Phi^{\prime}= & \left\{\left(B_{0}, B_{1}, \ldots, B_{h-2}, \tilde{B}, B_{h}, \ldots, B_{r}\right) ; \tilde{B} \in \mathcal{B}, \operatorname{pos}\left(B_{h-2}, \tilde{B}\right)=s_{h},\right. \\
& \left.\operatorname{pos}\left(\tilde{B}, B_{h}\right) \in\left\{1, s_{h}\right\}\right\} .
\end{aligned}
$$

Then $\Phi^{\prime}$ is an affine line which is a cross section in $\bar{Z}$ to the divisor $\Delta_{h}$ (see 2.4) and $\Phi^{\prime} \cap \Delta_{h}$ is the point $p^{\prime}=\left(B_{0}, B_{1}, \ldots, B_{h-2}, B_{h}, B_{h}, \ldots, B_{r}\right)$. Moreover, $\Phi=\Phi^{\prime}-\left\{p^{\prime}\right\}$. The vanishing $H_{c}^{*}(\Phi, \overline{\mathcal{L}})=0$ follows from $2.4(\mathrm{c})$.

Now let $Z^{2}=Z-Z^{1}$, that is the closed subset of $Z$ defined by the condition $B_{h-2}=B_{h}$. Let $\mathbf{s}^{\prime}:=\left(s_{1}, \ldots, s_{h-2}, s_{h+1}, \ldots, s_{r}\right)$. We have $\mathcal{L} \in \mathcal{S}(T)^{\left[\mathbf{s}^{\prime}\right] F}$ so that $Z^{\prime}:=\mathcal{Z}^{\mathbf{s}^{\prime}}$ is defined as in 2.6. Define $\beta^{\prime}: Z^{2} \rightarrow Z^{\prime}$ by $\left(B_{0}, B_{1}, \ldots, B_{r}\right) \mapsto$ 
$\left(B_{0}, B_{1}, \ldots, B_{h-2}, B_{h+1}, \ldots, B_{r}\right)$, an affine line bundle. Let $\overline{\mathcal{L}}$ be the local systems on $Z$ defined in 2.11 ; let $\overline{\mathcal{L}}^{\prime}$ be the analogous local system on $Z^{\prime}$. From the definitions we have

$$
\left.\overline{\mathcal{L}}\right|_{Z^{2}}=\beta^{*}\left(\overline{\mathcal{L}}^{\prime}\right)
$$

\section{The Class $\mathbb{S}^{\prime}\left(\mathcal{P}_{J}\right)$ of Simple obJeCts in $\mathcal{M}_{\Gamma}\left(\mathcal{P}_{J}\right)$}

3.1. Let $J \subset \mathbf{I}$. We view $\mathcal{P}_{J}$ as a variety with $\Gamma$-action (conjugation). Hence $\mathcal{M}_{\Gamma}\left(\mathcal{P}_{J}\right)$ is well defined.

3.2. Let $\mathcal{L} \in \mathcal{S}(T)$. If $\mathbf{w}$ is as in 2.5 and $\mathcal{L} \in \mathcal{S}(T)^{[\mathbf{w}] F}$, then the local system $\mathcal{L}_{\mathbf{w}}$ on $Z^{\mathbf{w}}$ has a natural $\Gamma$-equivariant structure (see 2.5). The map $\Pi^{\mathbf{w}}: Z^{\mathbf{w}} \rightarrow \mathcal{P}_{J}$, $\left(B_{0}, B_{1}, \ldots, B_{r}\right) \mapsto P_{B_{0}, J}$, commutes with the $\Gamma$-actions. Hence for any $j \in \mathbf{Z}$, ${ }^{p} H^{j}\left(\Pi_{!}^{\mathbf{w}} \mathcal{L}_{\mathbf{w}}\right)$ is an object of $\mathcal{M}_{\Gamma}\left(\mathcal{P}_{J}\right)$.

If $\mathbf{s}$ is as in 2.6 and $\mathcal{L} \in \mathcal{S}(T)^{[\mathbf{s}] F}$, then the local system $\overline{\mathcal{L}}$ on $\mathcal{Z}^{\mathbf{s}}$ has a natural $\Gamma$-equivariant structure (see 2.6, 2.11). Hence $\overline{\mathcal{L}}^{\sharp}=I C\left(\bar{Z}^{\mathbf{s}}, \overline{\mathcal{L}}\right)$ (see 2.6, 2.11, 2.13) has a natural $\Gamma$-equivariant structure. Define $\Upsilon^{\mathbf{s}}: \mathcal{Z}^{\mathbf{s}} \rightarrow \mathcal{P}_{J}$ and $\bar{\Upsilon}^{\mathbf{s}}: \bar{Z}^{\mathbf{s}} \rightarrow \mathcal{P}_{J}$ by $\left(B_{0}, B_{1}, \ldots, B_{r}\right) \mapsto P_{B_{0}, J}$. These maps commute with the $\Gamma$-actions. Hence for any $j \in \mathbf{Z}$,

$$
{ }^{p} H^{j}\left(\Upsilon_{!}^{\mathbf{s}} \overline{\mathcal{L}}\right)={ }^{p} H^{j}\left(\bar{\Upsilon}_{!}^{\mathbf{s}} \overline{\mathcal{L}}^{\sharp}\right)
$$

is an object of $\mathcal{M}_{\Gamma}\left(\mathcal{P}_{J}\right)$. (The equality in (a) follows from by 2.13.)

In 3.3-3.7 we will show that the following conditions for a simple object $K$ in $\mathcal{M}_{\Gamma}\left(\mathcal{P}_{J}\right)$ are equivalent:

(i) $K \dashv_{\Gamma}{ }^{p} H^{\cdot}\left(\Pi_{!}^{\mathbf{w}} \mathcal{L}_{\mathbf{w}}\right)$ for some one term sequence $\mathbf{w}$ in $W$ such that $\mathcal{L} \in$ $\mathcal{S}(T)^{[\mathbf{w}] F}$.

(ii) $K \dashv_{\Gamma}{ }^{p} H^{\cdot}\left(\Pi_{!}^{\mathbf{w}} \mathcal{L}_{\mathbf{w}}\right)$ for some sequence $\mathbf{w}$ in $W$ such that $\mathcal{L} \in \mathcal{S}(T)^{[\mathbf{w}] F}$.

(iii) $K \dashv_{\Gamma}^{p} H^{\cdot}\left(\Pi_{!}^{\mathbf{w}} \mathcal{L}_{\mathbf{w}}\right)$ for some sequence $\mathbf{w}$ in $\mathbf{I} \cup\{1\}$ such that $\mathcal{L} \in \mathcal{S}(T)^{[\mathbf{w}] F}$.

(iv) $K \dashv_{\Gamma}{ }^{p} H^{\cdot}\left(\Pi_{!}^{\mathbf{s}} \mathcal{L}_{\mathbf{s}}\right)$ for some sequence $\mathbf{s}$ in $\mathbf{I}$ such that $\mathcal{L} \in \mathcal{S}(T)^{[\mathbf{s}] F}$.

(v) $K \dashv_{\Gamma}^{p} H^{\cdot}\left(\Upsilon_{!}^{\mathbf{s}} \overline{\mathcal{L}}\right)$ for some sequence $\mathbf{s}$ in $\mathbf{I}$ such that $\mathcal{L} \in \mathcal{S}(T)^{[\mathbf{s}] F}$.

(vi) $K \dashv_{\Gamma}^{p} H^{\cdot}\left(\bar{\Upsilon}_{!}^{\mathbf{s}} \overline{\mathcal{L}}^{\sharp}\right)$ for some sequence $\mathbf{s}$ in $\mathbf{I}$ such that $\mathcal{L} \in \mathcal{S}(T)^{[\mathbf{s}] F}$.

3.3. Let $\mathbf{w}=\left(w_{1}, \ldots, w_{r}\right)$ be a sequence in $W$ such that $\mathcal{L} \in \mathcal{S}(T)^{[\mathbf{w}] F}$. Assume that for some $i \in[1, r], w_{i}^{\prime}, w_{i}^{\prime \prime} \in W$ satisfy $w_{i}=w_{i}^{\prime} w_{i}^{\prime \prime}$ and $l\left(w_{i}\right)=l\left(w_{i}^{\prime}\right)+l\left(w_{i}^{\prime \prime}\right)$. Let $\mathbf{w}^{\prime}=\left(w_{1}, \ldots, w_{i-1}, w_{i}^{\prime}, w_{i}^{\prime \prime}, w_{i+1}, \ldots, w_{r}\right)$. Define an isomorphism $Z^{\mathbf{w}^{\prime}} \stackrel{\sim}{\rightarrow} Z^{\mathbf{w}}$ by

$$
\left(B_{0}, B_{1}, \ldots, B_{r+1}\right) \mapsto\left(B_{0}, B_{1}, \ldots, B_{i-1}, B_{i+1}, \ldots, B_{r+1}\right) .
$$

This isomorphism is compatible with the $\Gamma$-actions, with the maps $\Pi^{\mathbf{w}^{\prime}}, \Pi^{\mathbf{w}}$ and with the local systems $\mathcal{L}_{\mathbf{w}^{\prime}}, \mathcal{L}_{\mathbf{w}}$. Hence for any $j$ we have

$$
{ }^{p} H^{j}\left(\Pi_{!}^{\mathbf{w}} \mathcal{L}_{\mathbf{w}}\right)={ }^{p} H^{j}\left(\Pi_{!}^{\mathbf{w}^{\prime}} \mathcal{L}_{\mathbf{w}^{\prime}}\right)
$$

(as objects of $\mathcal{M}_{\Gamma}\left(\mathcal{P}_{J}\right)$ ). Applying (a) repeatedly we see that conditions 3.2(ii), 3.2 (iii), 3.2(iv) are equivalent. 
3.4. We prove the equivalence of conditions $3.2(\mathrm{iii}), 3.2(\mathrm{v})$.

Let $\mathbf{s}=\left(s_{1}, \ldots, s_{r}\right)$ be a sequence in $\mathbf{I}$ such that $\mathcal{L} \in \mathcal{S}(T)^{[\mathbf{s}] F}$. Define a sequence ${ }^{0} \mathcal{Z} \supset{ }^{1} \mathcal{Z} \supset \ldots$ of closed subsets of $\mathcal{Z}^{\mathbf{s}}$ by ${ }^{i} \mathcal{Z}=\cup_{\mathcal{J} \subset \mathcal{I}_{\mathbf{s}} ;|\mathcal{J}| \geq i} Z^{\mathbf{s}_{\mathcal{J}}}$ (notation of 2.6). Let $f^{i}:{ }^{i} \mathcal{Z} \rightarrow \mathcal{Z}^{\mathbf{s}}, f^{\prime i}:{ }^{i} \mathcal{Z}-{ }^{i+1} \mathcal{Z} \rightarrow \mathcal{Z}^{\mathbf{s}}$ be the inclusions. The natural distinguished triangle

$$
\left(\Upsilon_{!}^{\mathbf{s}} f_{!}^{\prime i} f^{\prime i *} \overline{\mathcal{L}}, \Upsilon_{!}^{\mathbf{s}} f_{!}^{i} f^{i *} \overline{\mathcal{L}}, \Upsilon_{!}^{\mathbf{s}} f_{!}^{i+1}\left(f^{i+1 *} \overline{\mathcal{L}}\right)\right)
$$

gives rise for any $i \geq 0$ to a long exact sequence in $\mathcal{M}_{\Gamma}\left(\mathcal{P}_{J}\right)$ :

$$
\cdots \rightarrow{ }^{p} H^{j-1}\left(\Upsilon_{!}^{\mathbf{s}} f_{!}^{i+1} f^{i+1 *} \overline{\mathcal{L}}\right) \rightarrow \oplus_{\mathcal{J} \subset \mathcal{I}_{\mathbf{s}} ;|\mathcal{J}|=i}{ }^{p} H^{j}\left(\Pi_{!}^{\mathbf{s} \mathcal{J}} \mathcal{L}_{\mathbf{s}_{\mathcal{J}}}\right) \rightarrow{ }^{p} H^{j}\left(\Upsilon_{!}^{\mathbf{s}} f_{!}^{i} f^{i *} \overline{\mathcal{L}}\right)
$$

(a)

$$
\rightarrow{ }^{p} H^{j}\left(\Upsilon_{!}^{\mathbf{s}} f_{!}^{i+1} f^{i+1 *} \overline{\mathcal{L}}\right) \rightarrow \oplus_{\mathcal{J} \subset \mathcal{I}_{\mathbf{s}} ;|\mathcal{J}|=i}^{p} H^{j+1}\left(\Pi_{!}^{\mathbf{s}}{ }^{\mathcal{J}} \mathcal{L}_{\mathbf{s}_{\mathcal{J}}}\right) \rightarrow \ldots
$$

Here we have used the equality

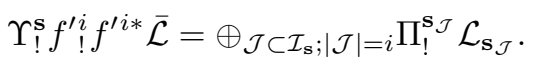

(See 2.12(a).) Note that $\Upsilon_{!}^{\mathbf{s}} f_{!}^{0} f^{0 *} \overline{\mathcal{L}}=\Upsilon_{!}^{\mathbf{s}} \overline{\mathcal{L}}$ and $\Upsilon_{!}^{\mathbf{s}} f_{!}^{i} f^{i *} \overline{\mathcal{L}}=0$ for $i$ large.

If $K$ does not satisfy 3.2(iii), then from (b) we see that for any $i \geq 0$ we have $K \dashv_{\Gamma}$ ${ }^{p} H^{\cdot}\left(\Upsilon_{!}^{\mathbf{s}} f_{!}^{i} f^{i *} \overline{\mathcal{L}}\right)$ if and only if $K \dashv_{\Gamma}{ }^{p} H^{\cdot}\left(\Upsilon_{!}^{\mathbf{s}} f_{!}^{i+1} f^{i+1 *} \overline{\mathcal{L}}\right)$. Since $K \bigwedge_{\Gamma}{ }^{p} H^{\cdot}\left(\Upsilon_{!}^{\mathbf{s}} f_{!}^{i} f^{i *} \overline{\mathcal{L}}\right)$ with large $i$, it follows that $K \rtimes_{\Gamma}^{p} H^{\cdot}\left(\Upsilon_{!}^{\mathbf{s}} f_{!}^{0} f^{0 *} \overline{\mathcal{L}}\right)$; that is, $K \AA_{\Gamma}{ }^{p} H^{\cdot}\left(\Upsilon_{!}^{\mathbf{s}} \overrightarrow{\mathcal{L}}\right)$. Thus, $K$ does not satisfy $3.2(\mathrm{v})$.

Assume now that $K$ satisfies 3.2(iii). We may assume that $K \dashv_{\Gamma}{ }^{p} H^{\cdot}\left(\Pi_{!}^{\mathbf{s}} \mathcal{L}_{\mathbf{s}}\right)$ where $\mathbf{s}$ as in 3.2(iii) has a minimum possible number of terms in $\mathbf{I}$. By the equivalence of 3.2(ii), 3.2(iii) we see that we may assume that all terms of $\mathbf{s}$ are in $\mathbf{I}$ and that $K \AA_{\Gamma}{ }^{p} H^{\cdot}\left(\Pi_{!}^{\mathbf{s}} \mathcal{L}_{\mathbf{s}_{\mathcal{J}}}\right)$ for and any $\mathcal{J}$ such that $|\mathcal{J}|>0$. Then from (b) we see that for any $i>0, K \dashv_{\Gamma}{ }^{p} H^{\cdot}\left(\Upsilon_{!}^{\mathbf{s}} f_{!}^{i} f^{i *} \overline{\mathcal{L}}\right)$ if and only if $K \dashv_{\Gamma}{ }^{p} H^{\cdot}\left(\Upsilon_{!}^{\mathbf{s}} f_{!}^{i+1} f^{i+1 *} \overline{\mathcal{L}}\right)$. Since $K \bigwedge_{\Gamma}^{p} H^{\cdot}\left(\Upsilon_{!}^{\mathbf{s}} f_{!}^{i} f^{i *} \overline{\mathcal{L}}\right)$ with large $i$ it follows that $K \bigwedge_{\Gamma}{ }^{p} H^{\cdot}\left(\Upsilon_{!}^{\mathbf{s}} f_{!}^{1} f^{1 *} \overline{\mathcal{L}}\right)$. Using again (b) (with $i=0$ ) we see that $K \dashv_{\Gamma}{ }^{p} H^{\cdot}\left(\Upsilon_{!}^{\mathbf{s}} f_{!}^{0} f^{0 *} \overline{\mathcal{L}}\right)$ hence $K \dashv_{\Gamma}{ }^{p} H^{*}\left(\Upsilon_{!}^{\mathbf{s}} \overline{\mathcal{L}}\right)$. Thus, $K$ satisfies $3.2(\mathrm{v})$. The equivalence of $3.2(\mathrm{iii}), 3.2(\mathrm{v})$ is proved.

3.5. Let $\mathbf{s}=\left(s_{1}, s_{2}, \ldots, s_{r}\right)$ be a sequence in $\mathbf{I}$ such that $\mathcal{L} \in \mathcal{S}(T)^{[\mathbf{s}] F}$. Assume that $r \geq 2, h \in[2, r] \cap \mathcal{I}_{\mathbf{s}}, s_{h-1}=s_{h}$. Let $\mathbf{s}^{\prime}, \overline{\mathcal{L}}^{\prime}, \beta$ be as in 2.14 . We have $\Upsilon^{\mathbf{s}}=\Upsilon^{\mathbf{s}^{\prime}} \beta$ and using 2.14(a) we have $\Upsilon_{!}^{\mathbf{s}}(\overline{\mathcal{L}})=\Upsilon_{!}^{\mathbf{s}^{\prime}} \beta_{!} \beta^{*} \overline{\mathcal{L}}^{\prime}$. Since $\beta$ is a projective line bundle we have an exact sequence in $\mathcal{M}_{\Gamma}\left(\mathcal{P}_{J}\right)$ :

$$
\cdots \rightarrow{ }^{p} H^{j-2}\left(\Upsilon_{!}^{\mathbf{s}^{\prime}} \overline{\mathcal{L}}^{\prime}\right)(-1) \rightarrow{ }^{p} H^{j}\left(\Upsilon_{!}^{\mathbf{s}} \overline{\mathcal{L}}\right) \rightarrow{ }^{p} H^{j}\left(\Upsilon_{!}^{\mathbf{s}^{\prime}} \overline{\mathcal{L}}^{\prime}\right) \rightarrow \ldots
$$

3.6. Let $\mathbf{s}=\left(s_{1}, s_{2}, \ldots, s_{r}\right)$ be a sequence in $\mathbf{I}$ such that $\mathcal{L} \in \mathcal{S}(T)^{[\mathbf{s}] F}$. Assume that $r \geq 2, h \in[2, r], h \notin \mathcal{I}_{\mathbf{s}}, s_{h-1}=s_{h}$. Let $Z^{1}, Z^{2}, \beta, \beta^{\prime}, \mathbf{s}^{\prime}, \overline{\mathcal{L}}^{\prime}$ be as in 2.15 ; let $f_{1}: Z^{1} \rightarrow \mathcal{Z}^{\mathbf{s}}, f_{2}: Z^{2} \rightarrow \mathcal{Z}^{\mathbf{s}}$ be the inclusions. We have a distinguished triangle

$$
\left(\Upsilon_{!}^{\mathbf{s}} f_{1 !} f_{1}^{*} \overline{\mathcal{L}}, \Upsilon_{!}^{\mathbf{s}} \overline{\mathcal{L}}, \Upsilon_{!}^{\mathbf{s}} f_{2 !} f_{2}^{*} \overline{\mathcal{L}}\right)
$$

We have $\Upsilon_{!}^{\mathrm{s}} f_{1 !}=e_{!} \beta_{\text {! }}$ where $e: \mathcal{B}^{r} \rightarrow \mathcal{P}_{J}$ is $\left(B_{0}, B_{1}, \ldots, B_{h-2}, B_{h}, \ldots, B_{r}\right) \mapsto$ $P_{B_{0}, J}$. Using 2.15 (a) we have $\Upsilon_{!}^{\mathbf{s}} f_{1 !} f_{1}^{*} \overline{\mathcal{L}}=e_{!} \beta_{!}\left(\left.\overline{\mathcal{L}}\right|_{Z^{1}}\right)=0$. Hence the distinguished triangle above yields $\Upsilon_{!}^{\mathbf{s}} \overline{\mathcal{L}}=\Upsilon_{!}^{\mathbf{s}} f_{2 !} f_{2}^{*} \overline{\mathcal{L}}$. We have $\Upsilon^{\mathbf{s}} f_{2}=\Upsilon^{\mathbf{s}^{\prime}} \beta^{\prime}$. Using 2.15(b) we have

$$
\Upsilon_{!}^{\mathbf{s}} f_{2 !} f_{2}^{*} \overline{\mathcal{L}}=\Upsilon_{!}^{\mathbf{s}^{\prime}} \beta_{!}^{\prime}\left(\left.\overline{\mathcal{L}}\right|_{Z^{2}}\right)=\Upsilon_{!}^{\mathbf{s}^{\prime}} \beta_{!}^{\prime} \beta^{\prime *} \overline{\mathcal{L}}^{\prime}=\Upsilon_{!}^{\mathbf{s}^{\prime}}\left(\overline{\mathcal{L}}^{\prime} \otimes \beta_{!}^{\prime} \beta^{\prime *} \overline{\mathbf{Q}}_{l}\right)
$$

We see that

$$
\Upsilon_{!}^{\mathbf{s}} \overline{\mathcal{L}}=\Upsilon_{!}^{\mathbf{s}^{\prime}} \overline{\mathcal{L}}^{\prime}[-2](-1)
$$


Hence for any $j$ we have

$$
{ }^{p} H^{j}\left(\Upsilon_{!}^{\mathbf{s}} \overline{\mathcal{L}}\right)={ }^{p} H^{j-2}\left(\Upsilon_{!}^{\mathbf{s}^{\prime}} \overline{\mathcal{L}}^{\prime}\right)(-1)
$$

in $\mathcal{M}_{\Gamma}\left(\mathcal{P}_{J}\right)$.

3.7. Assume that 3.2(iv) holds. We show that 3.2(i) holds.

We may assume that $K \dashv_{\Gamma}{ }^{p} H^{\cdot}\left(\Pi_{!}^{\mathbf{s}} \mathcal{L}_{\mathbf{s}}\right)$ for some sequence $\mathbf{s}=\left(s_{1}, s_{2}, \ldots, s_{r}\right)$ in I such that $\mathcal{L} \in \mathcal{S}(T)^{[\mathrm{s}]}$ and that $r$ is minimum possible. From the proof in 3.4 we see that $K \dashv_{\Gamma}{ }^{p} H^{\cdot}\left(\Upsilon_{!}^{\mathbf{s}} \overline{\mathcal{L}}\right)$ and that $r$ is also minimal for this property.

Assume first that $l\left(s_{1} s_{2} \ldots s_{r}\right)<r$. We can find $h \in[2, r]$ such that

$$
l\left(s_{h} s_{h+1} \ldots s_{r}\right)=r-h+1, l\left(s_{h-1} s_{h} \ldots s_{r}\right)<r-h+2 .
$$

We can find $s_{h}^{\prime}, s_{h+1}^{\prime}, \ldots, s_{r}^{\prime}$ in $\mathbf{I}$ such that

$$
s_{h}^{\prime} s_{h+1}^{\prime} \ldots s_{r}^{\prime}=s_{h} s_{h+1} \ldots s_{r}=y
$$

and $s_{h}^{\prime}=s_{h-1}$. Let

$$
\mathbf{u}^{\prime}=\left(s_{1}, s_{2}, \ldots, s_{h-1}, s_{h}^{\prime}, s_{h+1}^{\prime}, \ldots, s_{r}^{\prime}\right), \mathbf{u}^{\prime \prime}=\left(s_{1}, s_{2}, \ldots, s_{h-1}, y\right) .
$$

From 3.3(a) we see that $\Pi_{!}^{\mathbf{s}} \mathcal{L}_{\mathbf{s}}=\Pi_{!}^{\mathbf{u}^{\prime}} \mathcal{L}_{\mathbf{u}^{\prime}}=\Pi_{!}^{\mathbf{u}^{\prime \prime}} \mathcal{L}_{\mathbf{u}^{\prime \prime}}$. Hence we may assume that $s_{h}=s_{h-1}$.

If $h \in \mathcal{I}_{\mathbf{s}}$, then using 3.5(a) we see that $K \dashv_{\Gamma}{ }^{p} H^{\cdot}\left(\Upsilon_{!}^{\mathbf{s}^{\prime}} \overline{\mathcal{L}}^{\prime}\right)$ (notation of 3.5); since $\mathbf{s}^{\prime}$ has $r-1$ terms this is a contradiction. If $h \notin \mathcal{I}_{\mathbf{s}}$, then using 3.6 we see that $K \dashv_{\Gamma}^{p} H^{\cdot}\left(\Upsilon_{!}^{\mathbf{s}^{\prime}} \overline{\mathcal{L}}^{\prime}\right)$ (notation of 3.6); since $\mathbf{s}^{\prime}$ has $r-2$ terms this is a contradiction.

We see that $l\left(s_{1} s_{2} \ldots s_{r}\right)=r$. Using $3.3\left(\right.$ a) repeatedly we see that ${ }^{p} H^{j}\left(\Pi_{!}^{\mathbf{s}} \mathcal{L}_{\mathbf{s}}\right)=$ ${ }^{p} H^{j}\left(\Pi_{!}^{\mathbf{w}} \mathcal{L}_{\mathrm{w}}\right)$ where $\mathbf{w}=\left(w_{1}\right), w_{1}=s_{1} s_{2} \ldots s_{r}$. Thus, $3.2(\mathrm{i})$ holds.

Since the implication $3.2(\mathrm{i}) \Longrightarrow 3.2(\mathrm{ii})$ is obvious and the equivalence of $3.2(\mathrm{v})$, 3.2 (vi) follows from 3.2(a) we see that the equivalence of 3.2(i) $-3.2(\mathrm{vi})$ is established.

For an object $A$ of $\mathcal{M}_{\Gamma}\left(\mathcal{P}_{J}\right)$ we write $A \in \mathbb{S}^{\prime}\left(\mathcal{P}_{J}\right)$ instead of " $A$ satisfies the equivalent conditions of 3.2(i)-3.2(vi) for some $\mathcal{L} \in \mathcal{S}(T)$ ".

3.8. The results in this and the next subsection are not used in the subsequent sections.

Let $\mathbf{s}=\left(s_{1}, s_{2}, \ldots, s_{r}\right)$ be a sequence in $\mathbf{I}$ such that $\mathcal{L} \in \mathcal{S}(T)^{[\mathbf{s}] F}, s_{1} \in J$. Let $\mathbf{s}^{\prime}=\left(s_{1}^{\prime}, s_{2}^{\prime}, \ldots, s_{r}^{\prime}\right)$ where $s_{i}^{\prime}=s_{i+1}$ for $i \in[1, r-1]$ and $s_{r}^{\prime}=\mathbf{c}\left(s_{1}\right)$ where c : $W \rightarrow W$ is as in 2.4. Let $\mathcal{L}^{\prime}=s_{1}^{*} \mathcal{L}$. We have $\mathcal{L}^{\prime} \in \mathcal{S}(T)^{\left[\mathbf{s}^{\prime}\right] F}$. Let $\overline{\mathcal{L}}$ be the local system on $\mathcal{Z}^{\mathrm{s}}$ defined in 2.11 and let $\overline{\mathcal{L}}^{\prime}$ be the analogous local system on $\mathcal{Z}^{\mathrm{s}}$ defined in terms of $\mathcal{L}^{\prime}$. We show:

$$
\Upsilon_{!}^{\mathbf{s}} \overline{\mathcal{L}} \cong \Upsilon_{!}^{\mathbf{s}^{\prime}} \overline{\mathcal{L}}^{\prime}
$$

Define $\mathcal{I}_{\mathbf{s}^{\prime}}^{\prime}$ in terms of $\mathbf{s}^{\prime}, \mathcal{L}^{\prime}$ in the same way as $\mathcal{I}_{\mathbf{s}}$ was defined in 2.4 in terms of $\mathbf{s}, \mathcal{L}$. If $i \in[2, r]$ we have $i \in \mathcal{I}_{\mathbf{s}}$ if and only if $i-1 \in \mathcal{I}_{\mathbf{s}^{\prime}}^{\prime}$. Moreover, we have $1 \in \mathcal{I}_{\mathbf{s}}$ if and only if $r \in \mathcal{I}_{\mathbf{s}^{\prime}}$. It follows that $f: \mathcal{Z}^{\mathbf{s}} \rightarrow \mathcal{Z}^{\mathbf{s}^{\prime}},\left(B_{0}, B_{1}, \ldots, B_{r}\right) \mapsto$ $\left(B_{1}, B_{2}, \ldots, B_{r}, F\left(B_{1}\right)\right)$, is well defined. From the definitions we see that $f^{*} \overline{\mathcal{L}}^{\prime} \cong \overline{\mathcal{L}}$. Hence $\Upsilon_{!}^{\mathbf{s}} \overline{\mathcal{L}}=\Upsilon_{!}^{\mathbf{s}} f^{*} \overline{\mathcal{L}}^{\prime}$. It remains to show that $\Upsilon^{\mathbf{s}} f=\Upsilon^{\mathbf{s}^{\prime}}$. The first (resp. second) map takes $\left(B_{0}, B_{1}, \ldots, B_{r}\right)$ to $P_{B_{1}, J}$ (resp. $\left.P_{B_{0}, J}\right)$. It is enough to show that $P_{B_{1}, J}=P_{B_{0}, J}$. This follows from the fact that $\operatorname{pos}\left(B_{0}, B_{1}\right) \in J$. 
3.9. Let $\mathbf{s}=\left(s_{1}, s_{2}, \ldots, s_{r}\right)$ be a sequence in $\mathbf{I}$ such that $\mathcal{L} \in \mathcal{S}(T)^{[\mathbf{s}] F}$. Let $s \in$ I be such that $s \notin W_{\mathcal{L}}$. Let $\mathbf{u}=\left(s, s_{1}, s_{2}, \ldots, s_{r}, \mathbf{c}(s)\right), \mathcal{L}^{\prime}=s^{*} \mathcal{L}$. Let $\mathbf{v}=$ $\left(s, s, s_{1}, s_{2}, \ldots, s_{r}\right)$. We have $\mathcal{L}^{\prime} \in \mathcal{S}(T)^{[\mathbf{u}] F}, \mathcal{L} \in \mathcal{S}(T)^{[\mathbf{v}] F}$. Let $\overline{\mathcal{L}}$ be as in 2.11; let $\overline{\mathcal{L}}^{\prime}, \overline{\mathcal{L}}^{\prime \prime}$ be the analogous local systems on $\mathcal{Z}^{\mathbf{u}}, \mathcal{Z}^{\mathbf{v}}$ defined in terms of $\mathcal{L}^{\prime}, \mathcal{L}$. We show that

$$
\Upsilon_{!}^{\mathbf{s}} \overline{\mathcal{L}}[-2](-1)=\Upsilon_{!}^{\mathbf{u}} \overline{\mathcal{L}}^{\prime}
$$

From 3.8 we have $\Upsilon_{!}^{\mathbf{u}} \overline{\mathcal{L}}^{\prime}=\Upsilon_{!}^{\mathbf{v}} \overline{\mathcal{L}}^{\prime \prime}$. From 3.6(a) we have $\Upsilon_{!}^{\mathbf{v}} \overline{\mathcal{L}}^{\prime \prime}=\Upsilon_{!}^{\mathbf{s}} \overline{\mathcal{L}}[-2](-1)$ and (a) follows. We see that

$$
{ }^{p} H^{j}\left(\Upsilon_{!}^{\mathbf{u}} \overline{\mathcal{L}}^{\prime}\right)={ }^{p} H^{j-2}\left(\Upsilon_{!}^{\mathbf{s}} \overline{\mathcal{L}}\right)(-1) .
$$

\section{The Class $\mathbb{S}\left(\mathcal{P}_{J}\right)$ of Simple objects in $\mathcal{M}_{\Gamma}\left(\mathcal{P}_{J}\right)$}

4.1. In this section we fix $J \subset \mathbf{I}$.

In 1977 the author generalized the partition $\left(\mathcal{B}_{w}\right)_{w \in W}$ of $\mathcal{B}$ (see 2.5) by defining a partition of $\mathcal{P}_{J}$ into finitely many pieces stable under conjugation by $\Gamma$, as follows. To any $P \in \mathcal{P}_{J}$ we associate a sequence $P^{0} \supset P^{1} \supset P^{2} \supset \ldots$ in $\mathcal{P}$ by

$$
P^{0}=P, \quad P^{n}=\left(P^{n-1}\right)^{F\left(P^{n-1}\right)} \text { for } n \geq 1,
$$

a sequence $J_{0} \supset J_{1} \supset J_{2} \supset \ldots$ of subsets of $\mathbf{I}$ by $P^{n} \in \mathcal{P}_{J_{n}}$ and a sequence $w_{0}, w_{1}, w_{2}, \ldots$ in $W$ by

We have

$$
w_{n}=\operatorname{pos}\left(P^{n}, F\left(P^{n}\right)\right) .
$$

(a) $J_{0}=J$

(b) $J_{n}=J_{n-1} \cap w_{n-1} \mathbf{c}\left(J_{n-1}\right) w_{n-1}^{-1}$ for $n \geq 1$. (see $\left.1.3(\mathrm{a})\right)$,

(c) $w_{n} \in{ }^{J_{n}} W^{\mathbf{c}\left(J_{n}\right)}$ for $n \geq 0$.

Clearly, for $n \geq|\mathbf{I}|$ we have $P^{n}=P^{n+1}=\ldots$ hence

(d) $w_{n}=w_{n+1}=\ldots$ and $J_{n}=J_{n+1}=\ldots$.

We set $P^{\infty}=P^{n}$ for $n \geq|\mathbf{I}|, w_{\infty}=w_{n}$ for $n \geq|\mathbf{I}|, J_{\infty}=J_{n}$ for $n \geq|\mathbf{I}|$.

For any $\mathbf{t}=\left(J_{n}, w_{n}\right)_{n \geq 0}$ where $J_{0} \supset J_{1} \supset J_{2} \supset \ldots$ are subsets of $J$ satisfying (a) and $w_{0}, w_{1}, w_{2}, \ldots$ are elements of $W$ satisfying (b),(c) let $\mathcal{P}_{J}^{\mathrm{t}}$ be the set of all $P \in \mathcal{P}_{J}$ which give rise to $\mathbf{t}$ by the procedure above. Let $\mathcal{T}^{\prime}(J, \mathbf{c})$ be the set of all sequences $\mathbf{t}$ as above such that $\mathcal{P}_{J}^{\mathbf{t}} \neq \emptyset$. From (d) we see that $\mathcal{T}^{\prime}(J, \mathbf{c})$ is a finite set. From (a),(b) we see that for $\left(J_{n}, w_{n}\right)_{n \geq 0} \in \mathcal{T}^{\prime}(J, \mathbf{c})$, the $J_{n}$ are uniquely determined by the $w_{n}$. The locally closed subvarieties $\mathcal{P}_{J}^{\mathrm{t}}, \mathbf{t} \in \mathcal{T}^{\prime}(J, \mathbf{c})$, form the desired partition of $\mathcal{P}_{J}$. (See [L5, I, 1.3, 1.4] for some examples in the classical groups.)

4.2. In this subsection we review some results in R. Bédard's Ph.D. Thesis (M.I.T. 1983); see also BE].

(a) $\mathcal{T}^{\prime}(J, \mathbf{c})$ is precisely the set of all $\left(J_{n}, w_{n}\right)_{n \geq 0}$ with $J_{n} \subset \mathbf{I}, w_{n} \in W$ such that 4.1(a),(b), (c) hold and $w_{n} \in W_{J_{n}} w_{n-1} W_{\mathbf{c}\left(J_{n-1}\right)}$ for $n \geq 1$.

(With notation in [L5, I, 2.2] we have $\mathcal{T}^{\prime}(J, \mathbf{c})=\mathcal{T}\left(\mathbf{c}(J), \mathbf{c}^{-1}\right)$.)

(b) The assignment $\left(J_{n}, w_{n}\right)_{n \geq 0} \mapsto w_{\infty}$ defines a bijection $\mathcal{T}^{\prime}(J, \mathbf{c}) \stackrel{\sim}{\rightarrow}{ }^{J} W$.

(c) Let $z \in{ }^{J} W^{\mathbf{c}(J)}, J_{1}=J \cap z \mathbf{c}(J) z^{-1}$. Let $V=\left\{P \in \mathcal{P}_{J} ; \operatorname{pos}(P, F(P))=z\right.$, $V^{\prime}=\left\{Q \in \mathcal{P}_{J_{1}} ; \operatorname{pos}(Q, F(Q)) \in z W_{\mathbf{c}(J)}\right\}$. Then $f: V \rightarrow V^{\prime}, P \mapsto P^{1}:=P^{F(P)}$ is an isomorphism.

Define $V^{\prime} \rightarrow \mathcal{P}_{J}$ by $Q \mapsto P$ where $P$ is the unique parabolic in $\mathcal{P}_{J}$ such that $Q \subset P$. We have automatically $P \in V$ hence $Q \mapsto P$ is a map $f^{\prime}: V^{\prime} \rightarrow V$. Clearly 
$f^{\prime} f=1$. We show $f f^{\prime}=1$. It is enough to show that, if $Q, P$ are as above, then $P^{F(P)}=Q$. We have $\operatorname{pos}(Q, F(Q))=z u$ where $u \in W_{\mathbf{c}(J)}$. We can find $B_{0}, B_{1} \in \mathcal{B}$ such that $B_{0} \subset Q, B_{1} \subset F(Q), \operatorname{pos}\left(B_{0}, B_{1}\right)=z u$. Since $l(z u)=l(z)+l(u)$ we can find $B_{2} \in \mathcal{B}$ such that $\operatorname{pos}\left(B_{0}, B_{2}\right)=z, \operatorname{pos}\left(B_{2}, B_{1}\right)=u$. Since $u \in W_{\mathbf{c}(J)}$ and $B_{1} \subset F(P)$ we have $B_{2} \subset F(P)$. Since $B_{0} \subset P, B_{2} \subset F(P), \operatorname{pos}\left(B_{0}, B_{2}\right)=z$, we have $B_{0} \subset P^{F(P)}$. Since $Q, P^{F(P)}$ are in $\mathcal{P}_{J_{1}}$ and both contain $B_{0}$ we have $Q=P^{F(P)}$. This proves (c).

Let $\mathbf{t}=\left(J_{n}, w_{n}\right)_{n \geq 0} \in \mathcal{T}^{\prime}(J, \mathbf{c})$. For $m \geq 0$ we set $\mathbf{t}_{m}=\left(J_{n}^{\prime}, w_{n}^{\prime}\right)_{n \geq 0}$ where $J_{n}^{\prime}=J_{n+m}, w_{n}^{\prime}=w_{n+m}$. We have $\mathbf{t}_{m} \in \mathcal{T}^{\prime}\left(J_{m}, \mathbf{c}\right)$. We set $\mathbf{t}_{\infty}=\left(J_{n}^{\prime}, w_{n}^{\prime}\right)_{n \geq 0}$ where $J_{n}^{\prime}=J_{\infty}, w_{n}^{\prime}=w_{\infty}$. We have $\mathbf{t}_{\infty} \in \mathcal{T}^{\prime}\left(J_{\infty}, \mathbf{c}\right)$. Clearly, $P \mapsto P^{1}$ is a map

$$
\vartheta: \mathcal{P}_{J}^{\mathrm{t}} \rightarrow \mathcal{P}_{J_{1}}^{\mathrm{t}_{1}} \text {. }
$$

(d) The map $P \mapsto P^{1}$ is an isomorphism $\mathcal{P}_{J}^{\mathbf{t}} \stackrel{\sim}{\rightarrow} \mathcal{P}_{J_{1}}^{\mathrm{t}_{1}}$. The map $P \mapsto P^{\infty}$ is an isomorphism $\mathcal{P}_{J}^{\mathrm{t}} \stackrel{\sim}{\rightarrow} \mathcal{P}_{J_{\infty}}^{\mathrm{t}_{\infty}}$.

The first assertion of (d) follows from (c). The second assertion follows using the first assertion repeatedly.

(e) Let $\mathbf{t}=\left(J_{n}, w_{n}\right)_{n \geq 0} \in \mathcal{T}^{\prime}(J, \mathbf{c})$ be such that $J_{n}=J$ and $w_{n}=w$ for all $n \geq 0$ where $w \in W$. We have $\mathbf{c}(J)=w^{-1} J w, w \in{ }^{J} W^{\mathbf{c}(J)}$. If $P \in \mathcal{P}_{J}^{\mathrm{t}}$, then $P^{n}=P$ for $n \geq 0$ and $\operatorname{pos}(P, F(P))=w$. From $P=P^{F(P)}$ we see that $P, F(P)$ have a common Levi. We have $\mathcal{P}_{J}^{\mathbf{t}}=\left\{P \in \mathcal{P}_{J} ; \operatorname{pos}(P, F(P))=w\right\}$.

(f) Let $\left(J_{n}, w_{n}\right)_{n \geq 0} \in \mathcal{T}^{\prime}(J, \mathbf{c})$. For $n \geq 0$ we have $w_{n}=\min \left(W_{J} w_{\infty} W_{\mathbf{c}\left(J_{n}\right)}\right)$.

4.3. In the setup of $4.2(\mathrm{e})$ we show:

$$
\dot{w}^{-1} L_{J} \dot{w}=L_{\mathbf{c}(J)}=F\left(L_{J}\right) .
$$

From $\operatorname{pos}\left(P_{J}, \dot{w} P_{\mathbf{c}(J)} \dot{w}^{-1}\right)=w$ we see that $P_{J}, \dot{w} P_{\mathbf{c}(J)} \dot{w}^{-1}$ have a common Levi subgroup containing $T$ which must be $L_{J}$ and also $\dot{w} L_{\mathbf{c}(J)} \dot{w}^{-1}$.

Let

$$
\tilde{\mathcal{P}}_{J}^{\mathrm{t}}=\left\{g U_{P_{J}} \in G / U_{P_{J}} ; g^{-1} F(g) \in U_{P_{J}} \dot{w} U_{P_{\mathbf{c}(J)}}\right\} .
$$

Define $F^{\prime}: L_{J} \rightarrow L_{J}$ by $g \mapsto \dot{w} F(g) \dot{w}^{-1}$. This is the Frobenius map for an $\mathbf{F}_{q^{-}}$ rational structure on $L_{J}$. We set

$$
L_{J}^{F^{\prime}}=\left\{l \in L_{J} ; F^{\prime}(l)=l\right\} .
$$

The finite group $L_{J}^{F^{\prime}}$ acts freely on $\tilde{\mathcal{P}}_{J}^{\mathrm{t}}$ by $l: g U_{P_{J}} \mapsto g l^{-1} U_{P_{J}}$ and the map $f: \tilde{\mathcal{P}}_{J}^{\mathrm{t}} \rightarrow \mathcal{P}_{J}^{\mathrm{t}}, g U_{P_{J}} \mapsto g P_{J} g^{-1}$ is constant on the orbits of this action. We show:

(b) $f$ is a principal $L_{J}^{F^{\prime}}$-bundle.

We only show this at the level of sets. If $P \in \mathcal{P}_{J} ; \operatorname{pos}(P, F(P))=w$, we have $P=g P_{J} g^{-1}$ where $g \in G$ satisfies

$$
\begin{aligned}
w & =\operatorname{pos}\left(g P_{J} g^{-1}, F(g) F\left(P_{J}\right) F\left(g^{-1}\right)\right) \\
& =\operatorname{pos}\left(P_{J}, g^{-1} F(g) P_{\mathbf{c}(J)} F\left(g^{-1}\right) g\right)=\operatorname{pos}\left(P_{J}, \dot{w} P_{\mathbf{c}(J)} \dot{w}^{-1}\right) .
\end{aligned}
$$

Hence there exists $y \in P_{J}$ such that $g^{-1} F(g) P_{\mathbf{c}(J)} F\left(g^{-1}\right) g=y \dot{w} P_{\mathbf{c}(J)} \dot{w}^{-1} y^{-1}$ hence $g^{-1} F(g) \in P_{J} \dot{w} P_{\mathbf{c}(J)}$; that is, $g^{-1} F(g) \in l^{\prime} U_{P_{J}} \dot{w} U_{P_{\mathbf{c}(J)}}$ for some $l^{\prime} \in L_{J}$. (We use (a).) By Lang's theorem for $F^{\prime}$ we can find $l \in L_{J}$ such that $l^{-1} F^{\prime}(l)=l^{\prime}$. Then $g l^{-1} U_{P_{J}} \in \tilde{\mathcal{P}}_{J}^{\mathrm{t}}$. We see that $f$ is surjective. 
Assume that $g U_{P_{J}}, g^{\prime} U_{P_{J}}$ in $\tilde{\mathcal{P}}_{J}^{\mathrm{t}}$ have the same image under $f$ that is $g P_{J} g^{-1}=$ $g^{\prime} P_{J} g^{\prime-1}$. Then $g^{\prime}=g p^{-1}$ where $p \in P_{J}$. We may assume that $g^{\prime}=g l^{-1}, l \in L_{J}$. We have $g^{-1} F(g) \in P_{J} \dot{w} P_{\mathbf{c}(J)}$ and $\left(g l^{-1}\right)^{-1} F\left(g l^{-1}\right) \in U_{P_{J}} \dot{w} U_{P_{\mathbf{c}(J)}}$ that is

$$
g^{-1} F(g)=U_{P_{J}} l^{-1} \dot{w} F(l) U_{P_{\mathbf{c}(J)}} \text { and } U_{P_{J}} l^{-1} F^{\prime}(l) \dot{w} U_{P_{\mathbf{c}(J)}}=U_{P_{J}} \dot{w} U_{P_{\mathbf{c}(J)}} .
$$

Using [L3, 3.2] we deduce $l^{-1} F^{\prime}(l) \dot{w}=\dot{w}$ hence $l \in L_{J}^{F^{\prime}}$.

Let

${ }^{\prime} \tilde{\mathcal{P}}_{J}^{\mathrm{t}}=\left\{g\left(U_{P_{J}} \cap F^{-1}\left(\dot{w}^{-1} U_{P_{J}} \dot{w}\right)\right) \in G /\left(U_{P_{J}} \cap F^{-1}\left(\dot{w}^{-1} U_{P_{J}} \dot{w}\right)\right) ; g^{-1} F(g) \in U_{P_{J}} \dot{w}\right\}$.

We show:

(c) The map $g\left(U_{P_{J}} \cap F^{-1}\left(\dot{w}^{-1} U_{P_{J}} \dot{w}\right)\right) \mapsto g U_{P_{J}}$ is an isomorphism $\gamma:{ }^{\prime} \tilde{\mathcal{P}}_{J}^{\mathrm{t}} \stackrel{\sim}{\rightarrow} \tilde{\mathcal{P}}_{J}^{\mathrm{t}}$.

We only show this at the level of sets. Let $g U_{P_{J}} \in \tilde{\mathcal{P}}_{J}^{\mathrm{t}}$. We have $g^{-1} F(g)=$ $u \dot{w} F\left(u^{\prime}\right)$ for some $u \in U_{P_{J}}, u^{\prime} \in U_{P_{J}}$. Then $\left(g u^{\prime-1}\right)^{-1} F\left(g u^{\prime-1}\right)=u^{\prime} u \dot{w}$ so that $\gamma\left(g u^{\prime-1}\left(U_{P_{J}} \cap F^{-1}\left(\dot{w}^{-1} U_{P_{J}} \dot{w}\right)\right)\right)=g U_{P_{J}}$. We see that $\gamma$ is surjective. The injectivity is immediate.

Next we show:

(d) $\mathcal{P}_{J}^{\mathrm{t}}$ is a smooth variety of pure dimension equal to $\operatorname{dim} U_{P_{J}}-\operatorname{dim}\left(U_{P_{J}} \cap\right.$ $\left.F^{-1}\left(\dot{w}^{-1} U_{P_{J}} \dot{w}\right)\right)$ and its connected components are permuted transitively by the $\Gamma$ action on $\mathcal{P}_{J}^{\mathrm{t}}$.

By (b), (c) it is enough to show that ' $\tilde{\mathcal{P}}_{J}^{\mathrm{t}}$ is smooth, of pure dimension equal to $\operatorname{dim} U_{P_{J}}-\operatorname{dim}\left(U_{P_{J}} \cap F^{-1}\left(\dot{w}^{-1} U_{P_{J}} \dot{w}\right)\right)$ and its connected components are permuted transitively by the $\Gamma$-action

$$
g_{0}: g\left(U_{P_{J}} \cap F^{-1}\left(\dot{w}^{-1} U_{P_{J}} \dot{w}\right)\right) \mapsto g_{0} g\left(U_{P_{J}} \cap F^{-1}\left(\dot{w}^{-1} U_{P_{J}} \dot{w}\right)\right)
$$

on ' $\tilde{\mathcal{P}}_{J}^{\mathrm{t}}$. This follows from the fact that $\left\{g \in G ; g^{-1} F(g) \in U_{P_{J}} \dot{w}\right\}$ is smooth of dimension $\operatorname{dim} U_{P_{J}}$ and $U_{P_{J}} \dot{w}$ is connected.

4.4. We now consider a general $\mathbf{t}=\left(J_{n}, w_{n}\right)_{n \geq 0} \in \mathcal{T}^{\prime}(J, \mathbf{c})$. Let $\mathcal{P}_{J}^{\mathbf{t}} \stackrel{\sim}{\rightarrow} \mathcal{P}_{J_{\infty}}^{\mathbf{t}}$ be the isomorphism in $4.2(\mathrm{~d})$. By 4.3 for $\mathbf{t}_{\infty}$ instead of $\mathbf{t}, \tilde{\mathcal{P}}_{J_{\infty}}^{\mathbf{t}_{\infty}}$ is defined. Let

$$
\tilde{\mathcal{P}}_{J}^{\mathrm{t}}:=\tilde{\mathcal{P}}_{J_{\infty}}^{\mathrm{t}_{\infty}}=\left\{g U_{P_{J_{\infty}}} \in G / U_{P_{J_{\infty}}} ; g^{-1} F(g) \in U_{P_{J_{\infty}}} \dot{w} U_{P_{\mathbf{c}\left(J_{\infty}\right)}}\right\} .
$$

Define $F^{\prime}: L_{J_{\infty}} \rightarrow L_{J_{\infty}}$ by $l \mapsto \dot{w}_{\infty} F(l) \dot{w}_{\infty}^{-1}$. (We have $\dot{w}_{\infty}^{-1} L_{J_{\infty}} \dot{w}_{\infty}=L_{\mathbf{c}\left(J_{\infty}\right)}=$ $F\left(L_{J_{\infty}}\right)$; see $4.3(\mathrm{a})$.) Let $\Lambda=L_{J_{\infty}}^{F^{\prime}}$. The finite group $\Gamma \times \Lambda$ acts on $\tilde{\mathcal{P}}_{J}^{\mathrm{t}}$ by

$$
\left(g_{0}, l\right): g U_{P_{J_{\infty}}} \mapsto g_{0} g l^{-1} U_{P_{J_{\infty}}}
$$

and on $\mathcal{P}_{J}^{\mathrm{t}}$ by $\left(g_{0}, l\right): P \mapsto g_{0} P g_{0}^{-1}$. From $4.3(\mathrm{~b})$ we see that:

(a) The map $f: \tilde{\mathcal{P}}_{J}^{\mathrm{t}} \rightarrow \mathcal{P}_{J}^{\mathrm{t}}, g U_{P_{J_{\infty}}} \mapsto g P_{J} g^{-1}$ (which is compatible with the $\Gamma \times \Lambda$-actions) is a principal $\Lambda$-bundle.

From 4.3(d) we see that:

(b) $\mathcal{P}_{J}^{\mathrm{t}}$ is a smooth variety of pure dimension equal to $\operatorname{dim} U_{P_{J_{\infty}}}-\operatorname{dim}\left(U_{P_{J_{\infty}}} \cap\right.$ $\left.F^{-1}\left(\dot{w}_{\infty}^{-1} U_{P_{J_{\infty}}} \dot{w}_{\infty}\right)\right)$ and its connected components are permuted transitively by the $\Gamma$-action on $\mathcal{P}_{J}^{\mathrm{t}}$.

Let $M$ be a finite dimensional $\Lambda$-irreducible module over $\overline{\mathbf{Q}}_{l}$. We view $M$ as a $\Gamma \times \Lambda$-module with $\Gamma$ acting trivially and we form the $\Gamma \times \Lambda$-equivariant local system $M_{\tilde{\mathcal{P}}_{J}^{\mathrm{t}}}$ on $\tilde{\mathcal{P}}_{J}^{\mathrm{t}}$ as in 1.6. Using (a) we see that there is a well defined $\Gamma \times \Lambda$ equivariant local system $\underline{M}$ on $\mathcal{P}_{J}^{\mathrm{t}}$ with trivial action of $\Lambda$ such that $f^{*} \underline{M}=M_{\tilde{\mathcal{P}}_{J}^{\mathrm{t}}}$ as $\Gamma \times \Lambda$-equivariant local systems. We will regard $\underline{M}$ as a $\Gamma$-equivariant local system. 
Let $d=\operatorname{dim} \mathcal{P}_{J}^{\mathrm{t}}($ see $(\mathrm{b}))$. We show:

(c) $\underline{M}[d]$ (an object of $\mathcal{M}_{\Gamma}\left(\mathcal{P}_{J}^{\mathbf{t}}\right)$ by (b)) is simple.

Let $r: \tilde{C} \rightarrow C$ be a finite principal covering with finite group $H$. Assume that $C$ is connected. There is an obvious functor $E \mapsto E^{\prime}$ from $H$-modules of finite dimension over $\overline{\mathbf{Q}}_{l}$ to local systems on $C$ which are direct summands of $r_{!} \overline{\mathbf{Q}}_{l}$. If $E$ is irreducible, then $E^{\prime}$ is irreducible as a local system.

We apply this statement in the case where $C$ is a connected component of $\mathcal{P}_{J}^{\mathrm{t}}$, $\tilde{C}=f^{-1}(C)(f$ as in (a)), $r$ is the restriction of $f, H=\Lambda$ and $E=M$. Note that $E^{\prime}=\left.\underline{M}\right|_{C}$. We see that the local system $\left.\underline{M}\right|_{C}$ is irreducible. It remains to use the transitivity statement in (b).

For an object $A$ of $\mathcal{M}_{\Gamma}\left(\mathcal{P}_{J}^{\mathbf{t}}\right)$ we write $A \in \mathbb{S}\left(\mathcal{P}_{J}^{\mathbf{t}}\right)$ instead of " $A$ is isomorphic to $\underline{M}[d]$ for some $M$ as above."

From (c) we see that:

(d) $\underline{M}^{\sharp}[d]$ is a simple object of $\mathcal{M}_{\Gamma}\left(\mathcal{P}_{J}\right)$.

For an object $A$ of $\mathcal{M}_{\Gamma}\left(\mathcal{P}_{J}\right)$ we write $A \in \mathbb{S}\left(\mathcal{P}_{J}\right)$ instead of " $A$ is isomorphic to $\underline{M}^{\sharp}[d] \in \mathcal{M}_{\Gamma}\left(\mathcal{P}_{J}\right)$ for some $\mathbf{t} \in \mathcal{T}^{\prime}(J, \mathbf{c})$ and some $M$ as above."

4.5. For $w \in W$ we identify $\mathcal{B}_{w}$ with $Z^{(w)}$ as in 2.5 . We show:

(a) Let $a_{1}, a_{2}, b \in W$. Let $\mathcal{L} \in \mathcal{S}(T)^{a_{1} a_{2} F}$. Let $V$ be a locally closed $\Gamma$-stable subvariety of $\mathcal{B}_{b}$. Let $X_{a_{1}, a_{2}}=\left\{\left(B_{0}, B_{1}, B_{2}\right) \in Z^{\left(a_{1}, a_{2}\right)} ; B_{1} \in V\right\}$, (see 2.5). Define $\kappa: X_{a_{1}, a_{2}} \rightarrow V$ by $\left(B_{0}, B_{1}, B_{2}\right) \mapsto B_{1}$. Let $\mathcal{E}=\mathcal{L}_{\left(a_{1}, a_{2}\right)} \mid X_{a_{1}, a_{2}}$. Let $V^{\prime}$ be an algebraic variety with a $\Gamma$-action and let $m: V \rightarrow V^{\prime}$ be a morphism compatible with the $\Gamma$-actions. Let $A^{\prime}$ be a simple object of $\mathcal{M}_{\Gamma}\left(V^{\prime}\right)$ such that $A^{\prime} \dashv_{\Gamma}{ }^{p} H^{\cdot}((m \kappa) ! \mathcal{E})$. Then there exists $e \in W$ such that $(b F)^{*}\left(e^{*} \mathcal{L}\right) \cong e^{*} \mathcal{L}$ and $A^{\prime} \dashv_{\Gamma}{ }^{p} H^{\cdot}\left(\left.m_{!}\left(e^{*} \mathcal{L}\right)_{b}\right|_{V}\right)$.

We argue by induction on $l\left(a_{1}\right)$. If $l\left(a_{1}\right)=0$, then $a_{2}=b, \kappa$ is an isomorphism and the result is obvious (with $e=1$ ). Assume now that $l\left(a_{1}\right)>0$. We can find $s \in \mathbf{I}$ such that $l\left(a_{1}\right)>l\left(s a_{1}\right)$. Let $\mathcal{E}_{1}=\left.\left(s^{*} \mathcal{L}\right)_{\left(s a_{1}, a_{2} \mathbf{c}(s)\right.}\right|_{X_{s a_{1}, a_{2} \mathbf{c}(s)}}$.

Assume first that $l\left(a_{2} \mathbf{c}(s)\right)=l\left(a_{2}\right)+1$. We have an isomorphism $\iota: X_{a_{1}, a_{2}} \rightarrow$ $X_{s a_{1}, a_{2} \mathbf{c}(s)},\left(B_{0}, B_{1}, B_{2}\right) \mapsto\left(B_{0}^{\prime}, B_{1}, F\left(B_{0}^{\prime}\right)\right)$ where $B_{0}^{\prime} \in \mathcal{B}$ is defined by

$$
\operatorname{pos}\left(B_{0}, B_{0}^{\prime}\right)=s, \operatorname{pos}\left(B_{0}^{\prime}, B_{1}\right)=s a_{1} .
$$

Define $\kappa^{\prime}: X_{s a_{1}, a_{2} \mathbf{c}(s)} \rightarrow V$ by $\left(B_{0}, B_{1}, B_{2}\right) \mapsto B_{1}$. We have $\kappa=\kappa^{\prime} \iota, \iota^{*} \mathcal{E}_{1}=\mathcal{E}$ hence $\iota ! \mathcal{E}=\mathcal{E}_{1}$. Thus $(m \kappa) ! \mathcal{E}=\left(m \kappa^{\prime}\right) ! \mathcal{E}_{1}$ and $A^{\prime} \dashv_{\Gamma}{ }^{p} H^{\cdot}\left(\left(m \kappa^{\prime}\right) ! \mathcal{E}_{1}\right)$. By the induction hypothesis there exists $e^{\prime} \in W$ such that $(b F)^{*}\left(e^{\prime *} s^{*} \mathcal{L}\right) \cong e^{\prime *} s^{*} \mathcal{L}$ and $A^{\prime} \dashv_{\Gamma}{ }^{p} H^{*}\left(\left.m_{!}\left(e^{*} s^{*} \mathcal{L}\right)_{b}\right|_{V}\right)$. The result follows with $e=s e^{\prime}$.

Assume next that $l\left(a_{2} \mathbf{c}(s)\right)=l\left(a_{2}\right)-1$. We have a partition $X_{a_{1}, a_{2}}=X^{\prime} \cup$ $X^{\prime \prime}$ where $X^{\prime}$ (resp. $X^{\prime \prime}$ ) is the open (resp. closed) subset of $X_{a_{1}, a_{2}}$ defined by $\operatorname{pos}\left(B_{1}, F\left(B_{0}^{\prime}\right)\right)=a_{2}\left(\right.$ resp. $\left.\operatorname{pos}\left(B_{1}, F\left(B_{0}^{\prime}\right)\right)=a_{2} \mathbf{c}(s)\right)$. Let $j^{\prime}=\left.\kappa\right|_{X^{\prime}}, j^{\prime \prime}=\left.\kappa\right|_{X^{\prime \prime}}$. By general principles we have either

(c) $A^{\prime} \dashv_{\Gamma}^{p} H^{\cdot}\left(\left(m j^{\prime}\right) !\left(\left.\mathcal{E}\right|_{X^{\prime}}\right)\right)$ or

(d) $A^{\prime} \dashv_{\Gamma}{ }^{p} H^{\cdot}\left(\left(m j^{\prime \prime}\right) !\left(\left.\mathcal{E}\right|_{X^{\prime \prime}}\right)\right)$.

Assume that (d) holds. We have $j^{\prime \prime}=\kappa^{\prime \prime} \iota^{\prime \prime}$ where $\kappa^{\prime \prime}: X_{s a_{1}, a_{2} \mathbf{c}(s)} \rightarrow V$ is given by $\left(B_{0}, B_{1}, B_{2}\right) \mapsto B_{1}$ and $\iota^{\prime \prime}: X^{\prime \prime} \rightarrow X_{s a_{1}, a_{2} \mathbf{c}(s)}$ is $\left(B_{0}, B_{1}, B_{2}\right) \mapsto\left(B_{0}^{\prime}, B_{1}, F\left(B_{0}^{\prime}\right)\right)$ with $B_{0}^{\prime}$ as in (b). We have $\left.\mathcal{E}\right|_{X^{\prime \prime}}=\iota^{\prime \prime *} \mathcal{E}_{1}$. Now $\iota^{\prime \prime}$ is an affine line bundle hence $\iota_{!}^{\prime \prime}\left(\left.\mathcal{E}\right|_{X^{\prime \prime}}\right)=\mathcal{E}_{1}[-2](-1)$. Hence $A^{\prime} \dashv_{\Gamma}{ }^{p} H^{\cdot}\left(\left(\mapsto \kappa^{\prime \prime}\right) ! \mathcal{E}_{1}\right)$. By the induction hypothesis there exists $e^{\prime} \in W$ such that $(b F)^{*}\left(e^{* *} s^{*} \mathcal{L}\right) \cong e^{\prime *} s^{*} \mathcal{L}$ and $A^{\prime} \dashv_{\Gamma}$ ${ }^{p} H^{*}\left(\left.m_{!}\left(e^{*} s^{*} \mathcal{L}\right)_{b}\right|_{V}\right)$. The result follows with $e=s e^{\prime}$.

Assume now that (c) holds. We have $j^{\prime}=\kappa^{\prime} \iota^{\prime}$ where $\kappa^{\prime}: X_{s a_{1}, a_{2}} \rightarrow V$ is $\left(B_{0}, B_{1}, B_{2}\right) \mapsto B_{1}$ and $\iota^{\prime}: X^{\prime} \rightarrow X_{s a_{1}, a_{2}}$ is $\left(B_{0}, B_{1}, B_{2}\right) \mapsto\left(B_{0}^{\prime}, B_{1}, F\left(B_{0}^{\prime}\right)\right)$ 
with $B_{0}^{\prime}$ as in (b). Note that $\iota^{\prime}$ makes $X^{\prime}$ into the complement of a section of an affine line bundle over $X_{s a_{1}, a_{2}}$. If $s \notin W_{\mathcal{L}}$, then by an argument as in the proof of 2.15 we see that $\iota_{!}^{\prime}\left(\left.\mathcal{E}\right|_{X^{\prime}}\right)=0$ contradicting (c). Thus we may assume that $s \in W_{\mathcal{L}}$. Then $\mathcal{E}_{2}=\left.\mathcal{L}_{\left(s a_{1}, a_{2}\right)}\right|_{X_{s a_{1}, a_{2}}}$ is defined and $\left.\mathcal{E}\right|_{X^{\prime}}=\iota^{\prime *} \mathcal{E}_{2}$. Hence we have a distinguished triangle $\left(\iota_{1}^{\prime} \mathcal{E}_{X^{\prime}}, \mathcal{E}_{2}, \mathcal{E}_{2}[-2](-1)\right)$ hence a distinguished triangle $\left(m_{!} j_{!}^{\prime}\left(\left.\mathcal{E}\right|_{X^{\prime}}, m_{!} \kappa_{!}^{\prime} \mathcal{E}_{2}, m_{!} \kappa_{!}^{\prime} \mathcal{E}_{2}[-2](-1)\right)\right.$. It follows that $A^{\prime} \dashv_{\Gamma}{ }^{p} H^{\cdot}\left(m_{!} \kappa_{!}^{\prime} \mathcal{E}_{2}\right)$. By the induction hypothesis there exists $e \in W$ such that $(b F)^{*}\left(e^{*} \mathcal{L}\right) \cong e^{*} \mathcal{L}$ and $A^{\prime} \dashv_{\Gamma}{ }^{p} H^{\cdot}\left(\left.m_{!}\left(e^{*} \mathcal{L}\right)_{b}\right|_{V}\right)$. This completes the proof of (a).

4.6. Let $\mathbf{t}=\left(J_{n}, w_{n}\right)_{n \geq 0} \in \mathcal{T}^{\prime}(J, \mathbf{c})$. Let $\mathcal{B}_{\mathbf{t}}=\left\{B^{\prime} \in \mathcal{B} ; P_{B^{\prime}, J} \in \mathcal{P}_{J}^{\mathbf{t}}\right\}$. For $a \in W$ let $\mathcal{B}_{\mathbf{t}, a}=\mathcal{B}_{\mathbf{t}} \cap \mathcal{B}_{a}$. Define $\xi_{\mathbf{t}, a}: \mathcal{B}_{\mathbf{t}, a} \rightarrow \mathcal{P}_{J}^{\mathbf{t}}$ by $B^{\prime} \mapsto P_{B^{\prime}, J}$. We show:

(a) Let $\mathcal{L} \in \mathcal{S}(T)^{a F}$. Let $A$ be a simple object of $\mathcal{M}_{\Gamma}\left(\mathcal{P}_{J}^{\mathrm{t}}\right)$ such that $A \dashv_{\Gamma}$ ${ }^{p} H^{\cdot}\left(\xi_{\mathbf{t}, a !}\left(\left.\mathcal{L}_{a}\right|_{\mathcal{B}_{\mathbf{t}, a}}\right)\right)$. Then there exist $b, e \in W$ such that $b^{*} e^{*} \mathcal{L} \cong e^{*} \mathcal{L}$ and $A \dashv_{\Gamma}$ ${ }^{p} H^{\cdot}\left(\vartheta^{*} \xi_{\mathbf{t}_{1}, b !}\left(\left.\left(e^{*} \mathcal{L}\right)_{b}\right|_{\mathcal{B}_{\mathbf{t}_{1}, b}}\right)\right)$.

Since $\xi_{\mathbf{t}, a !}\left(\left.\mathcal{L}_{a}\right|_{\mathcal{B}_{\mathbf{t}, a}}\right) \neq 0$ we have $\mathcal{B}_{\mathbf{t}, a} \neq \emptyset$. Thus there exists $B^{\prime} \in \mathcal{B}$ such that $\operatorname{pos}\left(B^{\prime}, F\left(B^{\prime}\right)\right)=a, P_{B^{\prime}, J} \in \mathcal{P}_{J}^{\mathrm{t}}$. We have $\operatorname{pos}\left(P_{B^{\prime}, J}, F\left(P_{B^{\prime}, J}\right)\right)=w_{0}$. Since $B^{\prime} \subset P_{B^{\prime}, J}, F\left(B^{\prime}\right) \subset F\left(P_{B^{\prime}, J}\right)$, it follows that $a \in W_{J} w_{0} W_{\mathbf{c}(J)}$ and $w_{0}=$ $\min \left(W_{J} a W_{\mathbf{c}(J)}\right)$.

Define $\phi: \mathcal{B}_{\mathbf{t}, a} \rightarrow \mathcal{B}_{\mathbf{t}_{1}}$ by $\phi\left(B^{\prime}\right)=\left(P_{B^{\prime}, J}\right)^{F\left(B^{\prime}\right)}$. (For $B^{\prime} \in \mathcal{B}_{\mathbf{t}}$ we have

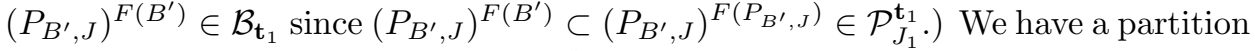
$\mathcal{B}_{\mathbf{t}_{1}}=\sqcup_{b \in W} \mathcal{B}_{\mathbf{t}_{1}, b}$. Setting $\mathcal{B}_{\mathbf{t}, a, b}=\phi^{-1}\left(\mathcal{B}_{\mathbf{t}_{1}, b}\right)$ we get a partition $\mathcal{B}_{\mathbf{t}, a}=\sqcup_{b \in W} \mathcal{B}_{\mathbf{t}, a, b}$. Let $\xi_{\mathbf{t}, a, b}: \mathcal{B}_{\mathbf{t}, a, b} \rightarrow \mathcal{P}_{J}^{\mathbf{t}}$ be the restriction of $\xi_{\mathbf{t}, a}$. By general principles we have $A \dashv_{\Gamma}{ }^{p} H^{\cdot}\left(\xi_{\mathbf{t}, a, b !}\left(\mathcal{L}_{a} \mid \mathcal{B}_{\mathbf{t}, a, b}\right)\right)$ for some $b \in W$. Let $\phi_{b}: \mathcal{B}_{\mathbf{t}, a, b} \rightarrow \mathcal{B}_{\mathbf{t}_{1}, b}$ be the restriction of $\phi$. We have $\vartheta \xi_{\mathbf{t}, a, b}=\xi_{\mathbf{t}_{1}, b} \phi_{b}$ (both compositions carry $B^{\prime}$ to $\left(P_{B^{\prime}, J}\right)^{F\left(P_{B^{\prime}, J}\right)}$.) Hence $\xi_{\mathbf{t}, a, b}=\vartheta^{-1} \xi_{\mathbf{t}_{1}, b} \phi_{b}$. Thus, $A \dashv_{\Gamma}{ }^{p} H^{\cdot}\left(\left(\vartheta^{-1}\right) \xi_{\mathbf{t}_{1}, b !} \phi_{b !}\left(\left.\mathcal{L}_{a}\right|_{\mathcal{B}_{\mathbf{t}, a, b}}\right)\right)$ and

$$
\vartheta_{!} A \dashv_{\Gamma}{ }^{p} H^{\cdot}\left(\xi_{\mathbf{t}_{1}, b !} \phi_{b !}\left(\left.\mathcal{L}_{a}\right|_{\mathcal{B}_{\mathbf{t}, a, b}}\right)\right) .
$$

We can write uniquely $a=a_{1} a_{2}$ where $a_{1} \in W_{J}, a_{2} \in{ }^{J} W$. We show that for any $\tilde{B} \in \mathcal{B}_{\mathbf{t}_{1}, b}$ we have

$$
\phi_{b}^{-1}(\tilde{B})=\left\{B^{\prime} \in \mathcal{B} ; \operatorname{pos}\left(B^{\prime}, \tilde{B}\right)=a_{1}, \operatorname{pos}\left(\tilde{B}, F\left(B^{\prime}\right)\right)=a_{2}\right\} .
$$

Assume first that $B^{\prime} \in \phi_{b}^{-1}(\tilde{B})$. We know that $\operatorname{pos}\left(B^{\prime}, F\left(B^{\prime}\right)\right)=a$. We have $\operatorname{pos}\left(B^{\prime},\left(P_{B^{\prime}, J}\right)^{F\left(B^{\prime}\right)}\right) \in W_{J}$ since $B^{\prime},\left(P_{B^{\prime}, J}\right)^{F\left(B^{\prime}\right)}$ are two Borel subgroups of $P_{B^{\prime}, J}$. From the definitions we have $\operatorname{pos}\left(\left(P_{B^{\prime}, J}\right){ }^{F\left(B^{\prime}\right)}, F\left(B^{\prime}\right)\right) \in{ }^{J} W$. We have automatically $\operatorname{pos}\left(B^{\prime},\left(P_{B^{\prime}, J}\right)^{F\left(B^{\prime}\right)}\right)=a_{1}, \operatorname{pos}\left(\left(P_{B^{\prime}, J}\right)^{F\left(B^{\prime}\right)}, F\left(B^{\prime}\right)\right)=a_{2}$ that is $\operatorname{pos}\left(B^{\prime}, \tilde{B}\right)=$ $a_{1}, \operatorname{pos}\left(\tilde{B}, F\left(B^{\prime}\right)\right)=a_{2}$.

Conversely, assume that $B^{\prime}$ belongs to the right hand side of (c). We have $l\left(a_{1} a_{2}\right)=l\left(a_{1}\right)+l\left(a_{2}\right)$ hence $\operatorname{pos}\left(B^{\prime}, F\left(B^{\prime}\right)\right)=a_{1} a_{2}=a$. Since the properties $\operatorname{pos}\left(B^{\prime}, \tilde{B}\right) \in W_{J}, \operatorname{pos}\left(\tilde{B}, F\left(B^{\prime}\right)\right) \in{ }^{J} W$ characterize $\tilde{B}$ and $\left(P_{B^{\prime}, J}\right)^{F\left(B^{\prime}\right)}$ has the same properties, it follows that $P^{F\left(B^{\prime}\right)}=\tilde{B}$ where $P=P_{B^{\prime}, J}$. Since $B^{\prime} \subset$ $P, F\left(B^{\prime}\right) \subset F(P)$ we have

$$
\operatorname{pos}(P, F(P))=\min \left(W_{J} \operatorname{pos}\left(B^{\prime}, F\left(B^{\prime}\right)\right) W_{\mathbf{c}(J)}\right)=\min \left(W_{J} a W_{\mathbf{c}(J)}\right)=w_{0} .
$$

It follows that $P^{F(P)} \in \mathcal{P}_{J \cap w_{0} \mathbf{c}(J) w_{0}^{-1}}=J_{1}$. Clearly, $\tilde{B}=P^{F\left(B^{\prime}\right)} \subset P^{F(P)}$. Hence $P^{F(P)}=P_{\tilde{B}, J_{1}} \in \mathcal{P}_{J_{1}}^{\mathbf{t}_{1}}$. It follows that $P \in \mathcal{P}_{J}^{\mathbf{t}}$. Thus, $B^{\prime} \in \mathcal{B}_{\mathbf{t}, a}$ and $\phi\left(B^{\prime}\right)=\tilde{B}$. Since $\tilde{B} \in \mathcal{B}_{\mathbf{t}_{1}, b}$, we see that $B^{\prime} \in \phi_{b}^{-1}(\tilde{B})$. This proves (c).

From (c) we see that $\left(B_{0}, B_{1}, B_{2}\right) \mapsto B_{0}$ is an isomorphism $X_{a_{1}, a_{2}} \rightarrow \mathcal{B}_{\mathbf{t}, a, b}$ where $X_{a_{1}, a_{2}}$ is defined as in 4.5(a) in terms of $V=\mathcal{B}_{\mathbf{t}_{1}, b}$. Under this isomorphism, $\phi_{b}$ 
corresponds to $X_{a_{1}, a_{2}} \rightarrow \mathcal{B}_{\mathbf{t}_{1}, b},\left(B_{0}, B_{1}, B_{2}\right) \mapsto B_{1}$. Applying $4.5\left(\right.$ a) with $V^{\prime}=\mathcal{P}_{J_{1}}^{\mathrm{t}_{1}}$, $m=\xi_{\mathbf{t}_{1}, b}, A^{\prime}=\vartheta_{!} A$ we see that there exists $e \in W$ such that $(b F)^{*}\left(e^{*} \mathcal{L}\right) \cong e^{*} \mathcal{L}$ and $\vartheta_{!} A \dashv_{\Gamma}{ }^{p} H^{\cdot}\left(\xi_{\mathbf{t}_{1}, b !}\left(\left.\left(e^{*} \mathcal{L}\right)_{b}\right|_{\mathcal{B}_{\mathbf{t}_{1}, b}}\right)\right)$. (The assumption of $4.5(\mathrm{a})$ is verified by (b).) Since $\vartheta$ is an isomorphism, it follows that $A \dashv_{\Gamma}{ }^{p} H^{\cdot}\left(\vartheta^{*} \xi_{\mathbf{t}_{1}, b !}\left(\left.\left(e^{*} \mathcal{L}\right)_{b}\right|_{\mathcal{B}_{\mathbf{t}_{1}, b}}\right)\right)$. This proves (a).

4.7. Let $\mathbf{t}=\left(J_{n}, w_{n}\right)_{n \geq 0} \in \mathcal{T}^{\prime}(J, \mathbf{c})$. Let $d=\operatorname{dim} \mathcal{P}_{J}^{\mathbf{t}}$. Let $a \in W$. We show:

(a) Let $\mathcal{L} \in \mathcal{S}(T)^{a \bar{F}}$. Let $A$ be a simple object of $\mathcal{M}_{\Gamma}\left(\mathcal{P}_{J}^{\mathbf{t}}\right)$ such that $A \dashv_{\Gamma}$ ${ }^{p} H^{\cdot}\left(\xi_{\mathbf{t}, a !}\left(\left.\mathcal{L}_{a}\right|_{\mathcal{B}_{\mathbf{t}, a}}\right)\right)$. Then $A \in \mathbb{S}\left(\mathcal{P}_{J}^{\mathbf{t}}\right)$.

More generally, we show that (a) holds when $J, \mathbf{t}$ are replaced by $J_{n}, \mathbf{t}_{n}, n \geq 0$. First we show:

(b) If the result holds for $n=1$, then it holds for $n=0$.

Let $A$ be as in (a). By 4.6(a) there exist $b, e \in W$ such that $(b F)^{*}\left(e^{*} \mathcal{L}\right) \cong e^{*} \mathcal{L}$ and $A \dashv_{\Gamma}{ }^{p} H^{*}\left(\vartheta^{*} \xi_{\mathbf{t}_{1}, b !}\left(\left.\left(e^{*} \mathcal{L}\right)_{b}\right|_{\mathcal{B}_{\mathbf{t}_{1}, b}}\right)\right)$. Since $\vartheta$ is an isomorphism, there exists a simple object $A^{\prime}$ of $\mathcal{M}_{\Gamma}\left(\mathcal{P}_{J_{1}}^{\mathbf{t}_{1}}\right)$ such that $A=\vartheta^{*} A^{\prime}$. From our assumption we have $A^{\prime} \dashv_{\Gamma}{ }^{p} H^{\cdot}\left(\xi_{\mathbf{t}_{1}, b !}\left(\left.\left(e^{*} \mathcal{L}\right)_{b}\right|_{\mathcal{B}_{\mathbf{t}_{1}, b}}\right)\right)$. Since (b) holds for $n=1$ we have $A^{\prime} \cong \underline{M}[d]$ for some irreducible $L_{J_{\infty}}^{F^{\prime}}$-module $M$. Hence $A$ is of the same form. Thus (b) holds.

Similarly, if the result holds for some $n \geq 1$, then it holds for $n-1$. In this way we see that it suffices to prove the result for $n$ large. Thus in the remainder of this proof we assume, as we may, that $J_{0}=J_{1}=\cdots=J$ and $w_{0}=w_{1}=\cdots=w$. We can write uniquely $a=a_{1} a_{2}$ where $a_{1} \in W_{J}, a_{2} \in{ }^{J} W$. Since $\xi_{\mathbf{t}, a !}\left(\left.\mathcal{L}_{a}\right|_{\mathcal{B}_{\mathbf{t}, a}}\right) \neq 0$, we have $\mathcal{B}_{\mathbf{t}, a} \neq \emptyset$. From this we deduce as in the proof of $4.6\left(\right.$ a) that $a \in W_{J} w W_{\mathbf{c}(J)}$. Since $w \mathbf{c}(J) w^{-1}=J$ we must have $w W_{\mathbf{c}(J)}=W_{J} w$ so that $a \in W_{J} w$. Since $w \in{ }^{J} W$ (see 4.2(b)) it follows that $w=a_{2}$. In particular, we have $a_{2} \in{ }^{J} W^{\mathbf{c}(J)}$. Hence if $B^{\prime \prime} \in \mathcal{B}_{a}$, then $\operatorname{pos}\left(P_{B^{\prime \prime}, J}, F\left(P_{B^{\prime \prime}, J}\right)\right)=a_{2}=w$. Since in our case $\mathcal{P}_{J}^{\mathbf{t}}=$ $\left\{P \in \mathcal{P}_{J} ; \operatorname{pos}(P, F(P))=w\right\}$ (see 4.2(e)), we see that $P_{B^{\prime \prime}, J} \in \mathcal{P}_{J}^{\mathrm{t}}$. Thus we have $\mathcal{B}_{a}=\mathcal{B}_{\mathbf{t}, a}$ and $A \dashv_{\Gamma}{ }^{p} H^{\cdot}\left(\xi_{\mathbf{t}, a !}\left(\mathcal{L}_{a}\right)\right)$. Let $\tilde{\mathcal{B}}_{a}=\left\{g U \in G / U ; g^{-1} F(g) \in U \dot{a} U\right\}$. Recall from 4.3(b) that $f_{a}: \tilde{\mathcal{B}}_{a} \rightarrow \mathcal{B}_{a}, g U \mapsto g B g^{-1}$ is a finite principal covering with group $\mathfrak{T}=\left\{t \in T ; \dot{a} F(t) \dot{a}^{-1}=t\right\}$. From 2.5 we see that $\mathcal{L}_{a}$ is a direct summand of $f_{a !} \overline{\mathbf{Q}}_{l}$. Thus we have $A \dashv_{\Gamma}{ }^{p} H^{\cdot}\left(\xi_{\mathbf{t}, a !} f_{a !} \overline{\mathbf{Q}}_{l}\right)$.

By 4.3(a), $L_{J}=\dot{w} L_{\mathbf{c}(J)} \dot{w}^{-1}$ is a common Levi subgroup of $P_{J}, \dot{w} P_{\mathbf{c}(J)} \dot{w}^{-1}$. Let $F^{\prime}: L_{J} \rightarrow L_{J}$ be as in 4.3 . Let $\Lambda=L_{J}^{F^{\prime}}$. Let $\mathcal{B}^{\prime}$ be the variety of Borel subgroups of $L_{J}$. For $\beta, \beta^{\prime}$ in $\mathcal{B}^{\prime}$ we have $\operatorname{pos}\left(\beta U_{P_{J}}, \beta^{\prime} U_{P_{J}}\right)=y$ for a unique $y \in W_{J}$; we then also write $\operatorname{pos}^{\prime}\left(\beta, \beta^{\prime}\right)=y$. Let $Y^{\prime}=\left\{\beta \in \mathcal{B}^{\prime} ; \operatorname{pos}^{\prime}\left(\beta, F^{\prime}(\beta)\right)=a_{1}\right\}$. Now $\Lambda$ acts on $Y^{\prime}$ by conjugation. Let $\tilde{\mathcal{P}}_{J}^{\mathrm{t}}$ be as in 4.3 . Note that $\Lambda$ acts (freely) on $\tilde{\mathcal{P}}_{J}^{\mathrm{t}} \times Y^{\prime}$ by $l:\left(g U_{P_{J}}, \beta\right) \mapsto\left(g l^{-1} U_{P_{J}}, l \beta l^{-1}\right)$ and we can form the orbit space $\Lambda \backslash\left(\tilde{\mathcal{P}}_{J}^{\mathrm{t}} \times Y^{\prime}\right)$.

We define $\psi: \tilde{\mathcal{P}}_{J}^{\mathrm{t}} \times Y^{\prime} \rightarrow \mathcal{B}_{a}$ by $\left(g U_{P_{J}}, \beta\right) \mapsto g \beta U_{P_{J}} g^{-1}$. We show that $\psi$ is well defined; that is, $\operatorname{pos}\left(g \beta U_{P_{J}} g^{-1}, F(g) F(\beta) U_{P_{\mathbf{c}(J)}} F\left(g^{-1}\right)\right)=a$ for $g U_{P_{J}} \in \tilde{\mathcal{P}}_{J}^{\mathrm{t}}$. Since $a=a_{1} w, l(a)=l\left(a_{1}\right)+l(w)$, it is enough to show that for $\left(g U_{P_{J}}, \beta\right) \in \tilde{\mathcal{P}}_{J}^{\mathrm{t}} \times Y^{\prime}$ we have

(c) $\operatorname{pos}\left(g \beta U_{P_{J}} g^{-1}, g F^{\prime}(\beta) U_{P_{J}} g^{-1}\right)=a_{1}$,

(d) $\operatorname{pos}\left(g F^{\prime}(\beta) U_{P_{J}} g^{-1}, F(g) F(\beta) U_{P_{\mathbf{c}(J)}} F\left(g^{-1}\right)\right)=w$.

Now the left hand side of (c) is equal to $\operatorname{pos}\left(\beta U_{P_{J}}, F^{\prime}(\beta) U_{P_{J}}\right)=\operatorname{pos}^{\prime}\left(\beta, F^{\prime}(\beta)\right)=$ $a_{1}$, proving (c). We have $g^{-1} F(g)=u \dot{w} u^{\prime}$ where $u \in U_{P_{J}}, u^{\prime} \in U_{P_{\mathbf{c}(J)}}$. The left 
hand side of $(\mathrm{d})$ is equal to

$$
\begin{aligned}
& \operatorname{pos}\left(F^{\prime}(\beta) U_{P_{J}}, g^{-1} F(g) F(\beta) U_{P_{\mathbf{c}(J)}} F\left(g^{-1}\right) g\right) \\
& =\operatorname{pos}\left(F^{\prime}(\beta) U_{P_{J}}, u \dot{w} u^{\prime} F(\beta) U_{P_{\mathbf{c}(J)}} u^{\prime-1} \dot{w}^{-1} u^{-1}\right) \\
& =\operatorname{pos}\left(F^{\prime}(\beta) U_{P_{J}}, \dot{w} F(\beta) U_{P_{\mathbf{c}(J)}} \dot{w}^{-1}\right) \\
& =\operatorname{pos}\left(F^{\prime}(\beta) U_{P_{J}}, F^{\prime}(\beta) U_{\dot{w} P_{\mathbf{c}(J)}} \dot{w}^{-1}\right)=\operatorname{pos}\left(P_{J}, \dot{w} P_{\mathbf{c}(J)} \dot{w}^{-1}\right) .
\end{aligned}
$$

(The last equality follows from L5, [II, 8.3].) This equals $w$.

We see that $\psi$ is well defined. We show:

(e) The map $\bar{\psi}: \Lambda \backslash\left(\tilde{\mathcal{P}}_{J}^{\mathrm{t}} \times Y^{\prime}\right) \rightarrow \mathcal{B}_{a}$ induced by $\psi$ is an isomorphism.

Let $B^{\prime} \in \mathcal{B}_{a}$. We can find uniquely $\tilde{B} \in \mathcal{B}$ such that $\operatorname{pos}\left(B^{\prime}, \tilde{B}\right)=a_{1}, \operatorname{pos}\left(\tilde{B}, F\left(B^{\prime}\right)\right)$ $=w$. Then $P_{B^{\prime}, J}=P_{\tilde{B}, J}$ and $\operatorname{pos}\left(P_{\tilde{B}, J}, P_{F\left(B^{\prime}\right), \mathbf{c}(J)}\right)=w$. Hence $\operatorname{pos}\left(P_{B^{\prime}, J}, F\left(P_{B^{\prime}, J}\right)\right)$ $=w$; that is, $P_{B^{\prime}, J} \in \mathcal{P}_{J}^{\mathrm{t}}$. Since $\tilde{\mathcal{P}}_{J}^{\mathrm{t}} \rightarrow \mathcal{P}_{J}^{\mathrm{t}}$ is surjective (see 4.3) we can find $g U_{P_{J}} \in \tilde{\mathcal{P}}_{J}^{\mathrm{t}}$ such that $g P_{J} g^{-1}=P_{B^{\prime}, J}$; note that $g U_{P_{J}}$ is unique up to the action of $\Lambda$. Since $B^{\prime} \subset P_{B^{\prime}, J}$ we have $g^{-1} B^{\prime} g \subset P_{J}$ hence there is a unique $\beta \in \mathcal{B}^{\prime}$ such that $g^{-1} B^{\prime} g=\beta U_{P_{J}}$. As above we see that

$$
\operatorname{pos}\left(g F^{\prime}(\beta) U_{P_{J}} g^{-1}, F(g) F(\beta) U_{P_{\mathbf{c}(J)}} F\left(g^{-1}\right)\right)=w ;
$$

that is, $\operatorname{pos}\left(g F^{\prime}(\beta) U_{P_{J}} g^{-1}, F\left(B^{\prime}\right)\right)=w$. Thus $g F^{\prime}(\beta) U_{P_{J}} g^{-1}$ is a Borel subgroup of $P_{B^{\prime}, J}$ whose relative position with $F\left(B^{\prime}\right)$ is $w$. But there is only one such Borel subgroup. Therefore $g F^{\prime}(\beta) U_{P_{J}} g^{-1}=\tilde{B}$. Since $\operatorname{pos}\left(B^{\prime}, \tilde{B}\right)=a_{1}$ we have $\operatorname{pos}\left(g \beta U_{P_{J}} g^{-1}, g F^{\prime}(\beta) U_{P_{J}} g^{-1}\right)=a_{1}$ hence $\operatorname{pos}\left(\beta U_{P_{J}}, F^{\prime}(\beta) U_{P_{J}}\right)=a_{1}$; that is, $\operatorname{pos}^{\prime}\left(\beta, F^{\prime}(\beta)\right)=a_{1}$. Thus to $B^{\prime} \in \mathcal{B}_{a}$ we have associated $\left(g U_{P_{J}}, \beta\right) \in \tilde{\mathcal{P}}_{J}^{\mathrm{t}} \times Y^{\prime}$; its $\Lambda$-orbit is well defined. Thus we have a well defined map $\mathcal{B}_{a} \rightarrow \Lambda \backslash\left(\tilde{\mathcal{P}}_{J}^{\mathrm{t}} \times Y^{\prime}\right)$. Clearly, this is the inverse of $\bar{\psi}$. This proves (e).

Now let $\beta^{\prime}=B \cap L_{J}$, and let $U^{\prime}$ be the unipotent radical of $\beta^{\prime}$. Let $U^{\prime \prime}=$ $U^{\prime} \cap F^{\prime-1}\left(\dot{a}_{1}^{-1} U^{\prime} \dot{a}_{1}\right)$. Let $\tilde{Y}^{\prime}=\left\{l U^{\prime \prime} \in L_{J} / U^{\prime \prime} ; l^{-1} F^{\prime}(l) \in U^{\prime} \dot{a}_{1}\right\}$. As in 4.3(b),(c) the map $\rho: \tilde{Y}^{\prime} \rightarrow Y^{\prime}, l U^{\prime \prime} \mapsto l \beta^{\prime} l^{-1}$ is a finite principal covering with group $\left\{t \in T ; \dot{a}_{1} F^{\prime}(t) \dot{a}_{1}^{-1}=t\right\}=\mathfrak{T}$. Now $\Lambda$ acts freely on $\tilde{\mathcal{P}}_{J}^{\mathrm{t}} \times \tilde{Y}^{\prime}$ by

$$
l_{0}:\left(g U_{P_{J}}, l U^{\prime \prime}\right) \mapsto\left(g l_{0}^{-1} U_{P_{J}}, l_{0} l U^{\prime \prime}\right)
$$

and we can form the orbit space $\Lambda \backslash\left(\tilde{\mathcal{P}}_{J}^{\mathrm{t}} \times \tilde{Y}^{\prime}\right)$.

The map $\tilde{\mathcal{P}}_{J}^{\mathrm{t}} \times \tilde{Y}^{\prime} \rightarrow \tilde{\mathcal{B}}_{a},\left(g U_{P_{J}}, l U^{\prime \prime}\right) \mapsto g l U$ induces a map $\tilde{\psi}: \Lambda \backslash\left(\tilde{\mathcal{P}}_{J}^{\mathrm{t}} \times \tilde{Y}^{\prime}\right) \rightarrow \tilde{\mathcal{B}}_{a}$ which is easily seen to be an isomorphism. We have a commutative diagram

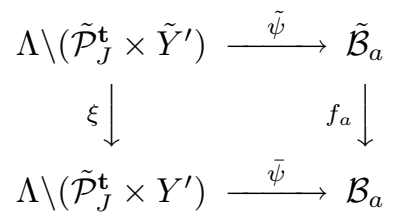

where $\xi$ is induced by $\left(g U_{P_{J}}, l U^{\prime \prime}\right) \mapsto\left(g U_{P_{J}}, l \beta^{\prime} l^{-1}\right)$ (a principal $\mathfrak{T}$-bundle). Note that the horizontal maps in this diagram are isomorphisms.

Under the isomorphism (e), the map $\xi_{\mathbf{t}, a}$ becomes the map $\xi^{\prime}: \Lambda \backslash\left(\tilde{\mathcal{P}}_{J}^{\mathrm{t}} \times Y^{\prime}\right) \rightarrow$ $\mathcal{P}_{J}^{\mathbf{t}}$ induced by $\left(g U_{P_{J}}, \beta\right) \mapsto g P_{J} g^{-1}$. It follows that $\xi_{\mathbf{t}, a !} f_{a !} \overline{\mathbf{Q}}_{l}=\left(\xi^{\prime} \xi\right) ! \overline{\mathbf{Q}}_{l}$ and $A \dashv_{\Gamma}{ }^{p} H^{\cdot}\left(\left(\xi^{\prime} \xi\right) ! \overline{\mathbf{Q}}_{l}\right)$. We extend the natural $\Gamma$-actions on $\mathcal{P}_{J}^{\mathbf{t}}$ and on $\Lambda \backslash\left(\tilde{\mathcal{P}}_{J}^{\mathbf{t}} \times \tilde{Y}^{\prime}\right)$ to $\Gamma \times \Lambda$-actions with $\Lambda$ acting trivially. Then $A,{ }^{p} H^{j}\left(\left(\xi^{\prime} \xi\right) ! \overline{\mathbf{Q}}_{l}\right)$ are naturally objects of $\mathcal{M}_{\Gamma \times \Lambda}\left(\mathcal{P}_{J}^{\mathbf{t}}\right)$ and $A \dashv_{\Gamma \times \Lambda}{ }^{p} H^{\cdot}\left(\left(\xi^{\prime} \xi\right) ! \overline{\mathbf{Q}}_{l}\right)$. Let $f: \tilde{\mathcal{P}}_{J}^{\mathbf{t}} \rightarrow \mathcal{P}_{J}^{\mathbf{t}}$ be as in 4.3. Now $\Gamma \times \Lambda$ acts on $\tilde{\mathcal{P}}_{J}^{\mathrm{t}}$ as in 4.4 (compatibly with $f$ ). Moreover, $f^{*} A, f^{*}\left({ }^{p} H^{j}\left(\left(\xi^{\prime} \xi\right) ! \overline{\mathbf{Q}}_{l}\right)\right)=$ 
${ }^{p} H^{j}\left(f^{*}\left(\xi^{\prime} \xi\right) ! \overline{\mathbf{Q}}_{l}\right)$ are objects of $\mathcal{M}_{\Gamma \times \Lambda}\left(\tilde{\mathcal{P}}_{J}^{\mathbf{t}}\right)$ and $f^{*} A \dashv_{\Gamma \times \Lambda}{ }^{p} H^{\cdot}\left(f^{*}\left(\xi^{\prime} \xi\right) ! \overline{\mathbf{Q}}_{l}\right)$. Let $p_{1}: \tilde{\mathcal{P}}_{J}^{\mathrm{t}} \times \tilde{Y}^{\prime} \rightarrow \tilde{\mathcal{P}}_{J}^{\mathrm{t}}$ be the first projection. Now $\Gamma \times \Lambda$ acts on $\tilde{\mathcal{P}}_{J}^{\mathrm{t}} \times \tilde{Y}^{\prime}$ by $\left(g_{0}, l_{0}\right):$ $\left(g U_{J}, l U^{\prime \prime}\right) \mapsto\left(g_{0} g l_{0}^{-1} U_{J}, l_{0} l U^{\prime \prime}\right)$ and $p_{1}$ is compatible with the $\Gamma \times \Lambda$-actions. We have $f^{*}\left(\xi^{\prime} \xi\right)_{!} \overline{\mathbf{Q}}_{l}=p_{1 !} \overline{\mathbf{Q}}_{l}$. We see that $f^{*} A \dashv_{\Gamma \times \Lambda}{ }^{p} H^{*}\left(p_{1 !} \overline{\mathbf{Q}}_{l}\right)$. Let $p_{2}: \tilde{\mathcal{P}}_{J}^{\mathrm{t}} \times \tilde{Y}^{\prime} \rightarrow \tilde{Y}^{\prime}$ be the second projection. Now $\Gamma \times \Lambda$ acts on $\tilde{Y}^{\prime}$ by $\left(g_{0}, l_{0}\right): l U^{\prime \prime} \mapsto l_{0} l U^{\prime \prime}$ and $p_{2}$ is compatible with the $\Gamma \times \Lambda$-actions. The obvious maps $\tilde{\mathcal{P}}_{J}^{\mathrm{t}} \stackrel{e}{\rightarrow}$ point $\stackrel{e^{\prime}}{\leftarrow} \tilde{Y}^{\prime}$ are again compatible with the $\Gamma \times \Lambda$-actions (the action on the point is trivial). We have $p_{1} \overline{\mathbf{Q}}_{l}=e^{*} e_{!}^{\prime} \overline{\mathbf{Q}}_{l}$ hence $f^{*} A \dashv_{\Gamma \times \Lambda}{ }^{p} H^{*}\left(e^{*} e_{!}^{\prime} \overline{\mathbf{Q}}_{l}\right)$. We have a spectral sequence in $\mathcal{M}_{\Gamma \times \Lambda}\left(\tilde{\mathcal{P}}_{J}^{\mathrm{t}}\right)$ with $E_{2}={ }^{p} H^{\cdot}\left(e^{* p} H^{\cdot}\left(e_{!}^{\prime} \overline{\mathbf{Q}}_{l}\right)\right)$ and $E_{\infty}$ is an associated graded of ${ }^{p} H^{*}\left(e^{*} e_{!}^{\prime} \overline{\mathbf{Q}}_{l}\right)$. We have $f^{*} A \dashv_{\Gamma \times \Lambda} E_{\infty}$ hence $f^{*} A \dashv_{\Gamma \times \Lambda} E_{2}$. Now ${ }^{p} H^{*}\left(e_{!}^{\prime} \overline{\mathbf{Q}}_{l}\right)$ is just a $\Gamma \times \Lambda$-module $M$ with trivial action of $\Gamma$ and $e^{* p} H^{*}\left(e_{1}^{\prime} \overline{\mathbf{Q}}_{l}\right)$ is the local system $f^{*} \underline{M}$ (notation of 4.4). Since $\tilde{\mathcal{P}}_{J}^{\mathrm{t}}$ is smooth of pure dimension $d,{ }^{p} H^{j}\left(e^{* p} H^{\cdot}\left(e_{!}^{\prime} \overline{\mathbf{Q}}_{l}\right)\right)$ is 0 if $j \neq d$ and is $f^{*} \underline{M}[d]$ if $j=d$. Thus $E_{2}=f^{*} \underline{M}[d]$. We see that $f^{*} A \dashv_{\Gamma \times \Lambda} f^{*}(\underline{M})[d]$. It follows that $A \dashv_{\Gamma} \underline{M}[d]$. This implies (a).

4.8. Let $\mathbf{t}=\left(J_{n}, w_{n}\right)_{n \geq 0} \in \mathcal{T}^{\prime}(J, \mathbf{c})$. Let $d=\operatorname{dim} \mathcal{P}_{J}^{\mathbf{t}}$. Let $\mathcal{L} \in \mathcal{S}(T)$ and $\mathbf{s}$ be as in 2.6. Let $\overline{\mathcal{L}}$ be the local system on $\mathcal{Z}^{\mathbf{s}}$ as in 2.11. Let $\Upsilon^{\mathbf{s}}: \mathcal{Z}^{\mathbf{s}} \rightarrow \mathcal{P}_{J}, \bar{\Upsilon}^{\mathbf{s}}: \bar{Z}^{\mathbf{s}} \rightarrow \mathcal{P}_{J}$ be as in 3.2. We show:

(a) Let $A$ be a simple object of $\mathcal{M}_{\Gamma}\left(\mathcal{P}_{J}\right)$ such that $A \dashv_{\Gamma}{ }^{p} H^{\cdot}\left(\Upsilon_{!}^{\mathbf{s}} \overline{\mathcal{L}}\right)$. Let $h: \mathcal{P}_{J}^{\mathrm{t}} \rightarrow$ $\mathcal{P}_{J}$ be the inclusion. Let $A^{\prime}$ be a simple object of $\mathcal{M}_{\Gamma}\left(\mathcal{P}_{J}^{\mathbf{t}}\right)$ such that $A^{\prime} \dashv_{\Gamma}{ }^{p} H^{\cdot}\left(h^{*} A\right)$. Then $A^{\prime} \cong \underline{M}[d]$ for some irreducible $L_{J_{\infty}}^{F^{\prime}}$-module $M$.

Let $K=\bar{\Upsilon}_{!}^{\mathbf{s}} \overline{\mathcal{L}}^{\sharp}$. Using 2.13(a) we see that $\Upsilon_{!}^{\mathbf{s}} \overline{\mathcal{L}}=K$. Hence $A \dashv_{\Gamma}{ }^{p} H^{\cdot}(K)$. We have a spectral sequence in $\mathcal{M}_{\Gamma}\left(\mathcal{P}_{J}^{\mathrm{t}}\right)$ with $E_{2}={ }^{p} H^{\cdot}\left(h^{*}\left({ }^{p} H^{\cdot} K\right)\right)$ and $E_{\infty}$ is an associated graded of ${ }^{p} H^{\cdot}\left(h^{*} K\right)$. Since $\Upsilon^{\mathbf{s}}$ is proper, we see from the decomposition theorem [BBD that $K \cong \oplus_{i}^{p} H^{i}(K)[-i]$ and that each ${ }^{p} H^{i}(K)$ is semisimple as an object of $\mathcal{M}\left(\mathcal{P}_{J}\right)$ hence also as an object of $\mathcal{M}_{\Gamma}\left(\mathcal{P}_{J}\right)$. It also follows that $h^{*} K \cong \oplus_{i} h^{*}\left({ }^{p} H^{i}(K)\right)[-i]$ hence ${ }^{p} H^{j}\left(h^{*} K\right) \cong \oplus_{i}{ }^{p} H^{j-i}\left(h^{*}\left({ }^{p} H^{i}(K)\right)\right)$. This shows that $E_{2} \cong E_{\infty}$ as objects of $\mathcal{M}\left(\mathcal{P}_{J}^{\mathrm{t}}\right)$. Thus the spectral sequence above is degenerate when regarded in $\mathcal{M}\left(\mathcal{P}_{J}^{\mathrm{t}}\right)$. But then it is also degenerate in $\mathcal{M}_{\Gamma}\left(\mathcal{P}_{J}^{\mathrm{t}}\right)$. Using $A^{\prime} \dashv_{\Gamma}$ ${ }^{p} H^{\cdot}\left(h^{*} A\right)$ and the fact that $A$ is a direct summand of ${ }^{p} H^{\cdot}(K)\left(\right.$ in $\left.\mathcal{M}_{\Gamma}\left(\mathcal{P}_{J}\right)\right)$ we see that $A^{\prime} \dashv_{\Gamma} E_{2}$. It follows that $A^{\prime} \dashv_{\Gamma} E_{\infty}$; that is, $A^{\prime} \dashv_{\Gamma}{ }^{p} H^{\cdot}\left(h^{*} \Upsilon_{!}^{\mathrm{s}} \overline{\mathcal{L}}\right)$. As in the proof of the implication $3.2(\mathrm{v}) \Longrightarrow 3.2(\mathrm{i})$ we deduce that $A^{\prime} \dashv_{\Gamma}{ }^{p} H^{\cdot}\left(h^{*} \Pi_{!}^{(a)} \mathcal{L}_{(a)}\right)$ $\left(\Pi^{(a)}\right.$ as in 3.2) for some $a \in W$. Now (a) follows from 4.7(a).

4.9. Let $A$ be a simple object of $\mathcal{M}_{\Gamma}\left(\mathcal{P}_{J}\right)$ such that $A \dashv_{\Gamma}{ }^{p} H^{\cdot}\left(\Upsilon_{!}^{\mathrm{s}} \overline{\mathcal{L}}\right)$ with $\mathcal{L}$, s as in 2.6. We show:

(a) There exists $\mathbf{t}=\left(J_{n}, w_{n}\right)_{n \geq 0} \in \mathcal{T}^{\prime}(J, \mathbf{c})$ and an irreducible $L_{J_{\infty}}^{F^{\prime}}$-module $M$ such that $A \cong \underline{M}^{\sharp}[d]$ where $\underline{M}^{\sharp}$ is as in 4.4 and $d=\operatorname{dim} \mathcal{P}_{J}^{\mathrm{t}}$.

Since $\mathcal{P}_{J}=\cup_{\mathbf{t} \in \mathcal{T}^{\prime}(J, \mathbf{c})} \mathcal{P}_{J}^{\mathbf{t}}$, we can find $\mathbf{t} \in \mathcal{T}^{\prime}(J, \mathbf{c})$ such that $\operatorname{supp}(A) \cap \mathcal{P}_{J}^{\mathbf{t}}$ is open dense in $\operatorname{supp}(A)$. Then, denoting by $h: \mathcal{P}_{J}^{\mathrm{t}} \rightarrow \mathcal{P}_{J}$ the inclusion, we see that $h^{*} A$ is a simple object of $\mathcal{M}_{\Gamma}\left(\mathcal{P}_{J}^{\mathrm{t}}\right)$. As in $4.8(\mathrm{a})$, we have $h^{*} A \cong \underline{M}[d]$ for some irreducible $L_{J_{\infty}}^{F^{\prime}}$-module $M$. It follows that $A$ is of the required form.

4.10. Let $\mathbf{t}=\left(J_{n}, w_{n}\right)_{n \geq 0} \in \mathcal{T}^{\prime}(J, \mathbf{c})$. We set $w=w_{\infty} \in{ }^{J} W$. We show:

(a) for any $b \in W_{J_{\infty}}, B^{\prime} \mapsto P_{B^{\prime}, J}$ is a well defined map $\mathcal{B}_{b w} \rightarrow \mathcal{P}_{J}^{\mathrm{t}}$;

(b) for $b=1$, the map $\mathcal{B}_{w} \rightarrow \mathcal{P}_{J}^{\mathrm{t}}$ in (a) is surjective.

We prove (a). Let $B^{\prime} \in \mathcal{B}_{b w}$. Let $P=P_{B^{\prime}, J}$. By $4.2(\mathrm{f})$ we have $\operatorname{pos}(P, F(P))=$ $\min \left(W_{J} w W_{\mathbf{c}(J)}\right)=w_{0}$. Define $P^{n}$ in terms of $P$ as in 4.1. We have $P^{1}=P^{F(P)} \in$ 
$\mathcal{P}_{J_{1}}$. As in the proof of 4.6(c) (with $\left.a_{1}=b, a_{2}=w\right)$ we see that $b=\operatorname{pos}\left(B^{\prime}, P^{F\left(B^{\prime}\right)}\right)$, $w=\operatorname{pos}\left(P^{F\left(B^{\prime}\right)}, F\left(B^{\prime}\right)\right)$. Since $b \in W_{J_{1}}$ we have $\operatorname{pos}\left(B^{\prime}, P^{F\left(B^{\prime}\right)}\right) \in W_{J_{1}}$ hence $B^{\prime} \subset P^{1}$. From the definitions we have $w_{1}=\min \left(W_{J_{1}} w W_{\mathbf{c}\left(J_{1}\right)}\right)$ hence

$$
\operatorname{pos}\left(P^{1}, F\left(P^{1}\right)\right)=\min \left(W_{J_{1}} \operatorname{pos}\left(B^{\prime}, F\left(B^{\prime}\right)\right) W_{\mathbf{c}\left(J_{1}\right)}\right)=\min \left(W_{J_{1}} b w W_{\mathbf{c}\left(J_{1}\right)}\right)=w_{1} .
$$

By the same argument applied to $B^{\prime}, P^{1}, \mathbf{t}_{1}$ instead of $B^{\prime}, P, \mathbf{t}$ we see that $P^{2} \in \mathcal{P}_{J_{2}}$ and $\operatorname{pos}\left(P^{2}, F\left(P^{2}\right)\right)=w_{2}$. (We have $w \in{ }^{J_{1}} W$ since $J_{1} \subset J$.) Continuing in this way we see that $P^{n} \in \mathcal{P}_{J_{n}}$ and $\operatorname{pos}\left(P^{n}, F\left(P^{n}\right)\right)=w_{n}$ for all $n \geq 0$. Thus $P \in \mathcal{P}_{J}^{\mathrm{t}}$. This proves (a).

We prove (b). Let $P \in \mathcal{P}_{J}^{\mathrm{t}}$. Define $P^{\infty}$ in terms of $P$ as in 4.1. We have $\operatorname{pos}\left(P^{\infty}, F\left(P^{\infty}\right)\right)=w$. Hence

$$
\begin{aligned}
p r_{2}: & \left\{\left(B^{\prime}, B^{\prime \prime}\right) \in \mathcal{B} \times \mathcal{B} ; B^{\prime} \subset P^{\infty}, B^{\prime \prime} \subset F\left(P^{\infty}\right), \operatorname{pos}\left(B^{\prime}, B^{\prime \prime}\right)=w\right\} \\
& \rightarrow\left\{B^{\prime \prime} \in \mathcal{B} ; B^{\prime \prime} \subset F\left(P^{\infty}\right)\right\}
\end{aligned}
$$

is a bijection with inverse $B^{\prime \prime} \mapsto\left(\left(P^{\infty}\right)^{B^{\prime \prime}}, B^{\prime \prime}\right)$. The condition that $\left(B^{\prime}, B^{\prime \prime}\right)$ in the domain of $p r_{2}$ satisfies $B^{\prime \prime}=F\left(B^{\prime}\right)$ is that $B^{\prime \prime}$ is a fixed point of the map $B^{\prime \prime} \mapsto F\left(\left(P^{\infty}\right)^{B^{\prime \prime}}\right)$ of the flag manifold of $F\left(P^{\infty}\right)$ into itself. This map may be identified with the map induced by $F^{\prime}: L_{J} \rightarrow L_{J}$ (see 4.3) on the flag manifold of $L_{J}$ hence it has at least one fixed point. Thus there exist $\left(B^{\prime}, B^{\prime \prime}\right) \in \mathcal{B} \times \mathcal{B}$ such that $B^{\prime \prime}=F\left(B^{\prime}\right), B^{\prime} \subset P^{\infty}, \operatorname{pos}\left(B^{\prime}, B^{\prime \prime}\right)=w$. Then $B^{\prime} \in \mathcal{B}_{a}$ and $B^{\prime} \subset P$ (since $\left.P^{\infty} \subset P\right)$. This proves (b).

4.11. Let $\mathbf{t}=\left(J_{n}, w_{n}\right)_{n \geq 0} \in \mathcal{T}^{\prime}(J, \mathbf{c})$. We set $w=w_{\infty} \in{ }^{J} W$. We show:

(a) Let $b \in W_{J_{\infty}}$. Let $\mathcal{L} \in \mathcal{S}(T)^{\text {bw } F}$. Let $A$ be a simple object of $\mathcal{M}_{\Gamma}\left(\mathcal{P}_{J}^{\mathbf{t}_{1}}\right)$ such that $A \dashv_{\Gamma}{ }^{p} H^{\cdot}\left(\xi_{\mathbf{t}_{1}, w \mathbf{c}(b) !}\left(b^{*} \mathcal{L}\right)_{w \mathbf{c}(b)}\right)$. Then $\vartheta^{*} A \dashv_{\Gamma}{ }^{p} H^{\cdot}\left(\xi_{\mathbf{t}, b w !} \mathcal{L}_{b w}\right)$.

The result makes sense since $\mathcal{B}_{\mathbf{t}, b w}=\mathcal{B}_{b w}$ by $4.10(\mathrm{a})$ and $\mathcal{B}_{\mathbf{t}_{1}, w \mathbf{c}(b)}=\mathcal{B}_{w \mathbf{c}(b)}$ (this follows from 4.10(a) applied to $\mathbf{t}_{1}, w, w \mathbf{c}(b) w^{-1}$ instead of $\mathbf{t}, w, b$; note that $w \mathbf{c}\left(J_{\infty}\right) w^{-1}=J_{\infty}$ by $4.2(\mathrm{e})$ hence $\left.w \mathbf{c}(b) w^{-1} \in W_{J_{\infty}}\right)$. This shows also that $l(w \mathbf{c}(b))=l(w)+l(\mathbf{c}(b))$. (Since $w \in{ }^{J} W$ we have $l\left(\left(w \mathbf{c}(b) w^{-1}\right) w\right)=l\left(w \mathbf{c}(b) w^{-1}\right)+$ $l(w)$.) We define $h: \mathcal{B}_{b w} \rightarrow \mathcal{B}_{w \mathbf{c}(b)}$ by $B^{\prime} \mapsto B^{\prime \prime}$ with $B^{\prime \prime}$ defined by $\operatorname{pos}\left(B^{\prime}, B^{\prime \prime}\right)=$ $b, \operatorname{pos}\left(B^{\prime \prime}, F\left(B^{\prime}\right)\right)=w$. This is an isomorphism whose inverse $\mathcal{B}_{w \mathbf{c}(b)} \rightarrow \mathcal{B}_{b w}$ is given by $B^{\prime \prime} \mapsto B^{\prime}$ with $B^{\prime}$ defined by $\operatorname{pos}\left(B^{\prime}, B^{\prime \prime}\right)=b, \operatorname{pos}\left(B^{\prime \prime}, F\left(B^{\prime}\right)\right)=w$. We have a commutative diagram

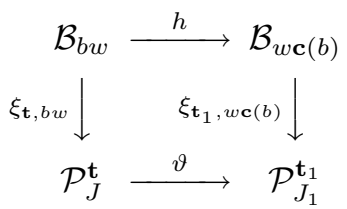

where the horizontal maps are isomorphism. From the definitions we see that $h^{*}\left(\left(b^{*} \mathcal{L}\right)_{w \mathbf{c}(b)}\right)=\mathcal{L}_{b w}$. The result follows.

4.12. Let $\mathbf{t}=\left(J_{n}, w_{n}\right)_{n \geq 0} \in \mathcal{T}^{\prime}(J, \mathbf{c})$. We set $w=w_{\infty} \in{ }^{J} W$. Let $d=\operatorname{dim} \mathcal{P}_{J}^{\mathbf{t}}$. Let $\Lambda=L_{J_{\infty}}^{F^{\prime}}$. Let $M$ be a finite dimensional irreducible $\Lambda$-module over $\overline{\mathbf{Q}}_{l}$. Let $\underline{M}[d] \in \mathcal{M}_{\Gamma}\left(\mathcal{P}_{J}^{\mathbf{t}}\right)$ be as in $4.4(\mathrm{c})$. We show:

(a) there exists $b \in W_{J_{\infty}}$ and $\mathcal{L} \in \mathcal{S}(T)^{b w F}$ such that $\underline{M}[d] \dashv_{\Gamma}{ }^{p} H \cdot\left(\xi_{\mathbf{t}, b w !} \mathcal{L}_{b w}\right)$;

(b) there exists $b \in W_{J_{\infty}}$ and $\mathcal{L} \in \mathcal{S}(T)^{b w F}$ such that $\underline{M}^{\sharp}[d] \dashv_{\Gamma}{ }^{p} H^{\cdot}\left(\Pi_{!}^{(b w)} \mathcal{L}_{(b w)}\right)$.

We prove (a). More generally, we show that for any $n \geq 0$ :

(c) there exists $b_{n} \in W_{J_{\infty}}$ and $\mathcal{L} \in \mathcal{S}(T)^{b_{n} w F}$ such that $\underline{M}[d]$ (regarded as an object of $\left.\mathcal{M}_{\Gamma}\left(\mathcal{P}_{J_{n}}^{\mathbf{t}_{n}}\right)\right)$ satisfies $\underline{M}[d] \dashv_{\Gamma}{ }^{p} H^{\cdot}\left(\xi_{\mathbf{t}_{n}, b_{n} w !} \mathcal{L}_{b_{n} w}\right)$. 
If (c) holds for $n=1$, then, by 4.11(a) it holds for $n=0$. Similarly, if (c) holds for some $n \geq 1$, then it holds for $n-1$. Hence it is enough to prove (c) for large $n$. Thus we may assume that $J_{0}=J_{1}=\cdots=J$ and $w_{0}=w_{1}=\cdots=w$. By [DL, 7.7] applied to $\Lambda$, there exists $a_{1} \in W_{J_{\infty}}$ such that $M \dashv_{\Lambda}{ }^{p} H^{\cdot}\left(e_{!}^{\prime} \overline{\mathbf{Q}}_{l}\right)$ (in $\mathcal{M}_{\Lambda}$ ( point)). (The reference to DL could be replaced by a self-contained proof, see 7.9.) By definition, $f^{*} \underline{M}[d]=e^{*} M[d]$ with $f$ as in 4.3, $e$ as in 4.7. We have $e^{*} M[d] \dashv_{\Gamma \times \Lambda} e^{*}\left({ }^{p} H^{*}\left(e_{!}^{\prime} \overline{\mathbf{Q}}_{l}\right)\right)[d]$ hence $f^{*} \underline{M}[d] \dashv_{\Gamma \times \Lambda} e^{*}\left({ }^{p} H^{*}\left(e_{!}^{\prime} \overline{\mathbf{Q}}_{l}\right)\right)[d]$ in $\mathcal{M}_{\Gamma \times \Lambda}\left(\tilde{\mathcal{P}}_{J}^{\mathbf{t}}\right)$. Since $\tilde{\mathcal{P}}_{J}^{\mathbf{t}}$ is smooth of pure dimension $d$ we have $e^{*}\left({ }^{p} H^{i}\left(e_{!}^{\prime} \overline{\mathbf{Q}}_{l}\right)\right)[d]={ }^{p} H^{i+d}\left(e^{*} e_{!}^{\prime} \overline{\mathbf{Q}}_{l}\right)$. Since

$$
e^{*} e_{!}^{\prime} \overline{\mathbf{Q}}_{l}=p_{1 !} \overline{\mathbf{Q}}_{l}=f^{*}\left(\xi^{\prime} \xi\right) ! \overline{\mathbf{Q}}_{l}=f^{*} \xi_{\mathbf{t}, a !} f_{a !} \overline{\mathbf{Q}}_{l}
$$

(notation of 4.7 with $\left.a=a_{1} w\right)$ we have $f^{*} \underline{M}[d] \dashv_{\Gamma \times \Lambda}{ }^{p} H^{\cdot}\left(f^{*} \xi_{\mathbf{t}, a !} f_{a !} \overline{\mathbf{Q}}_{l}\right)$. Since $f$ is a finite principal covering we also have $f^{*} M[d] \dashv_{\Gamma \times \Lambda} f^{*}\left({ }^{p} H^{\cdot}\left(\xi_{\mathbf{t}, a !} f_{a !} \overline{\mathbf{Q}}_{l}\right)\right)$. It follows that $\underline{M}[d] \dashv_{\Gamma}{ }^{p} H^{\cdot}\left(\xi_{\mathbf{t}, a !} f_{a !} \overline{\mathbf{Q}}_{l}\right)$. Now $f_{a !} \overline{\mathbf{Q}}_{l}=\oplus_{\mathcal{L}} \mathcal{L}_{a}$ where $\mathcal{L}$ runs over the local systems in $\mathcal{S}(T)^{a F}$ (up to isomorphism). Hence for some such $\mathcal{L}$ we have $\underline{M}[d] \dashv_{\Gamma}^{p} H^{\cdot}\left(\xi_{\mathbf{t}, a !} \mathcal{L}_{a}\right)$. This proves $(\mathrm{a})$.

We prove (b). Let $b, \mathcal{L}$ be such that (a) holds. Let $K=\Pi_{!}^{(b w)} \mathcal{L}_{(b w)}, K^{\prime}=$ $\xi_{\mathrm{t}, b w !} \mathcal{L}_{b w}$. We have $\underline{M}[d] \dashv_{\Gamma}{ }^{p} H^{\cdot}\left(K^{\prime}\right)$. Let $\kappa: \mathcal{P}_{J}^{\mathbf{t}} \rightarrow \mathcal{P}_{J}$ be the inclusion. We have $K=\kappa_{!} K^{\prime}$. Let $C$ be the closure of $\mathcal{P}_{J}^{\mathrm{t}}$ in $\mathcal{P}_{J}$. Let $\kappa^{\prime}: \mathcal{P}_{J}^{\mathrm{t}} \rightarrow C, \kappa^{\prime \prime}: C \rightarrow \mathcal{P}_{J}$ be the inclusions. Let $\tilde{M}=I C(C, \underline{M})[d]$. Let $K_{1}=\kappa_{!}^{\prime} K$. We show:

$$
\tilde{M} \dashv_{\Gamma}{ }^{p} H^{\cdot}\left(K_{1}\right) .
$$

We have $\kappa^{* *} K_{1}=K^{\prime}$ and $\kappa^{*}\left({ }^{p} H^{j}\left(K_{1}\right)\right)={ }^{p} H^{j}\left(K^{\prime}\right)$. Let $0=X_{0} \subset X_{1} \subset \ldots \subset$ $X_{m}={ }^{p} H^{j}\left(K_{1}\right)$ be a composition series of ${ }^{p} H^{j}\left(K_{1}\right)$ in $\mathcal{M}_{\Gamma}(C)$. Aplying $\kappa^{\prime *}$ to the exact sequence $0 \rightarrow X_{i-1} \rightarrow X_{i} \rightarrow X_{i} / X_{i-1} \rightarrow 0$ (where $1 \leq i \leq m$ ) we get an exact sequence $0 \rightarrow \kappa^{*}\left(X_{i-1}\right) \rightarrow \kappa^{*}\left(X_{i}\right) \rightarrow \kappa^{\prime *}\left(X_{i} / X_{i-1}\right) \rightarrow 0$ in $\mathcal{M}_{\Gamma}\left(\mathcal{P}_{\Gamma}^{\mathbf{t}}\right)$. Since $X_{i} / X_{i-1}$ is simple and $\mathcal{P}_{\Gamma}^{\mathrm{t}}$ is open dense in $C$ we see that $\kappa^{* *}\left(X_{i} / X_{i-1}\right)$ is either simple or 0 . It follows that any composition factor of $\kappa^{\prime *}\left(X_{m}\right)$ is isomorphic to $\kappa^{\prime *}\left(X_{i} / X_{i-1}\right)$ for some $i$. In particular, $\underline{M}[d] \cong \kappa^{\prime *}\left(X_{i} / X_{i-1}\right)$ for some $i$. It follows that $\tilde{M} \cong X_{i} / X_{i-1}$ for some $i$. Thus (d) holds.

Applying $\kappa_{!}^{\prime \prime}$ to $(\mathrm{d})$ we obtain

$$
\underline{M}^{\sharp}[d]=\kappa_{!}^{\prime \prime} \tilde{M} \dashv_{\Gamma} \kappa_{!}^{\prime \prime p} H^{\cdot}\left(K_{1}\right)={ }^{p} H^{\cdot}\left(\kappa_{!}^{\prime \prime} K_{1}\right)={ }^{p} H^{\cdot}(K) .
$$

This proves (b).

Theorem 4.13. Let $K$ be a simple object of $\mathcal{M}_{\Gamma}\left(\mathcal{P}_{J}\right)$. Then $K \in \mathbb{S}^{\prime}\left(\mathcal{P}_{J}\right)$ (see 3.7) if and only if $K \in \mathbb{S}\left(\mathcal{P}_{J}\right)$.

If $K \in \mathbb{S}\left(\mathcal{P}_{J}\right)$, then by $4.12(\mathrm{~b})$ it satisfies $3.2(\mathrm{i})$. If $K$ satisfies $3.2(\mathrm{v})$, then by $4.9(\mathrm{a}), K \in \mathbb{S}\left(\mathcal{P}_{J}\right)$. This completes the proof.

Theorem 4.14. Let $\mathbf{t}=\left(J_{n}, w_{n}\right)_{n \geq 0} \in \mathcal{T}^{\prime}(J, \mathbf{c})$. Let $h: \mathcal{P}_{J}^{\mathbf{t}} \rightarrow \mathcal{P}_{J}$ be the inclusion. Let $A \in \mathbb{S}\left(\mathcal{P}_{J}\right)$. Let $A^{\prime}$ be a simple object of $\mathcal{M}_{\Gamma}\left(\mathcal{P}_{J}^{\mathbf{t}}\right)$ such that $A^{\prime} \dashv_{\Gamma}{ }^{p} H^{*}\left(h^{*} A\right)$. Then $A^{\prime} \in \mathbb{S}\left(\mathcal{P}_{J}^{\mathrm{t}}\right)$.

By assumption, $A$ is as in 4.8(a). The result now follows from 4.8(a).

\section{Some computations in the Weyl GRoup}

5.1. Let $\mathcal{L} \in \mathcal{S}(T)$. Define $\tilde{l}: W \rightarrow \mathbf{N}$ by $\tilde{l}(w)=\left|\left(\alpha \in R_{\mathcal{L}}^{+} ; w(\alpha) \in R^{-}\right\}\right|$. Let $\mathbf{s}=\left(s_{1}, \ldots s_{r}\right)$ be a sequence in $\mathbf{I} \cup\{1\}$. Let

$$
\mathcal{I}_{\mathbf{s}}=\left\{i \in[1, r] ; s_{i} \neq 1, s_{1} s_{2} \ldots s_{i} \ldots s_{2} s_{1} \in W_{\mathcal{L}}\right\}
$$


This agrees with the definition in 2.4 .

Lemma 5.2. We have $\left|\mathcal{I}_{\mathbf{s}}\right| \geq \tilde{l}\left(s_{r} \ldots s_{1}\right)$ with equality if $l\left(s_{r} \ldots s_{1}\right)=l\left(s_{1}\right)+\cdots+$ $l\left(s_{r}\right)$.

$$
\begin{aligned}
& \text { Let } \\
& \qquad X=\left\{\alpha \in R_{\mathcal{L}}^{+} ;\left(s_{r} \ldots s_{1}\right)(\alpha) \in R^{-}\right\}, \\
& \qquad X^{\prime}=\left\{\alpha \in R_{\mathcal{L}}^{+} ; \alpha=s_{1} s_{2} \ldots s_{i-1}\left(\alpha_{s_{i}}\right) \text { for some } i \in[1, r] \text { such that } s_{i} \neq 1\right\} .
\end{aligned}
$$

We have $X \subset X^{\prime}$. We have $|X|=\tilde{l}\left(s_{r} \ldots s_{1}\right)$ hence $\tilde{l}\left(s_{r} \ldots s_{1}\right) \leq\left|X^{\prime}\right|$. Define $f: \mathcal{I}_{\mathbf{s}} \rightarrow R_{\mathcal{L}}$ by $f(i)=s_{1} s_{2} \ldots s_{i-1}\left(\alpha_{s_{i}}\right)$; then $X^{\prime}=f\left(\mathcal{I}_{\mathbf{s}}\right) \cap R_{\mathcal{L}}^{+}$. Hence $\left|X^{\prime}\right| \leq$ $\left|f\left(\mathcal{I}_{\mathbf{s}}\right)\right| \leq\left|\mathcal{I}_{\mathbf{s}}\right|$ and the desired inequality is proved. Assume now that $l\left(s_{r} \ldots s_{1}\right)=$ $l\left(s_{1}\right)+\cdots+l\left(s_{r}\right)$. Then $s_{1} s_{2} \ldots s_{i-1}\left(\alpha_{s_{i}}\right)\left(i \in[1, r], s_{i} \neq 1\right)$ are distinct in $R^{+}$. Hence for $i \in \mathcal{I}_{\mathbf{s}}, s_{1} s_{2} \ldots s_{i-1}\left(\alpha_{s_{i}}\right)$ are distinct elements of $X$. Thus $\left|\mathcal{I}_{\mathbf{s}}\right| \leq|X|$. It follows that $\left|\mathcal{I}_{\mathbf{s}}\right|=|X|$.

Lemma 5.3. Let $j \in \mathcal{I}_{\mathbf{s}}$. Let $\mathbf{s}(j)=\left(s_{1}^{\prime}, s_{2}^{\prime}, \ldots, s_{r}^{\prime}\right)$ with $s_{i}^{\prime}=s_{i}$ for $i \neq j, s_{j}^{\prime}=1$. We have $\mathcal{I}_{\mathbf{s}(j)}=\mathcal{I}_{\mathbf{s}}-\{j\}$.

Let $h \in \mathcal{I}_{\mathbf{s}}-\{j\}$. We have $s_{1} s_{2} \ldots s_{h} \ldots s_{2} s_{1} \in W_{\mathcal{L}}$. Hence if $j<h$, we have

$$
\begin{aligned}
& s_{1} s_{2} \ldots \hat{s}_{j} \ldots s_{h} \ldots \hat{s}_{j} \ldots s_{2} s_{1} \\
& \quad=\left(s_{1} s_{2} \ldots s_{j} \ldots s_{2} s_{1}\right)\left(s_{1} s_{2} \ldots s_{h} \ldots s_{2} s_{1}\right)\left(s_{1} s_{2} \ldots s_{j} \ldots s_{2} s_{1}\right) \in W_{\mathcal{L}},
\end{aligned}
$$

so that $h \in \mathcal{I}_{\mathbf{s}(j)}$. ('denotes an omitted symbol.) If $j>h$, then $h \in \mathcal{I}_{\mathbf{s}(j)}$ is obvious.

Conversely, assume that $h \in \mathcal{I}_{\mathbf{s}(j)}$. Clearly, $h \neq j$. Assume first that $j<h$. We have

$$
\begin{aligned}
& s_{1} s_{2} \ldots s_{h} \ldots s_{2} s_{1} \\
& =\left(s_{1} s_{2} \ldots s_{j} \ldots s_{2} s_{1}\right)\left(s_{1} s_{2} \ldots \hat{s}_{j} \ldots s_{h} \ldots \hat{s}_{j} \ldots s_{2} s_{1}\right)\left(s_{1} s_{2} \ldots s_{j} \ldots s_{2} s_{1}\right) \in W_{\mathcal{L}} .
\end{aligned}
$$

Hence $h \in \mathcal{I}_{\mathbf{s}}$. If $j>h$, then it is clear that $h \in \mathcal{I}_{\mathbf{s}}$. The lemma is proved.

Lemma 5.4. Let $h$ be the smallest element of $\mathcal{I}_{\mathbf{s}}$. Then $s_{1} s_{2} \ldots s_{h} \ldots s_{2} s_{1} \in \mathbf{I}_{\mathcal{L}}$.

Let $\mathbf{s}^{\prime}=\left(s_{1}, s_{2}, \ldots, s_{h}, \ldots, s_{2}, s_{1}\right)$. We have

$s_{1} s_{2} \ldots s_{h} \ldots s_{2} s_{1} \in W_{\mathcal{L}}$

$s_{1} s_{2} \ldots s_{h-1} \ldots s_{2} s_{1} \notin W_{\mathcal{L}}$ or $s_{h-1}=1$,

$s_{1} s_{2} \ldots s_{h-2} \ldots s_{2} s_{1} \notin W_{\mathcal{L}}$ or $s_{h-2}=1, \ldots$

Hence the middle term in $\mathbf{s}^{\prime}$ has index in $\mathcal{I}_{\mathbf{s}^{\prime}}$ but all terms preceding it have index not in $\mathcal{I}_{\mathbf{s}^{\prime}}$. We show that the term in $\mathbf{s}^{\prime}$ immediately following the middle term has index not in $\mathcal{I}_{\mathbf{s}^{\prime}}$. Otherwise it would be $\neq 1$ and

$$
s_{1} s_{2} \ldots s_{h-1} s_{h} s_{h-1} s_{h} s_{h-1} \ldots s_{2} s_{1} \notin W_{\mathcal{L}} .
$$

Multiplying on the left and right by $s_{1} s_{2} \ldots s_{h} \ldots s_{2} s_{1}$ we find

$$
s_{1} s_{2} \ldots s_{h-1} \ldots s_{2} s_{1} \in W_{\mathcal{L}},
$$

a contradiction. Similarly we see that all terms in $\mathbf{s}^{\prime}$ following the middle term have index not in $\mathcal{I}_{\mathbf{s}^{\prime}}$. Thus $\left|\mathcal{I}_{\mathbf{s}^{\prime}}\right|=1$. By Lemma 5.2 we have

$$
\tilde{l}\left(s_{1} s_{2} \ldots s_{h} \ldots s_{2} s_{1}\right) \leq 1 \text {. }
$$

Since $s_{1} s_{2} \ldots s_{h} \ldots s_{2} s_{1} \in W_{\mathcal{L}}-\{1\}$, it follows that $s_{1} s_{2} \ldots s_{h} \ldots s_{2} s_{1} \in \mathbf{I}_{\mathcal{L}}$. The lemma is proved. 
5.5. We write the elements of $\mathcal{I}_{\mathbf{s}}$ in ascending order: $i_{1}<i_{2}<\cdots<i_{b}$. Define a sequence $\mathbf{S}=\left(S_{1}, S_{2}, \ldots, S_{b}\right)$ in $W$ by

$$
\begin{aligned}
& S_{1}=s_{1} s_{2} \ldots s_{i_{1}} \ldots s_{2} s_{1} \\
& S_{2}=s_{1} s_{2} \ldots \hat{s}_{i_{1}} \ldots s_{i_{2}} \ldots \hat{s}_{i_{1}} \ldots s_{2} s_{1}, \ldots \\
& S_{b}=s_{1} s_{2} \ldots \hat{s}_{i_{1}} \ldots \hat{s}_{i_{2}} \ldots \hat{s}_{i_{b-1}} \ldots s_{i_{b}} \ldots \hat{s}_{i_{b-1}} \ldots \hat{s}_{i_{2}} \ldots \hat{s}_{i_{1}} \ldots s_{2} s_{1}, \\
& \omega=s_{1} \ldots \hat{s}_{i_{1}} \ldots \hat{s}_{i_{2}} \ldots \hat{s}_{i_{b}} \ldots s_{r} .
\end{aligned}
$$

Lemma 5.6. (a) $S_{1}, S_{2}, \ldots, S_{b}$ belong to $\mathbf{I}_{\mathcal{L}}$.

(b) $\tilde{l}\left(\omega^{-1}\right)=0$.

(c) $s_{1} s_{2} \ldots s_{r}=S_{1} S_{2} \ldots S_{b} \omega$.

We use induction on $b$. Assume first that $b=0$. By 5.2 we have $\tilde{l}\left(s_{r} \ldots s_{1}\right)=0$ that is $\tilde{l}\left(\omega^{-1}\right)=0$ and the lemma is clear. Assume now that $b \geq 1$ and that the lemma holds for $b-1$. Let $h=i_{1}$. By 5.3 we have $\mathcal{I}_{\mathbf{s}(h)}=\mathcal{I}_{\mathbf{s}}-\{h\}$. By the induction hypothesis, $S_{2}, S_{3}, \ldots, S_{b}$ belong to $\mathbf{I}_{\mathcal{L}}, s_{1} \ldots \hat{s}_{i_{1}} \ldots s_{r}=S_{2} S_{3} \ldots S_{b} \omega$, $\tilde{l}\left(\omega^{-1}\right)=0$. By 5.4 we have $S_{1} \in \mathbf{I}_{\mathcal{L}}$. It follows that

$$
s_{1} s_{2} \ldots s_{r}=\left(s_{1} \ldots s_{i_{1}} \ldots s_{1}\right)\left(s_{1} \ldots \hat{s}_{i_{1}} \ldots s_{r}\right)=S_{1} S_{2} \ldots S_{b} \omega .
$$

The lemma is proved.

5.7. Let $W_{\mathcal{L}}^{\prime}=\left\{w \in W ; w^{*} \mathcal{L} \cong \mathcal{L}\right\}$, a subgroup of $W$. Let $W^{\prime 0} \mathcal{L}=\{w \in$ $\left.W_{\mathcal{L}}^{\prime} ; w\left(R_{\mathcal{L}}^{+}\right)=R_{\mathcal{L}}^{+}\right\}$, a subgroup of $W_{\mathcal{L}}^{\prime}$. Note that $W_{\mathcal{L}}^{\prime}=W^{\prime 0}{ }_{\mathcal{L}} W_{\mathcal{L}}$ (semidirect product with $W_{\mathcal{L}}$ normal).

In the remainder of this section we fix an automorphism $c$ of finite order of $T$ that induces a permutation of $R$, one of $\check{R}$ and one of $R^{+}$.

Lemma 5.8. Assume that $F_{0}^{*}\left(s_{1} s_{2} \ldots s_{r} c\right)^{*} \mathcal{L} \cong \mathcal{L}$. Let $c^{\prime}=\omega c$ so that $s_{1} s_{2} \ldots s_{r} c$ $=S_{1} S_{2} \ldots S_{b} c^{\prime}$ and $F_{0}^{*}\left(S_{1} S_{2} \ldots S_{b} c^{\prime}\right)^{*} \mathcal{L} \cong \mathcal{L}$. Then $c^{\prime}$ is an automorphism of finite order of $T$ that induces a permutation of $R_{\mathcal{L}}$, one of $\check{R}_{\mathcal{L}}$ and one of $R_{\mathcal{L}}^{+}$.

We first show:

$$
c^{\prime}\left(R_{\mathcal{L}}\right)=R_{\mathcal{L}}
$$

It is enough to show that for any coroot $\kappa: \mathbf{k}^{*} \rightarrow T$ such that $\kappa^{*}(\mathcal{L}) \cong \overline{\mathbf{Q}}_{l}$ we have $\left(c^{\prime} \kappa\right)^{*} \mathcal{L} \cong \overline{\mathbf{Q}}_{l}$. From $F_{0}^{*}\left(S_{1} S_{2} \ldots S_{b} c^{\prime}\right)^{*} \mathcal{L} \cong \mathcal{L}$ and $S_{1} S_{2} \ldots S_{r} \in W_{\mathcal{L}}$ we deduce $F_{0}^{*} c^{\prime *} \mathcal{L} \cong \mathcal{L}$. Hence $\kappa^{*} F_{0}^{*} c^{\prime *} \mathcal{L} \cong \kappa^{*} \mathcal{L} \cong \overline{\mathbf{Q}}_{l}$. Now $F_{0} \kappa=\kappa F_{0}^{\prime}$ where $F_{0}^{\prime}: \mathbf{k}^{*} \rightarrow \mathbf{k}^{*}$ is $x \mapsto x^{q}$. Hence $F_{0}^{\prime *} \kappa^{*} c^{\prime *} \mathcal{L}=\overline{\mathbf{Q}}_{l}=F_{0}^{\prime *} \overline{\mathbf{Q}}_{l}$ and $\kappa^{*} c^{\prime *} \mathcal{L}=\overline{\mathbf{Q}}_{l}$. This proves (i).

By 5.6(b) we have $\omega^{-1}\left(R_{\mathcal{L}}^{+}\right) \subset R^{+}$. Moreover, $c^{-1}\left(R^{+}\right)=R^{+}$hence $c^{-1} \omega^{-1}\left(R_{\mathcal{L}}^{+}\right)$ $\subset R^{+}$. Using (i) we see that $c^{\prime-1}\left(R_{\mathcal{L}}^{+}\right) \subset R_{\mathcal{L}}^{+}$hence $c^{\prime}\left(R_{\mathcal{L}}^{+}\right)=R_{\mathcal{L}}^{+}$. The lemma is proved.

5.9. Let $\tilde{\mathbf{s}}=\left(\tilde{s}_{1}, \tilde{s}_{2}, \ldots, \tilde{s}_{\tilde{r}}\right)$ be a second sequence in $\mathbf{I}$. Then the subset $\mathcal{I}_{\tilde{\mathbf{s}}}$ is defined in terms of $\tilde{\mathbf{s}}, \mathcal{L}$ in the same way as $\mathcal{I}_{\mathbf{s}}$ is defined in terms of $\mathbf{s}, \mathcal{L}$.

We write the elements of $\mathcal{I}_{\tilde{\mathbf{s}}}$ in ascending order: $j_{1}<j_{2}<\cdots<j_{\tilde{b}}$. Define $\tilde{\mathbf{S}}=\left(\tilde{S}_{1}, \tilde{S}_{2}, \ldots, \tilde{S}_{\tilde{b}}\right), \tilde{\omega}$ in terms of $\tilde{\mathbf{s}}, \mathcal{L}$ in the same way that $\mathbf{S}, \omega$ are defined in 5.5 in terms of $\mathbf{s}, \mathcal{L}$. As in 5.6 we have $\tilde{S}_{i} \in \mathbf{I}_{\mathcal{L}}$ for $i \in[1, \tilde{b}]$ and $\tilde{s}_{1} \tilde{s}_{2} \ldots \tilde{s}_{\tilde{r}}=\tilde{S}_{1} \tilde{S}_{2} \ldots \tilde{S}_{\tilde{b}} \tilde{\omega}$. We assume that

$$
F_{0}^{*}([\mathbf{s}] c)^{*} \mathcal{L} \cong \mathcal{L}, F_{0}^{*}([\tilde{\mathbf{s}}] c)^{*} \mathcal{L} \cong \mathcal{L} .
$$

From 5.8 we see that $\omega c$ and $\tilde{\omega} c$ are automorphisms of finite order of $T$ that induce a permutation of $R_{\mathcal{L}}$, one of $\check{R}_{\mathcal{L}}$ and one of $R_{\mathcal{L}}^{+}$. Moreover, we have

$$
F_{0}^{*}\left(S_{1} S_{2} \ldots S_{b} \omega c\right)^{*} \mathcal{L} \cong \mathcal{L}, F_{0}^{*}\left(\tilde{S}_{1} \tilde{S}_{2} \ldots \tilde{S}_{\tilde{b}} \tilde{\omega} c\right)^{*} \mathcal{L} \cong \mathcal{L},
$$


or equivalently $F_{0}^{*}(\omega c)^{*} \mathcal{L} \cong \mathcal{L}, F_{0}^{*}(\tilde{\omega} c)^{*} \mathcal{L} \cong \mathcal{L}$. Let

$$
\mathfrak{F}=\left\{f \in W_{\mathcal{L}}^{\prime 0} ; f^{-1} \omega c f=\tilde{\omega} c\right\} .
$$

Now for $f \in \mathfrak{F}$ we have $f \mathbf{I}_{\mathcal{L}} f^{-1}=\mathbf{I}_{\mathcal{L}}$; we set

$$
{ }^{f} \tilde{\mathbf{S}}=\left(f \tilde{S}_{1} f^{-1}, f \tilde{S}_{2} f^{-1}, \ldots f \tilde{S}_{\tilde{b}} f^{-1}\right),
$$

a sequence in $\mathbf{I}_{\mathcal{L}}$. Since $F_{0}^{*}(\omega c)^{*} \mathcal{L} \cong \mathcal{L}$, we have

$$
F_{0}^{*}\left(\left(f \tilde{S}_{1} f^{-1}\right)\left(f \tilde{S}_{2} f^{-1}\right) \ldots\left(f \tilde{S}_{\tilde{b}} f^{-1}\right) \omega c\right)^{*} \mathcal{L} \cong \mathcal{L} .
$$

We set $\rho=r+\tilde{r}$. Let $\mathcal{A}(W, c, \mathcal{L}, \mathbf{s}, \tilde{\mathbf{s}})$ be the set of all sequences $\left(a_{0}, a_{1}, \ldots, a_{\rho}\right)$ in $W$ such that

$$
\begin{aligned}
& a_{j-1}^{-1} a_{j} \in\left\{1, \tilde{s}_{j}\right\} \text { for } j \in \mathcal{I}_{\tilde{\mathbf{s}}} ; \\
& a_{j-1}^{-1} a_{j}=\tilde{s}_{j} \text { for } j \in[1, \tilde{r}]-\mathcal{I}_{\tilde{\mathbf{s}}} ; \\
& a_{\tilde{r}+i} a_{\tilde{r}+i-1}^{-1} \in\left\{1, s_{i}\right\} \text { for } i \in \mathcal{I}_{\mathbf{s}} ; \\
& a_{\tilde{r}+i} a_{\tilde{r}+i-1}^{-1}=s_{i} \text { for } i \in[1, r]-\mathcal{I}_{\mathbf{s}} ; \\
& a_{\rho}=c a_{0} c^{-1} ; \\
& a_{0}^{*} \mathcal{L} \cong \mathcal{L} .
\end{aligned}
$$

Replacing here $W, c, \mathcal{L}, \mathbf{s}, \tilde{\mathbf{s}}$ by $W_{\mathcal{L}}, \omega c, \overline{\mathbf{Q}}_{l}, \mathbf{S},{ }^{f} \tilde{\mathbf{S}}$, we obtain for any $f \in \mathfrak{F}$ a set $\mathcal{A}\left(W_{\mathcal{L}}, \omega c, \overline{\mathbf{Q}}_{l}, \mathbf{S},{ }^{f} \tilde{\mathbf{S}}\right)$. From the definition, $\mathcal{A}\left(W_{\mathcal{L}}, \omega c, \overline{\mathbf{Q}}_{l}, \mathbf{S},{ }^{f} \tilde{\mathbf{S}}\right)$ consists of all sequences $\left(A_{0}, A_{1}, \ldots, A_{b+\tilde{b}}\right)$ in $W_{\mathcal{L}}$ such that

$A_{j-1}^{-1} A_{j} \in\left\{1, f \tilde{S}_{j} f^{-1}\right\}$ for $j \in[1, \tilde{b}]$;

$A_{\tilde{b}+i} A_{\tilde{b}+i-1}^{-1} \in\left\{1, S_{i}\right\}$ for $i \in[1, b]$;

$A_{b+\tilde{b}}=(\omega c) A_{0}(\omega c)^{-1}$.

We now state the following result.

Proposition 5.10. There is a canonical bijection

$$
\Psi: \mathcal{A}(W, c, \mathcal{L}, \mathbf{s}, \tilde{\mathbf{s}}) \stackrel{\sim}{\rightarrow} \sqcup_{f \in \mathcal{F}} \mathcal{A}\left(W_{\mathcal{L}}, \omega c, \overline{\mathbf{Q}}_{l}, \mathbf{S},{ }^{f} \tilde{\mathbf{S}}\right) .
$$

Let $\left(a_{0}, a_{1}, \ldots, a_{\rho}\right) \in \mathcal{A}(W, c, \mathcal{L}, \mathbf{s}, \tilde{\mathbf{s}})$. Consider the product

$$
\begin{aligned}
& a_{\tilde{r}}\left(a_{\tilde{r}+i_{1}}^{-1} a_{\tilde{r}+i_{1}-1}\right) a_{\tilde{r}}^{-1}=\left(a_{\tilde{r}} a_{\tilde{r}+1}^{-1}\right)\left(a_{\tilde{r}+1} \alpha_{\tilde{r}+2}^{-1}\right) \ldots\left(a_{\tilde{r}+i_{1}-2} a_{\tilde{r}+i_{1}-1}^{-1}\right) \\
& \quad \times\left(a_{\tilde{r}+i_{1}-1} a_{\tilde{r}+i_{1}}^{-1}\right)\left(a_{\tilde{r}+i_{1}-1} a_{\tilde{r}+i_{1}-2}^{-1}\right) \ldots\left(a_{\tilde{r}+2} a_{\tilde{r}+1}^{-1}\right)\left(a_{\tilde{r}+1} a_{\tilde{r}}^{-1}\right) .
\end{aligned}
$$

The right hand side is equal to $s_{1} s_{2} \ldots s_{i_{1}-1} x s_{i_{1}-1} \ldots s_{2} s_{1}$ where $x$ is either $s_{i_{1}}$ or

1. Hence it is equal to $s_{1} s_{2} \ldots s_{i_{1}-1} s_{i_{1}} s_{i_{1}-1} \ldots s_{2} s_{1}$ or to 1 . Thus we have

$$
a_{\tilde{r}}\left(a_{\tilde{r}+i_{1}}^{-1} a_{\tilde{r}+i_{1}-1}\right) a_{\tilde{r}}^{-1} \in\left\{S_{1}, 1\right\} .
$$

Similarly,

$$
\begin{aligned}
& a_{\tilde{r}}\left(a_{\tilde{r}+i_{1}-1}^{-1} a_{\tilde{r}+i_{1}}\right)\left(a_{\tilde{r}+i_{2}-1}^{-1} a_{\tilde{r}+i_{2}}\right) \ldots\left(a_{\tilde{r}+i_{e-1}-1}^{-1} a_{\tilde{r}+i_{e-1}}\right)\left(a_{\tilde{r}+i_{e}}^{-1} a_{\tilde{r}+i_{e}-1}\right) \\
& \quad \times\left(a_{\tilde{r}+i_{e-1}}^{-1} a_{\tilde{r}+i_{e-1}-1}\right) \ldots\left(a_{\tilde{r}+i_{2}}^{-1} a_{\tilde{r}+i_{2}-1}\right)\left(a_{\tilde{r}+i_{1}}^{-1} a_{\tilde{r}+i_{1}-1}\right) a_{\tilde{r}}^{-1} \in\left\{S_{e}, 1\right\}
\end{aligned}
$$

for $e \in[1, b]$,

$$
\begin{aligned}
& a_{0}^{-1}\left(a_{j_{1}-1} a_{j_{1}}^{-1}\right)\left(a_{j_{2}-1} a_{j_{2}}^{-1}\right) \ldots\left(a_{j_{e-1}-1} a_{j_{e-1}}^{-1}\right)\left(a_{j_{e}} a_{j_{e}-1}^{-1}\right) \\
& \left(a_{j_{e-1}} a_{j_{e-1}-1}^{-1}\right) \ldots\left(a_{j_{2}} a_{j_{2}-1}^{-1}\right)\left(a_{j_{1}} a_{j_{1}-1}^{-1}\right) \in\left\{\tilde{S}_{e}, 1\right\}
\end{aligned}
$$

for $e \in[1, \tilde{b}]$,

$$
\begin{gathered}
\omega=a_{\tilde{r}}\left(a_{\tilde{r}+i_{1}-1}^{-1} a_{\tilde{r}+i_{1}}\right)\left(a_{\tilde{r}+i_{2}-1}^{-1} a_{\tilde{r}+i_{2}}\right) \ldots\left(a_{\tilde{r}+i_{b}-1}^{-1} a_{\tilde{r}+i_{b}}\right) a_{\rho}^{-1}, \\
\tilde{\omega}=a_{0}^{-1}\left(a_{j_{1}-1} a_{j_{1}}^{-1}\right)\left(a_{j_{2}-1} a_{j_{2}}^{-1}\right) \ldots\left(a_{j_{\tilde{b}-1}} a_{j_{\tilde{b}}}^{-1}\right) a_{\tilde{r}} .
\end{gathered}
$$




\section{Setting}

(a) $\quad \hat{a}_{e}=a_{j_{e}} a_{j_{e}-1}^{-1}$ for $e \in[1, \tilde{b}], \quad \hat{a}_{\tilde{b}+e}=a_{\tilde{r}} a_{\tilde{r}+i_{e}}^{-1} a_{\tilde{r}+i_{e}-1} a_{\tilde{r}}^{-1}$ for $e \in[1, b]$ we see that

$$
\begin{gathered}
\hat{a}_{\tilde{b}+1}^{-1} \hat{a}_{\tilde{b}+2}^{-1} \ldots \hat{a}_{\tilde{b}+e-1}^{-1} \hat{a}_{\tilde{b}+e} \hat{a}_{\tilde{b}+e-1} \ldots \hat{a}_{\tilde{b}+2} \hat{a}_{\tilde{b}+1} \in\left\{S_{e}, 1\right\} \text { for } e \in[1, b] \\
a_{0}^{-1} \hat{a}_{1}^{-1} \hat{a}_{2}^{-1} \ldots \hat{a}_{e-1}^{-1} \hat{a}_{e} \hat{a}_{e-1} \ldots \hat{a}_{2} \hat{a}_{1} a_{0} \in\left\{\tilde{S}_{e}, 1\right\} \text { for } e \in[1, \tilde{b}] \\
\omega=\hat{a}_{\tilde{b}+1}^{-1} \hat{a}_{\tilde{b}+2}^{-1} \ldots \hat{a}_{\tilde{b}+b}^{-1} a_{\tilde{r}} a_{\rho}^{-1} \\
\tilde{\omega}=a_{0}^{-1} \hat{a}_{1}^{-1} \hat{a}_{2}^{-1} \ldots \hat{a}_{\tilde{b}}^{-1} a_{\tilde{r}}
\end{gathered}
$$

Since $S_{e}=S_{e}^{-1} \in W_{\mathcal{L}}, \tilde{S}_{e}=\tilde{S}_{e}^{-1} \in W_{\mathcal{L}}$, it follows by induction on $e$ that

$$
\begin{gathered}
\hat{a}_{\tilde{b}+e}=\hat{a}_{\tilde{b}+e}^{-1} \in W_{\mathcal{L}} \text { for } e \in[1, b], \\
a_{0}^{-1} \hat{a}_{e} a_{0}=a_{0}^{-1} \hat{a}_{e}^{-1} a_{0} \in W_{\mathcal{L}} \text { for } e \in[1, \tilde{b}] .
\end{gathered}
$$

Since $a_{0}$ normalizes $W_{\mathcal{L}}$ it follows that

$$
\hat{a}_{e}=\hat{a}_{e}^{-1} \in W_{\mathcal{L}} \text { for } e \in[1, \tilde{b}] .
$$

Since $a_{0}^{*} \mathcal{L} \cong \mathcal{L}$ we can write uniquely

$$
a_{0}=A_{0} f \text { with } A_{0} \in W_{\mathcal{L}}, f \in W^{\prime 0} .
$$

We set

$$
\begin{gathered}
A_{e}=\hat{a}_{e} \ldots \hat{a}_{2} \hat{a}_{1} a_{0} f^{-1} \quad(e \in[1, \tilde{b}]), \\
A_{\tilde{b}+e}=\hat{a}_{\tilde{b}+1} \hat{a}_{\tilde{b}+2} \ldots \hat{a}_{\tilde{b}+e} \hat{a}_{\tilde{b}} \ldots \hat{a}_{2} \hat{a}_{1} a_{0} f^{-1} \quad(e \in[1, b]) .
\end{gathered}
$$

From (f),(g),(h) we see that $A_{e} \in W_{\mathcal{L}}$ for $e \in[0, b+\tilde{b}]$. From the definitions we have

$$
\begin{array}{cc}
A_{e-1}^{-1} A_{e}=f S_{e} f^{-1} & (e \in[1, \tilde{b}]), \\
A_{\tilde{b}+e-1} A_{\tilde{b}+e}^{-1}=S_{e} & (e \in[1, b]) .
\end{array}
$$

We have

$$
\begin{aligned}
A_{\tilde{b}+b} & =\hat{a}_{\tilde{b}+1} \hat{a}_{\tilde{b}+2} \ldots \hat{a}_{\tilde{b}+b} \hat{a}_{\tilde{b}} \ldots \hat{a}_{2} \hat{a}_{1} a_{0} f^{-1}=\omega a_{\rho} \tilde{\omega}^{-1} f^{-1} \\
& =(\omega c)\left(c^{-1} a_{\rho} c\right)(\tilde{\omega} c)^{-1} f^{-1}=(\omega c) a_{0}(\tilde{\omega} c)^{-1} f^{-1}=(\omega c) A_{0} f(\tilde{\omega} c)^{-1} f^{-1} .
\end{aligned}
$$

In particular, $(\omega c) w f(\tilde{\omega} c)^{-1} f^{-1}=w^{\prime}$ for some $w \in W_{\mathcal{L}}$. We have $(\omega c) w=w_{1}(\omega c)$ for some $w_{1} \in W_{\mathcal{L}}$ hence $(\omega c) f(\tilde{\omega} c)^{-1} f^{-1}=w_{1}^{-1} w^{\prime}$ belongs to $W_{\mathcal{L}} \cap W^{\prime 0}{ }_{\mathcal{L}}=\{1\}$. Thus $(\omega c) f(\tilde{\omega} c)^{-1} f^{-1}=1$; that is, $f \in \mathfrak{F}$. We also see that

$$
A_{\tilde{b}+b}=(\omega c) A_{0} f(\tilde{\omega} c)^{-1} f^{-1}=(\omega c) A_{0}(\omega c)^{-1} .
$$

Thus to each $\left(a_{0}, a_{1}, \ldots, a_{\rho}\right) \in \mathcal{A}(W, c, \mathcal{L}, \mathbf{s}, \tilde{\mathbf{s}})$ we have associated $f \in \mathfrak{F}$ and an element $\left(A_{0}, A_{1}, \ldots, A_{b+\tilde{b}}\right)$ in $\mathcal{A}\left(W_{\mathcal{L}}, \omega c, \overline{\mathbf{Q}}_{l}, \mathbf{S},{ }^{f} \tilde{\mathbf{S}}\right)$. This defines the map $\Psi$ in the proposition.

Conversely, assume that we are given $f \in \mathfrak{F}$ and an element $\left(A_{0}, A_{1}, \ldots, A_{b+\tilde{b}}\right)$ in $\mathcal{A}\left(W_{\mathcal{L}}, \omega c, \overline{\mathbf{Q}}_{l}, \mathbf{S},{ }^{f} \tilde{\mathbf{S}}\right)$. We will construct a sequence $\left(a_{0}, a_{1}, \ldots, a_{\rho}\right)$ in $W$ as follows.

We set $a_{0}=A_{0} f$. We define $\hat{a}_{1}, \hat{a}_{2}, \ldots, \hat{a}_{\tilde{b}}$ inductively so that (i) holds. We define $\hat{a}_{\tilde{b}+1}, \hat{a}_{\tilde{b}+2}, \ldots, \hat{a}_{\tilde{b}+b}$ inductively so that $(\mathrm{j})$ holds. We define the elements $a_{0}, a_{1}, a_{2}, \ldots, a_{\tilde{r}}$ by induction as follows. Note that $a_{0}$ is already defined. Assume that $a_{0}, a_{1}, \ldots, a_{u-1}$ are already defined for some $u \in[1, \tilde{r}]$. If $u=j_{e}$ for some $e \in[1, \tilde{b}]$, we set $a_{u}=\hat{a}_{e} a_{u-1}$. If $u \notin \mathcal{I}_{\tilde{\mathbf{s}}}$, we set $a_{u}=a_{u-1} \tilde{s}_{u}$. This completes the 
definition of $a_{0}, a_{1}, a_{2}, \ldots, a_{\tilde{r}}$. We define the elements $a_{\tilde{r}}, a_{\tilde{r}+1}, \ldots, a_{\tilde{r}+r}$ by induction as follows. Note that $a_{\tilde{r}}$ is already defined. Assume that $a_{\tilde{r}}, a_{\tilde{r}+1}, \ldots, a_{\tilde{r}+u-1}$ are already defined for some $u \in[1, r]$. If $u=i_{e}$ for some $e \in[1, b]$ we set $a_{\tilde{r}+u}=a_{\tilde{r}+u-1} a_{\tilde{r}}^{-1} \hat{a}_{\tilde{b}+e} a_{\tilde{r}}$. If $u \notin \mathcal{I}_{\mathbf{s}}$, we set $a_{\tilde{r}+u}=s_{u} a_{\tilde{r}+u-1}$. This completes the definition of $a_{\tilde{r}}, a_{\tilde{r}+1}, \ldots, a_{\tilde{r}+r}$.

From the definitions we see that $\left(a_{0}, a_{1}, a_{2}, \ldots, a_{\rho}\right) \in \mathcal{A}(W, c, \mathcal{L}, \mathbf{s}, \tilde{\mathbf{s}})$. We have thus constructed a map

$$
\sqcup_{f \in \mathfrak{F}} \mathcal{A}\left(W_{\mathcal{L}}, \omega c, \overline{\mathbf{Q}}_{l}, \mathbf{S},{ }^{f} \tilde{\mathbf{S}}\right) \rightarrow \mathcal{A}(W, c, \mathcal{L}, \mathbf{s}, \tilde{\mathbf{s}}) .
$$

From the definitions we see that this is an inverse to $\Psi$. The proposition is proved.

\section{A BASIS FOR A SPACE OF INTERTWINING OPERATORS}

6.1. Let $\mathcal{L}, \tilde{\mathcal{L}} \in \mathcal{S}(T)$. Let $\mathbf{s}=\left(s_{1}, s_{2}, \ldots, s_{r}\right), \tilde{\mathbf{s}}=\left(\tilde{s}_{1}, \tilde{s}_{2}, \ldots, \tilde{s}_{\tilde{r}}\right)$ be two sequences in $\mathbf{I}$ such that $([\mathbf{s}] F)^{*} \mathcal{L} \cong \mathcal{L},([\tilde{\mathbf{s}}] F)^{*} \tilde{\mathcal{L}} \cong \tilde{\mathcal{L}}$. Define $\mathcal{I}_{\mathbf{s}} \subset[1, r]$ in terms of $\mathbf{s}, \mathcal{L}$ as in 2.4 or 5.1 ; define $\mathcal{I}_{\tilde{\mathbf{s}}} \subset[1, \tilde{r}]$ similarly in terms of $\tilde{\mathbf{s}}, \tilde{\mathcal{L}}$.

We set $\rho=r+\tilde{r}$. Until the end of 6.13 we fix a sequence $\mathbf{a}=\left(a_{0}, a_{1}, \ldots, a_{\rho}\right)$ in $W$ such that

$$
\begin{aligned}
& a_{j-1}^{-1} a_{j} \in\left\{1, \tilde{s}_{j}\right\} \text { for } j \in[1, \tilde{r}] \\
& a_{\tilde{r}+i} a_{\tilde{r}+i-1}^{-1} \in\left\{1, s_{i}\right\} \text { for } i \in[1, r] \\
& \text { if } j \in[1, \tilde{r}]-\mathcal{I}_{\tilde{\mathbf{s}}}, a_{j-1} \tilde{s}_{j}>a_{j-1} \text {, then } a_{j-1}^{-1} a_{j}=\tilde{s}_{j} ; \\
& \text { if } i \in[1, r]-\mathcal{I}_{\mathbf{s}}, s_{i} a_{\tilde{r}+i-1}>a_{\tilde{r}+i-1}, \text { then } a_{\tilde{r}+i} a_{\tilde{r}+i-1}^{-1}=s_{i} ; \\
& a_{\rho}=\mathbf{c}\left(a_{0}\right) .
\end{aligned}
$$

We define representatives $\ddot{a}_{i}$ for $a_{i}$ in $N(T)(i \in[0, \rho])$ as follows. We set $\ddot{a}_{0}=\dot{a}_{0}$. For $j \in[1, \tilde{r}]$ we define $\ddot{a}_{j}$ inductively by

$$
\begin{aligned}
& \ddot{a}_{j}=\ddot{a}_{j-1} \text { if } a_{j}=a_{j-1}, \\
& \ddot{a}_{j}=\ddot{a}_{j-1} \dot{\tilde{s}}_{j} \text { if } a_{j}=a_{j-1} \tilde{s}_{j} .
\end{aligned}
$$

For $i \in[1, r]$ we define $\ddot{a}_{\tilde{r}+i}$ inductively by

$\ddot{a}_{\tilde{r}+i}=\ddot{a}_{\tilde{r}+i-1}$ if $a_{\tilde{r}+i-1}=a_{\tilde{r}+i}$,

$\ddot{a}_{\tilde{r}+i}=\dot{s}_{i}^{-1} \ddot{a}_{\tilde{r}+i-1}$ if $a_{\tilde{r}+i}=s_{i} a_{\tilde{r}+i-1}$.

Consider the commutative diagrams

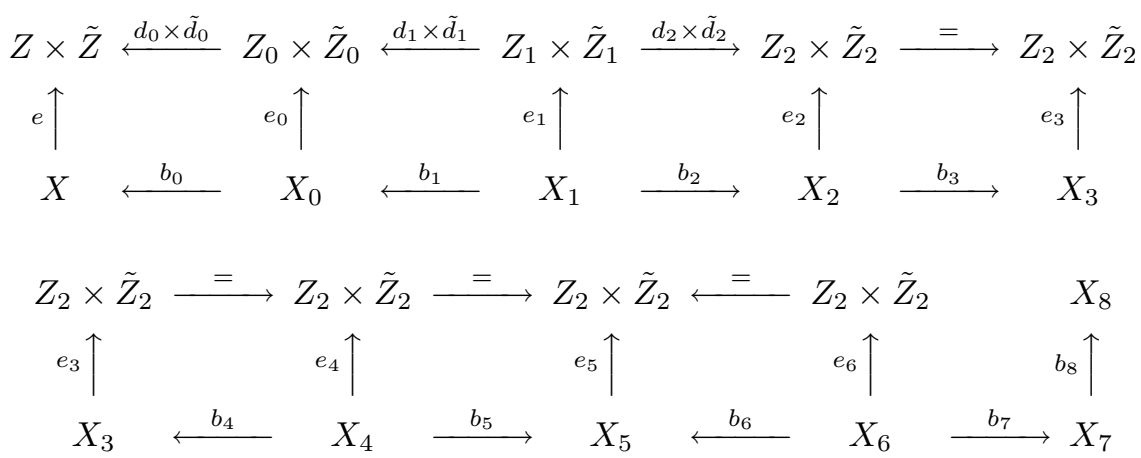

where the following notation is used.

$Z, Z_{i}, d_{i}$ are as in $2.6, \tilde{Z}, \tilde{Z}_{i}, \tilde{d}_{i}$ are the analogous objects defined in terms of $\tilde{\mathbf{s}}, \tilde{\mathcal{L}}$ instead of $\mathbf{s}, \mathcal{L}$.

$X$ is the set of all $\left(\left(B_{0}, B_{1}, \ldots, B_{r}\right),\left(\tilde{B}_{0}, \tilde{B}_{1}, \ldots, \tilde{B}_{\tilde{r}}\right)\right) \in \mathcal{B}^{[0, r]} \times \mathcal{B}^{[0, \tilde{r}]}$ such that $(A 0)-(A 5)$ below hold:

$(A 0) \operatorname{pos}\left(B_{0}, \tilde{B}_{j}\right)=a_{j}(j \in[0, \tilde{r}]), \operatorname{pos}\left(B_{i}, \tilde{B}_{\tilde{r}}\right)=a_{\tilde{r}+i}(i \in[0, r])$, 
$(A 1) \operatorname{pos}\left(B_{i-1}, B_{i}\right)=s_{i}$ if $i \in[1, r]-\mathcal{I}_{\mathbf{s}}$

$(A 2) \operatorname{pos}\left(B_{i-1}, B_{i}\right) \in\left\{1, s_{i}\right\}$ if $i \in \mathcal{I}_{\mathbf{s}}$,

$(A 3) \operatorname{pos}\left(\tilde{B}_{j-1}, \tilde{B}_{j}\right)=\tilde{s}_{j}$ if $j \in[1, \tilde{r}]-\mathcal{I}_{\tilde{\mathbf{s}}}$,

$(A 4) \operatorname{pos}\left(\tilde{B}_{j-1}, \tilde{B}_{j}\right) \in\left\{1, \tilde{s}_{j}\right\}$ if $j \in \mathcal{I}_{\tilde{\mathbf{s}}}$,

(A5) $F\left(B_{0}\right)=B_{r}, F\left(\tilde{B}_{0}\right)=\tilde{B}_{\tilde{r}}$.

$X_{0}$ is the set of all

$$
\left(\left(g_{0} U, g_{1} U, \ldots, g_{r} U\right),\left(\tilde{g}_{0} U, \tilde{g}_{1} U, \ldots, \tilde{g}_{\tilde{r}} U\right)\right) \in(G / U)^{[0, r]} \times(G / U)^{[0, \tilde{r}]}
$$

such that $(B 0)-(B 5)$ below hold:

$(B 0) k\left(g_{0}^{-1} \tilde{g}_{j}\right)=\ddot{a}_{j}(j \in[0, \tilde{r}]), k\left(g_{i}^{-1} \tilde{g}_{\tilde{r}}\right)=\ddot{a}_{\tilde{r}+i}(i \in[0, r])$,

(B1) $g_{i-1}^{-1} g_{i} \in P_{s_{i}}-B$ if $i \in[1, r]-\mathcal{I}_{\mathbf{s}}$,

(B2) $g_{i-1}^{-1} g_{i} \in P_{s_{i}}$ if $i \in \mathcal{I}_{\mathbf{s}}$,

(B3) $\tilde{g}_{j-1}^{-1} \tilde{g}_{j} \in P_{\tilde{s}_{j}}-B$ if $j \in[1, \tilde{r}]-\mathcal{I}_{\tilde{\mathbf{s}}}$,

(B4) $\tilde{g}_{j-1}^{-1} \tilde{g}_{j} \in P_{\tilde{s}_{j}}$ if $j \in \mathcal{I}_{\tilde{\mathbf{s}}}$,

(B5) $g_{r}^{-1} F\left(g_{0}\right) \in U, \tilde{g}_{\tilde{r}}^{-1} F\left(\tilde{g}_{0}\right) \in U$.

$b_{0}$ is

$$
\begin{aligned}
& \left(\left(g_{0} U, g_{1} U, \ldots, g_{r} U\right),\left(\tilde{g}_{0} U, \tilde{g}_{1} U, \ldots, \tilde{g}_{\tilde{r}} U\right)\right) \mapsto \\
& \left(\left(g_{0} B g_{0}^{-1}, g_{1} B g_{1}^{-1}, \ldots, g_{r} B g_{r}^{-1}\right),\left(\tilde{g}_{0} B \tilde{g}_{0}^{-1}, \tilde{g}_{1} B \tilde{g}_{1}^{-1}, \ldots, \tilde{g}_{\tilde{r}} B \tilde{g}_{\tilde{r}}^{-1}\right)\right) .
\end{aligned}
$$

$X_{1}$ is the set of all $\left(\left(g_{0}, g_{1}, \ldots, g_{r}\right),\left(\tilde{g}_{0}, \tilde{g}_{1}, \ldots, \tilde{g}_{\tilde{r}}\right)\right) \in G^{[0, r]} \times G^{[0, \tilde{r}]}$ such that $(B 0)-$ (B5) hold.

$b_{1}$ is

$\left.\left(\left(g_{0}, g_{1}, \ldots, g_{r}\right),\left(\tilde{g}_{0}, \tilde{g}_{1}, \ldots, \tilde{g}_{\tilde{r}}\right)\right) \mapsto\left(\left(g_{0} U, g_{1} U, \ldots, g_{r} U\right)\right),\left(\tilde{g}_{0} U, \tilde{g}_{1} U, \ldots, \tilde{g}_{\tilde{r}} U\right)\right)$.

$X_{2}$ is the set of all $\left(x, x^{\prime}, u, u^{\prime}, y_{0}, y_{1}, \ldots, y_{\rho}\right) \in G \times G \times U \times U \times G^{[0, \rho]}$ such that $(C 0)-\left(C^{\prime} 5\right)$ below hold:

(C0) $k\left(y_{z}\right)=\ddot{a}_{z}(z \in[0, \rho])$,

(C1) $y_{\tilde{r}+i-1} y_{\tilde{r}+i}^{-1} \in P_{s_{i}}-B$ if $i \in[1, r]-\mathcal{I}_{\mathbf{s}}$,

(C2) $y_{\tilde{r}+i-1} y_{\tilde{r}+i}^{-1} \in P_{s_{i}}$ if $i \in \mathcal{I}_{\mathbf{s}}$,

(C3) $y_{j-1}^{-1} y_{j} \in P_{\tilde{s}_{j}}-B$ if $j \in[1, \tilde{r}]-\mathcal{I}_{\tilde{\mathbf{s}}}$,

(C4) $y_{j-1}^{-1} y_{j} \in P_{\tilde{s}_{j}}$ if $j \in \mathcal{I}_{\tilde{\mathbf{s}}}$,

(C5) $u F\left(y_{0}\right)=y_{\rho} u^{\prime}$,

$\left(C^{\prime} 5\right) y_{0} x^{\prime}=x F\left(y_{0}\right), y_{\rho} y_{\tilde{r}}^{-1} x=u$.

$b_{2}$ is $\left(\left(g_{0}, g_{1}, \ldots, g_{r}\right),\left(\tilde{g}_{0}, \tilde{g}_{1}, \ldots, \tilde{g}_{\tilde{r}}\right)\right) \mapsto\left(x, x^{\prime}, u, u^{\prime}, y_{0}, y_{1}, \ldots, y_{\rho}\right)$ where

$$
\begin{aligned}
& x=g_{0}^{-1} F\left(g_{0}\right), x^{\prime}=\tilde{g}_{0}^{-1} F\left(\tilde{g}_{0}\right), u=g_{r}^{-1} F\left(g_{0}\right), u^{\prime}=\tilde{g}_{\tilde{r}}^{-1} F\left(\tilde{g}_{0}\right), \\
& y_{j}=g_{0}^{-1} \tilde{g}_{j}(j \in[0, \tilde{r}]), y_{\tilde{r}+i}=g_{i}^{-1} \tilde{g}_{\tilde{r}}(i \in[0, r]) .
\end{aligned}
$$

$X_{3}$ is the set of all $\left(u, u^{\prime}, y_{0}, y_{1}, \ldots, y_{\rho}\right) \in U^{2} \times G^{[0, \rho]}$ such that $(C 0)-(C 5)$ hold.

$b_{3}$ is $\left(x, x^{\prime}, u, u^{\prime}, y_{0}, y_{1}, \ldots, y_{\rho}\right) \mapsto\left(u, u^{\prime}, y_{0}, y_{1}, \ldots, y_{\rho}\right)$.

$X_{4}$ is the set of all $\left(u, u^{\prime}, v, v^{\prime}, \tilde{v}, \tilde{v}^{\prime}, y_{0}, y_{1}, \ldots, y_{\rho}\right) \in U^{6} \times G^{[0, \rho]}$ such that $(C 0)-$ (C4) hold and

$$
y_{0}=v \ddot{a}_{0} v^{\prime}, y_{\rho}=\tilde{v} F\left(\ddot{a}_{0}\right) \tilde{v}^{\prime}, u F(v) F\left(\ddot{a}_{0}\right) F\left(v^{\prime}\right)=\tilde{v} F\left(\ddot{a}_{0}\right) \tilde{v}^{\prime} u^{\prime} .
$$

$b_{4}$ is $\left(u, u^{\prime}, v, v^{\prime}, \tilde{v}, \tilde{v}^{\prime}, y_{0}, y_{1}, \ldots, y_{\rho}\right) \mapsto\left(u, u^{\prime}, y_{0}, y_{1}, \ldots, y_{\rho}\right)$.

$X_{5}$ is the set of all $\left(v, v^{\prime}, y_{0}, y_{1}, \ldots, y_{\rho}\right) \in U^{2} \times G^{[0, \rho]}$ such that $(C 0)-(C 4)$ hold and $y_{0}=v \ddot{a}_{0} v^{\prime}$.

$b_{5}$ is $\left(u, u^{\prime}, v, v^{\prime}, \tilde{v}, \tilde{v}^{\prime}, y_{0}, y_{1}, \ldots, y_{\rho}\right) \mapsto\left(v, v^{\prime}, y_{0}, y_{1}, \ldots, y_{\rho}\right)$. 
$X_{6}$ is the set of all $\left(\left(g_{0}, g_{1}, \ldots, g_{r}\right),\left(\tilde{g}_{0}, \tilde{g}_{1}, \ldots, \tilde{g}_{\tilde{r}}\right)\right) \in G^{[0, r]} \times G^{[0, \tilde{r}]}$ such that $(B 0)-(B 4)$ hold and $g_{0} \in U, \tilde{g}_{0} \in \ddot{a}_{0} U$.

$b_{6}$ is $\left(\left(g_{0}, g_{1}, \ldots, g_{r}\right),\left(\tilde{g}_{0}, \tilde{g}_{1}, \ldots, \tilde{g}_{\tilde{r}}\right)\right) \mapsto\left(v, v^{\prime}, y_{0}, y_{1}, \ldots, y_{\rho}\right)$ where

$$
v=g_{0}^{-1}, v^{\prime}=\ddot{a}_{0}^{-1} \tilde{g}_{0}, y_{j}=g_{0}^{-1} \tilde{g}_{j}(j \in[0, \tilde{r}]), y_{\tilde{r}+i}=g_{i}^{-1} \tilde{g}_{\tilde{r}}(i \in[0, r]) .
$$

$X_{7}$ is the set of all

$$
\left(\left(g_{0} U, g_{1} U, \ldots, g_{r} U\right),\left(\tilde{g}_{0} U, \tilde{g}_{1} U, \ldots, \tilde{g}_{\tilde{r}} U\right)\right) \in(G / U)^{[0, r]} \times(G / U)^{[0, \tilde{r}]}
$$

such that $(B 0)-(B 4)$ hold and $g_{0} \in U, \tilde{g}_{0} \in \ddot{a}_{0} U$.

$b_{7}$ is given by the same formula as $b_{1}$.

$X_{8}$ is the set of all $\left(\left(B_{0}, B_{1}, \ldots, B_{r}\right),\left(\tilde{B}_{0}, \tilde{B}_{1}, \ldots, \tilde{B}_{\tilde{r}}\right)\right) \in \mathcal{B}^{[0, r]} \times \mathcal{B}^{[0, \tilde{r}]}$ such that $(A 0)-(A 4)$ hold and $B_{0}=B, \tilde{B}_{0}=\ddot{a}_{0} B \ddot{a}_{0}^{-1}$.

$b_{8}$ is given by the same formula as $b_{0}$.

$e, e_{0}$ are the obvious imbeddings.

$e_{1}$ is

$\left(\left(g_{0}, g_{1}, \ldots, g_{r}\right),\left(\tilde{g}_{0}, \tilde{g}_{1}, \tilde{g}_{\tilde{r}}\right)\right) \mapsto\left(\left(g_{0}, g_{0} g_{1}^{-1}, \ldots, g_{r-1}^{-1} g_{r}\right),\left(\tilde{g}_{0}, \tilde{g}_{0} \tilde{g}_{1}^{-1}, \ldots, \tilde{g}_{\tilde{r}-1}^{-1} \tilde{g}_{\tilde{r}}\right)\right)$.

$e_{2}$ is $\left(x, x^{\prime}, u, u^{\prime}, y_{0}, y_{1}, \ldots, y_{\rho}\right) \mapsto \lambda\left(y_{0}, y_{1}, \ldots, y_{\rho}\right)$ where

$\lambda\left(y_{0}, y_{1}, \ldots, y_{\rho}\right)=\left(\left(y_{\tilde{r}} y_{\tilde{r}+1}^{-1}, y_{\tilde{r}+1} y_{\tilde{r}+2}^{-1}, \ldots, y_{\tilde{r}+r-1} y_{\tilde{r}+r}^{-1}\right),\left(y_{0}^{-1} y_{1}, y_{1}^{-1} y_{2}, \ldots, y_{\tilde{r}-1}^{-1} y_{\tilde{r}}\right)\right)$.

$e_{3}$ is $\left(u, u^{\prime}, y_{0}, y_{1}, \ldots, y_{\rho}\right) \mapsto \lambda\left(y_{0}, y_{1}, \ldots, y_{\rho}\right)$.

$e_{4}$ is $\left(u, u^{\prime}, v, v^{\prime}, \tilde{v}, \tilde{v}^{\prime}, y_{0}, y_{1}, \ldots, y_{\rho}\right) \mapsto \lambda\left(y_{0}, y_{1}, \ldots, y_{\rho}\right)$.

$e_{5}$ is $\left(v, v^{\prime}, y_{0}, y_{1}, \ldots, y_{\rho}\right) \mapsto \lambda\left(y_{0}, y_{1}, \ldots, y_{\rho}\right)$.

$e_{6}$ is

$$
\begin{aligned}
& \left(\left(g_{0}, g_{1}, \ldots, g_{r}\right),\left(\tilde{g}_{0}, \tilde{g}_{1}, \ldots, \tilde{g}_{\tilde{r}}\right)\right) \mapsto \\
& \left(\left(g_{0}^{-1} g_{1}, g_{1}^{-1} g_{2}, \ldots, g_{r-1}^{-1} g_{r}\right),\left(\tilde{g}_{0}^{-1} \tilde{g}_{1}, \tilde{g}_{1}^{-1} \tilde{g}_{2}, \ldots, \tilde{g}_{r-1}^{-1} \tilde{g}_{r}\right)\right) .
\end{aligned}
$$

6.2. Let $\underline{B}, \overline{\mathcal{L}}, \overline{\mathcal{L}}_{i}$ be as in $2.4,2.11$. Define $\underline{\tilde{B}}, \overline{\tilde{\mathcal{L}}}, \overline{\tilde{\mathcal{L}}}_{i}$ similarly, in terms of $\tilde{\mathbf{s}}, \tilde{\mathcal{L}}$ instead of $\mathbf{s}, \mathcal{L}$. Note that $\Gamma, \underline{B}$ act on $Z$ and $Z_{i}(i \in[0,2])$ as in 2.7. Similarly, $\Gamma, \underline{\tilde{B}}$ act on $\tilde{Z}$ and $\tilde{Z}_{i}(i \in[0,2])$. These actions give rise to (commuting) actions of $\Gamma \times \bar{\Gamma}, \underline{B} \times \underline{\tilde{B}}$ on $Z \times \tilde{Z}$ and $Z_{i} \times \tilde{Z}_{i}(i \in[0,2])$ hence to actions of $\Gamma \times \Gamma \times \underline{B} \times \underline{\tilde{B}}$. By 2.11 , $\overline{\mathcal{L}}$ and $\overline{\mathcal{L}}_{i}$ are $\underline{B}$-equivariant local systems with natural $\Gamma$-equivariant structures. Similarly, $\overline{\tilde{\mathcal{L}}}$ and $\overline{\tilde{\mathcal{L}}}_{i}$ are $\underline{\tilde{B}}$-equivariant local systems with natural $\Gamma$-equivariant structures. Hence $\overline{\mathcal{L}} \otimes \overline{\tilde{\mathcal{L}}}$ and $\overline{\mathcal{L}}_{i} \otimes \overline{\tilde{\mathcal{L}}}_{i}$ are $\underline{B} \times \underline{\tilde{B}}$-equivariant local systems on $Z \times \tilde{Z}$ and $Z_{i} \times \tilde{Z}_{i}$ with natural $\Gamma \times \Gamma$-equivariant structures. These structures give rise to $\Gamma \times \Gamma \times \underline{B} \times \underline{\tilde{B}}$-equivariant structures on $\overline{\mathcal{L}} \otimes \overline{\tilde{\mathcal{L}}}$ and $\overline{\mathcal{L}}_{i} \otimes \overline{\tilde{\mathcal{L}}}_{i}$. From the definitions we have

$$
\begin{aligned}
& \left(d_{0} \times \tilde{d}_{0}\right)^{*}(\overline{\mathcal{L}} \otimes \overline{\tilde{\mathcal{L}}})=\overline{\mathcal{L}}_{0} \otimes \overline{\tilde{\mathcal{L}}}_{0},\left(d_{1} \times \tilde{d}_{1}\right)^{*}\left(\overline{\mathcal{L}}_{0} \otimes \overline{\tilde{\mathcal{L}}}_{0}\right)=\overline{\mathcal{L}}_{1} \otimes \overline{\tilde{\mathcal{L}}}_{1}, \\
& \left(d_{2} \times \tilde{d}_{2}\right)^{*}\left(\overline{\mathcal{L}}_{2} \otimes \overline{\tilde{\mathcal{L}}}_{2}\right)=\overline{\mathcal{L}}_{1} \otimes \overline{\tilde{\mathcal{L}}}_{1},
\end{aligned}
$$

compatibly with the $\Gamma \times \Gamma \times \underline{B} \times \underline{\tilde{B}}$-equivariant structures.

\subsection{Let}

$$
\begin{aligned}
& \mathcal{T}=\left\{\left(\left(t_{0}, t_{1}, \ldots, t_{r}\right),\left(\tilde{t}_{0}, \tilde{t}_{1}, \ldots, \tilde{t}_{\tilde{r}}\right)\right) \in T^{[0, r]} \times T^{[0, \tilde{r}]}\right. \\
& \quad t_{r}=F\left(t_{0}\right), \tilde{t}_{\tilde{r}}=F\left(\tilde{t}_{0}\right), \tilde{t}_{j}=a_{j}^{-1}\left(t_{0}\right) \text { for } j \in[0, \tilde{r}], t_{i}=a_{\tilde{r}+i}\left(\tilde{t}_{\tilde{r}}\right) \text { for } i \in[0, r] .
\end{aligned}
$$

This is a subgroup of $T^{[0, r]} \times T^{[0, \tilde{r}]}$ isomorphic to the finite subgroup

$$
\mathcal{T}_{0}=\left\{t_{0} \in T ; a_{\tilde{r}}^{-1}\left(t_{0}\right)=F\left(a_{0}^{-1}\left(t_{0}\right)\right)\right\}
$$


of $T$ under $\left(\left(t_{i}\right),\left(\tilde{t}_{j}\right)\right) \mapsto t_{0}$. Hence $\mathcal{T}$ is finite.

$\Gamma \times \mathcal{T}$ acts on $X$ by

$$
\begin{aligned}
& \left(g,\left(t_{i}\right),\left(\tilde{t}_{j}\right)\right):\left(\left(B_{0}, B_{1}, \ldots, B_{r}\right),\left(\tilde{B}_{0}, \tilde{B}_{1}, \ldots, \tilde{B}_{\tilde{r}}\right)\right) \mapsto \\
& \left(\left(g B_{0} g^{-1}, g B_{1} g^{-1}, \ldots, g B_{r} g^{-1}\right),\left(g \tilde{B}_{0} g^{-1}, g \tilde{B}_{1} g^{-1}, \ldots, g \tilde{B}_{\tilde{r}} g^{-1}\right)\right) ;
\end{aligned}
$$

on $X_{0}$ by

$$
\begin{aligned}
& \left(g,\left(t_{i}\right),\left(\tilde{t}_{j}\right)\right):\left(\left(g_{0} U, g_{1} U, \ldots, g_{r} U\right),\left(\tilde{g}_{0} U, \tilde{g}_{1} U, \ldots, \tilde{g}_{\tilde{r}} U\right)\right) \mapsto \\
& \left(\left(g g_{0} t_{0}^{-1} U, g g_{1} t_{1}^{-1} U, \ldots, g g_{r} t_{r}^{-1} U\right),\left(g \tilde{g}_{0} \tilde{t}_{0}^{-1} U, g \tilde{g}_{1} \tilde{t}_{1}^{-1} U, \ldots, g \tilde{g}_{\tilde{r}} \tilde{t}_{\tilde{r}}^{-1} U\right)\right) ;
\end{aligned}
$$

on $X_{1}$ by

$$
\begin{aligned}
& \left(g,\left(t_{i}\right),\left(\tilde{t}_{j}\right)\right):\left(\left(g_{0}, g_{1}, \ldots, g_{r}\right),\left(\tilde{g}_{0}, \tilde{g}_{1}, \ldots, \tilde{g}_{\tilde{r}}\right)\right) \mapsto \\
& \left(\left(g g_{0} t_{0}^{-1}, g g_{1} t_{1}^{-1}, \ldots, g g_{r} t_{r}^{-1}\right),\left(g \tilde{g}_{0} \tilde{t}_{0}^{-1}, g \tilde{g}_{1} \tilde{t}_{1}^{-1}, \ldots, g \tilde{g}_{\tilde{r}} \tilde{t}_{\tilde{r}}^{-1}\right)\right) ;
\end{aligned}
$$

on $X_{2}$ by

$$
\begin{aligned}
& \left(g,\left(t_{i}\right),\left(\tilde{t}_{j}\right)\right):\left(x, x^{\prime}, u, u^{\prime}, y_{0}, y_{1}, \ldots, y_{\rho}\right) \mapsto\left(t_{0} x F\left(t_{0}\right)^{-1}, \tilde{t}_{0} x^{\prime} F\left(\tilde{t}_{0}\right)^{-1}, t_{r} u t_{r}^{-1},\right. \\
& \left.\tilde{t}_{\tilde{r}} u^{\prime} \tilde{t}_{\tilde{r}}^{-1}, t_{0} y_{0} \tilde{t}_{0}^{-1}, t_{0} y_{1} \tilde{t}_{1}^{-1}, \ldots, t_{0} y_{\tilde{r}} \tilde{t}_{\tilde{r}}^{-1}, t_{1} y_{\tilde{r}+1} \tilde{t}_{\tilde{r}}^{-1}, \ldots, t_{r} y_{\rho} \tilde{t}_{\tilde{r}}^{-1}\right) ;
\end{aligned}
$$

on $X_{3}$ by

$$
\begin{aligned}
& \left(g,\left(t_{i}\right),\left(\tilde{t}_{j}\right)\right):\left(u, u^{\prime}, y_{0}, y_{1}, \ldots, y_{\rho}\right) \mapsto \\
& \left(t_{r} u t_{r}^{-1}, \tilde{t}_{\tilde{r}} u^{\prime} \tilde{t}_{\tilde{r}}^{-1}, t_{0} y_{0} \tilde{t}_{0}^{-1}, t_{0} y_{1} \tilde{t}_{1}^{-1}, \ldots, t_{0} y_{\tilde{r}} \tilde{t}_{\tilde{r}}^{-1}, t_{1} y_{\tilde{r}+1} \tilde{t}_{\tilde{r}}^{-1}, \ldots, t_{r} y_{\rho} \tilde{t}_{\tilde{r}}^{-1}\right) ;
\end{aligned}
$$

on $X_{4}$ by

$$
\begin{aligned}
& \left(g,\left(t_{i}\right),\left(\tilde{t}_{j}\right)\right):\left(u, u^{\prime}, v, v^{\prime}, \tilde{v}, \tilde{v}^{\prime}, y_{0}, y_{1}, \ldots, y_{\rho}\right) \mapsto\left(t_{r} u t_{r}^{-1}, \tilde{t}_{\tilde{r}} u^{\prime} \tilde{t}_{\tilde{r}}^{-1},\right. \\
& \left.\quad t_{0} v t_{0}^{-1}, \tilde{t}_{0} v^{\prime} \tilde{t}_{0}^{-1}, t_{0} y_{0} \tilde{t}_{0}^{-1}, t_{0} y_{1} \tilde{t}_{1}^{-1}, \ldots, t_{0} y_{\tilde{r}} \tilde{t}_{\tilde{r}}^{-1}, t_{1} y_{\tilde{r}+1} \tilde{t}_{\tilde{r}}^{-1}, \ldots, t_{r} y_{\rho} \tilde{t}_{\tilde{r}}^{-1}\right) ;
\end{aligned}
$$

on $X_{5}$ by

$$
\begin{aligned}
& \left(g,\left(t_{i}\right),\left(\tilde{t}_{j}\right)\right):\left(v, v^{\prime}, \tilde{v}, \tilde{v}^{\prime}, y_{0}, y_{1}, \ldots, y_{\rho}\right) \mapsto \\
& \left(t_{0} v t_{0}^{-1}, \tilde{t}_{0} v^{\prime} \tilde{t}_{0}^{-1}, t_{0} y_{0} \tilde{t}_{0}^{-1}, t_{0} y_{1} \tilde{t}_{1}^{-1}, \ldots, t_{0} y_{\tilde{r}} \tilde{t}_{\tilde{r}}^{-1}, t_{1} y_{\tilde{r}+1} \tilde{t}_{\tilde{r}}^{-1}, \ldots, t_{r} y_{\rho} \tilde{t}_{\tilde{r}}^{-1}\right) ;
\end{aligned}
$$

on $X_{6}$ by

$$
\begin{aligned}
& \left(g,\left(t_{i}\right),\left(\tilde{t}_{j}\right)\right):\left(\left(g_{0}, g_{1}, \ldots, g_{r}\right),\left(\tilde{g}_{0}, \tilde{g}_{1}, \ldots, \tilde{g}_{\tilde{r}}\right)\right) \mapsto \\
& \left(\left(t_{0} g_{0} t_{0}^{-1}, t_{0} g_{1} t_{1}^{-1}, \ldots, t_{0} g_{r} t_{r}^{-1}\right),\left(t_{0} \tilde{g}_{0} \tilde{t}_{0}^{-1}, t_{0} \tilde{g}_{1} \tilde{t}_{1}^{-1}, \ldots, t_{0} \tilde{g}_{\tilde{r}} \tilde{t}_{\tilde{r}}^{-1}\right)\right) ;
\end{aligned}
$$

on $X_{7}$ by

$$
\begin{aligned}
& \left(g,\left(t_{i}\right),\left(\tilde{t}_{j}\right)\right):\left(\left(g_{0} U, g_{1} U, \ldots, g_{r} U\right),\left(\tilde{g}_{0} U, \tilde{g}_{1} U, \ldots, \tilde{g}_{\tilde{r}} U\right)\right) \mapsto \\
& \left(\left(t_{0} g_{0} t_{0}^{-1} U, t_{0} g_{1} t_{1}^{-1} U, \ldots, t_{0} g_{r} t_{r}^{-1} U\right),\left(t_{0} \tilde{g}_{0} \tilde{t}_{0}^{-1} U, t_{0} \tilde{g}_{1} \tilde{t}_{1}^{-1} U, \ldots, t_{0} \tilde{g}_{\tilde{r}} \tilde{t}_{\tilde{r}}^{-1} U\right)\right) ;
\end{aligned}
$$

on $X_{8}$ by

$$
\begin{aligned}
& \left(g,\left(t_{i}\right),\left(\tilde{t}_{j}\right)\right):\left(\left(B_{0}, B_{1}, \ldots, B_{r}\right),\left(\tilde{B}_{0}, \tilde{B}_{1}, \ldots, \tilde{B}_{\tilde{r}}\right)\right) \mapsto \\
& \left(\left(t_{0} B_{0} t_{0}^{-1}, t_{0} B_{1} t_{0}^{-1}, \ldots, t_{0} B_{r} t_{0}^{-1}\right),\left(t_{0} \tilde{B}_{0} t_{0}^{-1}, t_{0} \tilde{B}_{1} t_{0}^{-1}, \ldots, t_{0} \tilde{B}_{\tilde{r}} t_{0}^{-1}\right)\right) .
\end{aligned}
$$

The maps $b_{i}$ are compatible with the $\Gamma \times \mathcal{T}$-actions. Let

$$
\begin{aligned}
& \mathcal{G}=\left\{\left(\left(b_{0}, b_{1}, \ldots, b_{r}\right),\left(\tilde{b}_{0}, \tilde{b}_{1}, \ldots, \tilde{b}_{\tilde{r}}\right)\right) \in \underline{B} \times \underline{\tilde{B}} ;\right. \\
& \left.\quad\left(\left(k\left(b_{0}\right), k\left(b_{1}\right), \ldots, k\left(b_{r}\right)\right),\left(k\left(\tilde{b}_{0}\right), k\left(\tilde{b}_{1}\right), \ldots, k\left(\tilde{b}_{\tilde{r}}\right)\right)\right) \in \mathcal{T}\right\},
\end{aligned}
$$


a subgroup of $\underline{B} \times \underline{\tilde{B}}$. The $\Gamma \times \mathcal{T}$-action on $X_{6}$ extends to a $\Gamma \times \mathcal{G}$-action on $X_{6}$ :

$\left(g,\left(b_{i}\right),\left(\tilde{b}_{j}\right)\right):\left(\left(g_{0}, g_{1}, \ldots, g_{r}\right),\left(\tilde{g}_{0}, \tilde{g}_{1}, \ldots, \tilde{g}_{\tilde{r}}\right)\right) \mapsto$

$\left(\left(k\left(b_{0}\right) g_{0} b_{0}^{-1}, k\left(b_{0}\right) g_{1} b_{1}^{-1}, \ldots, k\left(b_{0}\right) g_{r} b_{r}^{-1}\right),\left(k\left(b_{0}\right) \tilde{g}_{0} \tilde{b}_{0}^{-1}, k\left(b_{0}\right) \tilde{g}_{1} \tilde{b}_{1}^{-1}, \ldots, k\left(b_{0}\right) \tilde{g}_{\tilde{r}} \tilde{b}_{\tilde{r}}^{-1}\right)\right)$.

\subsection{Now}

(a) $b_{0}$ is a principal $\mathcal{T}$-bundle

$\left(\mathcal{T}\right.$ acts on $X_{0}$ by restriction of the $\Gamma \times \mathcal{T}$-action) and induces an isomorphism $\mathcal{T} \backslash X_{0} \stackrel{\sim}{\rightarrow} X$. (See [L4, 3.4].)

(b) $b_{1}$ is a principal $U^{[0, r]} \times U^{[0, \tilde{r}]}$-bundle.

(c) $b_{2}$ is a principal $\Gamma$-bundle

( $\Gamma$ acts on $X_{1}$ by restriction of the $\Gamma \times \mathcal{T}$-action) and induces an isomorphism $\Gamma \backslash X_{1} \stackrel{\sim}{\rightarrow} X_{2}$. (See [L4, 3.8].)

(d) $b_{3}$ is an isomorphism.

(e) $b_{4}$ is a quasi-vector bundle (see [L4, 3.2]) with fibres of $\operatorname{dimension} 2(\operatorname{dim} U-$ $\left.l\left(a_{0}\right)\right)$.

(f) $b_{5}$ is a quasi-vector bundle with fibres of dimension $2\left(\operatorname{dim} U-l\left(a_{0}\right)\right)$.

(g) $b_{6}$ is an isomorphism.

(h) $b_{7}$ is a principal $U^{[0, r]} \times U^{[0, \tilde{r}]}$-bundle.

(i) $b_{8}$ is an isomorphism. (See [L4, 3.24].)

6.5. Now $\mathcal{T}$ is naturally a subgroup of $\underline{B} \times \underline{\tilde{B}}$ and $\Gamma$ is a subgroup of $\Gamma \times \Gamma$ (the diagonal) hence $\Gamma \times \mathcal{T}$ is a subgroup of $\Gamma \times \Gamma \times \underline{B} \times \underline{\tilde{B}}$. Hence the actions in 6.2 give by restriction actions of $\Gamma \times \mathcal{T}$ on $Z \times \tilde{Z}$ and $Z_{i} \times \tilde{Z}_{i}(i \in[0,2])$ and the equivariant structures on $\overline{\mathcal{L}} \otimes \overline{\tilde{\mathcal{L}}}$ and $\overline{\mathcal{L}}_{i} \otimes \overline{\tilde{\mathcal{L}}}_{i}$ in 6.2 restrict to $\Gamma \times \mathcal{T}$-equivariant structures on these local systems. Since $e$ and $e_{i}(i \in[0,6])$ are compatible with the $\Gamma \times \mathcal{T}$ actions, we see that the local systems $\mathcal{E}=e^{*}(\overline{\mathcal{L}} \nabla \overline{\tilde{\mathcal{L}}}), \mathcal{E}_{i}=e_{i}^{*}\left(\overline{\mathcal{L}}_{i} \otimes \overline{\tilde{\mathcal{L}}}_{i}\right),(i \in[0,2])$ and $\mathcal{E}_{i}=e_{i}^{*}\left(\overline{\mathcal{L}}_{2} \otimes \overline{\tilde{\mathcal{L}}}_{2}\right),(i \in[3,6])$ have natural $\Gamma \times \mathcal{T}$-equivariant structures. Moreover, the $\Gamma \times \mathcal{T}$-equivariant structure on $\mathcal{E}_{6}$ extends to a $\Gamma \times \mathcal{G}$-equivariant structure since $e_{6}$ is compatible with the $\Gamma \times \mathcal{G}$ actions (see 6.3). Since the restriction of the $\Gamma \times \mathcal{G}$ action on $X_{6}$ to the subgroup $U^{[0, r]} \times U^{[0, \tilde{r}]}$ is the free action which makes $X_{6}$ a principal bundle over $X_{7}$ (see 6.4(h)), it follows that there is a well defined local system $\mathcal{E}_{7}$ on $X_{7}$ with a natural $\Gamma \times \mathcal{T}$-equivariant structure such that $b_{7}^{*} \mathcal{E}_{7}=\mathcal{E}_{6}$. Since $b_{8}$ is an isomorphism, there is a well defined local system $\mathcal{E}_{8}$ on $X_{7}$ with a natural $\Gamma \times \mathcal{T}$-equivariant structure such that $b_{8}^{*} \mathcal{E}_{8}=\mathcal{E}_{7}$. We have

(a) $\quad b_{6}^{*} \mathcal{E}_{5}=\mathcal{E}_{6} ; \quad b_{7}^{*} \mathcal{E}_{7}=\mathcal{E}_{6} ; \quad b_{8}^{*} \mathcal{E}_{8}=\mathcal{E}_{7} ;$

compatibly with the $\Gamma \times \mathcal{T}$-equivariant structures. We show:

(b) $\mathcal{T}$ acts trivially on any stalk of $\mathcal{E}$.

(c) $\Gamma$ acts trivially on any stalk of $\mathcal{E}_{i}$ (if $i \in[2,8]$ ).

(b) follows from the fact that $\underline{B} \times \underline{\underline{B}}$ acts trivially on $Z \times \tilde{Z}$ and, being connected, it acts trivially on any stalk of $\overline{\mathcal{L}} \otimes \overline{\tilde{\mathcal{L}}}$. Now (c) follows from the fact that $\Gamma \times \Gamma$ acts trivially on $Z_{2} \times \tilde{Z}_{2}$ and on any stalk of $\overline{\mathcal{L}}_{2} \otimes \overline{\tilde{\mathcal{L}}}_{2}$.

6.6. For any $h \in[0, \tilde{r}]$ let $\mathfrak{Y}_{h}$ be the set of all $\left(\tilde{B}_{0}, \tilde{B}_{1}, \ldots, \tilde{B}_{h}\right) \in \mathcal{B}^{[0, h]}$ such that $\operatorname{pos}\left(B, \tilde{B}_{j}\right)=a_{j}(j \in[0, h])$, $\operatorname{pos}\left(\tilde{B}_{j-1}, \tilde{B}_{j}\right)=\tilde{s}_{j}$ if $j \in[1, h], j \notin \mathcal{I}_{\tilde{\mathbf{s}}}$, 
$\operatorname{pos}\left(\tilde{B}_{j-1}, \tilde{B}_{j}\right) \in\left\{1, \tilde{s}_{j}\right\}$ if $j \in[1, h] \cap \mathcal{I}_{\tilde{\mathbf{s}}}$,

$\tilde{B}_{0}=\ddot{a}_{0} B \ddot{a}_{0}^{-1}$.

Note that $\mathfrak{Y}_{0}$ is a point. Moreover, if $h \in[1, \tilde{r}]$, then we have an obvious map $\mathfrak{Y}_{h} \rightarrow \mathfrak{Y}_{h-1}$ which is

-an isomorphism if $a_{h}=a_{h-1} \tilde{s}_{h}<a_{h-1}$;

-an isomorphism if $h \in \mathcal{I}_{\tilde{\mathbf{s}}}, a_{h-1} \tilde{s}_{h}>a_{h-1}=a_{h}$;

-a line bundle minus the zero section if $h \notin \mathcal{I}_{\tilde{\mathbf{s}}}, a_{h-1} \tilde{s}_{h}<a_{h-1}=a_{h}$;

-a line bundle if $a_{h}=a_{h-1} \tilde{s}_{h}>a_{h-1}$;

-a line bundle if $h \in \mathcal{I}_{\tilde{\mathbf{s}}}, a_{h-1} \tilde{s}_{h}<a_{h-1}=a_{h}$.

For any $h \in[0, r]$ let $\mathfrak{Y}_{\tilde{r}+h}$ be the set of all

$$
\left(\left(B_{0}, B_{1}, \ldots, B_{h}\right),\left(\tilde{B}_{0}, \tilde{B}_{1}, \ldots, \tilde{B}_{\tilde{r}}\right)\right) \in \mathcal{B}^{[0, h]} \times \mathcal{B}^{[0, \tilde{r}]}
$$

such that

$$
\begin{aligned}
& \operatorname{pos}\left(B_{0}, \tilde{B}_{j}\right)=a_{j}(j \in[0, \tilde{r}]), \operatorname{pos}\left(B_{i}, \tilde{B}_{\tilde{r}}\right)=a_{\tilde{r}+i}(i \in[0, h]), \\
& \operatorname{pos}\left(B_{i-1}, B_{i}\right)=s_{i} \text { if } i \in[1, h], j \notin \mathcal{I}_{\mathbf{s}}, \\
& \operatorname{pos}\left(B_{i-1}, B_{i}\right) \in\left\{1, s_{i}\right\} \text { if } i \in[1, h] \cap \mathcal{I}_{\mathbf{s}}, \\
& \operatorname{pos}\left(\tilde{B}_{j-1}, \tilde{B}_{j}\right)=\tilde{s}_{j} \text { if } j \in[1, \tilde{r}], j \notin \mathcal{I}_{\tilde{\mathbf{s}}}, \\
& \operatorname{pos}\left(\tilde{B}_{j-1}, \tilde{B}_{j}\right) \in\left\{1, \tilde{s}_{j}\right\} \text { if } j \in \mathcal{I}_{\tilde{\mathbf{s}}}, \\
& B_{0}=B, \tilde{B}_{0}=\ddot{a}_{0} B \ddot{a}_{0}^{-1} .
\end{aligned}
$$

Note that $\mathfrak{Y}_{\tilde{r}}$ in the last definition may be identified with $\mathfrak{Y}_{\tilde{r}}$ in the earlier one. Moreover, if $h \in[1, r]$, then we have an obvious map $\mathfrak{Y}_{\tilde{r}+h} \rightarrow \mathfrak{Y}_{\tilde{r}+h-1}$ which is

-an isomorphism if $a_{\tilde{r}+h}=s_{h} a_{\tilde{r}+h-1}<a_{\tilde{r}+h-1}$;

-an isomorphism if $h \in \mathcal{I}_{\mathbf{s}}, s_{h} a_{\tilde{r}+h-1}>a_{\tilde{r}+h-1}=a_{\tilde{r}+h}$;

-a line bundle minus the zero section if $h \notin \mathcal{I}_{\mathbf{s}}, s_{h} a_{\tilde{r}+h-1}<a_{\tilde{r}+h-1}=a_{\tilde{r}+h}$;

-a line bundle if $a_{\tilde{r}+h}=s_{h} a_{\tilde{r}+h-1}>a_{\tilde{r}+h-1}$;

-a line bundle if $h \in \mathcal{I}_{\mathbf{s}}, s_{h} a_{\tilde{r}+h-1}<a_{\tilde{r}+h-1}=a_{\tilde{r}+h}$.

6.7. Assume that for some $h \in[1, r]-\mathcal{I}_{\mathbf{s}}$ we have $a:=a_{\tilde{r}+h}=a_{\tilde{r}+h-1}, s:=s_{h}$, $s a<a$. We show that

(a) $H_{c}^{*}\left(X_{8}, \mathcal{E}_{8}\right)=0$.

We have an obvious map $\phi: X_{8} \rightarrow \mathfrak{Y}_{\tilde{r}+h-1}$. (See 6.6.) It is enough to show that for any $p=\left(\left(B_{0}, \ldots, B_{h-1}\right),\left(\tilde{B}_{0}, \tilde{B}_{1}, \ldots, t B_{\tilde{r}}\right)\right) \in \mathfrak{Y}_{\tilde{r}+h-1}$ we have $H_{c}^{*}\left(\mathfrak{Y}^{\prime}, \mathcal{E}_{8}\right)=0$ where $\mathfrak{Y}^{\prime}=\phi^{-1}(p)$. We may identify $\mathfrak{Y}^{\prime}$ with the set of all $\left(B_{h}, B_{h+1}, \ldots, B_{r}\right) \in$ $\mathcal{B}^{[h, r]}$ such that

$$
\begin{aligned}
& \operatorname{pos}\left(B_{i}, \tilde{B}_{\tilde{r}}\right)=a_{\tilde{r}+i}(i \in[h, r]), \\
& \operatorname{pos}\left(B_{i-1}, B_{i}\right) \in\left\{s_{i}, 1\right\} \text { if } i \in[h+1, r] \cap \mathcal{I}_{\mathbf{s}} . \\
& \operatorname{pos}\left(B_{i-1}, B_{i}\right)=s_{i} \text { if } i \in[h, r], i \notin \mathcal{I}_{\mathbf{s}} .
\end{aligned}
$$

Since $s a<a$, there is a unique $D \in \mathcal{B}$ such that $\operatorname{pos}\left(B_{h-1}, D\right)=s, \operatorname{pos}\left(D, \tilde{B}_{\tilde{r}}\right)=$ sa. Pick $C \in \mathcal{B}$ such that $\operatorname{pos}\left(\tilde{B}_{\tilde{r}}, C\right)=a^{-1} w_{\mathbf{I}}$. Since $\operatorname{pos}(D, C)=s w_{\mathbf{I}}, V:=$ $U_{D} \cap U_{C}$ is a one-dimensional connected unipotent group. Now $\tilde{B}_{\tilde{r}}$ is the unique Borel such that $\operatorname{pos}\left(D, \tilde{B}_{\tilde{r}}\right)=s a, \operatorname{pos}\left(\tilde{B}_{\tilde{r}}, C\right)=a^{-1} w_{\mathbf{I}}$. Hence it is normalized by $V$ and $V \subset U_{\tilde{B}_{\tilde{r}}}$. Since $\operatorname{pos}\left(B_{h-1}, C\right)=w_{\mathbf{I}}$, we have $U_{B_{h-1}} \cap U_{C}=\{1\}$ hence $U_{B_{h-1}} \cap V=\{1\}$. Let

$$
\begin{aligned}
& \Xi=\left\{E \in \mathcal{B} ; \operatorname{pos}\left(B_{h-1}, E\right)=s, \operatorname{pos}\left(E, \tilde{B}_{\tilde{r}}\right)=a\right\} \\
& =\left\{E \in \mathcal{B} ; \operatorname{pos}\left(B_{h-1}, E\right)=s, \operatorname{pos}(E, D)=s\right\} .
\end{aligned}
$$

Since $V \subset U_{D}, V \cap U_{B_{h-1}}=\{1\}, V$ acts simply transitively (by conjugation) on the affine line $\left\{E^{\prime} \in \mathcal{B} ; \operatorname{pos}\left(E^{\prime}, D\right)=s\right\}=\Xi \sqcup\left\{B_{h-1}\right\}$. Pick $B_{h} \in \xi$. Define 
$v_{0} \in V-\{1\}$ by $v_{0} B_{h} v_{0}^{-1}=B_{h-1}$. Let

$$
\mathfrak{Y}^{\prime \prime}=\left\{\left(B_{h+1}, \ldots, B_{r}\right) \in \mathcal{B}^{[h+1, r]} ;\left(B_{h}, B_{h+1}, \ldots, B_{r}\right) \in \mathfrak{Y}^{\prime}\right\} .
$$

The map $\zeta:\left(V-\left\{v_{0}\right\}\right) \times \mathfrak{Y}^{\prime \prime} \rightarrow \mathfrak{Y}^{\prime}$,

$$
\left(v,\left(B_{h+1}, \ldots, B_{r}\right)\right) \mapsto\left(v B_{h} v^{-1}, v B_{h+1} v^{-1}, \ldots, v B_{r} v^{-1}\right)
$$

is an isomorphism. Hence it is enough to show that

$$
H_{c}^{*}\left(\left(V-\left\{v_{0}\right\}\right) \times \mathfrak{Y}^{\prime \prime}, \zeta^{*} \mathcal{E}_{8}\right)=0 .
$$

Let $\pi^{\prime \prime}:\left(V-\left\{v_{0}\right\}\right) \times \mathfrak{Y}^{\prime \prime} \rightarrow \mathfrak{Y}^{\prime \prime}$ be the projection. It is enough to show that for any $p^{\prime}=\left(B_{h+1}, \ldots, B_{r}\right) \in \mathfrak{Y}^{\prime \prime}$ we have $H_{c}^{*}\left(\pi^{\prime \prime-1}\left(p^{\prime}\right), \zeta^{*} \mathcal{E}_{8}\right)=0$ or equivalently that $H_{c}^{*}\left(V-\left\{v_{0}\right\}, \zeta^{\prime *} \mathcal{E}_{8}\right)=0$ where $\zeta^{\prime}: V-\left\{v_{0}\right\} \rightarrow X_{8}$ is

$$
v \mapsto\left(\left(B_{0}, B_{1}, \ldots, B_{h-1}, v B_{h} v^{-1}, v B_{h+1} v^{-1}, \ldots, v B_{r} v^{-1}\right),\left(\tilde{B}_{0}, \tilde{B}_{1}, \ldots, \tilde{B}_{\tilde{r}}\right)\right) .
$$

Since $\zeta^{* *} \mathcal{E}_{8}$ is a local system of rank 1 on $V-\left\{v_{0}\right\} \cong \mathbf{k}^{*}$ with monodromy of finite order invertible in $\mathbf{k}$, it is enough to show that $\zeta^{\prime *} \mathcal{E}_{8} \neq \overline{\mathbf{Q}}_{l}$. We can find $\epsilon=\left(\left(g_{0}, g_{1}, \ldots, g_{r}\right),\left(\tilde{g}_{0}, \tilde{g}_{1}, \ldots, \tilde{g}_{\tilde{r}}\right)\right) \in X_{6}$ such that

$$
b_{8}\left(b_{7}(\epsilon)\right)=\left(\left(B_{0}, B_{1}, \ldots, B_{h-1}, B_{h}, B_{h+1}, \ldots, B_{r}\right),\left(\tilde{B}_{0}, \tilde{B}_{1}, \ldots, \tilde{B}_{\tilde{r}}\right)\right),
$$

$g_{h-1}^{-1} \tilde{g}_{\tilde{r}}=\ddot{a}$ and $V=g_{h-1} y_{s_{h}}(\mathbf{k}) g_{h-1}^{-1}$. Define $\lambda_{0} \in \mathbf{k}$ by $y_{s_{h}}\left(\lambda_{0}\right) g_{h-1}^{-1} g_{h} \in B$. Define $\tilde{\zeta}: \mathbf{k}-\left\{\lambda_{0}\right\} \rightarrow X_{6}$ by

$$
\lambda \mapsto\left(\left(g_{0}, g_{1}, \ldots, g_{h-1}, g_{h-1} y_{s_{h}}(\lambda) g_{h-1}^{-1} g_{h}, \ldots, g_{h-1} y_{s_{h}}(\lambda) g_{h-1}^{-1} g_{r}\right),\left(\tilde{g}_{0}, \tilde{g}_{1}, \ldots, \tilde{g}_{\tilde{r}}\right)\right) .
$$

We have $\zeta^{\prime *} \mathcal{E}_{8}=\tilde{\zeta}^{*} \mathcal{E}_{6}$. It is enough to prove that $\tilde{\zeta}^{*} \mathcal{E}_{6} \neq \overline{\mathbf{Q}}_{l}$ or that $\tilde{\zeta}^{*} e_{6}^{*}\left(\overline{\mathcal{L}}_{2} \otimes \overline{\tilde{\mathcal{L}}}_{2}\right) \not$ $\overline{\mathbf{Q}}_{l}$. Note that $e_{6} \tilde{\zeta}: \mathbf{k}-\left\{\lambda_{0}\right\} \rightarrow Z_{2} \times \tilde{Z}_{2}$ is

$$
\lambda \mapsto\left(\left(y_{1}, y_{2}, y_{h-1}, y_{s_{h}}(\lambda) y_{h}, y_{h+1}, \ldots, y_{r}\right),\left(\tilde{y}_{1}, \tilde{y}_{2}, \ldots, \tilde{y}_{\tilde{r}}\right)\right)
$$

where

$$
\left(\left(y_{1}, y_{2}, y_{h-1}, y_{h}, y_{h+1}, \ldots, y_{r}\right),\left(\tilde{y}_{1}, \tilde{y}_{2}, \ldots, \tilde{y}_{\tilde{r}}\right)\right)=e_{6}(\epsilon) .
$$

From this and the definition of $\overline{\mathcal{L}}_{2}=\underline{\mathcal{L}}$ (see 2.4) we see that $\left(e_{6} \tilde{\zeta}\right)^{*}\left(\overline{\mathcal{L}}_{2} \otimes \overline{\tilde{\mathcal{L}}}_{2}\right)$ is isomorphic to the inverse image of $\mathcal{L}$ under a map $\mathbf{k}-\left\{\lambda_{0}\right\} \rightarrow T$ of the form

$$
\lambda \mapsto t^{\prime} \dot{s}_{1} \ldots \dot{s}_{h-1} k\left(y_{s_{h}}\left(\lambda-\lambda_{0}\right)\right) \dot{s}_{h}^{-1} \ldots \dot{s}_{1}^{-1}
$$

where $t^{\prime}$ is a fixed element of $T$. This is also of the form $\lambda \mapsto \beta\left(\lambda-\lambda_{0}\right) t^{\prime}$ where $\beta: \mathbf{k}^{*} \rightarrow T$ is one of the two coroots with associated reflection $s_{1} s_{2} \ldots s_{h} \ldots s_{2} s_{1}$. The desired result follows from the fact that $\beta^{*}(\mathcal{L}) \neq \overline{\mathbf{Q}}_{l}$. (Recall that $h \notin \mathcal{I}_{\mathbf{s}}$ ).

6.8. Assume that for some $h \in[1, \tilde{r}]-\mathcal{I}_{\tilde{\mathbf{s}}}$ we have $a:=a_{h}=a_{h-1}, s:=\tilde{s}_{h}$, as $<a$. We have

(a) $H_{c}^{*}\left(X_{8}, \mathcal{E}_{8}\right)=0$.

The proof is entirely similar to that of $6.7(\mathrm{a})$. 
6.9. Let

$N_{\mathbf{a}}=\left|\left\{h \in[1, \tilde{r}] ; a_{h-1} \leq a_{h} \geq a_{h-1} \tilde{s}_{h}\right\}\right|+\left|\left\{h \in[1, r] ; a_{\tilde{r}+h-1} \leq a_{\tilde{r}+h} \geq s_{h} a_{\tilde{r}+h-1}\right\}\right|$.

Consider the following condition on $\mathbf{a}$ :

(a) for any $h \in[1, r]-\mathcal{I}_{\mathbf{s}}$ we have $a_{\tilde{r}+h}=s_{h} a_{\tilde{r}+h-1}$; for any $h \in[1, \tilde{r}]-\mathcal{I}_{\tilde{\mathbf{s}}}$ we have $a_{h}=a_{h-1} \tilde{s}_{h}$.

If a satisfies (a), we have the following results:

(b) $X_{8}$ is isomorphic to an affine space of dimension $N_{\mathbf{a}}$.

(c) $X$ has pure dimension $N_{\mathbf{a}}$.

Indeed, from the results in 6.6 we see by induction on $h \in[0, \rho]$ that $\mathfrak{Y}_{h}$ is an affine space. (In this case each of the maps $\mathfrak{Y}_{h} \rightarrow \mathfrak{Y}_{h-1},(h \in[1, \rho])$ in 6.6 is either an isomorphism or an affine line bundle.) The same argument yields the dimension of each $\mathfrak{Y}_{h}$. This yields (b). Now (c) follows from (b) and the results in 6.4.

6.10. We assume that a satisfies 6.9 (a). Define $\left.x=\left(\left(g_{i}\right), \tilde{g}_{j}\right)\right) \in G^{[0, r]} \times G^{[0, \tilde{r}]}$ by $g_{i}=\ddot{a}_{\tilde{r}} \ddot{\tilde{r}}_{\tilde{r}+i}^{-1}, \tilde{g}_{j}=\ddot{a}_{j}$. Our assumption on a implies that $x \in X_{6}$. From the definitions we see that $x$ is a fixed point of the $\mathcal{T}$-action 6.3 on $X_{6}$. Hence, in the $\Gamma \times \mathcal{T}$-structure of $\mathcal{E}_{6}, \mathcal{T}$ acts on the stalk $\mathcal{E}_{6, x}$ of $\mathcal{E}_{6}$ at $x$. We show:

(a) the $\mathcal{T}$-action on $\mathcal{E}_{6, x}$ is trivial if and only if $\mathcal{L} \otimes\left(a_{0}^{-1}\right)^{*} \tilde{\mathcal{L}} \cong \overline{\mathbf{Q}}_{l}$.

Let

$$
\mathcal{J}=\left\{i \in[1, r] ; a_{\tilde{r}+i-1}=a_{\tilde{r}+i}\right\}, \tilde{\mathcal{J}}=\left\{j \in[1, \tilde{r}] ; a_{j-1}=a_{j}\right\}
$$

From our assumption on a we see that $\mathcal{J} \subset \mathcal{I}_{\mathbf{s}}, \tilde{\mathcal{J}} \subset \mathcal{I}_{\tilde{\mathbf{s}}}$. Let $f^{\mathcal{J}}: \mathcal{Y}^{\mathcal{J}} \rightarrow T$ be as in 2.4; let $\tilde{f}^{\tilde{\mathcal{J}}}: \tilde{\mathcal{Y}}^{\tilde{\mathcal{J}}} \rightarrow T$ be the analogous map defined in terms of $\tilde{\mathbf{s}}, \tilde{\mathcal{L}}, \tilde{\mathcal{J}}$ instead of $\mathbf{s}, \mathcal{L}, \mathcal{J}$. Define sequences $\mathbf{s}_{\mathcal{J}}=\left(s_{1}^{\prime}, s_{2}^{\prime}, \ldots, s_{r}^{\prime}\right), \tilde{\mathbf{s}}_{\tilde{\mathcal{J}}}=\left(\tilde{s}_{1}^{\prime}, \tilde{s}_{2}^{\prime}, \ldots, \tilde{s}_{\tilde{r}}^{\prime}\right)$ by

$s_{i}^{\prime}=1$ if $i \in \mathcal{J}, s_{i}^{\prime}=s_{i}$ if $i \in[1, r]-\mathcal{J}, \tilde{s}_{j}^{\prime}=1$ if $j \in \tilde{\mathcal{J}}, \tilde{s}_{j}^{\prime}=\tilde{s}_{j}$ if $j \in[1, \tilde{r}]-\tilde{\mathcal{J}}$.

We have $e_{6}(x)=\left(\left(y_{1}, \ldots, y_{r}\right),\left(\tilde{y}_{1}, \ldots, \tilde{y}_{\tilde{r}}\right)\right)$ where $y_{i}=\ddot{a}_{\tilde{r}+i-1} \ddot{a}_{\tilde{r}+i}^{-1}$ for $i \in[1, r]$ and $\tilde{y}_{j}=\ddot{a}_{j-1}^{-1} \ddot{a}_{j}$ for $j \in[1, \tilde{r}]$. Equivalently, $y_{i}=\dot{s}_{i}^{\prime}$ for $i \in[1, r]$ and $\tilde{y}_{j}={\dot{s_{s}}}_{j}$ for $j \in[1, \tilde{r}]$. Thus we have $e_{6}(x) \in \mathcal{Y}^{\mathcal{J}} \times \tilde{\mathcal{Y}}^{\tilde{\mathcal{J}}}$. Moreover, we have $\left(f^{\mathcal{J}} \times \tilde{f} \tilde{\mathcal{J}}\right) e_{6}(x)=$ $(1,1) \in T \times T$. It is enough to show that the $\mathcal{T}$-action on the stalk of $\overline{\mathcal{L}}_{2} \otimes \overline{\tilde{\mathcal{L}}}_{2}$ at $e_{6}(x)$ is trivial if and only if $\mathcal{L} \otimes\left(a_{0}^{-1}\right)^{*} \tilde{\mathcal{L}} \cong \overline{\mathbf{Q}}_{l}$. (The $\mathcal{T}$-equivariant structure on $\overline{\mathcal{L}}_{2} \otimes \overline{\tilde{\mathcal{L}}}_{2}$ is obtained by restricting the $\underline{B} \times \underline{\tilde{B}}$-equivariant structure in 6.2.) $\mathrm{By}$ 2.4(a) we have canonically

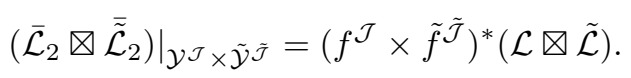

Now $\mathcal{Y}^{\mathcal{J}} \times \tilde{\mathcal{Y}}^{\tilde{\mathcal{J}}}$ is stable under the $\underline{B} \times \underline{\tilde{B}}$-action on $Z_{2} \times \tilde{Z}_{2}$ and the previous equality shows that $\left(f^{\mathcal{J}} \times \tilde{f}_{\tilde{\mathcal{J}}}\right)^{*}(\mathcal{L} \otimes \tilde{\mathcal{L}})$ has a $\underline{B} \times \underline{\tilde{B}}$-equivariant structure; this structure is unique since $\underline{B} \times \underline{\tilde{B}}$ is connected. By restriction to the subgroup $\mathcal{T}$ of $\underline{B} \times \underline{\tilde{B}}$ we obtain a $\mathcal{T}$-equivariant structure on $\left(f^{\mathcal{J}} \times \tilde{f}^{\tilde{\mathcal{J}}}\right)^{*}(\mathcal{L} \otimes \tilde{\mathcal{L}})$ and it is enough to show that the $\mathcal{T}$-action on the stalk of $\left(f^{\mathcal{J}} \times \tilde{f}^{\tilde{\mathcal{J}}}\right)^{*}(\mathcal{L} \otimes \tilde{\mathcal{L}})$ at $e_{6}(x)$ is trivial if and only if $\mathcal{L} \otimes\left(a_{0}^{-1}\right)^{*} \tilde{\mathcal{L}} \cong \overline{\mathbf{Q}}_{l}$. We define a $\underline{B} \times \underline{\tilde{B}}$-action on $T \times T$ by

$$
\left(\left(b_{i}\right),\left(\tilde{b}_{j}\right)\right):(t, \tilde{t}) \mapsto\left(k\left(b_{0}\right) t\left(\left[\mathbf{s}_{\mathcal{J}}\right] F k\left(b_{0}^{-1}\right)\right), k\left(\tilde{b}_{0}\right) \tilde{t}\left(\left[\tilde{\mathbf{s}}_{\tilde{J}}\right] F k\left(\tilde{b}_{0}^{-1}\right)\right)\right) .
$$

From the definitions we see that $f^{\mathcal{J}} \times \tilde{f}^{\tilde{\mathcal{J}}}: Z_{2} \times \tilde{Z}_{2} \rightarrow T \times T$ is compatible with the $\underline{B} \times \underline{\tilde{B}}$-actions. Moreover, $\mathcal{L} \otimes \tilde{\mathcal{L}}$ is equivariant for the $\underline{B} \times \underline{\tilde{B}}$-action on $T \times T$ as above. (In fact, it is equivariant for the action of the bigger group $B^{[0, r]} \times B^{[0, \tilde{r}]}$ given by the same formula; this follows from 1.8 using that $\mathcal{L} \in \mathcal{S}(T)^{\left[\mathbf{s}_{\mathcal{J}}\right] F}$ and $\mathcal{L} \in \mathcal{S}(T)^{\left[\tilde{\mathbf{s}}_{\tilde{\mathcal{J}}}\right] F}$.) By restriction to the subgroup $\mathcal{T}$ of $\underline{B} \times \underline{\tilde{B}}$ we obtain a $\mathcal{T}$-equivariant structure on 
$\mathcal{L} \otimes \tilde{\mathcal{L}}$. We may identify the stalk of $\left(f^{\mathcal{J}} \times \tilde{f} \tilde{\mathcal{J}}\right)^{*}(\mathcal{L} \otimes \tilde{\mathcal{L}})$ at $e_{6}(x)$ with the stalk of $\mathcal{L} \otimes \tilde{\mathcal{L}}$ at $(1,1)$ as $\mathcal{T}$-modules. Let $\chi: \mathcal{T} \rightarrow \overline{\mathbf{Q}}_{l}^{*}$ be the character by which $\mathcal{T}$ acts on the stalk of $\mathcal{L} \otimes \tilde{\mathcal{L}}$ at $(1,1)$. It is enough to show that $\chi=1$ if and only if $\mathcal{L} \otimes\left(a_{0}^{-1}\right)^{*} \tilde{\mathcal{L}} \cong \overline{\mathbf{Q}}_{l}$.

Now let $T^{\prime}$ be the subgroup of $B^{[0, r]} \times B^{[0, \tilde{r}]}$ consisting of all elements $\left(\left(t_{0}, t_{1}\right.\right.$, $\left.\left.\ldots, t_{r}\right),\left(\tilde{t}_{0}, \tilde{t}_{1}, \ldots, \tilde{t}_{\tilde{r}}\right)\right)$ with coordinates in $T$ such that $\tilde{t}_{j}=a_{j}^{-1}\left(t_{0}\right)$ for $j \in[0, \tilde{r}]$, $t_{i}=a_{\tilde{r}+i}\left(\tilde{t}_{\tilde{r}}\right)$ for $i \in[0, r]$. We have $\mathcal{T} \subset T^{\prime}$ and $\left(\left(t_{i}\right),\left(\tilde{t}_{j}\right)\right) \mapsto t_{0}$ is an isomorphism $T^{\prime} \stackrel{\sim}{\rightarrow} T$. Moreover, $\mathcal{L} \otimes \tilde{\mathcal{L}}$ is $T^{\prime}$-equivariant where $T^{\prime}$ acts on $T \times T$ by restriction of the $B^{[0, r]} \times B^{[0, \tilde{r}]}$ action. Now $T^{\prime}$ acts on $T$ by $t_{0}: t \mapsto t_{0} t F^{\prime}\left(t_{0}^{-1}\right)$ where $F^{\prime}: T \rightarrow T$, $F^{\prime}\left(t_{0}\right)=\left(a_{\tilde{r}} a_{\rho}^{-1} F\right)\left(t_{0}\right)$ is the Frobenius map for an $\mathbf{F}_{q}$-rational structure on $T$. The map $m: T \rightarrow T \times T, t \mapsto\left(t, a_{0}^{-1}(t)\right)$ is compatible with the $T^{\prime}$-actions. Hence $m^{*}(\mathcal{L} \otimes \tilde{\mathcal{L}})=\mathcal{L} \otimes\left(a_{0}^{-1}\right)^{*} \tilde{\mathcal{L}}$ is a $T^{\prime}$-equivariant local system on $T$ and the natural action of $\mathcal{T}$ at any stalk of this local system is via $\chi$. If we identify $T^{\prime}$ with $T$ as above, $\mathcal{T}$ becomes the subgroup $T^{F^{\prime}}$ of $T$. It remains to use the bijection (i)-(iv) in 1.9 , with $\mathcal{L}$ replaced by $\mathcal{L} \otimes\left(a_{0}^{-1}\right)^{*} \tilde{\mathcal{L}}$.

6.11. We assume that a satisfies $6.9(\mathrm{a})$. We show:

(a) $\mathcal{E}_{6} \cong \overline{\mathbf{Q}}_{l}$.

We write $\overline{\mathcal{L}}_{2}=\underline{\underline{\mathcal{L}}}=\otimes_{i \in[1, r]} \mathcal{F}_{i}$ as in 2.4. Similarly $\overline{\tilde{\mathcal{L}}}_{2}=\otimes_{j \in[1, \tilde{r}]} \tilde{\mathcal{F}}_{j}$ where $\tilde{\mathcal{F}}_{j}$ are local systems on $\tilde{Z}_{2}$. From the definitions we have

$$
\mathcal{E}_{6}=\otimes_{i \in[1, r]} e_{6}^{*}\left(\mathcal{F}_{i} \otimes \overline{\mathbf{Q}}_{l}\right) \otimes \otimes_{j \in[1, \tilde{r}]} e_{6}^{*}\left(\overline{\mathbf{Q}}_{l} \otimes \tilde{\mathcal{F}}_{j}\right) .
$$

It is enough to show:

(b) $e_{6}^{*}\left(\mathcal{F}_{i} \otimes \overline{\mathbf{Q}}_{l}\right) \cong \overline{\mathbf{Q}}_{l}$ for any $i \in[1, r]$;

(c) $e_{6}^{*}\left(\overline{\mathbf{Q}}_{l} \otimes \tilde{\mathcal{F}}_{j}\right) \cong \overline{\mathbf{Q}}_{l}$ for any $j \in[1, \tilde{r}]$.

We prove (b). The general case can be reduced to the case where $G$ has simply connected derived group, which we now assume. Let $\psi: Z_{2} \times \tilde{Z}_{2} \rightarrow Z_{2}$ be the projection. Let $p_{i}, f_{s_{i}}$ be as in 2.4. Let $\tilde{f}_{i}$ be the obvious map from $P_{s_{i}}$ to the quotient of $P_{s_{i}} / U_{P_{s_{i}}}$ by its derived subgroup. Then $e_{6}^{*}\left(\mathcal{F}_{i} \otimes \overline{\mathbf{Q}}_{l}\right)$ is the inverse image of a local system of rank 1 under $\tilde{f}_{i} p_{i} \psi e_{6}$ (if $i \in \mathcal{I}_{\mathbf{s}}$ ), or $f_{s_{i}} p_{i} \psi e_{6}$ (if $i \in[1, r]-\mathcal{I}_{\mathbf{s}}$ ).

It is enough to show that the image of $\tilde{f}_{i} p_{i} \psi e_{6}$ (if $i \in \mathcal{I}_{\mathbf{s}}$ ) or of $f_{s_{i}} p_{i} \psi e_{6}$ (if $\left.i \in[1, r]-\mathcal{I}_{\mathbf{s}}\right)$ is a point.

Let $\xi=\left(\left(g_{0}, g_{1}, \ldots, g_{r}\right),\left(\tilde{g}_{0}, \tilde{g}_{1}, \ldots, \tilde{g}_{\tilde{r}}\right)\right) \in X_{6}$. We have $p_{i} \psi e_{6}(\xi)=g_{i-1}^{-1} g_{i}$. We have $g_{i}^{-1} \tilde{g}_{\tilde{r}} \in U \ddot{a}_{\tilde{r}+i} U, g_{i-1}^{-1} \tilde{g}_{\tilde{r}} \in U \ddot{a}_{\tilde{r}+i-1} U$, hence $g_{i-1}^{-1} g_{i} \in u_{1} \ddot{a}_{\tilde{r}+i-1} u_{2} \ddot{a}_{\tilde{r}+i}^{-1} u_{3}$ with $u_{1}, u_{2}, u_{3}$ in $U$. Moreover, $g_{i-1}^{-1} g_{i} \in P_{s_{i}}$.

Case 1. Assume that $i \in \mathcal{I}_{\mathbf{s}}$ and $a_{\tilde{r}+i-1}=a_{\tilde{r}+i}$. Then $\ddot{a}_{\tilde{r}+i-1}=\ddot{a}_{\tilde{r}+i}^{-1}$ and $\ddot{a}_{\tilde{r}+i-1} u_{2} \ddot{a}_{\tilde{r}+i}^{-1}=\ddot{a}_{\tilde{r}+i-1} u_{2} \ddot{a}_{\tilde{r}+i-1}^{-1} \in P_{s_{i}}$ is unipotent. Hence $g_{i-1}^{-1} g_{i}$ is a product of three unipotent elements of $P_{s_{i}}$ so that $\tilde{f}_{i}\left(g_{i-1}^{-1} g_{i}\right)=1$.

Case 2. Assume that $i \in \mathcal{I}_{\mathbf{s}}$ and $a_{\tilde{r}+i-1}=s_{i} a_{\tilde{r}+i}$. Then $\ddot{a}_{\tilde{r}+i}=\dot{s}_{i}^{-1} \ddot{a}_{\tilde{r}+i-1}^{-1}$ and

$$
\ddot{a}_{\tilde{r}+i-1} u_{2} \ddot{a}_{\tilde{r}+i}^{-1}=\ddot{a}_{\tilde{r}+i-1} u_{2} \ddot{a}_{\tilde{r}+i-1}^{-1} \dot{s}_{i}=u_{2}^{\prime} \dot{s}_{i}
$$

where $u_{2}^{\prime} \in G$ is unipotent. Thus $g_{i-1}^{-1} g_{i}=u_{1} u_{2}^{\prime} \dot{s}_{i} u_{3}$. Since $u_{1} u_{2}^{\prime} \dot{s}_{i} u_{3}, u_{1}, \dot{s}_{i}, u_{3}$ belong to $P_{s_{i}}$ we see that $u_{2}^{\prime}$ is a unipotent element of $P_{s_{i}}$. Note also that $\dot{s}_{i}$ belongs to the derived subgroup of $P_{s_{i}}$. We see that $\tilde{f}_{i}\left(g_{i-1}^{-1} g_{i}\right)=1$.

Case 3. Assume that $i \notin \mathcal{I}_{\mathbf{s}}$. By our assumption we have $a_{\tilde{r}+i-1}=s_{i} a_{\tilde{r}+i}$. Then $\ddot{a}_{\tilde{r}+i}=\dot{s}_{i}^{-1} \ddot{a}_{\tilde{r}+i-1}^{-1}$ and as before we have $g_{i-1}^{-1} g_{i}=u_{1} u_{2}^{\prime} \dot{s}_{i} u_{3}$ where $u_{2}^{\prime}$ belongs to the unipotent group $P_{s_{i}} \cap \ddot{a}_{\tilde{r}+i-1} U \ddot{a}_{\tilde{r}+i-1}^{-1}$. This unipotent group is normalized by 
T. By [Bo, 14.4] we have $P_{s_{i}} \cap \ddot{a}_{\tilde{r}+i-1} U \ddot{a}_{\tilde{r}+i-1}^{-1}=U_{1} U_{2} \ldots U_{n}$ where $U_{1}, U_{2}, \ldots, U_{n}$ are the connected one-dimensional unipotent subgroups of $P_{s_{i}} \cap \ddot{a}_{\tilde{r}+i-1} U \ddot{a}_{\tilde{r}+i-1}^{-1}$ normalized by $T$, in any order. Thus any of $U_{1}, U_{2}, \ldots, U_{n}$ is either $y_{s_{i}}(\mathbf{k})$ or else is contained in $U$; moreover, we can assume that $U_{1}, U_{2}, \ldots, U_{n-1}$ are contained in $U$ and $U_{n}=y_{s_{i}}(\mathbf{k})$. Thus $u_{2}^{\prime} \in U y_{s_{i}}(\mathbf{k})$ and

$$
g_{i-1}^{-1} g_{i} \in U y_{s_{i}}(\mathbf{k}) \dot{s}_{i} U=U \dot{s}_{i} x_{s_{i}}(\mathbf{k}) U=U \dot{s}_{i} U .
$$

Note that $f_{s_{i}}\left(g_{i-1}^{-1} g_{i}\right)=1$.

We see that in Cases 1 and 2 we have $\tilde{f}_{i} p_{i} \psi e_{6}(\xi)=1$ for any $\xi \in X_{6}$. In Case 3 we have $f_{s_{i}} p_{i} \psi e_{6}(\xi)=1$ for any $\xi \in X_{6}$. This completes the proof of (b).

The proof of (c) is entirely similar.

6.12. We set $X_{-1}=X, \mathcal{E}_{-1}=\mathcal{E}$. Let $\delta_{i}=\operatorname{dim} X_{i}(i \in[-1,8])$. For $i \in[-1,8]$ we show:

(a) Assume that a satisfies 6.9(a); if $n \neq 2 \delta_{i}$ or if $\tilde{\mathcal{L}} ¥ a_{0}^{*} \check{\mathcal{L}}$, then $H_{c}^{n}\left(X_{i}, \mathcal{E}_{i}\right)^{\Gamma \times \mathcal{T}}$ $=0$; if $\tilde{\mathcal{L}}=w^{*} \check{\mathcal{L}}$ where $w \in W$ and $w^{*} \mathcal{L} \cong a_{0}^{*} \mathcal{L}$, then $H_{c}^{2 \delta_{i}}\left(X_{i}, \mathcal{E}_{i}\right)^{\Gamma \times \mathcal{T}}\left(\delta_{i}\right)=\overline{\mathbf{Q}}_{l}$ canonically. Assume that a does not satisfy 6.9(a); then $H_{c}^{n}\left(X_{i}, \mathcal{E}_{i}\right)^{\Gamma \times \mathcal{T}}=0$ for all $n$.

Here $\left(\delta_{i}\right)$ is a Tate twist, The upper index denotes $\Gamma \times \mathcal{T}$-invariants (the action of $\Gamma \times \mathcal{T}$ comes from the $\Gamma \times \mathcal{T}$-equivariant structure of $\mathcal{E}_{i}$ ).

Let $P_{i}$ be the statement of (a). From 6.4 we see that the statements $P_{0}, P_{1}$ are equivalent and that the statements $P_{2}, P_{3}, \ldots, P_{8}$ are equivalent. From 6.4 we see also that $H_{c}^{n}\left(X_{2}, \mathcal{E}_{2}\right)=H_{c}^{n}\left(X_{1}, \mathcal{E}_{1}\right)^{\Gamma}$ and that $H_{c}^{n}\left(X_{-1}, \mathcal{E}_{-1}\right)=H_{c}^{n}\left(X_{0}, \mathcal{E}_{0}\right)^{\mathcal{T}}$ for any $n$. Since $\Gamma$ acts trivially on any stalk of $\mathcal{E}_{2}$ (see $6.5(\mathrm{c})$ ) and $\mathcal{T}$ acts trivially on any stalk of $\mathcal{E}_{-1}$ (see $6.5(\mathrm{~b})$ ), it follows that $H_{c}^{n}\left(X_{2}, \mathcal{E}_{2}\right)^{\Gamma}=H_{c}^{n}\left(X_{1}, \mathcal{E}_{1}\right)^{\Gamma}$ and that $H_{c}^{n}\left(X_{-1}, \mathcal{E}_{-1}\right)^{\mathcal{T}}=H_{c}^{n}\left(X_{0}, \mathcal{E}_{0}\right)^{\mathcal{T}}$ for any $n$. This shows that the statements $P_{1}, P_{2}$ are equivalent and the statements $P_{-1}, P_{0}$ are equivalent. We see that the statements $P_{-1}, P_{0}, \ldots, P_{8}$ are all equivalent. Thus it is enough to show that $P_{8}$ holds.

Assume first that a does not satisfy 6.9(a). Then the result follows from 6.7(a) or $6.8(\mathrm{a})$.

Next we assume that a satisfies $6.9(\mathrm{a})$. Let $\bar{x}=b_{8}\left(b_{7}(x)\right) \in X_{8}$ where $x \in X_{6}$ is as in 6.10. We can write 6.11 (a) in the form $b_{7}^{*} b_{8}^{*} \mathcal{E}_{8} \cong \overline{\mathbf{Q}}_{l}$. Since $b_{8}$ is an isomorphism and $b_{7}$ is a principal $U^{[0, r]} \times U^{[0, \tilde{r}]}$-bundle, it follows that $\mathcal{E}_{8} \cong \overline{\mathbf{Q}}_{l}$. Using this and 6.9 (b) we see that

$$
H_{c}^{n}\left(X_{8}, \mathcal{E}_{8}\right)=0 \text { for } i \neq 2 \delta_{8}, H_{c}^{2 \delta_{8}}\left(X_{8}, \mathcal{E}_{8}\right)\left(\delta_{8}\right)=\mathcal{E}_{8, \bar{x}}
$$

where $\mathcal{E}_{8, \bar{x}}$ is the stalk of $\mathcal{E}_{8}$ at $\bar{x}$. Moreover, the last equality is compatible with the natural $\mathcal{T}$-actions (coming from the $\mathcal{T}$-equivariant structure of $\mathcal{E}_{8}$ ). Now $\mathcal{E}_{8, \bar{x}}$ may be canonically identified with the stalk $\mathcal{E}_{6, x}$ of $\mathcal{E}_{6}$ at $x$. Thus we have $H_{c}^{2 \delta_{8}}\left(X_{8}, \mathcal{E}_{8}\right)\left(\delta_{8}\right)=\mathcal{E}_{6, x}$, compatibly with the $\mathcal{T}$-actions. Using 6.10 (a) we see that the $\mathcal{T}$-action on $H_{c}^{2 \delta_{8}}\left(X_{8}, \mathcal{E}_{8}\right)$ is trivial if and only if $\mathcal{L} \otimes\left(a_{0}^{-1}\right)^{*} \tilde{\mathcal{L}} \cong \overline{\mathbf{Q}}_{l}$. Taking $\mathcal{T}$-invariants we see that

$$
\begin{aligned}
& H_{c}^{2 \delta_{8}}\left(X_{8}, \mathcal{E}_{8}\right)^{\mathcal{T}}\left(\delta_{8}\right)=\mathcal{E}_{6, x} \text { if } \mathcal{L} \otimes\left(a_{0}^{-1}\right)^{*} \tilde{\mathcal{L}} \cong \overline{\mathbf{Q}}_{l} ; \\
& H_{c}^{2 \delta_{8}}\left(X_{8}, \mathcal{E}_{8}\right)^{\mathcal{T}}=0 \text { if } \mathcal{L} \otimes\left(a_{0}^{-1}\right)^{*} \tilde{\mathcal{L}} \neq \overline{\mathbf{Q}}_{l} .
\end{aligned}
$$

By $6.5(\mathrm{c}), \Gamma$ acts trivially on $H_{c}^{2 \delta_{8}}\left(X_{8}, \mathcal{E}_{8}\right)$ hence

$H_{c}^{2 \delta_{8}}\left(X_{8}, \mathcal{E}_{8}\right)^{\Gamma \times \mathcal{T}}=H_{c}^{2 \delta_{8}}\left(X_{8}, \mathcal{E}_{8}\right)^{\mathcal{T}}$.

We see that the first assertion in (a) for $i=8$ holds. It remains to prove that, if $\tilde{\mathcal{L}}=w^{*} \check{\mathcal{L}}$ where $w \in W$ and $w^{*} \mathcal{L} \cong a_{0}^{*} \mathcal{L}$, then $\mathcal{E}_{6, x}=\overline{\mathbf{Q}}_{l}$ canonically. By the 
proof of $6.10(\mathrm{a}), \mathcal{E}_{6, x}$ is canonically isomorphic to the stalk of $\mathcal{L} \otimes\left(a_{0}^{-1}\right)^{*} \tilde{\mathcal{L}}$ at 1 ; that is, to the stalk of $\mathcal{L} \otimes\left(w a_{0}^{-1}\right)^{*} \check{\mathcal{L}}$ at 1 . The stalk of $\left(w a_{0}^{-1}\right)^{*} \breve{\mathcal{L}}$ at 1 is the same as the stalk of $\check{\mathcal{L}}$ at $w a_{0}(1)=1$. Thus $\mathcal{E}_{6, x}$ is canonically isomorphic to the stalk of $\mathcal{L} \otimes \check{\mathcal{L}}=\overline{\mathbf{Q}}_{l}$ at 1 . Thus $P_{8}$ holds. We see that (a) holds for $i \in[-1,8]$.

6.13. We now write $X_{\mathbf{a}}, \mathcal{E}_{\mathbf{a}}, e_{\mathbf{a}}$ instead of $X, \mathcal{E}, e$ in 6.1 . We identify $X_{\mathbf{a}}$ with its image under the imbedding $e_{\mathbf{a}}$. Let $\bar{X}_{\mathbf{a}}$ be the closure of $X_{\mathbf{a}}$ in $Z \times \tilde{Z}$. Recall that $\mathcal{E}_{\mathbf{a}}=e_{\mathbf{a}}^{*}(\overline{\mathcal{L}} \otimes \overline{\tilde{\mathcal{L}}})$. Let $\bar{e}_{\mathbf{a}}: \bar{X}_{\mathbf{a}} \rightarrow Z \times \tilde{Z}$ by the inclusion. By $6.9(\mathrm{c}), X_{\mathbf{a}}$ (hence also $\left.\bar{X}_{\mathbf{a}}\right)$ has pure dimension $N_{\mathbf{a}}$ and $\operatorname{dim}\left(\bar{X}_{\mathbf{a}}-X_{\mathbf{a}}\right)<N_{\mathbf{a}}$. Hence the natural map

$$
H_{c}^{2 N_{\mathbf{a}}}\left(X_{\mathbf{a}}, e_{\mathbf{a}}^{*}(\overline{\mathcal{L}} \otimes \overline{\tilde{\mathcal{L}}})\right) \rightarrow H_{c}^{2 N_{\mathbf{a}}}\left(\bar{X}_{\mathbf{a}}, \bar{e}_{\mathbf{a}}^{*}(\overline{\mathcal{L}} \otimes \overline{\tilde{\mathcal{L}}})\right)
$$

(induced by the open imbedding $X_{\mathbf{a}} \subset \bar{X}_{\mathbf{a}}$ ) is an isomorphism. Let

$$
\xi_{\mathbf{a}}^{\prime}: H_{c}^{2 N_{\mathbf{a}}}(Z \times \tilde{Z},(\overline{\mathcal{L}} \otimes \overline{\tilde{\mathcal{L}}})) \rightarrow H_{c}^{2 N_{\mathbf{a}}}\left(X_{\mathbf{a}}, e_{\mathbf{a}}^{*}(\overline{\mathcal{L}} \otimes \overline{\tilde{\mathcal{L}}})\right)
$$

be the linear map obtained by composing the linear map

$$
H_{c}^{2 N_{\mathbf{a}}}(Z \times \tilde{Z},(\overline{\mathcal{L}} \otimes \overline{\tilde{\mathcal{L}}})) \rightarrow H_{c}^{2 N_{\mathbf{a}}}\left(\bar{X}_{\mathbf{a}}, \bar{e}_{\mathbf{a}}^{*}(\overline{\mathcal{L}} \otimes \overline{\tilde{\mathcal{L}}})\right)
$$

with the inverse of (a). By taking $\Gamma$-invariants and applying a Tate twist we obtain from $\xi_{\mathbf{a}}^{\prime}$ a linear map

$$
\xi_{\mathbf{a}}: H_{c}^{2 N_{\mathbf{a}}}(Z \times \tilde{Z},(\overline{\mathcal{L}} \otimes \overline{\tilde{\mathcal{L}}}))^{\Gamma}\left(N_{\mathbf{a}}\right) \rightarrow H_{c}^{2 N_{\mathbf{a}}}\left(X_{\mathbf{a}}, e_{\mathbf{a}}^{*}(\overline{\mathcal{L}} \otimes \overline{\tilde{\mathcal{L}}})\right)^{\Gamma}\left(N_{\mathbf{a}}\right) .
$$

6.14. Let $\mathcal{A}$ be the set of all $\mathbf{a}$ as in 6.1 . Note that the subvarieties $X_{\mathbf{a}}(\mathbf{a} \in \mathcal{A})$ form a partition of $Z \times \tilde{Z}$.

(a) For any $n \in \mathbf{Z}$, the linear map

$$
\xi_{n}: H_{c}^{2 n}(Z \times \tilde{Z}, \overline{\mathcal{L}} \otimes \overline{\tilde{\mathcal{L}}})^{\Gamma}(n) \rightarrow \oplus_{\mathbf{a} \in \mathcal{A} ; N_{\mathbf{a}}=n} H_{c}^{2 n}\left(X_{\mathbf{a}}, e_{\mathbf{a}}^{*}(\overline{\mathcal{L}} \otimes \overline{\tilde{\mathcal{L}}})\right)^{\Gamma}(n)
$$

whose components are the maps $\xi_{\mathbf{a}}\left(\mathbf{a} \in \mathcal{A}, N_{\mathbf{a}}=n\right)$, is an isomorphism. Moreover, $H_{c}^{n^{\prime}}(Z \times \tilde{Z}, \overline{\mathcal{L}} \otimes \overline{\tilde{\mathcal{L}}})^{\Gamma}=0$ for any odd $n^{\prime}$.

The proof is almost identical to that of [L4, 2.7]. (We use the fact that

$$
H_{c}^{m}\left(X_{\mathbf{a}}, e_{\mathbf{a}}^{*}(\overline{\mathcal{L}} \otimes \overline{\tilde{\mathcal{L}}})\right)^{\Gamma}=0
$$

for any $\mathbf{a} \in \mathcal{A}$ and any $m \neq 2 N_{\mathbf{a}}$; this follows from 6.12 (a) for $i=-1$; note that, in view of $6.5(\mathrm{~b}), 6.12(\mathrm{a})$ for $i=-1$ remains valid if $\Gamma \times \mathcal{T}$-invariants are replaced by $\Gamma$-invariants.)

From (a) and 6.12(a) for $i=-1$ we see that the following holds.

(b) If for any $w \in W$ we have $\tilde{\mathcal{L}} ¥ w^{*} \check{\mathcal{L}}$, then $H_{c}^{m}(Z \times \tilde{Z}, \overline{\mathcal{L}} \otimes \overline{\tilde{\mathcal{L}}})^{\Gamma}=0$ for any $m \in \mathbf{Z}$

Now assume that $\tilde{\mathcal{L}}=w^{*} \check{\mathcal{L}}$ for some $w \in W$. Let

$\mathcal{A}_{w}=\left\{\mathbf{a}=\left(a_{0}, a_{1}, \ldots, a_{\rho}\right) \in \mathcal{A} ; \mathbf{a}\right.$ satisfies $\left.6.9(\mathrm{a}), a_{0}^{*} \mathcal{L} \cong w^{*} \mathcal{L}.\right\}$.

Note that for $w=1$ we have $\mathcal{A}_{1}=\mathcal{A}(W, \mathbf{c}, \mathcal{L}, \mathbf{s}, \tilde{\mathbf{s}})$; see 5.9 .

By 6.12(a) for $i=-1$, the summand in the target of $\xi_{n}$ corresponding to a is canonically $\overline{\mathbf{Q}}_{l}$ if $\mathbf{a} \in \mathcal{A}_{w}$ and is 0 if $\mathbf{a} \notin \mathcal{A}_{w}$. Hence for any $\mathbf{a} \in \mathcal{A}_{w}$ with $N_{\mathbf{a}}=n$ there is a unique element $b_{\mathbf{a}}^{w} \in H_{c}^{2 n}(Z \times \tilde{Z}, \overline{\mathcal{L}} \otimes \overline{\tilde{\mathcal{L}}})^{\Gamma}(n)$ such that $\xi_{n}\left(b_{\mathbf{a}}^{w}\right)$ is contained in the summand corresponding to a and, as an element of that summand, it corresponds to $1 \in \overline{\mathbf{Q}}_{l}$. Moreover,

(c) $\left\{b_{\mathbf{a}}^{w} ; \mathbf{a} \in \mathcal{A}_{w}, N_{\mathbf{a}}=n\right\}$ is a $\overline{\mathbf{Q}}_{l}$-basis of $H_{c}^{2 n}(Z \times \tilde{Z}, \overline{\mathcal{L}} \otimes \overline{\tilde{\mathcal{L}}})^{\Gamma}(n)$.

Taking $w=1$ and taking direct sum over $n$ we obtain

(d) $\left\{b_{\mathbf{a}}^{1} ; \mathbf{a} \in \mathcal{A}(W, \mathbf{c}, \mathcal{L}, \mathbf{s}, \tilde{\mathbf{s}})\right\}$ is a $\overline{\mathbf{Q}}_{l}$-basis of $\oplus_{n} H_{c}^{2 n}(Z \times \tilde{Z}, \overline{\mathcal{L}} \otimes \overline{\tilde{\mathcal{L}}})^{\Gamma}(n)$. 
6.15. More generally, if $J$ is as in 3.1 , we set $\mathcal{A}_{J}=\left\{\mathbf{a}=\left(a_{0}, a_{1}, \ldots, \alpha_{\rho}\right) \in \mathcal{A} ; a_{0} \in\right.$ $\left.W_{J}\right\}$. Let

$$
(Z \times \tilde{Z})_{J}=\left\{\left(\left(B_{0}, B_{1}, \ldots, B_{r}\right),\left(\tilde{B}_{0}, \tilde{B}_{1}, \ldots, \tilde{B}_{\tilde{r}}\right)\right) \in Z \times \tilde{Z} ; P_{B_{0}, J}=P_{\tilde{B}_{0}, J}\right\} .
$$

Note that the subvarieties $X_{\mathbf{a}}\left(\mathbf{a} \in \mathcal{A}_{J}\right)$ form a partition of $(Z \times \tilde{Z})_{J}$. As in 6.14 we see that for any $n \in \mathbf{Z}$ we have an isomorphism

$$
H_{c}^{2 n}\left((Z \times \tilde{Z})_{J}, \overline{\mathcal{L}} \otimes \overline{\tilde{\mathcal{L}}}\right)^{\Gamma}(n) \stackrel{\sim}{\rightarrow} \oplus_{\mathbf{a} \in \mathcal{A}_{J} ; N_{\mathbf{a}}=n} H_{c}^{2 n}\left(X_{\mathbf{a}}, e_{\mathbf{a}}^{*}(\overline{\mathcal{L}} \otimes \overline{\tilde{\mathcal{L}}})\right)^{\Gamma}(n)
$$

Moreover,

$$
H_{c}^{n^{\prime}}\left((Z \times \tilde{Z})_{J}, \overline{\mathcal{L}} \otimes \overline{\tilde{\mathcal{L}}}\right)^{\Gamma}=0 \text { for any odd } n^{\prime} .
$$

As in 6.14 we see that the following holds:

(c) If for any $w \in W_{J}$ we have $\tilde{\mathcal{L}} \not w^{*} \check{\mathcal{L}}$, then $H_{c}^{m}\left((Z \times \tilde{Z})_{J}, \overline{\mathcal{L}} \otimes \overline{\tilde{\mathcal{L}}}\right)^{\Gamma}=0$ for any $m \in \mathbf{Z}$.

Define $h:(Z \times \tilde{Z}) \rightarrow \mathcal{P}_{J}$ by $\left(\left(B_{0}, B_{1}, \ldots, B_{r}\right),\left(\tilde{B}_{0}, \tilde{B}_{1}, \ldots, \tilde{B}_{\tilde{r}}\right)\right) \mapsto P_{B_{0}, J}=$ $P_{\tilde{B}_{0}, J}$. We have

$$
H_{c}^{m}\left((Z \times \tilde{Z})_{J}, \overline{\mathcal{L}} \otimes \overline{\tilde{\mathcal{L}}}\right)=H_{c}^{m}\left(\mathcal{P}_{J}, h_{!}(\overline{\mathcal{L}} \otimes \overline{\tilde{\mathcal{L}}})\right)=H_{c}^{m}\left(\mathcal{P}_{J},\left(\Upsilon_{!}^{\mathbf{s}} \overline{\mathcal{L}}\right) \otimes\left(\Upsilon_{!}^{\tilde{s}} \overline{\tilde{\mathcal{L}}}\right)\right) .
$$

(Notation of 3.2.) From 2.13 we see that

$$
\Upsilon_{!}^{\mathbf{s}} \overline{\mathcal{L}}=\bar{\Upsilon}_{!}^{\mathbf{s}} \overline{\mathcal{L}}^{\sharp}
$$

(notation of 3.2). By the decomposition theorem [BBD, $\bar{\Upsilon}_{!}^{\mathrm{s}} \overline{\mathcal{L}}^{\sharp} \cong \oplus_{j}^{p} H^{j}\left(\bar{\Upsilon}_{!}^{\mathrm{s}} \overline{\mathcal{L}}^{\sharp}\right)[-j]$. Hence $\Upsilon_{!}^{\mathrm{s}} \overline{\mathcal{L}} \cong \oplus_{j}^{p} H^{j}\left(\Upsilon_{!}^{\mathrm{s}} \overline{\mathcal{L}}\right)[-j]$. Similarly, $\Upsilon_{!}^{\tilde{\mathbf{S}}} \overline{\tilde{\mathcal{L}}} \cong \oplus_{j}^{p} H^{\tilde{j}}\left(\Upsilon_{!}^{\tilde{\mathrm{s}}} \overline{\tilde{\mathcal{L}}}\right)[-\tilde{j}]$. We see that

(e) $\quad H_{c}^{m}\left((Z \times \tilde{Z})_{J}, \overline{\mathcal{L}} \otimes \overline{\tilde{\mathcal{L}}}\right)=\oplus_{j, j^{\prime}} H_{c}^{m-j-\tilde{j}}\left(\mathcal{P}_{J},{ }^{p} H^{j}\left(\Upsilon_{!}^{\mathbf{s}} \overline{\mathcal{L}}\right) \otimes{ }^{p} H^{\tilde{j}}\left(\Upsilon_{!}^{\tilde{s}} \overline{\tilde{\mathcal{L}}}\right)\right)$

(compatibly with the $\Gamma$-actions).

Proposition 6.16. Let $J \subset \mathbf{I}$. Let $A$ be a simple object of $\mathcal{M}_{\Gamma}\left(\mathcal{P}_{J}\right)$. Assume that $A \dashv_{\Gamma}{ }^{p} H^{j}\left(\Upsilon_{!}^{\mathbf{s}} \overline{\mathcal{L}}\right)$ and $A \dashv_{\Gamma}{ }^{p} H^{j^{\prime}}\left(\Upsilon_{!}^{\tilde{\mathrm{s}}} \overline{\mathcal{L}}\right)$ where $j, j^{\prime} \in \mathbf{Z}$. Then $\tilde{\mathcal{L}} \cong w^{*} \check{\mathcal{L}}$ for some $w \in W_{J}$ and $j=j^{\prime} \bmod 2$.

Using $6.15(\mathrm{~d})$ and the fact that $\bar{\Upsilon}^{\mathbf{s}}$ is proper we see that

$$
\mathfrak{D}\left(\Upsilon_{!}^{\mathbf{s}} \overline{\mathcal{L}}\right)=\bar{\Upsilon}_{!}^{\mathrm{s}} \mathfrak{D}\left(I C\left(\bar{Z}^{\mathbf{s}}, \overline{\mathcal{L}}\right)\right)=\bar{\Upsilon}_{!}^{\mathbf{s}}\left(I C\left(\bar{Z}^{\mathbf{s}}, \overline{\mathcal{L}}\right)\right)[2 r]=\Upsilon_{!}^{\mathbf{s}} \overline{\mathcal{L}}[2 r]
$$

Hence $\mathfrak{D}\left({ }^{p} H^{j}\left(\Upsilon_{!}^{\mathrm{s}} \overline{\mathcal{L}}\right)\right)={ }^{p} H^{-j+2 r}\left(\Upsilon_{!}^{\mathrm{s}} \overline{\mathcal{L}}\right)$. We see that $\mathfrak{D}(A) \dashv_{\Gamma}{ }^{p} H^{-j+2 r}\left(\Upsilon_{!}^{\mathrm{s}} \overline{\mathcal{L}}\right)$. By 1.6(a) we have $\operatorname{dim} H_{c}^{0}\left(\mathcal{P}_{J}, \mathfrak{D}(A) \otimes A\right)^{\Gamma}=1$. Since $\mathfrak{D}(A)$ (resp. $A$ ) is a direct summand of ${ }^{p} H^{-j+2 r}\left(\Upsilon_{!}^{\mathrm{s}} \overline{\mathcal{L}}\right)\left(\right.$ resp. $\left.{ }^{p} H^{j^{\prime}}\left(\Upsilon_{!}^{\tilde{\mathrm{s}}} \overline{\tilde{\mathcal{L}}}\right)\right)$ we see that

$$
H_{c}^{0}\left(\mathcal{P}_{J},{ }^{p} H^{-j+2 r}\left(\Upsilon_{!}^{\mathbf{s}} \overline{\mathcal{L}}\right) \otimes{ }^{p} H^{j^{\prime}}\left(\Upsilon_{!}^{\tilde{s}} \overline{\tilde{\mathcal{L}}}\right)\right)^{\Gamma} \neq 0 .
$$

Using $6.15(\mathrm{e})$ we see that $H_{c}^{-j+2 r+j^{\prime}}\left((Z \times \tilde{Z})_{J}, \overline{\mathcal{L}} \otimes \overline{\tilde{\mathcal{L}}}\right)^{\Gamma} \neq 0$. Using 6.15(b),(c) we see that the proposition holds.

6.17. In the case where $J=\mathbf{I}$, the first assertion of 6.16 reduces to the disjointness theorem [DL, 6.2, 6.3] (we use also the equivalence of 3.2(i), 3.2(vi)). 
6.18. In this subsection we assume that $\tilde{\mathcal{L}}=\check{\mathcal{L}}$ and

(a) any $s \in \mathbf{I}$ is in the $\mathbf{c}$-orbit of some $s_{i}(i \in[1, r])$.

Since $\operatorname{dim}(Z \times \tilde{Z})=\rho$, we have $H_{c}^{i}(Z \times \tilde{Z}, \overline{\mathcal{L}} \otimes \tilde{\tilde{\mathcal{L}}})=0$ for $i<2 \rho$. We show:

(b) $\operatorname{dim} H_{c}^{2 \rho}(Z \times \tilde{Z}, \overline{\mathcal{L}} \otimes \overline{\tilde{\mathcal{L}}})^{\Gamma}=n_{\mathcal{L}}$ where $n_{\mathcal{L}}$ is 1 if $R=R_{\mathcal{L}}$ and 0 if $R \neq R_{\mathcal{L}}$.

By 6.14(c) we have $\operatorname{dim} H_{c}^{2 \rho}(Z \times \tilde{Z}, \overline{\mathcal{L}} \otimes \overline{\tilde{\mathcal{L}}})^{\Gamma}=|\mathfrak{A}|$ where $\mathfrak{A}=\left\{\mathbf{a} \in \mathcal{A}_{1} ; N_{\mathbf{a}}=\rho\right\}$.

Note that $\mathfrak{A}$ is the set of all sequences $\mathbf{a}=\left(a_{0}, a_{1}, \ldots, a_{\rho}\right)$ in $W$ such that

$a_{j-1}^{-1} a_{j} \in\left\{1, \tilde{s}_{j}\right\}$ for $j \in \mathcal{I}_{\tilde{\mathbf{s}}}$;

$a_{j-1}^{-1} a_{j}=\tilde{s}_{j}$ for $j \in[1, \tilde{r}]-\mathcal{I}_{\tilde{\mathbf{s}}}$

$a_{\tilde{r}+i} a_{\tilde{r}+i-1}^{-1} \in\left\{1, s_{i}\right\}$ for $i \in \mathcal{I}_{\mathbf{s}}$;

$a_{\tilde{r}+i} a_{\tilde{r}+i-1}^{-1}=s_{i}$ for $i \in[1, r]-\mathcal{I}_{\mathbf{s}}$;

if $h \in[1, \tilde{r}]$, then $a_{h-1} \leq a_{h} \geq a_{h-1} \tilde{s}_{h}$;

if $h \in[1, r]$, then $a_{\tilde{r}+h-1} \leq a_{\tilde{r}+h} \geq s_{h} a_{\tilde{r}+h-1}$;

$a_{\rho}=\mathbf{c}\left(a_{0}\right)$

$a_{0}^{*} \mathcal{L} \cong \mathcal{L}$.

If $\mathbf{a} \in \mathfrak{A}$, then $l\left(a_{0}\right) \leq l\left(a_{1}\right) \leq \cdots \leq l\left(a_{\rho}\right)=l\left(a_{0}\right)$ hence $l\left(a_{0}\right)=l\left(a_{1}\right)=\cdots=$ $l\left(a_{\rho}\right)$ and since $a_{0} \leq a_{1} \leq \cdots \leq a_{\rho}$, we have $a_{0}=a_{1}=\cdots=a_{\rho}$. This forces $\mathcal{I}_{\tilde{\mathbf{s}}}=[1, \tilde{r}]$. Thus, $s_{1}, s_{1} s_{2} s_{1}, \ldots, s_{1} s_{2} \ldots s_{r} \ldots s_{2} s_{1}$ are in $W_{\mathcal{L}}$ hence $s_{i} \in W_{\mathcal{L}}$ for $i \in[1, r]$. Using $([\mathbf{s}] F)^{*} \mathcal{L} \cong \mathcal{L}$ and $[\mathbf{s}] \in W_{\mathcal{L}}$ we deduce $F^{*} \mathcal{L} \cong \mathcal{L}$. Hence if $i \in[1, r]$, then $\mathbf{c}^{n}\left(s_{i}\right) \in W_{\mathcal{L}}$ for any $n \geq 1$. Using (a) we deduce that $\mathbf{I} \subset W_{\mathcal{L}}$ hence $W=W_{\mathcal{L}}$ and $R=R_{\mathcal{L}}$.

If $R=R_{\mathcal{L}}$, then $\mathfrak{A}$ is in bijection with the set

$$
\mathfrak{A}^{\prime}=\left\{a_{0} \in W ; a_{0}=\mathbf{c}\left(a_{0}\right), a_{0} \tilde{s}_{h}<a_{0} \text { for } h \in[1, \tilde{r}], s_{h} a_{0}<a_{0} \text { for } h \in[1, r]\right\} .
$$

We set $I^{\prime}=\left\{s \in \mathbf{I} ; s=s_{h}\right.$ for some $\left.h \in[1, r]\right\}, I^{\prime \prime}=\left\{s \in \mathbf{I} ; s=\tilde{s}_{h}\right.$ for some $h \in$ $[1, \tilde{r}]\}$. We see that

$$
\mathfrak{A}^{\prime}=\left\{a_{0} \in W ; I^{\prime} \subset L_{a_{0}}, I^{\prime \prime} \subset R_{a_{0}}, a_{0}=\mathbf{c}\left(a_{0}\right)\right\}
$$

where for $w \in W$ we set $L_{w}=\{s \in \mathbf{I} ; s w<w\}, R_{w}=\{s \in \mathbf{I} ; w s<w\}$.

For $a_{0}$ such that $a_{0}=\mathbf{c}\left(a_{0}\right)$, the set $L_{a_{0}}$ is c-stable; hence the condition $I^{\prime} \subset L_{a_{0}}$ is equivalent to $I^{\prime} \cup \mathbf{c}\left(I^{\prime}\right) \cup \mathbf{c}^{2}\left(I^{\prime}\right) \cup \ldots \subset L_{a_{0}}$; that is, (by (a)) to $\mathbf{I}=L_{a_{0}}$. We see that $\mathfrak{A}^{\prime}$ has exactly one element: $w_{\mathbf{I}}$. This proves (b).

\section{THE VARIETY $X$}

7.1. In this section we study the variety

$$
X=\left\{\left(B^{\prime}, g\right) \in \mathcal{B} \times G ; g^{-1} F(g) \in U_{B^{\prime}}\right\} .
$$

Note that $X$ is an étale covering of the smooth connected variety $X^{\prime}=\left\{\left(B^{\prime}, u\right) \in\right.$ $\left.\mathcal{B} \times G ; u \in U_{B^{\prime}}\right\}$ of dimension via the map $\rho_{1}: X \rightarrow X^{\prime},\left(B^{\prime}, g\right) \mapsto\left(B^{\prime}, g^{-1} F(g)\right)$. Since $\operatorname{dim} X^{\prime}=2 d$ where $d=\operatorname{dim} \mathcal{B}$, we see that $X$ is smooth of pure dimension $2 d$. Here is one of the main results of this section.

Proposition 7.2. If $G$ is simply connected, then $X$ is connected.

The proof is given in 7.16. Note that $X$ is not necessarily connected without the assumption that $G$ is simply connected. 
7.3. Let $w \in W$. Let $i_{w}: \mathcal{B}_{w} \rightarrow \mathcal{B}$ be the inclusion. Let $A^{\prime} \in \mathbb{S}\left(\mathcal{B}_{w}\right)$. Let $A$ be a simple object of $\mathcal{M}_{\Gamma}(\mathcal{B})$ such that $A \dashv_{\Gamma}{ }^{p} H^{\cdot}\left(i_{w} A^{\prime}\right)$. We show:

(a)

$$
A \in \mathbb{S}(\mathcal{B})
$$

We argue by induction on $l(w)$. If $l(w)=0$, then $i_{w !} A^{\prime} \in \mathbb{S}(\mathcal{B})$ and the result is clear. We now assume that $l(w)>0$. We have $A=\mathcal{L}_{w}[l(w)]$ where $\mathcal{L} \in \mathcal{S}(T)^{w F}$. Let $K=\mathcal{L}_{w}^{\sharp}[l(w)]$. We have $K \in \mathbb{S}(\mathcal{B})$. Assume first that $A \bigwedge_{\Gamma}{ }^{p} H^{\cdot}\left(i_{w^{\prime}} i_{w^{\prime}}^{*} K\right)$ for any $w^{\prime} \in W$ such that $w^{\prime}<w$. Then $A \bigwedge_{\Gamma}{ }^{p} H^{\cdot}\left(u_{!} u^{*} K\right)$ where $u: \cup_{w^{\prime} ; w^{\prime}<w} \mathcal{B}_{w^{\prime}} \rightarrow \mathcal{B}$ is the inclusion. Let $u^{\prime}: \cup_{w^{\prime} ; w^{\prime} \leq w} \mathcal{B}_{w^{\prime}} \rightarrow \mathcal{B}$ be the inclusion. Since $i_{w !} i_{w}^{*} K=A^{\prime}$ we see that $A \dashv_{\Gamma}{ }^{p} H^{*}\left(u_{!}^{\prime} u^{\prime *} K\right)$. Thus $A \dashv_{\Gamma} K$ hence $A \cong K$ and $A \in \mathbb{S}(\mathcal{B})$.

Next we assume that $A \dashv_{\Gamma}{ }^{p} H^{\cdot}\left(i_{w^{\prime} !} i_{w^{\prime}}^{*} K\right)$ for some $w^{\prime} \in W$ such that $w^{\prime}<$ $w$. It follows that there exists $j^{\prime}$ such that $\left.A \dashv_{\Gamma}{ }^{p} H^{\cdot}\left(i_{w^{\prime} !}{ }^{p} H^{j^{\prime}}\left(i_{w^{\prime}}^{*} K\right)\right)\right)$. Hence there exists a simple object $A^{\prime \prime}$ of $\mathcal{M}_{\Gamma}\left(\mathcal{B}_{w^{\prime}}\right)$ such that $A^{\prime \prime} \dashv^{p} H^{j^{\prime}}\left(i_{w^{\prime}}^{*} K\right)$ and $A \dashv_{\Gamma}$ ${ }^{p} H^{\cdot}\left(i_{w^{\prime} !} A^{\prime \prime}\right)$. From 4.14 we see that $A^{\prime \prime} \in \mathbb{S}\left(\mathcal{B}_{w^{\prime}}\right)$. From the induction hypothesis we see that $A \in \mathbb{S}(\mathcal{B})$. This proves (a).

7.4. Let $w \in W$. Let $f_{w}: \tilde{\mathcal{B}}_{w} \rightarrow \mathcal{B}_{w}$ be as in 4.7, a finite principal covering. Let $U_{w}=\left\{u \in U ; \dot{w} F(u) \dot{w}^{-1} \in U\right\}$. Let $\hat{\mathcal{B}}_{w}=\left\{z \in G ; z^{-1} F(z) \in U \dot{w}\right\}$. Define $a^{\prime}: \hat{\mathcal{B}}_{w} \rightarrow \tilde{\mathcal{B}}_{w}$ by $z \mapsto z U$, a principal $U_{w}$-bundle. Let

$$
\begin{aligned}
& X_{w}=\left\{\left(B^{\prime}, g\right) \in \mathcal{B} \times G ; g^{-1} F(g) \in U_{B^{\prime}}, \operatorname{pos}\left(B^{\prime}, F\left(B^{\prime}\right)\right)=w\right\}, \\
& \tilde{X}_{w}=\left\{\left(z U_{w}, g\right) \in G / U_{w} \times G ; z^{-1} F(z) \in U \dot{w}, g^{-1} F(g) \in z U z^{-1}\right\}, \\
& \tilde{X}_{w}^{\prime}=\left\{(z, y) \in G \times G ; z^{-1} F(z) \in U \dot{w}, y^{-1} F(y) \in U \dot{w}\right\}, \\
& \tilde{X}_{w}^{\prime \prime}=U_{w} \backslash \tilde{X}_{w}^{\prime},
\end{aligned}
$$

where $U_{w}$ acts (freely) by $u:(z, y) \mapsto\left(z u^{-1}, y u^{-1}\right)$. Define $\pi_{w}: X_{w} \rightarrow \mathcal{B}_{w}$ by $\left(B^{\prime}, g\right) \mapsto B^{\prime}$. Define $\pi^{\prime}: \tilde{X}_{w} \rightarrow \tilde{\mathcal{B}}_{w}$ by $\left(z U_{w}, g\right) \mapsto z U$. Under the identification $\tilde{X}_{w}=\tilde{X}_{w}^{\prime \prime},\left(z U_{w}, g\right) \leftrightarrow(z, g z) U_{w}, \pi^{\prime}$ becomes $\pi^{\prime \prime}: \tilde{X}_{w}^{\prime \prime} \rightarrow \tilde{\mathcal{B}}_{w},(z, y) U_{w} \mapsto z U$. Define $a: \tilde{X}_{w}^{\prime} \rightarrow \tilde{X}_{w}^{\prime \prime}$ by $(z, y) \mapsto(z, y) U_{w}$. Define $h: \tilde{X}_{w}^{\prime} \rightarrow \hat{\mathcal{B}}_{w}$ by $(z, y) \mapsto z$. Define $\gamma: \tilde{X}_{w} \rightarrow X_{w}$ by $\left(z U_{w}, g\right) \mapsto\left(z B z^{-1}, g\right)$. Now $\Gamma$ acts on $\tilde{X}_{w}^{\prime \prime}$ by $g_{0}:(z, y) \mapsto$ $\left(g_{0} z, y\right)$. We show:

(i) if $A \in \mathcal{M}_{\Gamma}\left(\tilde{\mathcal{B}}_{w}\right)$ is simple and $A \dashv_{\Gamma}{ }^{p} H^{\cdot}\left(h_{!} \overline{\mathbf{Q}}_{l}\right)$, then $A \cong \overline{\mathbf{Q}}_{l}[\operatorname{dim} U]$;

(ii) if $A \in \mathcal{M}_{\Gamma}\left(\tilde{\mathcal{B}}_{w}\right)$ is simple and $A \dashv_{\Gamma}{ }^{p} H^{\cdot}\left(a_{l}^{\prime} h_{l} \overline{\mathbf{Q}}_{l}\right)$, then $A \cong \overline{\mathbf{Q}}_{l}[l(w)]$;

(iii) if $A \in \mathcal{M}_{\Gamma}\left(\tilde{\mathcal{B}}_{w}\right)$ is simple and $A \dashv_{\Gamma}{ }^{p} H^{\cdot}\left(\pi_{!}^{\prime \prime} a_{!} \overline{\mathbf{Q}}_{l}\right)$, then $A \cong \overline{\mathbf{Q}}_{l}[l(w)]$;

(iv) if $A \in \mathcal{M}_{\Gamma}\left(\tilde{\mathcal{B}}_{w}\right)$ is simple and $A \dashv_{\Gamma}{ }^{p} H^{\cdot}\left(\pi_{!}^{\prime} \overline{\mathbf{Q}}_{l}\right)$, then $A \cong \overline{\mathbf{Q}}_{l}[l(w)]$;

(v) if $A \in \mathcal{M}_{\Gamma}\left(\mathcal{B}_{w}\right)$ is simple and $\left.A \dashv_{\Gamma}{ }^{p} H^{\cdot}\left(\pi_{w !} \overline{\mathbf{Q}}_{l}\right)\right)$, then $A \in \mathbb{S}\left(\mathcal{B}_{w}\right)$;

(vi) if $A \in \mathcal{M}_{\Gamma}(\mathcal{B})$ is simple and $A \dashv_{\Gamma}{ }^{p} H^{\cdot}\left(i_{w !} \pi_{w !} \overline{\mathbf{Q}}_{l}\right)$, then $A \in \mathbb{S}(\mathcal{B})$.

Now (i) is obvious; (ii) follows from (i) using $a_{1}^{\prime} \overline{\mathbf{Q}}_{l}=\overline{\mathbf{Q}}_{l}[-2 \delta](-\delta)$ where $\delta=$ $\operatorname{dim} U_{w}$; (iii) follows from (ii) using $a^{\prime} h=\pi^{\prime \prime} a$; (iv) follows from (iii) using $a_{!} \overline{\mathbf{Q}}_{l}=$ $\overline{\mathbf{Q}}_{l}[-2 \delta](-\delta)$ and the identification $\pi^{\prime}=\pi^{\prime \prime}$. Since the diagram formed by $\pi^{\prime}, f_{w}, \gamma, \pi_{w}$ is cartesian we have $\pi_{!}^{\prime} \overline{\mathbf{Q}}_{l}=\pi_{!}^{\prime} \gamma^{*} \overline{\mathbf{Q}}_{l}=f_{w}^{*} \pi_{w !} \overline{\mathbf{Q}}_{l}$.

We prove (v). Let $A$ be as in (v). Let $A^{\prime}$ be a simple object of $\mathcal{M}_{\Gamma}\left(\tilde{\mathcal{B}}_{w}\right)$ such that $A^{\prime} \dashv_{\Gamma} f_{w}^{*} A$. We have

$$
A^{\prime} \dashv_{\Gamma} f_{w}^{*}\left({ }^{p} H^{\cdot}\left(\pi_{w !} \overline{\mathbf{Q}}_{l}\right)\right)={ }^{p} H^{\cdot}\left(f_{w}^{*} \pi_{w !} \overline{\mathbf{Q}}_{l}\right)={ }^{p} H^{\cdot}\left(\pi_{!}^{\prime} \overline{\mathbf{Q}}_{l}\right)
$$

and using (iv) we see that $A^{\prime} \cong \overline{\mathbf{Q}}_{l}[l(w)]$. Then there exists a nonzero morphism $f_{w}^{*} A \rightarrow \overline{\mathbf{Q}}_{l}[l(w)]$ in $\mathcal{M}_{\Gamma}\left(\tilde{\mathcal{B}}_{w}\right)$. Hence there exists a nonzero morphism $A \rightarrow f_{w !} \overline{\mathbf{Q}}_{l}[l(w)]$ in $\mathcal{M}_{\Gamma}\left(\mathcal{B}_{w}\right)$. Now $f_{w !} \overline{\mathbf{Q}}_{l}[l(w)]=\oplus_{\mathcal{L}} \mathcal{L}_{w}[l(w)]$ where $\mathcal{L}$ runs over the local systems in $\mathcal{S}(T)^{w F}$ (up to isomorphism). We must have $A \cong \mathcal{L}_{w}[l(w)]$ for some $\mathcal{L}$ as above. This proves (v). 
We prove (vi). We have $A \dashv_{\Gamma}{ }^{p} H \cdot\left(i_{w !}\left({ }^{p} H^{j^{\prime}}\left(\pi_{w !} \overline{\mathbf{Q}}_{l}\right)\right)\right)$ for some $j^{\prime}$. Hence there exists a simple object $A_{1}$ of $\mathcal{M}_{\Gamma}\left(\mathcal{B}_{w}\right)$ such that $A_{1} \dashv_{\Gamma}{ }^{p} H^{j^{\prime}}\left(\pi_{w} ! \overline{\mathbf{Q}}_{l}\right)$ and $A \dashv_{\Gamma}$ ${ }^{p} H^{\cdot}\left(i_{w} A_{1}\right)$. From (v) we see that $A_{1} \in \mathbb{S}\left(\mathcal{B}_{w}\right)$. From 7.3 we see that $A \in \mathbb{S}(\mathcal{B})$.

7.5. Let $J \subset \mathbf{I}$. Define $\pi_{J}: X \rightarrow \mathcal{P}_{J}$ by $\left(B^{\prime}, g\right) \mapsto P_{B^{\prime}, J}$. This is compatible with the $\Gamma$-actions where $\Gamma$ acts on $X$ by $g_{0}:\left(B^{\prime}, g\right) \mapsto\left(g_{0} B^{\prime} g_{0}^{-1}, g g_{0}^{-1}\right)$. Hence ${ }^{p} H^{j}\left(\pi_{J !} \overline{\mathbf{Q}}_{l}\right)$ has a $\Gamma$-equivariant structure. We show:

Theorem 7.6. (a) If $A$ is a simple object of $\mathcal{M}_{\Gamma}\left(\mathcal{P}_{J}\right)$ such that $A \dashv_{\Gamma}{ }^{p} H^{\cdot}\left(\pi_{J !} \overline{\mathbf{Q}}_{l}\right)$, then $A \in \mathbb{S}\left(\mathcal{P}_{J}\right)$.

Note that $\pi_{J}$ is a composition $\pi^{\prime} \pi^{\prime \prime}$ where $\pi^{\prime \prime}: X \rightarrow \mathcal{B}$ is $\left(B^{\prime}, g\right) \mapsto B^{\prime}$ and $\pi^{\prime \prime}: \mathcal{B} \rightarrow \mathcal{P}_{J}$ is $B^{\prime} \mapsto P_{B^{\prime}, J}$. We have a spectral sequence in $\mathcal{M}_{\Gamma}\left(\mathcal{P}_{J}\right)$ with $E_{2}={ }^{p} H^{\cdot}\left(\pi_{!}^{\prime}\left({ }^{p} H^{\cdot}\left(\pi_{!}^{\prime \prime} \overline{\mathbf{Q}}_{l}\right)\right)\right)$ and $E_{\infty}$ is an associated graded of ${ }^{p} H^{\cdot}\left(\pi_{!}^{\prime} \pi_{!}^{\prime \prime} \overline{\mathbf{Q}}_{l}\right)$. We have $A \dashv_{\Gamma} E_{\infty}$ hence $A \dashv_{\Gamma} E_{2}$. Hence we can find a simple object $A_{1} \in \mathcal{M}_{\Gamma}(\mathcal{B})$ such that $A_{1} \dashv_{\Gamma}{ }^{p} H^{\cdot}\left(\pi_{!}^{\prime \prime} \overline{\mathbf{Q}}_{l}\right)$ and $A \dashv_{\Gamma}{ }^{p} H^{\cdot}\left(\pi_{!}^{\prime} A_{1}\right)$. Using the partition $\mathcal{B}=\sqcup_{w} \mathcal{B}_{w}$, we see that $A_{1} \dashv_{\Gamma}{ }^{p} H^{\cdot}\left(i_{w} i_{w}^{*} \pi_{!}^{\prime \prime} \overline{\mathbf{Q}}_{l}\right)$ for some $w \in W$ (with $i_{w}$ as in 7.3). Since $i_{w}^{*} \pi_{!}^{\prime \prime} \overline{\mathbf{Q}}_{l}=$ $\pi_{w !} \overline{\mathbf{Q}}_{l}$ (with $\pi_{w}$ as in 7.4), we see that $A_{1} \dashv_{\Gamma}{ }^{p} H^{\cdot}\left(i_{w} ! \pi_{w !} \overline{\mathbf{Q}}_{l}\right)$. Using 7.4 (vi) we see that $A_{1} \in \mathbb{S}\left(\mathcal{B}_{w}\right)$. By 4.13 (for $\mathcal{B}$ instead of $\mathcal{P}_{J}$ ) we see that $A_{1} \dashv_{\Gamma}{ }^{p} H \cdot\left({ }^{\emptyset} \bar{\Upsilon}_{!}^{\mathbf{s}} \overline{\mathcal{L}}^{\sharp}\right.$ ) for some sequence $\mathbf{s}$ in $\mathbf{I}$ and some $\mathcal{L} \in \mathcal{S}(T)^{[\mathbf{s}] F}$ (here ${ }^{\emptyset} \bar{\Upsilon}^{\mathbf{s}}$ is $\bar{\Upsilon}^{\mathbf{s}}$ of 3.2 with $J$ replaced by $\emptyset)$. We have a spectral sequence in $\mathcal{M}_{\Gamma}\left(\mathcal{P}_{J}\right)$ with $E_{2}={ }^{p} H^{\cdot}\left(\pi_{!}^{\prime}\left({ }^{p} H^{\cdot}\left({ }^{\emptyset} \bar{\Upsilon}_{!}^{\mathbf{s}} \overline{\mathcal{L}}^{\sharp}\right)\right)\right)$ and $E_{\infty}$ is an associated graded of

$$
{ }^{p} H^{\cdot}\left(\pi_{!}^{\prime \emptyset} \bar{\Upsilon}_{!}^{\mathbf{s}} \overline{\mathcal{L}}^{\sharp}\right)={ }^{p} H^{\cdot}\left(\bar{\Upsilon}_{!}^{\mathbf{s}} \overline{\mathcal{L}}^{\sharp}\right)
$$

(with $\bar{\Upsilon}^{\mathbf{s}}$ as in 3.2). Now $A_{1}$ is a direct summand of ${ }^{p} H^{\cdot}\left({ }^{\emptyset} \bar{\Upsilon}_{!}^{\mathbf{s}} \overline{\mathcal{L}}^{\sharp}\right)$. Hence ${ }^{p} H^{\cdot}\left(\pi_{!}^{\prime} A_{1}\right)$ is a direct summand of ${ }^{p} H^{\cdot}\left(\pi_{!}^{\prime}\left({ }^{p} H^{\cdot}\left({ }^{\emptyset} \bar{\Upsilon}_{!}^{s} \overline{\mathcal{L}}^{\sharp}\right)\right)\right)$. Hence $A \dashv_{\Gamma} E_{2}$. Our spectral sequence is degenerate (by an argument as in 4.8). Hence $A \dashv_{\Gamma} E_{\infty}$ and $A \dashv_{\Gamma}$ ${ }^{p} H^{\cdot}\left(\bar{\Upsilon}_{!}^{\mathrm{s}} \overline{\mathcal{L}}^{\sharp}\right)$. Using 4.13 we deduce that $A \in \mathbb{S}\left(\mathcal{P}_{J}\right)$. The theorem is proved.

7.7. We have a $\Gamma$-action $g_{1}:\left(B^{\prime}, g\right) \mapsto\left(B^{\prime}, g_{1} g\right)$ on $X$ (this is different from the $\Gamma$-action on $X$ in 7.5). This induces a $\Gamma$-module structure on $H_{c}^{2 d}\left(X, \overline{\mathbf{Q}}_{l}\right)(d$ as in 7.1).

Proposition 7.8. The $\Gamma$-module $H_{c}^{2 d}\left(X, \overline{\mathbf{Q}}_{l}\right)$ contains a copy of Reg, the left regular representation of $\Gamma$.

Let $\rho_{1}: X \rightarrow X^{\prime}$ be as in 7.1. Let $X^{\prime \prime}=\{u \in G ; u$ unipotent $\}$. Define $\rho_{2}: X^{\prime} \rightarrow X^{\prime \prime}$ by $\left(B^{\prime}, u\right) \mapsto u$. Let $\rho=\rho_{2} \rho_{1}: X \rightarrow X^{\prime \prime}$. It is well known that $\rho_{2}$ is a semismall morphism. Recall that $\rho_{1}$ is a finite étale covering. Hence $\rho$ is a semismall morphism. Using this, we see that $\rho_{!} \overline{\mathbf{Q}}_{l}[2 d]$ is a perverse sheaf on $X^{\prime}$ (recall that $X$ is smooth of pure dimension $2 d$ and $\rho$ is proper). By the decomposition theorem $\left[\mathrm{BBD},{ }^{p} H^{\cdot}\left(\rho_{!} \overline{\mathbf{Q}}_{l}\right)\right.$ is a semisimple perverse sheaf. Hence $\rho_{!} \overline{\mathbf{Q}}_{l}[2 d]$ is a semisimple perverse sheaf. Now $\Gamma$ acts on $X^{\prime \prime}$ trivially and $\rho$ is compatible with the $\Gamma$-actions. Since $\overline{\mathbf{Q}}_{l}$ is naturally a $\Gamma$-equivariant local system on $X$ we see that we have naturally $\rho_{!} \overline{\mathbf{Q}}_{l}[2 d] \in \mathcal{M}_{\Gamma}\left(X^{\prime \prime}\right)$. Let $j:\{1\} \rightarrow X^{\prime \prime}$ be the inclusion of the unit element into $X^{\prime \prime}$. If $E$ is an irreducible $\Gamma$-module over $\overline{\mathbf{Q}}_{l}$ we can regard $E$ as a $\Gamma$-equivariant local system on $\{1\}$. Then $j_{!} E$ is a simple object of $\mathcal{M}_{\Gamma}\left(X^{\prime \prime}\right)$. Let $n_{E}$ be the number of times $j_{!} E$ appears in a direct sum decomposition of $\rho_{!} \overline{\mathbf{Q}}_{l}[2 d]$ (a semisimple object of $\mathcal{M}_{\Gamma}\left(X^{\prime \prime}\right)$ ) into a direct sum of simple objects. Since $\rho_{!} \overline{\mathbf{Q}}_{l}[2 d]$ 
is selfdual, we have using 1.6(a):

$$
\begin{aligned}
& n_{E}=\operatorname{dim} H_{c}^{0}\left(X^{\prime \prime}, j_{!} E \otimes \rho_{!} \overline{\mathbf{Q}}_{l}[2 d]\right)^{\Gamma}=\operatorname{dim} H_{c}^{2 d}\left(\{1\}, E \otimes j^{*} \rho_{!} \overline{\mathbf{Q}}_{l}\right)^{\Gamma} \\
& =\operatorname{dim}\left(H_{c}^{2 d}\left(\mathcal{B} \times \Gamma, \overline{\mathbf{Q}}_{l}\right) \otimes E\right)^{\Gamma}=\operatorname{dim}(\operatorname{Reg}(-d) \otimes E)^{\Gamma}=\operatorname{dim} E
\end{aligned}
$$

We see that $\rho_{!} \overline{\mathbf{Q}}_{l}[2 d]$ contains as a direct summand the perverse sheaf $j_{!}$Reg where $R e g$ is regarded as an object of $\mathcal{M}_{\Gamma}(\{1\})$. It follows that the $\Gamma$-module $H_{c}^{0}\left(\rho_{!} \overline{\mathbf{Q}}_{l}[2 d]\right)$ contains as a direct summand the $\Gamma$-module $H_{c}^{0}\left(j_{!} \operatorname{Reg}\right)=$ Reg. Equivalently the $\Gamma$ module $H^{2 d}\left(X, \overline{\mathbf{Q}}_{l}\right)$ contains Reg as a direct summand. The proposition is proved.

Corollary 7.9. Let $E$ be an irreducible $\Gamma$-module over $\overline{\mathbf{Q}}_{l}$. There exists $w \in W$ such that $E$ appears in the $\Gamma$-module $\oplus_{i} H_{c}^{i}\left(\tilde{\mathcal{B}}_{w}, \overline{\mathbf{Q}}_{l}\right)$. ( $\Gamma$ acts on $\tilde{\mathcal{B}}_{w}$ by $g_{1}: z U \rightarrow g_{1} z U$.)

From 7.8 we see that $E$ appears in the $\Gamma$-module $H_{c}^{2 d}\left(X, \overline{\mathbf{Q}}_{l}\right)$. Using this and the partition $X=\sqcup_{w \in W} X_{w}$ (with $X_{w}$ as in 7.4, $\Gamma$-stable) we see that there exists $w \in W$ such that $E$ appears in the $\Gamma$-module $H_{c}^{2 d}\left(X_{w}, \overline{\mathbf{Q}}_{l}\right)$.

Now $\Gamma$ acts:

on $\tilde{X}_{w}$ by $g_{1}:\left(z U_{w}, g\right) \mapsto\left(z U_{w}, g_{1} g\right)$,

on $\tilde{X}_{w}^{\prime}$ by $g_{1}:(z, y) \mapsto\left(z, g_{1} y\right)$,

on $\tilde{X}_{w}^{\prime \prime}$ by $g_{1}:(z, y) U_{w} \mapsto\left(z, g_{1} y\right) U_{w}$,

on $\hat{\mathcal{B}}_{w}$ by $g_{1}: z \mapsto g_{1} z$.

(Notation of 7.4.) Moreover, the maps $\gamma, a, a^{\prime}$ in 7.4 are compatible with the $\Gamma$-actions. Since $\gamma$ is a finite principal covering we see that $E$ appears in the $\Gamma$ module $H_{c}^{2 d}\left(\tilde{X}_{w}, \overline{\mathbf{Q}}_{l}\right)$. We identify $\tilde{X}_{w}=\tilde{X}_{w}^{\prime \prime}$ as in 7.4. We see that $E$ appears in the $\Gamma$-module $H_{c}^{2 d}\left(\tilde{X}_{w}^{\prime \prime}, \overline{\mathbf{Q}}_{l}\right)$. Since $a$ is an affine space bundle we see that $E$ appears in the $\Gamma$-module $H_{c}^{4 d-2 l(w)}\left(\tilde{X}_{w}^{\prime}, \overline{\mathbf{Q}}_{l}\right)$. We have $\tilde{X}_{w}^{\prime}=\hat{\mathcal{B}}_{w} \times \hat{\mathcal{B}}_{w}$ and $\oplus_{i} H_{c}^{i}\left(\tilde{X}_{w}^{\prime}, \overline{\mathbf{Q}}_{l}\right)=$ $\oplus_{i, i^{\prime}} H_{c}^{i}\left(\hat{\mathcal{B}}_{w}, \overline{\mathbf{Q}}_{l}\right) \otimes H_{c}^{i^{\prime}}\left(\hat{\mathcal{B}}_{w}, \overline{\mathbf{Q}}_{l}\right)$ with $\Gamma$ acting only on the seond factor. It follows that $E$ appears in the $\Gamma$-module $\oplus_{i} H_{c}^{i}\left(\hat{\mathcal{B}}_{w}, \overline{\mathbf{Q}}_{l}\right)$. Since $a^{\prime}$ is an affine space bundle we see that the corollary holds.

7.10. For $w \in W$ let $\mathfrak{T}_{w}=\left\{t \in T ; \dot{w} F(t) \dot{w}^{-1}=t\right\}$. Now $\Gamma \times \Gamma \times \mathfrak{T}_{w}$ acts on $X_{w}$ by $\left(g_{0}, g_{1}, t\right):\left(B^{\prime}, g\right) \mapsto\left(g_{0} B^{\prime} g_{0}^{-1}, g_{1} g g_{0}^{-1}\right)$, on $\tilde{X}_{w}$ by $\left(g_{0}, g_{1}, t\right):\left(z U_{w}, g\right) \mapsto$ $\left(g_{0} z t^{-1} U_{w}, g_{1} g g_{0}^{-1}\right)$, on $\tilde{X}_{w}^{\prime}$ by $\left(g_{0}, g_{1}, t\right):(z, y) \mapsto\left(g_{0} z t^{-1}, g_{1} y t^{-1}\right)$, on $\tilde{X}_{w}^{\prime \prime}$ by $\left(g_{0}, g_{1}, t\right):(z, y) U_{w} \mapsto\left(g_{0} z t^{-1}, g_{1} y t^{-1}\right) U_{w}$. Moreover, the maps $\gamma, a$ in 7.4 are compatible with the $\Gamma \times \Gamma \times \mathfrak{T}_{w}$-actions. Also $\Gamma \times \mathfrak{T}_{w}$ acts on $\hat{\mathcal{B}}_{w}$ by $\left(g_{1}, t\right): z \mapsto$ $g_{1} z t^{-1}$, on $\tilde{\mathcal{B}}_{w}$ by $\left(g_{1}, t\right): z U \mapsto g_{1} z t^{-1} U$; the map $a^{\prime}$ in 7.4 is compatible with the $\Gamma \times \mathfrak{T}_{w}$-actions.

For any $\theta \in \hat{\mathfrak{T}}_{w}:=\operatorname{Hom}\left(\mathfrak{T}_{w}, \overline{\mathbf{Q}}_{l}^{*}\right)$ let $H_{c}^{i}\left(\tilde{\mathcal{B}}_{w}\right)_{\theta}$ be the subspace of $H_{c}^{i}\left(\tilde{\mathcal{B}}_{w}\right)$ on which $\mathfrak{T}_{w}$ acts via $\theta$; this is naturally a $\Gamma \times \mathfrak{T}_{w}$-module. Now $\Gamma \times \Gamma$ acts on $X$ by $\left(g_{0}, g_{1}\right):\left(B^{\prime}, g\right) \mapsto\left(g_{0} B^{\prime} g_{0}^{-1}, g_{1} g g_{0}^{-1}\right)$.

Let $\mathcal{G}(\Gamma)$ (resp. $\mathcal{G}(\Gamma \times \Gamma)$ or $\left.\mathcal{G}\left(\Gamma \times \Gamma \times \mathfrak{T}_{w}\right)\right)$ be the Grothendieck group of $\Gamma$-modules (resp $\Gamma \times \Gamma$-modules or $\Gamma \times \Gamma \times \mathfrak{T}_{w}$-modules) of finite dimension over $\overline{\mathbf{Q}}_{l}$. Let $\Pi: \mathcal{G}\left(\Gamma \times \Gamma \times \mathfrak{T}_{w}\right) \rightarrow \mathcal{G}\left(\Gamma \times \Gamma \times \mathfrak{T}_{w}\right)$ be the homomorphism which takes an irreducible $\Gamma \times \Gamma \times \mathfrak{T}_{w}$-module to the space of $\mathfrak{T}_{w}$-invariants (an irreducible $\Gamma \times \Gamma \times \mathfrak{T}_{w}$-module or 0$)$. In the setup of 7.9 we show:

\section{Proposition 7.11.}

(a) $\quad \sum_{i}(-1)^{i} H_{c}^{i}\left(X, \overline{\mathbf{Q}}_{l}\right)=\sum_{\substack{w \in W \\ \theta \in \tilde{\mathcal{T}}^{\prime} \\ i, i^{\prime}}}(-1)^{i+i^{\prime}} H_{c}^{i}\left(\tilde{\mathcal{B}}_{w}, \overline{\mathbf{Q}}_{l}\right)_{\theta^{-1}} \otimes H_{c}^{i^{\prime}}\left(\tilde{\mathcal{B}}_{w}, \overline{\mathbf{Q}}_{l}\right)_{\theta}$. 
equality in $\mathcal{G}(\Gamma \times \Gamma)$.

By the arguments in the proof of 7.9 we have

$$
\sum_{i}(-1)^{i} H_{c}^{i}\left(X, \overline{\mathbf{Q}}_{l}\right)=\sum_{w \in W} \sum_{i}(-1)^{i} H_{c}^{i}\left(X_{w}, \overline{\mathbf{Q}}_{l}\right),
$$

equality in $\mathcal{G}(\Gamma \times \Gamma)$ and

$$
\begin{aligned}
& \sum_{i}(-1)^{i} H_{c}^{i}\left(X_{w}, \overline{\mathbf{Q}}_{l}\right)=\Pi\left(\sum_{i}(-1)^{i} H_{c}^{i}\left(\tilde{X}_{w}, \overline{\mathbf{Q}}_{l}\right)\right)=\Pi\left(\sum_{i}(-1)^{i} H_{c}^{i}\left(\tilde{X}_{w}^{\prime \prime}, \overline{\mathbf{Q}}_{l}\right)\right) \\
& =\Pi\left(\sum_{i}(-1)^{i} H_{c}^{i}\left(\tilde{X}_{w}^{\prime}, \overline{\mathbf{Q}}_{l}\right)\right)=\Pi\left(\sum_{i, i^{\prime}}(-1)^{i+i^{\prime}} H_{c}^{i}\left(\hat{\mathcal{B}}_{w}, \overline{\mathbf{Q}}_{l}\right) \otimes H_{c}^{i^{\prime}}\left(\hat{\mathcal{B}}_{w}, \overline{\mathbf{Q}}_{l}\right)\right) \\
& =\sum_{\theta \in \hat{\mathfrak{Z}}_{w}} \sum_{i, i^{\prime}}(-1)^{i+i^{\prime}} H_{c}^{i}\left(\hat{\mathcal{B}}_{w}, \overline{\mathbf{Q}}_{l}\right)_{\theta-1} \otimes H_{c}^{i^{\prime}}\left(\hat{\mathcal{B}}_{w}, \overline{\mathbf{Q}}_{l}\right)_{\theta} \\
& =\sum_{\theta \in \hat{\mathfrak{T}}_{w}} \sum_{i, i^{\prime}}(-1)^{i+i^{\prime}} H_{c}^{i}\left(\tilde{\mathcal{B}}_{w}, \overline{\mathbf{Q}}_{l}\right)_{\theta-1} \otimes H_{c}^{i^{\prime}}\left(\tilde{\mathcal{B}}_{w}, \overline{\mathbf{Q}}_{l}\right)_{\theta},
\end{aligned}
$$

equalities in $\mathcal{G}\left(\Gamma \times \Gamma \times \mathfrak{T}_{w}\right)$. This proves (a).

7.12. Restricting the $\Gamma \times \Gamma$-action on the modules in 7.11 (a) to $\Gamma$ by $g_{1} \mapsto\left(1, g_{1}\right)$ gives

$$
\sum_{i}(-1)^{i} H_{c}^{i}\left(X, \overline{\mathbf{Q}}_{l}\right)=\sum_{\substack{w \in W \\ \theta \in \tilde{\mathcal{F}}^{\prime} \\ i, i^{\prime}}}(-1)^{i+i^{\prime}} \operatorname{dim}\left(H_{c}^{i}\left(\tilde{\mathcal{B}}_{w}, \overline{\mathbf{Q}}_{l}\right)_{\theta^{-1}}\right) H_{c}^{i^{\prime}}\left(\tilde{\mathcal{B}}_{w}, \overline{\mathbf{Q}}_{l}\right)_{\theta} .
$$

equality in $\mathcal{G}(\Gamma)$. From [DL $7.1,(7.6 .3)]$ we see that the right hand side is equal to $|W|$ Reg. Thus we have

\section{Proposition 7.13.}

$$
\sum_{i}(-1)^{i} H_{c}^{i}\left(X, \overline{\mathbf{Q}}_{l}\right)=|W| \operatorname{Reg} \text { in } \mathcal{G}(\Gamma) .
$$

Lemma 7.14. Assume that $G$ is simply connected. Let $\mathbf{s}=\left(s_{1}, \ldots, s_{r}\right), \mathcal{L}$ be as in 2.6 and that 6.18(a) holds. Let $Z^{\mathbf{s}}$ be as in 2.5. Let $\dot{Z}$ be as in 2.5 (with $\mathbf{w}=\mathbf{s}$ ). Let $Z=\mathcal{Z}^{\mathbf{s}}$ be as in 2.6. Then:

(a) $\operatorname{dim} H_{c}^{2 r}(Z, \overline{\mathcal{L}})=n_{\mathcal{L}}$

(b) $\operatorname{dim} H_{c}^{2 r}\left(Z^{\mathbf{s}}, \mathcal{L}_{\mathbf{s}}\right)=n_{\mathcal{L}}$;

(c) $\operatorname{dim} H_{c}^{2 r}\left(\dot{Z}, \overline{\mathbf{Q}}_{l}\right)=1$.

We prove (a). We shall use 6.18(b) assuming that $\tilde{\mathbf{s}}=\mathbf{s}$. Then $Z=Z^{\prime}$ and $\operatorname{dim} Z=r$ hence $H_{c}^{i}(Z, \overline{\mathcal{L}})=0$ for $i<2 r$ and $H_{c}^{i}(Z, \overline{\tilde{\mathcal{L}}})=0$ for $i<2 r$. Hence $H_{c}^{4 r}(Z \times Z, \overline{\mathcal{L}} \otimes \overline{\tilde{\mathcal{L}}})=H_{c}^{2 r}(Z, \overline{\mathcal{L}}) \otimes H_{c}^{2 r}(Z, \overline{\tilde{\mathcal{L}}})$. Since in this case $\rho=2 r$ we see from 6.18 (b) that $\operatorname{dim}\left(H_{c}^{2 r}(Z, \overline{\mathcal{L}}) \otimes H_{c}^{2 r}(Z, \overline{\tilde{\mathcal{L}}})\right)^{\Gamma}=n_{\mathcal{L}}$. Using Poincaré duality on the smooth variety $Z$ of pure dimension $r$ (see 2.9) we deduce that $\operatorname{dim}\left(H^{0}(Z, \overline{\tilde{\mathcal{L}}}) \otimes\right.$ $\left.H^{0}(Z, \overline{\mathcal{L}})\right)^{\Gamma}=n_{\mathcal{L}}$. Note that the $\Gamma$-modules $H^{0}(Z, \overline{\tilde{\mathcal{L}}}), H^{0}(Z, \overline{\mathcal{L}})$ are dual to each other. Hence the previous equality can be written as $\operatorname{dim}_{\operatorname{End}_{\Gamma}}\left(H^{0}(Z, \overline{\mathcal{L}})\right)=n_{\mathcal{L}}$ where $\operatorname{End}_{\Gamma}()$ is the space of $\Gamma$-module endomorphisms. If $H^{0}(Z, \overline{\mathcal{L}})=0$, it follows that $n_{\lambda}=0$; in this case we have also $H^{0}(Z, \overline{\tilde{\mathcal{L}}})=0$ and by Poincaré duality, $H_{c}^{2 r}(Z, \overline{\mathcal{L}})=0$ and (a) holds. Thus we may assume that $H^{0}(Z, \overline{\mathcal{L}}) \neq 0$. Then $\operatorname{dim} \operatorname{End}_{\Gamma}\left(H^{0}(Z, \overline{\mathcal{L}})\right) \geq 1$ hence $\operatorname{dim}_{\operatorname{End}_{\Gamma}}\left(H^{0}(Z, \overline{\mathcal{L}})\right)=n_{\mathcal{L}}=1$. Then $R=R_{\mathcal{L}}$. 
Since $G$ is simply connected, it follows that $\mathcal{L} \cong \overline{\mathbf{Q}}_{l}$. Then $H^{0}(Z, \overline{\mathcal{L}})=H^{0}\left(Z, \overline{\mathbf{Q}}_{l}\right)$ may be identified with the permutation representation $V$ of $\Gamma$ on the set $C$ of connected components of $Z$. Let $\Gamma^{\prime}$ be the isotropy group in $\Gamma$ of some connected component of $Z$. Since $\Gamma$ acts transitively on $C$ (see 2.9 ) we see that $\operatorname{dim} V=\left|\Gamma / \Gamma^{\prime}\right|$, $1=\operatorname{dim} \operatorname{End}_{G}(V)=\left|\Gamma^{\prime} \backslash \Gamma / \Gamma^{\prime}\right|$. It follows that $\Gamma=\Gamma^{\prime}$ hence $\operatorname{dim} V=1$. Then we have $\operatorname{dim} H^{0}(Z, \overline{\tilde{\mathcal{L}}})=1$ and, by Poincaré duality, $\operatorname{dim} H_{c}^{2 r}(Z, \overline{\mathcal{L}})=1$. This proves (a).

We prove (b). Note that $Z^{\mathbf{s}}$ is an open dense subset of $Z$ (using the commutative diagram in 2.6 with $\mathcal{J}=\emptyset$ and 2.8, this statement is reduced to the statement that $Z_{2}^{\emptyset}$ in 2.6 is open dense in $Z_{2}$ which is clear). Note also that $\left.\overline{\mathcal{L}}\right|_{Z^{\mathrm{s}}}=\mathcal{L}_{\mathrm{s}}$ (see 2.12) and that $Z$ has pure dimension $r$ (see 2.9). We see that (b) follows from (a).

We prove (c). Let $f_{!}^{\chi} \overline{\mathbf{Q}}_{l}$ be as in 2.5 with $\mathbf{w}=\mathbf{s}$. From the definitions we have $H_{c}^{2 r}\left(\dot{Z}, \overline{\mathbf{Q}}_{l}\right)=\oplus_{\chi} H_{c}^{2 r}\left(Z^{\mathbf{s}}, f_{!}^{\chi} \overline{\mathbf{Q}}_{l}\right)$ where $\chi$ runs over $\operatorname{Hom}\left(T^{F^{\prime}}, \overline{\mathbf{Q}}_{l}^{*}\right)$ (as in 2.5). Using (b) and 2.5(a) we see that $\operatorname{dim} H_{c}^{2 r}\left(Z^{\mathbf{s}}, f_{!}^{\chi} \overline{\mathbf{Q}}_{l}\right)$ is 1 if $\chi=1$ and is 0 if $\chi \neq 1$. The result follows.

7.15. Assume that $G$ is simply connected. Let $d$ be as in 7.1. Let $w=w_{\mathbf{I}}$. We show:

(a) $\tilde{\mathcal{B}}_{w}$ is connected;

(b) $X_{w}$ is connected (notation of 7.4);

Assume that $\mathbf{s}$ is a reduced expression for $w$. The associated variety $\dot{Z}$ (see 2.5) is connected by $7.14(\mathrm{c})$ (note that $\dot{Z}$ has pure dimension $d=l(w)$ ). But $\dot{Z}$ may be identified with $\tilde{\mathcal{B}}_{w}$. Hence (a) holds. Since $a^{\prime}: \hat{\mathcal{B}}_{w} \rightarrow \tilde{\mathcal{B}}_{w}$ is a principal $U_{w}$-bundle (as in 7.4) we see that $\hat{\mathcal{B}}_{w}$ is connected. Since $\tilde{X}_{w}^{\prime} \cong \hat{\mathcal{B}}_{w} \times \hat{\mathcal{B}}_{w}$ (as in 7.4) we see that $\tilde{X}_{w}^{\prime}$ is connected. Since $a: \tilde{X}_{w}^{\prime} \rightarrow \tilde{X}_{w}^{\prime \prime}$ (as in 7.4) is a principal $U_{w}$-bundle we see that $\tilde{X}_{w}^{\prime \prime}$ is connected. Since $\tilde{X}_{w}=\tilde{X}_{w}^{\prime \prime}$ (as in 7.4) we see that $\tilde{X}_{w}$ is connected. Since $\gamma: \tilde{X}_{w} \rightarrow X_{w}$ (as in 7.4) is surjective, we see that $X_{w}$ is connected. Hence (b) holds.

Note that (a),(b) above do not necessarily hold without the assumption that $G$ is simply connected.

\subsection{6.}

Proof of Proposition 7.2. Note that $X=\cup_{w^{\prime} \in W} X_{w^{\prime}}$, that $X$ is of pure dimension $2 d$, that $X_{w}$ is an open subset of $X$ and that for any $w^{\prime} \in W-\{w\}, X_{w^{\prime}}$ has pure dimension equal to $d+l\left(w^{\prime}\right)<2 d$. It follows that $X$ is connected if and only if $X_{w}$ is connected. Hence 7.2 is a consequence of $7.15(\mathrm{~b})$.

\section{A COnjecture}

8.1. In this section we assume that $G$ has connected centre. Let $\mathcal{L} \in \mathcal{S}(T)$ be such that $(w F)^{*} \mathcal{L} \cong \mathcal{L}$ for some $w \in W$. Our assumption on $G$ guarantees that

(a) $w \in W_{\mathcal{L}}^{\prime} \Longrightarrow w \in W_{\mathcal{L}}$.

(Notation of 5.7.) Let $\mathcal{X}_{\mathcal{L}}$ be the set of all sequences $\mathbf{s}=\left(s_{1}, \ldots, s_{r}\right)$ in $\mathbf{I}$ such that $\mathcal{L} \in \mathcal{S}(T)^{[\mathbf{s}] F}$. Note that $\mathcal{X}_{\mathcal{L}} \neq \emptyset$.

To any $\mathbf{s} \in \mathcal{X}_{\mathcal{L}}$ we associate an element $\omega \in W$ as in 5.5. We show that $\omega$ is independent of the choice of $\mathbf{s}$. We must show that if $\tilde{\mathbf{s}}$ is another element of $\mathcal{X}_{\mathcal{L}}$ and $\tilde{\omega} \in W$ is associated to $\tilde{\mathbf{s}}$ in the same way as $\omega$ is associated to $\mathbf{s}$, then $\tilde{\omega}=\omega$. Using 5.6 we see that $F^{*} \omega^{*} \mathcal{L} \cong \mathcal{L}, F^{*} \tilde{\omega}^{*} \mathcal{L} \cong \mathcal{L}$. Hence $\omega^{*} \mathcal{L} \cong \tilde{\omega}^{*} \mathcal{L}$ so that $\tilde{\omega} \omega^{-1} \in W_{\mathcal{L}}^{\prime}$ and, using (a), $\tilde{\omega} \omega^{-1} \in W_{\mathcal{L}}$. By the proof of 5.8 (with $c=\mathbf{c}$ ) we 
have $\omega \mathbf{c}\left(R_{\mathcal{L}}^{+}\right)=R_{\mathcal{L}}^{+}$and similarly $\tilde{\omega} \mathbf{c}\left(R_{\mathcal{L}}^{+}\right)=R_{\mathcal{L}}^{+}$. Thus $\omega^{-1}\left(R_{\mathcal{L}}^{+}\right)=\tilde{\omega}^{-1}\left(R_{\mathcal{L}}^{+}\right)$and $\tilde{\omega} \omega^{-1}\left(R_{\mathcal{L}}^{+}\right)=R_{\mathcal{L}}^{+}$. This together with $\tilde{\omega} \omega^{-1} \in W_{\mathcal{L}}$ yields $\tilde{\omega}^{-1} \omega=1$, as desired.

Using the previous paragraph and (a) we see that for any $\mathbf{s}, \tilde{\mathbf{s}} \in \mathcal{X}_{\mathcal{L}}$, the set $\mathfrak{F}$ defined as in 5.9 (with $c=\mathbf{c}$ ) is equal to $\{1\}$.

8.2. For $\mathbf{s}, \tilde{\mathbf{s}} \in \mathcal{X}_{\mathcal{L}}$ let $Z, \tilde{Z}, r, \tilde{r}$ be as in 6.1 . Let $\overline{\mathcal{L}}$ be as in 6.2 and let $\overline{\tilde{\mathcal{L}}}$ be the analogous local system on $\tilde{Z}$ defined in terms of $\mathcal{L}$. Then $\overline{\tilde{\tilde{\mathcal{L}}}}$ (dual of $\overline{\tilde{\mathcal{L}}}$ ) is the analogous local system on $\tilde{Z}$ defined in terms of $\check{\mathcal{L}}$. Let

$$
V_{\tilde{\mathbf{s}}, \mathbf{s}}=\operatorname{Hom}_{\Gamma}\left(\oplus_{i} H_{c}^{i}(\tilde{Z}, \overline{\tilde{\mathcal{L}}})(i / 2), \oplus_{i^{\prime}} H_{c}^{i^{\prime}}(Z, \overline{\mathcal{L}})\left(i^{\prime} / 2\right)\right) .
$$

where $\operatorname{Hom}_{\Gamma}$ is the space of homomorphisms of $\Gamma$-modules. Using 2.13(a) we see that Poincaré duality holds on $\tilde{Z}$ in the form

$$
\operatorname{Hom}\left(H_{c}^{i}(\tilde{Z}, \overline{\tilde{\mathcal{L}}})(i / 2), \overline{\mathbf{Q}}_{l}\right)=H_{c}^{2 \tilde{r}-i}(\tilde{Z}, \tilde{\tilde{\tilde{\mathcal{L}}}})(\tilde{r}-i / 2) .
$$

Hence

$$
\begin{gathered}
\operatorname{Hom}\left(\oplus_{i} H_{c}^{i}(\tilde{Z}, \overline{\tilde{\mathcal{L}}})(i / 2), \overline{\mathbf{Q}}_{l}\right)=\oplus_{i} H_{c}^{i}(\tilde{Z}, \tilde{\tilde{\tilde{\mathcal{L}}}})(i / 2), \\
V_{\mathbf{s}, \tilde{\mathbf{s}}}=\left(\left(\oplus_{i^{\prime}} H_{c}^{i^{\prime}}(Z, \overline{\mathcal{L}})\left(i^{\prime} / 2\right)\right) \otimes\left(\oplus_{i} H_{c}^{i}(\tilde{Z}, \check{\tilde{\tilde{\mathcal{L}}}})(i / 2)\right)\right)^{\Gamma}, \\
V_{\mathbf{s}, \tilde{\mathbf{s}}}=\left(\oplus_{n} H_{c}^{n}(Z \times \tilde{Z}, \overline{\mathcal{L}} \otimes \overline{\tilde{\mathcal{L}}})(n / 2)\right)^{\Gamma} .
\end{gathered}
$$

By 6.14(d) and 6.15(b), the last vector space has a distinguished basis $\left\{b_{\mathbf{a}}^{1} ; \mathbf{a} \in \mathcal{A}_{1}\right\}$, with $\mathcal{A}_{1}$ as in 6.14 .

Let $C_{\mathcal{L}}$ be the category whose objects are the elements of $\mathcal{X}_{\mathcal{L}}$ and in which the set of morphisms from $\tilde{\mathbf{s}}$ to $\mathbf{s}$ is the vector space $V_{\tilde{\mathbf{s}}, \mathbf{s}}$. The composition of morphisms is given by composing linear maps.

8.3. We will view $T$ as a maximally $\mathbf{F}_{q}$-split torus of a second connected reductive algebraic group $G^{\prime}$ over $\mathbf{F}_{q}$ in such a way that $R_{\mathcal{L}}$ is the set of roots of $G^{\prime}$ with respect to $T^{\prime}$ and $R_{\mathcal{L}}^{+}$is the set of positive roots of $G^{\prime}$ with respect to $T^{\prime}$ and a Borel subgroup $B^{\prime}$ of $G^{\prime}$ which is defined over $\mathbf{F}_{q}$ and contains $T^{\prime}$.

Replacing $G, T, B, \mathcal{L}$ by $G^{\prime}, T, B^{\prime}, \overline{\mathbf{Q}}_{l}$ in the definition of the set $\mathcal{X}_{\mathcal{L}}$ and of the category $C_{\mathcal{L}}$ in 8.2 we obtain a set $\mathcal{X}_{\mathbf{Q}_{l}}^{\prime}$ and a category $C_{\overline{\mathbf{Q}}_{l}}^{\prime}$. Note that the objects of $C_{\overline{\mathbf{Q}}_{l}}^{\prime}$ are the elements of $\mathcal{X}_{\overline{\mathbf{Q}}_{l}}^{\prime}$; that is, the sequences $\mathbf{S}=\left(S_{1}, S_{2}, \ldots, S_{b}\right)$ in $W_{\mathcal{L}}$. For $\tilde{\mathbf{S}}, \mathbf{S}$ in $\mathcal{X}_{\overline{\mathbf{Q}}_{l}}^{\prime}$ we denote by $V_{\tilde{\mathbf{S}} \mathbf{S}}^{\prime}$ the vector space of morphisms from $\tilde{\mathbf{S}}$ to $\mathbf{S}$ in $C_{\overline{\mathbf{Q}}_{l}}^{\prime}$.

The following is conjecturally a functor $\Phi: C_{\mathcal{L}} \rightarrow C_{\overline{\mathbf{Q}}_{l}}^{\prime}$. To an object $\mathbf{s}$ of $C_{\mathcal{L}}, \Phi$ associates the object $\mathbf{S}$ of $C_{\overline{\mathbf{Q}}_{l}}^{\prime}$ defined as in 5.5. Given two objects $\tilde{\mathbf{s}}, \mathbf{s}$ of $C_{\mathcal{L}}$ we set $\tilde{\mathbf{S}}=\Phi(\tilde{\mathbf{s}}), \mathbf{S}=\Phi(\mathbf{s})$ and we define a linear map $\Phi: V_{\tilde{\mathbf{s}}, \mathbf{s}} \rightarrow V_{\tilde{\mathbf{S}}, \mathbf{S}}^{\prime}$ to be the isomorphism which maps the distinguished basis of $V_{\tilde{\mathbf{s}}, \mathbf{s}}$ onto the analogous distinguished basis of $V_{\tilde{\mathbf{S}}, \mathbf{S}}^{\prime}$ according to the bijection $\mathcal{A}(W, \mathbf{c}, \mathcal{L}, \mathbf{s}, \tilde{\mathbf{s}}) \stackrel{\sim}{\rightarrow} \mathcal{A}\left(W_{\mathcal{L}}, \omega \mathbf{c}, \overline{\mathbf{Q}}_{l}, \mathbf{S}, \tilde{\mathbf{S}}\right)$ described in 5.10. (As pointed out in 8.1, the set $\mathfrak{F}$ which appears in 5.10 is in our case equal to $\{1\}$.) We expect that $\Phi$ is a functor and that moreover it is an equivalence of categories. 


\section{INDEX OF NOTATION}

1.1. $\mathbf{k}, G, \mathcal{B}, B, T, N(T), W, \operatorname{pos}, l(w), \mathbf{I}$

1.2. $\leq,{ }^{J} W, W^{J^{\prime}},{ }^{J} W^{J^{\prime}}, w_{\mathbf{I}}$

1.3. $\mathcal{P}, U_{P}, U, \mathcal{P}_{J}, L_{J}, P_{B^{\prime}, J}, P^{Q}, k(g)$

1.4, $R, \check{R}, U_{\alpha}, R^{+}, R^{-}, \alpha_{s}$

1.5. $x_{s}(), y_{s}(), \dot{w},[\mathbf{w}],[\mathbf{w}]^{\bullet}$

1.6. $\mathcal{D}(X), \mathfrak{D}(K), \mathcal{M}(X),{ }^{p} H^{\cdot}(K), f^{\star}(A), \mathcal{M}_{\Gamma}(Y), \mathcal{F}^{\sharp}, K \dashv_{\Gamma} K^{\prime}, E_{X}$

1.7. $\mathcal{S}(T)$

1.10. $R_{\mathcal{L}}, R_{\mathcal{L}}^{+}, W_{\mathcal{L}}, \mathbf{I}_{\mathcal{L}}, \check{R}_{\mathcal{L}}$

2.4. $\mathcal{I}_{\mathbf{s}}, \mathcal{L}, F: G \rightarrow G, \Gamma, \mathcal{S}(T)^{w F}, F_{0}: T \rightarrow T, \mathbf{c}$

2.5. $Z^{\mathbf{w}}, \mathfrak{T}, \mathcal{B}_{w}, \mathcal{L}_{\mathbf{w}}, \mathcal{L}_{w}$

2.6. $\bar{Z}^{\mathrm{s}}, \mathcal{Z}^{\mathrm{s}}$

2.11. $\overline{\mathcal{L}}$

3.2. $\Pi^{\mathbf{w}}, \Upsilon^{\mathbf{w}}, \bar{\Upsilon}^{\mathbf{s}}$

3.7. $\mathbb{S}^{\prime}\left(\mathcal{P}_{J}\right)$

$4.1 \mathcal{P}_{J}^{\mathrm{t}}$

4.2. $\mathcal{T}^{\prime}(J, \mathbf{c}), \vartheta$

4.3. $\tilde{\mathcal{P}}_{J}^{\mathrm{t}}$

4.4. $\underline{M}, \mathbb{S}\left(\mathcal{P}_{J}^{\mathrm{t}}\right), \mathbb{S}\left(\mathcal{P}_{J}\right)$

5.6. $W_{\mathcal{L}}^{\prime}$

5.9. $\mathcal{A}(W, c, \mathcal{L}, \mathbf{s}, \tilde{\mathbf{s}})$

$6.3 \mathcal{T}$

6.9. $N_{\mathbf{a}}$

7.1. $X$

\section{REFERENCES}

[BE] R. Bédard, On the Brauer liftings for modular representations, J. Algebra 93 (1985), 332-353. MR.786758 (87a:20041)

[BBD] A. Beilinson, J. Bernstein and P. Deligne, Faisceaux pervers, Astérisque 100 (1982), 5-171. MR751966 (86g:32015)

[Bo] A. Borel, Linear algebraic groups, Benjamin, New York, Amsterdam, 1969. MR0251042 (40:4273)

[DL] P. Deligne and G. Lusztig, Representations of reductive groups over finite fields, Ann. Math. 103 (1976), 103-161. MR0393266 (52:14076)

[L1] G. Lusztig, Characters of reductive groups over a finite field, Ann. Math. Studies 107, Princeton U. Press, 1984. MR742472 (86j:20038)

[L2] G. Lusztig, Character sheaves I, Adv. Math. 56 (1985), 193-237; II, Adv. Math. 57 (1985), 226-265. MR792706 (87b:20055) MR0806210(87m:20118a)

[L3] G. Lusztig, Green functions and character sheaves, Ann. Math. 131 (1990), 355-408. MR 1043271 (91c:20054)

[L4] G. Lusztig, Homology bases arising from reductive groups over a finite field, in "Algebraic groups and their representations", ed. R. W. Carter et al., Kluwer Acad. Publ., 1998, pp. 53-72. MR1670764 (2000b:20054)

[L5] G. Lusztig, Parabolic character sheaves I, II, Moscow Math. J. 4 (2004), 153-179, 869-896. MR2074987 (2006d:20091a) MR2124170 (2006d:20091b)

[L6] G. Lusztig, Character sheaves on disconnected groups, VI, Represent. Theory (electronic) 8 (2004), 377-413. MR 2084488 (2005h:20112)

Department of Mathematics, Massachusetts Institute of Technology, Cambridge, MASSACHUSETtS 02139 